GIOVANNI AIOSA DO AMARAL

\title{
Analytical Assessment of the Mooring System Stiffness
}

São Paulo 



\title{
GIOVANNI AIOSA DO AMARAL
}

\author{
Analytical Assessment of the \\ Mooring System Stiffness
}

Corrected Version

Dissertation presented to Escola Politécnica da Universidade de São Paulo to obtain the degree of Master of Science.

São Paulo 



\title{
GIOVANNI AIOSA DO AMARAL
}

\author{
Analytical Assessment of the \\ Mooring System Stiffness
}

Corrected Version

Dissertation presented to Escola Politécnica da Universidade de São Paulo to obtain the degree of Master of Science.

Concentration Area Structural Engineering.

Advisor: Prof. Dr. Guilherme Rosa Franzini

São Paulo

2020 
Autorizo a reprodução e divulgação total ou parcial deste trabalho, por qualquer meio convencional ou eletrônico, para fins de estudo e pesquisa, desde que citada a fonte.

Este exemplar foi revisado e corrigido em relação à versão original, sob responsabilidade única do autor e com a anuência de seu orientador.

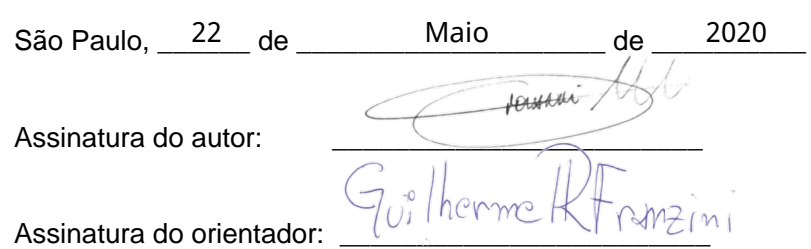

\section{Catalogação-na-publicação}

Amaral, Giovanni Aiosa do

Analytical Assessment of the Mooring System Stiffnes / G. A. Amaral -versão corr. -- São Paulo, 2020.

$151 \mathrm{p}$.

Dissertação (Mestrado) - Escola Politécnica da Universidade de São Paulo. Departamento de Engenharia de Estruturas e Geotécnica.

1.Matriz de Rigidez 2.Formulação Analítica 3.Cabos de Amarração 4.Amarração 5.Ancoragem I.Universidade de São Paulo. Escola Politécnica. Departamento de Engenharia de Estruturas e Geotécnica II.t. 
This text is dedicated to the two women of my life. Sandra Aiosa, my mother, who always pushed me to continue my studies and Vitória de Sousa Chao, my eternal companion, for all the patience through the years. I am grateful for their love. 



\section{Acknowledgements}

This text is obviously not an one-man achievement. It would not be possible to accomplish this master dissertation without the effort of many other people who directly and indirectly participated on this work. Here, some of them are personally thanked.

First of all, I would like to thank Prof. Dr. Guilherme Rosa Franzini, my supervisor. This brilliant man, besides being an amazing supervisor, is an inspiration as a researcher, professor and person. This work would not be possible without his advices and supervision. It has been an honor being your padawan since my early days as a researcher, Master!

I thank also Prof. Dr. Celso Pupo Pesce, for all the advices (personal and professional) and the motivation for the present research. I am grateful for his efforts for the creation and maintenance of LMO.

Thank you also to Prof. Dr. Alfredo Gay Neto, Prof. Dr. Alexandre Simos and Prof. Dr. Carlos Mazzilli for the advices and contributions through the last years.

To Prof. Dr. Renato Orsino, Prof. Dr. Clovis Martins, Prof. Dr. Rodrigo Provasi and Dr. Fernanda Takafuji for being references for me at the laboratory space, acting as counselors and friends.

I thank Eng. Guilherme Vernizzi and Eng. Lucas Carmo for all the advices and patience and Vitor Schwenck for the help with the beautiful images presented in this text. In addition, I thank very much my pupil Guilherme Martins for his great work with the numerical validation of some results from this dissertation.

Of course, I thank very much my laboratory fellows: Leticia Madi, Igor Mancilla, Renato Finotelli, Fernando Toni, Wagner Defensor, Tatiana Ueno, Heloisa Beraldo, Daniela Kawagutti, Michel Freitas, João Cerqueira, Mohammad Moharrami, Guilherme Morita and Pauline Kölb. Our team is ready for all the future challenges!

A great thank to Larissa Costa and Edite Almeida for taking care of all these LMO grown children, giving us an excellent work environment.

Finally, I am grateful to FUSP/ONRG and FUSP/Petrobras for my Master scholarship in the context of the project ONRG nr. N62909-16-1-2066 and Petrobras nr. 2018/00378-7, respectively. This master dissertation is also part of the activities developed in a bilateral research project sponsored by CAPES and JSPS, grant 88881.191007/2018-01. I also thank the Programa de Pós-Graduação em Engenharia Civil from Escola Politécnica of the University of São Paulo. 

"Passion is the fuel for success."

Kobe Bean Bryant (1978 - 2020) 



\section{Abstract}

AMARAL, G. A. Analytical Assessment of the Mooring System Stiffness. 2020. 153p. Dissertation (Master of Sciences) - Escola Politécnica, University of São Paulo, São Paulo, 2020.

The definition of the mooring systems is one of the most important stages on the design any offshore unit. Some effects associated with it, on the responses of the floating body, are still under investigation. In this context, the full nonlinear high-order hierarchical modeling and the numerical integration of the resulting equations of motion might not be the most cost effective approach for the evaluation of those effects during the early design process. Thus, an expedite analytical formulation to assess the mooring system stiffness, a tool that could help the initial design and analysis. Using classic approaches from Analytical Mechanics, the nonlinear generalized restoring forces associated with the mooring acting on the vessel due to the mooring lines are formulated. The six-degree-of-freedom (DoF) problem is herein addressed. The stiffness matrix is obtained from the linearization of the generalized forces around a generic position. Mooring line characteristic tension function is an input of the method. The closed formulation does not requires a specific line model, although the formulation for a multi-segment mooring line is also derived. The methodology is applied taking the OC4-DeepCwind Floating Wind Turbine semi-submersible platform as a case study. Two Spread Mooring Systems arrangements are studied, in order to demonstrate the use of the presented formulation as a design tool. The calculated mooring system stiffness matrix, evaluated at the trivial equilibrium position, exhibits good agreement with numerical results found in the literature by high hierarchy models. Additionally, the stiffness coefficients are evaluated for other positions than the trivial equilibrium one in the form of colored maps. The natural periods of the motions on the horizontal plane are also mapped. These maps help to understand the effects of the static vessel mean position on the mooring system stiffness and, consequently, on the natural periods associated with the motions on the horizontal plane. Considering the original OC4 mooring system, the effects of the mooring line pre-tensioning are also investigated. Some conclusions on the axial stiffness of catenary cables are also made. The main contributions of the present master dissertation are: (i) the stiffness matrix analytical closed formulation and (ii) the use of colored maps to evaluate the stiffness and the natural periods as functions of the mean offset position. The present master dissertation brings then an innovative closed-form formulation with important practical applications.

Keywords: Stiffness matrix, analytical formulation, mooring cables, mooring, anchoring. 



\section{Resumo}

AMARAL, G. A. Avaliação Analítica da Rigidez de Sistemas de Amarração. 2020. 153p. Dissertação (Mestrado) - Escola Politécnica, Universidade de São Paulo, São Paulo, 2020 .

O projeto do sistema de amarração é uma das etapas mais importantes para qualquer unidade offshore e alguns efeitos associados a ele nas respostas do corpo flutuante ainda estão sob investigação. Nesse contexto, a modelagem não linear de alta hierarquia e a integração numérica das equações de movimento resultantes pode não ser a abordagem mais eficiente computacionalmente para a investigação desses efeitos durante o projeto. Assim, é proposta uma formulação analítica expedita para determinar a matriz de rigidez devido ao sistema de amarração, ferramenta que pode auxiliar o projeto e a análise dos sistemas, em particular em suas fases iniciais. Usando abordagens clássicas da mecânica analítica, as forças generalizadas associadas ao sistema de amarração são formuladas. $\mathrm{O}$ problema de seis graus de liberdade (GL) é abordado. A matriz de rigidez é obtida ao se linearizar as forças generalizadas decorrentes do sistema de amarração em torno de uma posição genérica. Embora a formulação fechada não necessite de nenhum modelo específico de linha de amarração, ainda assim a formulação de uma linha com múltiplos segmentos é apresentada. A metodologia é aplicada usando a plataforma semissubmerssível da turbina eólica flutuante OC4-DeepCwind como um estudo de caso. Dois sistemas de amarração do tipo Spread Mooring System são estudados, demonstrando o uso da formulação apresentada como uma ferramenta para projeto. A matriz de rigidez associada ao sistema de amarração avaliada na posição trivial de equilíbrio, calculada analiticamente utilizando a metodologia proposta, apresenta aderência com aquelas obtidas na literatura via métodos numéricos de alta hierarquia. Complementarmente, os coeficientes de rigidez são avaliados para outras posições distintas daquela de equilíbrio trivial, com os resultados apresentados na forma de mapas de cores. Os períodos naturais para os modos no plano horizontal também são mapeados. Esses mapas auxiliam no entendimento dos efeitos da posição estática média do corpo flutuante na rigidez do sistema de amarração e períodos naturais. Para o sistema de amarração original da OC4, os efeitos da pré-tração são investigados. Algumas conclusões sobre a rigidez axial em cabos em catenária também são realizadas. As principais contribuições desta dissertação são: (i) a formulação analítica da matriz de rigidez e (ii) o uso de mapas de cor para a avaliação da rigidez e dos períodos naturais em função da posição média. Esta dissertação de mestrado apresenta, portanto, uma formulação fechada com importantes aplicações práticas.

Palavras-chave: Matriz de rigidez, formulação analítica, cabo de amarração, amarração, ancoragem. 



\section{List of Figures}

Figure 1 - Mooring line profile: (a) Catenary laying, (b) Taut-leg, (c) Tension-leg and (d) Intermediate buoy. . . . . . . . . . . . . . . . . . . 29

Figure 2 - Mooring line materials. . . . . . . . . . . . . . . . . . . . . . . . 29

Figure 3 - Mooring systems arrangement: (a) Spread Mooring System (SMS), (b) Internal Turrent Mooring System, (c) External Turrent (box) Mooring System and (d) Single Point Mooring (SPM). . . . . . . . . . 30

Figure 4 - Mooring systems patterns: (a) $4 \times 1$, (b) $4 \times 2$ and (c) $4 \times 5$. . . . . . 31

Figure 5 - Offshore Oil \& Gas Platforms: (a) fixed, (b) compliant, (c) Tension-leg, (d) Spar, (e) Semi-submersible and (f) Floating Production Storage and Offloading (FPSO) . . . . . . . . . . . . . . . . 32

Figure 6 - Offshore Wind Turbines Platforms: (a) Gravity based, (b) Monopile, (c) Tripile, (d) Tripod, (e) Jacket, (f) Spar, (g) Tension-leg and (h) Semi-submersible. . . . . . . . . . . . . . . . . . 33

Figure 7 - Typical mooring design and analysis workflow. . . . . . . . . . 36

Figure 8 - Relation between anchor radius and water depth, based on the number of existing facilities. . . . . . . . . . . . . . 37

Figure 9 - Quasi-static and dynamic mooring analyses. . . . . . . . . . 40

Figure 10 - Overall design procedure. . . . . . . . . . . . . . . 41

Figure 11 - Nonlinear time domain response. . . . . . . . . . . . . . . . . . 44

Figure 12 - Catastrophe set. . . . . . . . . . . . . . . . 44

Figure 13 - Flow-chart of mooring system design. . . . . . . . . . . . . . 46

Figure 14 - Sketch of a four line SMS. . . . . . . . . . . . . . . . 48

Figure 15 - Geometry of hybrid line. . . . . . . . . . . . . . . . . 48

Figure 16 - Geometry of buoy-supported line. . . . . . . . . . . . . . . . . 49

Figure 17 - Three segment mooring line with a clump weight. . . . . . . . . . 49

Figure 18 - Generic geometry of a multi-segment mooring line. . . . . . . . . . 51

Figure 19 - Sketch of the moored floating body. . . . . . . . . . . . 53

Figure 20 - Small horizontal displacements and rotations. . . . . . . . . . . 53

Figure 21 - Vertical plane view of a SMS. . . . . . . . . . . . . . . 54

Figure 22 - Six-DoF moored system. . . . . . . . . . . . . . . 55

Figure 23 - Sketch of a generic moored buoy as a generic position. . . . . . . . 56

Figure 24 - Sketch of a generic floating body, moored by N mooring lines at: the equilibrium position of the autonomous system (dashed) and a generic displaced position (full lines). . . . . . . . . . . . . . . 60

Figure 25 - Translational displacement between $G \xi \eta \zeta$ and $O x y z$. . . . . . . . . 60

Figure 26 - Definition of the Euler Angles. . . . . . . . . . . . . . . . . 61 
Figure 27 - Generic floating body, moored by N mooring lines at a generic position. 61

Figure 28 - Mooring line planes and directional unit vectors. . . . . . . . . . . . 63

Figure 29 - i-th mooring line tension at the fairlead. . . . . . . . . . . . . . 64

Figure 30 - Multi-segment mooring line profile. . . . . . . . . . . . . . 73

Figure 31 - Forces acting on an infinitesimal mooring element. . . . . . . . . . . . . 74

Figure 32 - Free-body diagram for $s_{1}$ and $s_{j} \ldots \ldots \ldots \ldots$

Figure 33 - The OC4-DeepCwind floating wind system design. . . . . . . . . . . . 80

Figure 34 - The OC4-DeepCwind floating wind added masses and moment of inertia for the planar motions. Determined with WAMITß. . . . . . . . . . 81

Figure 35 - Step-by-step procedure for the mooring system stiffness calculation. . . 81

Figure 36 - Top view of the OC4-DeepCwind floating wind original mooring system. 82

Figure 37 - One-segment all-chain mooring line profile of the OC4-DeepCwind floating wind original mooring system. . . . . . . . . . . . . . . 83

Figure 38 - The OC4-DeepCwind chain mooring line characteristic curves. Unities: $\mathrm{N}$ and $\mathrm{m} . \ldots \ldots \ldots \ldots$. . . . . . . . . . . . . . 86

Figure 39 - Comparison between the characteristic curves for the extensible and the non-extensible catenary mooring models. Unities: $\mathrm{N}$ and $\mathrm{m} . \quad$. . . . 87

Figure 40 - The OC4-DeepCwind modes of oscillation for the trivial position, corresponding to natural periods $T_{j}, j=1,2,3 \ldots \ldots 88$

Figure 41 - Partition $\mathbb{K}_{T T}$ of the stiffness matrix as function of the offset. Unities:

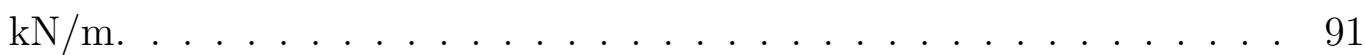

Figure 42 - Partitions $\mathbb{K}_{T R}$ and $\mathbb{K}_{R T}$ of the stiffness matrix as function of the offset. Unities: $\mathrm{kN} / \mathrm{rad}\left(\mathbb{K}_{T R}\right)$ or $\mathrm{kNm} / \mathrm{m}\left(\mathbb{K}_{R T}\right)$. . . . . . . . . . . . . . 92

Figure 43 - Partition $\mathbb{K}_{R R}$ of the stiffness matrix as function of the offset. Unities: $\mathrm{kNm} / \mathrm{rad}$. . . . . . . . . . . . . . . . 93

Figure 44 - The OC4-DeepCwind natural periods as function of the offset. Unity: s. 94

Figure 45 - The OC4-DeepCwind natural periods as function of the offset: preponderant directions. Unity: s. . . . . . . . . . . . . . . . . . . . . . . 94

Figure 46 - The OC4-DeepCwind modes of oscillation for the trivial and shifted equilibrium positions, corresponding to natural periods $T_{j}, j=1,2,3$. . 95

Figure 47 - Partition $\mathbb{K}_{T T}$ of the stiffness matrix as function of the offset. Heading $=\bar{\psi}=5^{\circ}$. Unities: $\mathrm{kN} / \mathrm{m}$. . . . . . . . . . . . . . 97

Figure 48 - Partitions $\mathbb{K}_{T R}$ and $\mathbb{K}_{R T}$ of the stiffness matrix as function of the offset. Heading $=\bar{\psi}=5^{\circ}$. Unities: $\mathrm{kN} / \mathrm{rad}\left(\mathbb{K}_{T R}\right)$ or $\mathrm{kNm} / \mathrm{m}\left(\mathbb{K}_{R T}\right) \ldots$. . . . 98

Figure 49 - Partition $\mathbb{K}_{R R}$ of the stiffness matrix as function of the offset. Heading $=\bar{\psi}=5^{\circ}$. Unities: $\mathrm{kNm} / \mathrm{rad}$.

Figure 50 - Partition $\mathbb{K}_{T T}$ of the stiffness matrix as function of the offset. Low pre-tensioning case. Pre-tensioning ratio: $f^{*}=0.9$. Unities: $\mathrm{kN} / \mathrm{m} . \quad$. . 101 
Figure 51 - Partitions $\mathbb{K}_{T R}$ and $\mathbb{K}_{R T}$ of the stiffness matrix as function of the offset. Low pre-tensioning case. Pre-tensioning ratio: $f^{*}=0.9$. Unities: $\mathrm{kN} / \mathrm{rad}$

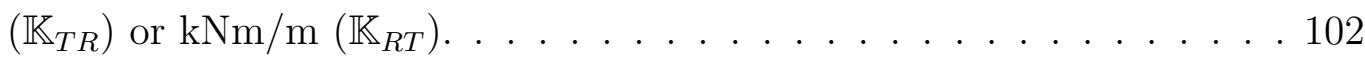

Figure 52 - Partition $\mathbb{K}_{R R}$ of the stiffness matrix as function of the offset. Low pre-tensioning case. Pre-tensioning ratio: $f^{*}=0.9$. Unities: $\mathrm{kNm} / \mathrm{rad}$. . 103

Figure 53 - Partition $\mathbb{K}_{T T}$ of the stiffness matrix as function of the offset. Design pre-tensioning case. Pre-tensioning ratio: $f^{*}=1.0$. Unities: $\mathrm{kN} / \mathrm{m}$. . . 104

Figure 54 - Partitions $\mathbb{K}_{T R}$ and $\mathbb{K}_{R T}$ of the stiffness matrix as function of the offset. Design pre-tensioning case. Pre-tensioning ratio: $f^{*}=1.0$. Unities: $\mathrm{kN} / \mathrm{rad}\left(\mathbb{K}_{T R}\right)$ or $\mathrm{kNm} / \mathrm{m}\left(\mathbb{K}_{R T}\right)$. . . . . . . . . . . . . 105

Figure 55 - Partition $\mathbb{K}_{R R}$ of the stiffness matrix as function of the offset. Design pre-tensioning case. Pre-tensioning ratio: $f^{*}=1$.0. Unities: $\mathrm{kNm} / \mathrm{rad}$. . 106

Figure 56 - Partition $\mathbb{K}_{T T}$ of the stiffness matrix as function of the offset. High pre-tensioning case. Pre-tensioning ratio: $f^{*}=1.1$. Unities: $\mathrm{kN} / \mathrm{m} . \quad$. . 107

Figure 57 - Partitions $\mathbb{K}_{T R}$ and $\mathbb{K}_{R T}$ of the stiffness matrix as function of the offset. High pre-tensioning case. Pre-tensioning ratio: $f^{*}=1.1$. Unities: $\mathrm{kN} / \mathrm{rad}$ $\left(\mathbb{K}_{T R}\right)$ or $\mathrm{kNm} / \mathrm{m}\left(\mathbb{K}_{R T}\right) \ldots \ldots \ldots$. . . . . . . . . . . . 108

Figure 58 - Partition $\mathbb{K}_{R R}$ of the stiffness matrix as function of the offset. High pre-tensioning case. Pre-tensioning ratio: $f^{*}=1.1$. Unities: $\mathrm{kNm} / \mathrm{rad}$. . 109

Figure 59 - The OC4-DeepCwind natural periods as function of the offset and the pre-tensioning. Unities: s. . . . . . . . . . . . . . . 110

Figure 60 - The OC4-DeepCwind floating wind alternative mooring system: (a) Top view and (b) Chain-wire-chain mooring line profile. . . . . . . . . . . . 111

Figure 61 - Partition $\mathbb{K}_{T T}$ of the stiffness matrix as function of the offset. Alternative mooring system. Unities: kN/m. . . . . . . . . . . . . . . . . . 115

Figure 62 - Partitions $\mathbb{K}_{T R}$ and $\mathbb{K}_{R T}$ of the stiffness matrix as function of the offset. Alternative mooring system. Unities: $\mathrm{kN} / \mathrm{rad}\left(\mathbb{K}_{T R}\right)$ or $\mathrm{kNm} / \mathrm{m}\left(\mathbb{K}_{R T}\right)$.

Figure 63 - Partition $\mathbb{K}_{R R}$ of the stiffness matrix as function of the offset. Alternative mooring system. Unities: kNm/rad . . . . . . . . . . . . . . . . . 117

Figure 64 - The OC4-DeepCwind natural periods as function of the offset. Alternative mooring system. Unities: s. . . . . . . . . . . . . . . . . . . . . 118

Figure 65 - Multi-segment mooring line profile. . . . . . . . . . . . . . . . . 130

Figure 66 - Sketch of the projection of a moored floating body in a generic offset position and heading onto the horizontal plane $\pi$. . . . . . . . . . . . 151 



\section{List of Tables}

Table 1 - Guidance for mooring system profile and composition selection. . . . . . 30

Table 2 - Stiffness matrix models presented at the reviewed literature. . . . . . . 57

Table 3 - Platform structural and hydrodynamic properties. . . . . . . . . . . 80

Table 4 - The OC4-DeepCwind original mooring system parameters. . . . . . . 83

Table 5 - The OC4-DeepCwind original mooring line forces and stiffness. . . . . . 84

Table 6 - Natural periods of oscillation for the trivial position. Unities: s. . . . . . 87

Table 7 - Pre-tensioning cases: total length, mooring line forces and stiffness. . . . 88

Table 8 - Study of the pre-tensioning effect on the natural periods of oscillation for the trivial position. Unity: s. . . . . . . . . . . . . . . . 89

Table 9 - The OC4-DeepCwind alternative mooring system parameters. . . . . . . 112

Table 10 - The OC4-DeepCwind alternative mooring line forces and stiffness. . . . 112

Table 11 - The OC4-DeepCwind natural periods of oscillation for the trivial position: comparison between original and alternative mooring systems. Unities: s. 113 



\section{List of abbreviations and acronyms}

$\begin{array}{ll}\text { DoF } & \text { Degree-of-Freedom } \\ \text { DICAS } & \text { Differentiated Compliance Anchoring Systems } \\ \text { FOWT } & \text { Finite Elements } \\ \text { FPSO } & \text { Floating Prfshore Wind Turbine } \\ \text { MS } & \text { Multi-segment } \\ \text { OC4 } & \text { Offshore Code Comparison Collaboration Continuation } \\ \text { O\&G } & \text { Oil \& Gas } \\ \text { RAO } & \text { Response amplitude operators } \\ \text { SWL } & \text { Sea Water Level } \\ \text { SPM } & \text { Single Point Mooring } \\ \text { SMS } & \text { Spread Mooring Systems } \\ \text { TDP } & \text { Touchdown Point }\end{array}$





\section{List of symbols}

Oxyz inertial reference frame, grounded to earth

$G \xi \eta \zeta \quad$ moving reference frame, fixed to the body

$E_{x} \quad$ vector base defining $O x y z$

$E_{\xi} \quad$ vector base defining $G \xi \eta \zeta$

$\left(\hat{e}_{x}, \hat{e}_{y}, \hat{e}_{z}\right) \quad$ base $E_{x}$ vectors

$\left(\hat{e}_{\xi}, \hat{e}_{\eta}, \hat{e}_{\zeta}\right) \quad$ base $E_{\xi}$ vectors

$\mathbf{r} \quad$ translational displacement of $G \xi \eta \zeta$ with respect to $O x y z$

$\left[\begin{array}{lll}r_{x} & r_{y} & r_{z}\end{array}\right]^{t} \quad$ components of $\mathbf{r}$

$\boldsymbol{\theta} \quad$ Euler's angles

$\left[\begin{array}{lll}\phi & \theta & \psi\end{array}\right]^{t} \quad$ components of $\boldsymbol{\theta}$

q generalized coordinates

$\vec{P}^{(i)} \quad$ fairlead position of the i-th mooring line

$\vec{A}^{(i)} \quad$ anchor position of the i-th mooring line

$h_{f}^{(i)} \quad$ horizontal fairlead-anchor distance of the i-th mooring line

$v_{f}^{(i)} \quad$ vertical fairlead-anchor distance of the i-th mooring line

$\hat{e}_{h}^{(i)} \quad$ horizontal directional vector of the i-th mooring line

$\hat{e}_{v}^{(i)} \quad$ vertical directional vector of the i-th mooring line

$\alpha^{(i)} \quad$ angle between the i-th mooring line and the $O x$ (counterclock wise)

$\vec{T}^{(i)} \quad$ tension at the fairlead for the i-th mooring line

$F_{H}(i) \quad$ horizontal component of $\vec{T}^{(i)}$

$F_{V}(i) \quad$ vertical component of $\vec{T}^{(i)}$

Q generalized restoring forces

$\mathbb{K} \quad$ mooring system stiffness matrix 
$\mathbb{K}_{T T} \quad$ translational-translational coefficients of the i-th mooring line stiffness matrix

$\mathbb{K}_{T R} \quad$ translational-rotational coefficients of the i-th mooring line stiffness matrix

$\mathbb{K}_{R T} \quad$ rotational-translational coefficients of the i-th mooring line stiffness matrix

$\mathbb{K}_{R R} \quad$ rotational-rotational coefficients of the i-th mooring line stiffness matrix 


\section{Contents}

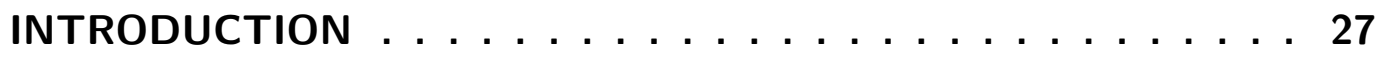

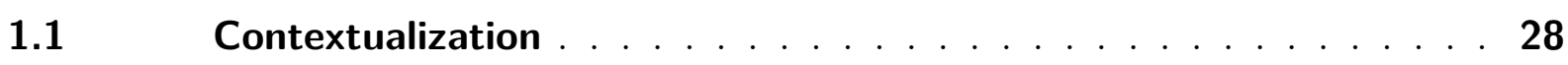

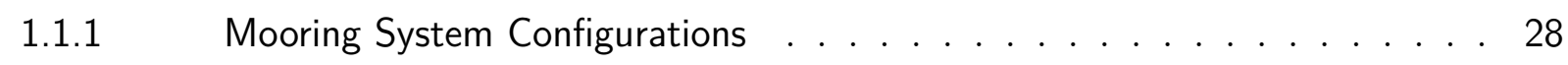

1.1 .2 Types of Offshore Platforms . . . . . . . . . . . . . . 31

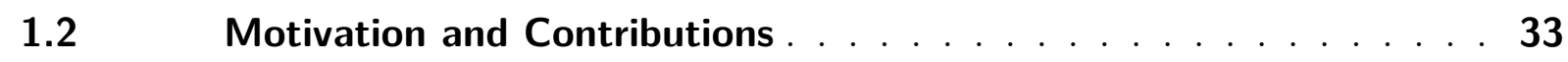

$1.3 \quad$ Text Organization $\ldots \ldots \ldots \ldots \ldots$

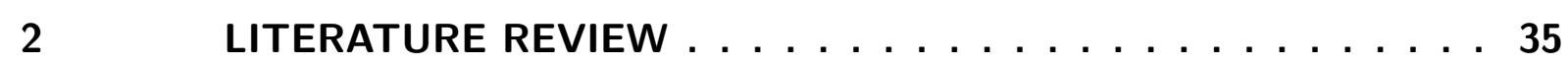

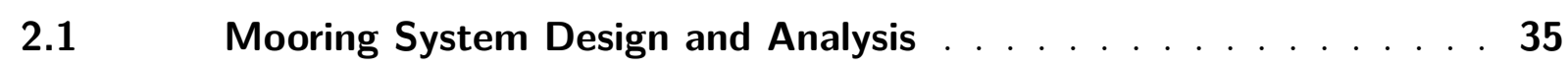

2.2 Mooring System Design Tools . . . . . . . . . . . 41

$2.3 \quad$ Mooring Restoring Forces . . . . . . . . . . . . . . . 46

$2.4 \quad$ Mooring System Stiffness Matrix . . . . . . . . . . . . 51

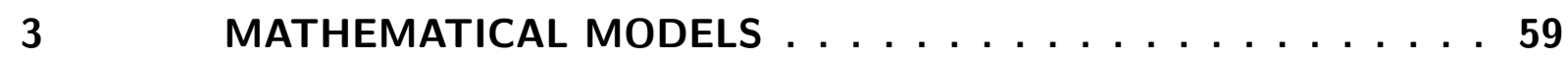

$3.1 \quad$ Geometric Relations of Floating Unit and Mooring Lines . . . . . . 59

$3.2 \quad$ Mooring System Restoring Forces . . . . . . . . . . . . 64

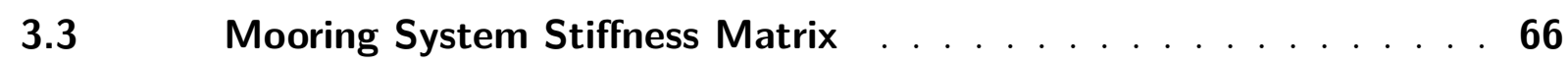

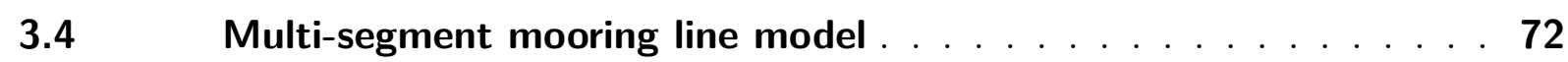

4 ANALYSES OF SPREAD MOORING SYSTEMS $\ldots \ldots \ldots 79$

$4.1 \quad$ All-chain catenary mooring line . . . . . . . . . 82

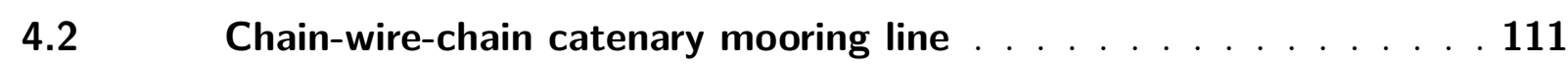

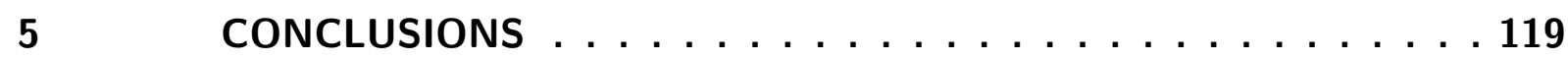

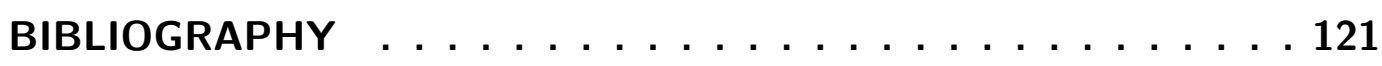

$\begin{array}{ll}\text { APPENDIX } & 125\end{array}$

APPENDIX A-NUMERICAL EVALUATION OF THE MOORING LINES FORCES AND STIFFNESS . . . . . 127

APPENDIX B - MULTI-SEGMENT MOORING LINE STATIC CON-

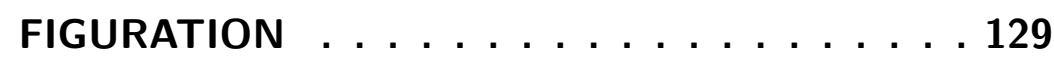


APPENDIX C - THE LOCAL MOORING LINE GENERALIZED DIRECTIONS AND THEIR PROJECTIONS ONTO HORIZONTAL AND VERTICAL PLANES . . . 131

APPENDIX D - INTERMEDIATE DEVELOPMENTS FOR THE MOORING SYSTEM STIFFNESS COEFFICIENTS133

APPENDIX E - ALGEBRAIC MANIPULATION OF THE STIFFNESS COEFFICIENTS . . . . . . . . . 143

APPENDIX F - STIFFNESS MATRIX FOR THE PLANAR PROB-

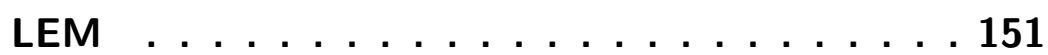




\section{Introduction}

The seek for different and clean sources of energy is one of the major challenges of the 21st century. In this context, oceans can be seen as important and still under-explored scenarios for renewable energy. Even though the offshore Oil \& Gas (O\&G) industry is one of the most competitive players in the world, many other possibilities of offshore energy can be pointed out such as wind, wave and sea current energies.

Some of these alternative sources of energy are associated with structures installed in intermediate and deep waters. This may lead to the need of mooring systems to provide static positioning as well as to guarantee that the dynamic responses do not exceed certain thresholds. The present master dissertation addresses this problem and aims at contributing with the analysis and design of mooring systems.

The design of a mooring system is of crucial importance for any offshore floating project. Not only operational requirements, but also financial demands impact the whole process. A mooring system design has to be able to keep static and dynamic responses of the structure under determined values and also be cost-efficient. It is important to emphasize that this efficiency is even more important for offshore energy other than the O\&G industry.

Additionally, designing a mooring system involves numerous parameters: number, orientation, pre-tensioning and properties of mooring lines, geometric arrangement of the system, among others. The lack of basic understanding of the effects of these parameters normally leads to a high number of nonlinear system simulations. Consequently, the project cost increases.

Offshore renewable energy is highly cost-sensitive. On the one hand, Any overdesign of the mooring system could determine the inappropriateness of offshore energy projects. On the other hand, a complex modeling of the whole problem including the mooring system can be expensive from a computational point-of-view, which also leads to a project cost increase. In this scenario, a good understanding of the effects of mooring system parameters in both static and dynamic responses of the floating units is of crucial importance for the success of a project. Motivated by the context of offshore renewable energy ${ }^{1}$, this masters dissertation proposes a six-degree-of-freedom (DoF) closed-form analytical formulation to assess mooring system stiffness. The herein proposed formulation allows not only to evaluate the matrix for a specific position faster than using numerical static offset analysis, but also to easily map the stiffness coefficients for different offset positions

$\overline{1}$ Even though the motivation is associated with renewable energy from the ocean, the methodology herein developed can be applied for O\&G activities as well. 
and parameters configurations. The stiffness matrix for the trivial equilibrium position analytically calculated using this formulation shows an excellent agreement with numerical results found in literature, as can be seen in Chapter 4 .

The present chapter brings an initial overview on essential topics that contextualize this master dissertation and clarify the motivation and contributions of this work. Finally, the contents of following chapters are presented.

\subsection{Contextualization}

This section presents a brief introduction to important topics that are addressed in this work. In order to better situate the reader, it brings forward an outlook on the different configurations of mooring system and types of floating units nowadays employed.

\subsubsection{Mooring System Configurations}

During the project of a mooring system, the designer has to specify numerous parameters of mooring lines including the number of lines and their materials as well as the desired static configuration. It must also be specified aspects related to the system such as position of anchors and fairleads (i.e. the points of the vessel in which the mooring lines will be attached), among others. The aim of this subsection is to present part of these important parameters, helping the reader to understand the subject's complexity.

It is possible to classify the mooring system by the static profile configuration of its lines: catenary, taut-leg, tension-leg and other variations. Figure 1 brings up four classic configurations. The choice of a particular configuration is based on the water depth, the anchorage radius and also on the vessel offset limit. It is important for future discussions to notice that some of this configurations have a known analytical closed formulations, relating water depth and anchorage radius with line tension - hereafter named as force-displacement relation - while others do not.

It is also possible to classify the mooring system considering the line composition and material. Three possibilities are most commonly found, namely, synthetic ropes, steel wire ropes and steel chains. Different types of material for the synthetic ropes can be used including nylon, polyester, polyethylene, polypropylene. It is also possible to have mixed lines - mainly when the configuration is a semi-taut or intermediate buoy in deep waters. Figure 2 presents examples of mooring lines composed of these materials. The choice is normally based on weight, usage and mobility of the floating unit, but also on the total line length which is, in turn, function of the anchorage radius and water depth.

Wang, Er and Iu (2019) brings a guide for selecting the proper mooring profile and composition depending on the water depth $d$, as presented in Table 1. 
Figure 1 - Mooring line profile: (a) Catenary laying, (b) Taut-leg, (c) Tension-leg and (d) Intermediate buoy.

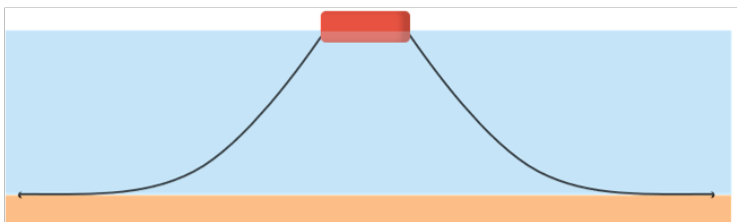

(a)

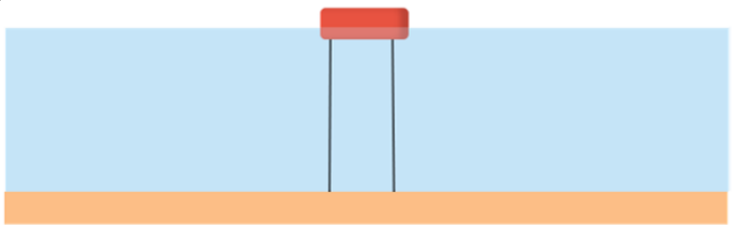

(c)

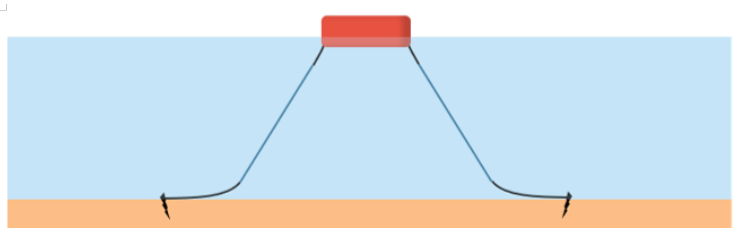

(b)

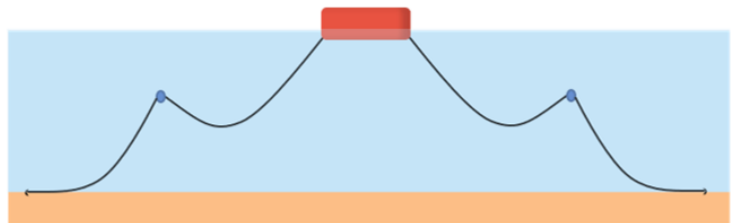

(d)

Source: The Author

Figure 2 - Mooring line materials.

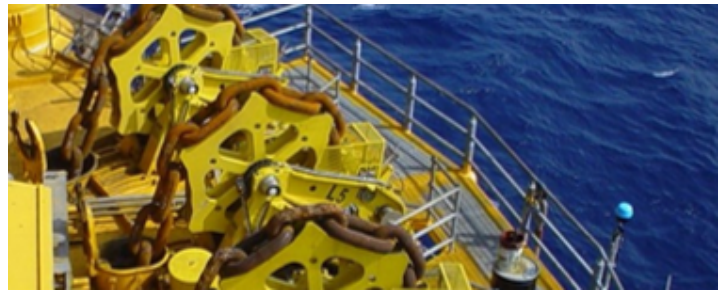

(a) Chain. Source: MacGregor (2017)

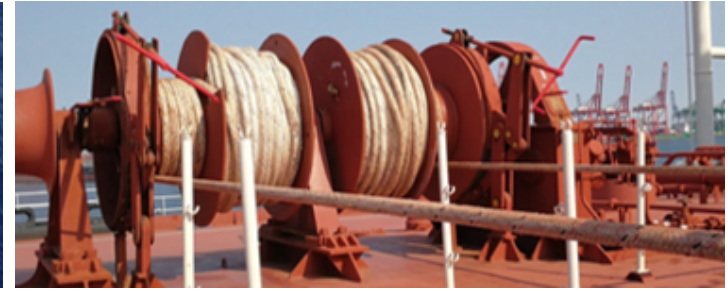

(b) Synthetic rope. Source: Dyneema (2018)

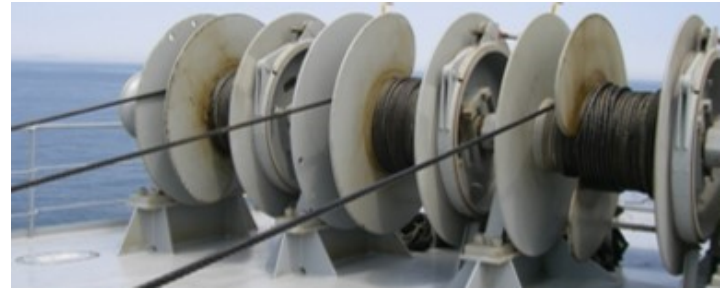

(c) Steel wire rope. Source: SaemaH (2019)

Finally, another possible classification is related to system mooring lines arrangement, i.e. the position of the fairleads. In this context, the $\mathrm{O} \& \mathrm{G}$ industry presents more possibilities when compared to renewable energy sectors, at least at this moment. Figure 3 shows our classical mooring line arrangements used on the O\&G scenario. In turn, floating offshore wind turbines (FOWTs) are mainly characterized by spread mooring systems (SMS), although new concepts are being studied throughtout the last years. The choice of the arrangement can be based on the vessel characteristics and also on the predictability of sea current, wind and wave incidence angles.

Following Figure 3, it is possible to define the difference between the system arrangements: 
Table 1 - Guidance for mooring system profile and composition selection.

\begin{tabular}{ccc}
\hline \hline Water depth & Mooring line profile & Composition \\
\hline$d<500 m$ & Catenary & All-chain \\
$500<d<1000 m$ & Catenary or Semi-taut & All-chain or Chain-wire-chain \\
$1000<d<2000 m$ & Taut-leg or Semi-taut & Chain-wire-chain or Chain-rope-chain \\
$d>2000 m$ & Taut-leg or Semi-taut & Chain-rope-chain \\
\hline \hline
\end{tabular}

Source: Adapted from Wang, Er and Iu (2019)

Figure 3 - Mooring systems arrangement: (a) Spread Mooring System (SMS), (b) Internal Turrent Mooring System, (c) External Turrent (box) Mooring System and (d) Single Point Mooring (SPM).

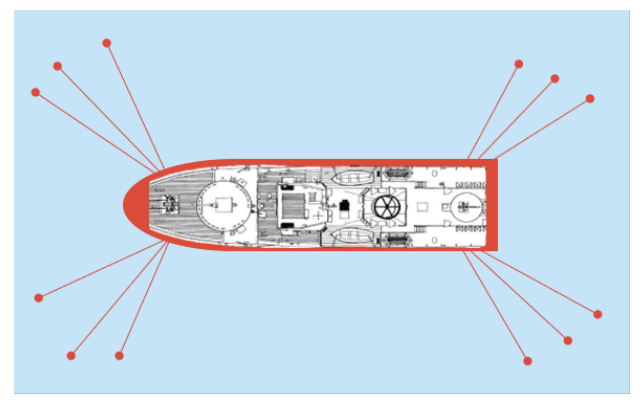

(a)

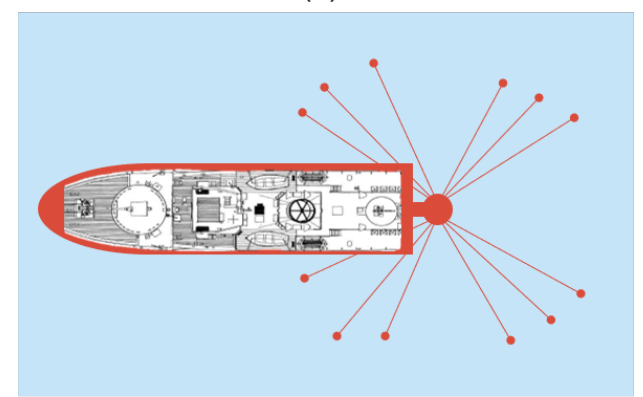

(c)

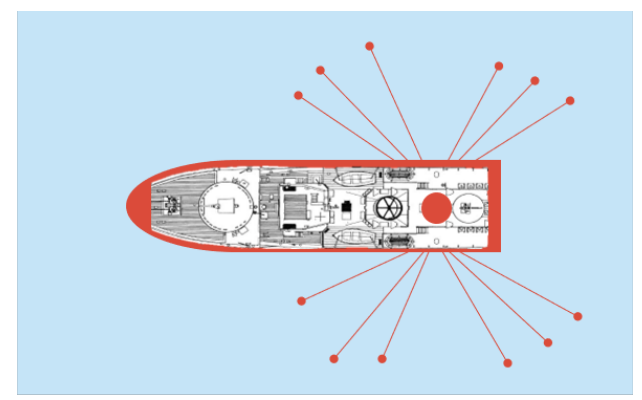

(b)

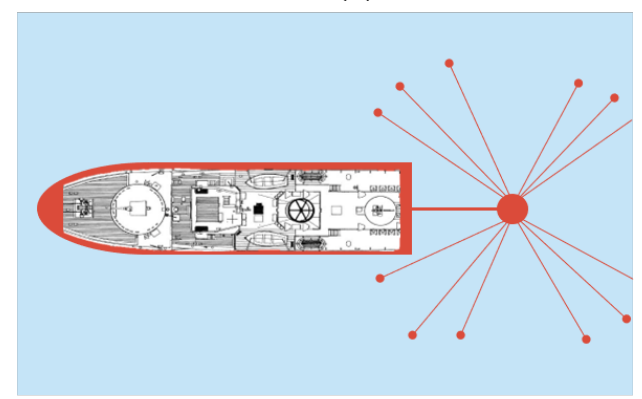

(d)

Source: The Author

- Spread Mooring System (SMS): The fairleads are located along the vessel and vessel rotations are restricted. A particular type of SMS is the differentiated compliance anchoring systems (DICAS), where the stiffness at the bow and stern of the ship are different (Kaster et al., 1997).

- Internal Turret Mooring System: The mooring lines are attached to an internal tower named turret. This tower creates a pivot point for the rotation of the vessel.

- External Turret (Bow) Mooring System: the mooring lines are also attached to a tower, but externally placed and connected to the vessel. Again, the tower creates a pivot point around which the vessel can rotate. 
- Single Point Mooring (SPM): the mooring line are attached to an external buoy. In its turn, the buoy is connected to the vessel through another (and stiffer) mooring line.

Considering SMS, it is also possible to classify the system with respect to mooring patterns. Mooring lines may be all equally spread or grouped in number. In the latter case, it is necessary to define the number of groups and lines, besides the spread angle between them. Figure 4 shows three possibilities for a SMS of a semisubmersible. The $4 \times 1$ pattern normally is not a good choice due to the possibility of a one-line fail, although it is normally used for renewable energy projects. Mooring systems with few lines, such as $4 \times 2$, need greater safety factors for the damaged condition. The increase of the number of lines (and consequently, the redundancy) demands lower safety factors, as the impact of loosing one line is much lower.

Figure 4 - Mooring systems patterns: (a) $4 \times 1$, (b) $4 \times 2$ and (c) $4 \times 5$.

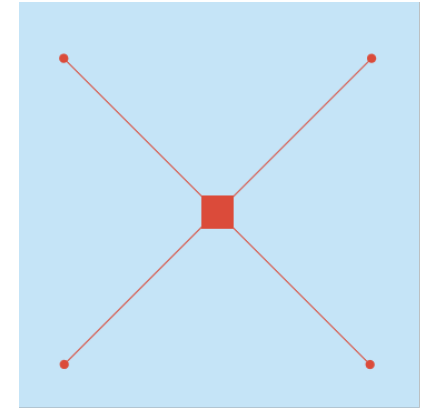

(a)

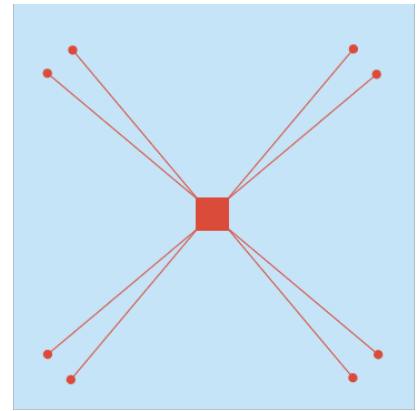

(b)

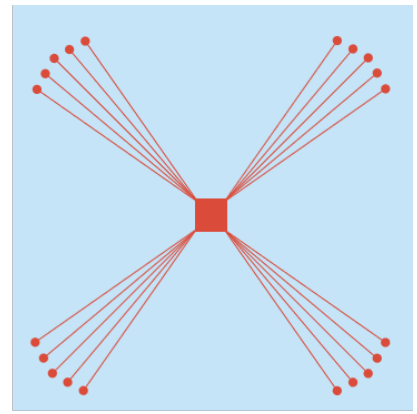

(c)

Source: The Author

\subsubsection{Types of Offshore Platforms}

In the context of ocean energies exploration, platforms play an important role. Indeed, since the initial fixed platforms of the O\&G industry to the new, modern, prototypes of FOWTs, the concept of different types of platforms was and still is a hot-topic of research. In 2016, more than 9500 offshore platforms were installed worldwide (Dhanak and Xiros, 2016) with the corresponding water depths varying from few meters to 3 kilometers. These large structures are responsible for the production of more than 100 million barrels of oil per day, besides other new sources of offshore energy. Herein, some of the most important concepts are presented in order to offer to the reader a clear overview on the theme.

Generally speaking, the offshore platforms can be divided into two main groups: the fixed and compliant platforms, and the floating platforms. The former group is directly attached to the seabed and its usage is defined by a maximum sea depth. The latter is 
used for greater sea depths and its stationkeeping is commonly due to mooring systems. Figures 5 and 6 depict the most common types of offshore platforms for O\&G exploration and Wind Turbines, respectively.

Figure 5 - Offshore Oil \& Gas Platforms: (a) fixed, (b) compliant, (c) Tension-leg, (d) Spar, (e) Semi-submersible and (f) Floating Production Storage and Offloading (FPSO).
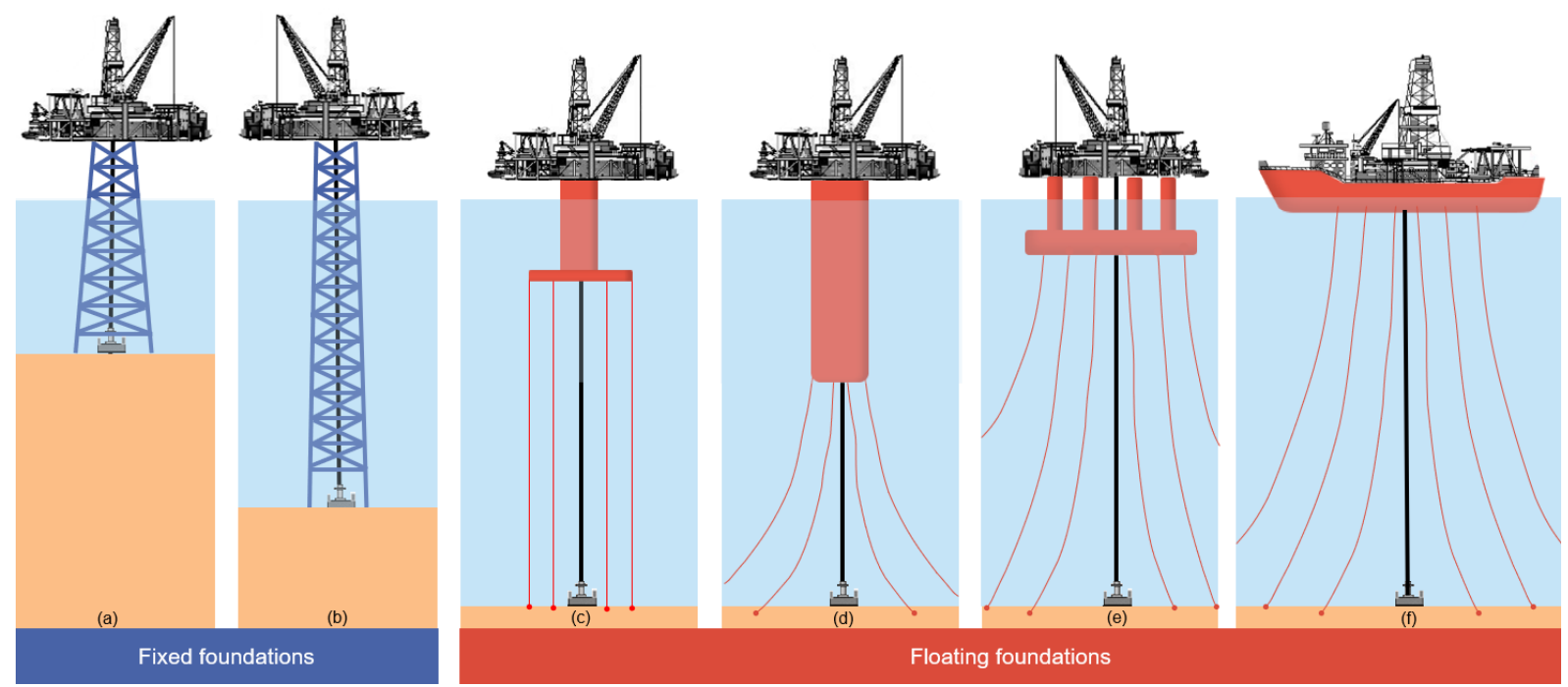

Source: The Author

The focus of the present master dissertation is to study the stiffness of the mooring systems of floating platforms. In this context, let us characterize and exemplify the main types of floating units, as follows:

- Spar Platform: consists of a large vertical buoy cylinder where the majority of the facility is installed above the sea water level, in order to increase the stability. Examples: Devil's Tower, operated by Eni (Dhanak and Xiros, 2016) (O\&G) and Hywind Norway, operated by Equinor (FOWT).

- Tension-leg platform: consists of a vertically moored floating structure. Examples: Magnolia, operated by Conoco-Phillips (Dhanak and Xiros, 2016) (O\&G) and GiconSOF, not operating yet (GINCON-SOF, 2018) (FOWT).

- Semi-submersible platform: consists of a floating structure with a large deck. The stiffness associated with angular motions on vertical plane arises from the columns water plane area, located bellow the sea water level (SWL) and also from the mooring system. Examples: NaKiKa, operated by Shell (Dhanak and Xiros, 2016) (O\&G) and OC4-DeepCwind, not operating yet (Robertson et al., 2014a) (FOWT). 
Figure 6 - Offshore Wind Turbines Platforms: (a) Gravity based, (b) Monopile, (c) Tripile, (d) Tripod, (e) Jacket, (f) Spar, (g) Tension-leg and (h) Semi-submersible.

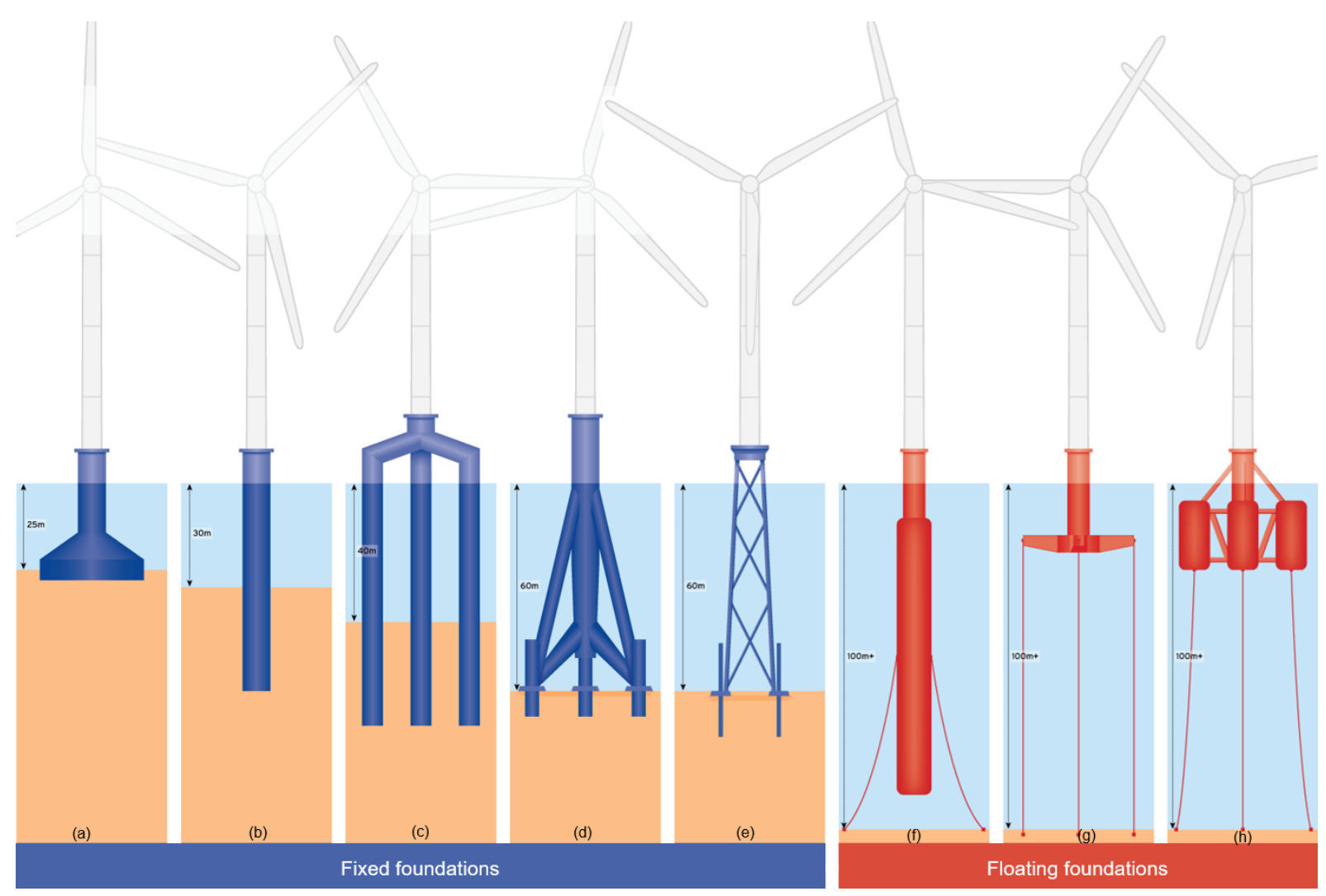

Source: Adapted from Wind Power Offshore (2013)

- Floating Production, Storage and Offloading unit (FPSO): consists of a floating vessel designed to product and process hydrocarbons and to stock the processed oil until offloading onto a tanker or a pipeline. Examples: Cidade de Anchieta and Cidade de Angra dos Reis MV22, both operated by Petrobras (O\&G).

\subsection{Motivation and Contributions}

As presented in the previous section, the design of mooring systems involves numerous variables. In this context, during the early stages of an offshore energy project, the designer has to choose among different mooring concepts in order to decide which to use. At this moment, highly nonlinear high-hierarchical simulations and model tests might not be the easiest way for iterative improvements on the design due to their high computational and development costs. Thus, analytics and expedite tools can drive this initial choice of parameters design.

This master dissertation brings a formulation that can help both the design and the analysis of mooring systems. This methodology is based on the formulation of the 
generalized restoring forces associated with the mooring system and makes use of classic Analytical Mechanics techniques. Thus, an analytic and explicit formulation for the stiffness matrix of the mooring system considering the six DoFs that characterize a rigid-body motion is provided.

The closed formulation herein proposed can be practical and useful for the design and analysis of mooring systems. As it is deeply detailed in the next chapter, the stiffness matrix of the mooring system is important for analytical, experimental and even numerical studies, and there is a lack of expedite formulations for its evaluation.

\subsection{Text Organization}

The current text is structured as follows. Firstly, a literature review of mooring systems design and analysis is presented, helping the reader to understand the main scientific developments in the study of mooring systems. This literature reviews also identifies open aspects, shedding light to the contributions of the research herein described as state-of-art. Secondly, Chapter 3 brings the mathematical model for the mooring system forces and the proposed formulation. A formulation for evaluating the mooring tension for a multi-segment mooring line is also presented in this chapter. The focus of Chapter 4 is to present real applications of the proposed tool in the form of case studies. Chapter 5 summarizes this dissertation, presenting conclusions concerning the applicability of the presented methodology. Finally, some Appendixes with important aspects of the development of this text are included. In particular, Appendix B brings another contribution of this work: a closed formulation for the static configuration of multi-segment mooring lines. 


\section{Literature Review}

This chapter presents a review of the scientific literature considered important for the development of this master dissertation. It is divided into four different sections, one for each of the most relevant topics. Firstly, an overview on the design and analysis procedures is presented in Section 2.1. Secondly, Section 2.2 brings proposals of design tools to better choose the mooring system parameters. These tools are based on different approaches, from analytical formulations to nonlinear numerical solutions (including Finite Element, FE, analysis). Thirdly, Section 2.3 discusses mathematical models for calculating loads (forces and moments) associated with the mooring lines. Last, Section 2.4 presents some models available in the literature for dealing with the evaluation of the stiffness matrix resulting from the mooring system. This section not only shows to the reader the actual scenario of this topic, but also clarifies the need for an expedite closed-formulation, the main contribution of the master dissertation.

Prior to the start of the literature review, an important aspect should be pointed out. Some references herein presented contain important concepts used in more than just one section. For example, Bernitsas and Garza-Rios (1996) present an inventive tool for the mooring system design based on an analytical methodology. The authors also bring up important considerations on the mooring restoring forces. In this scenario, Section 2.3 discusses the mooring forces model employed by the authors, while Section 2.2 presents the general idea behind the design tool.

\subsection{Mooring System Design and Analysis}

The design of a mooring system is an iterative process and it is intrinsically related to the analysis. Thus, systematic approaches and regulation codes should be used during a mooring system project. This section aims at presenting an overview on this iterative process, highlighting the main steps involved and the regulations, standards and rules related to the design and analysis. Ma et al. (2019) bring an example of mooring design and analysis workflow, reproduced in Figure 7.

Different international standards are available for design and analysis of mooring systems. These codes indicate criteria and recommendations for good and safety design, such as strength and fatigue design criteria. The most common design codes are:

- American Petroleum Institute (API, 2005) Recommended Practice 2SK "Recommended Practice for Design and Analysis of Stationkeeping Systems for Floating Structures"; 
Figure 7 - Typical mooring design and analysis workflow.

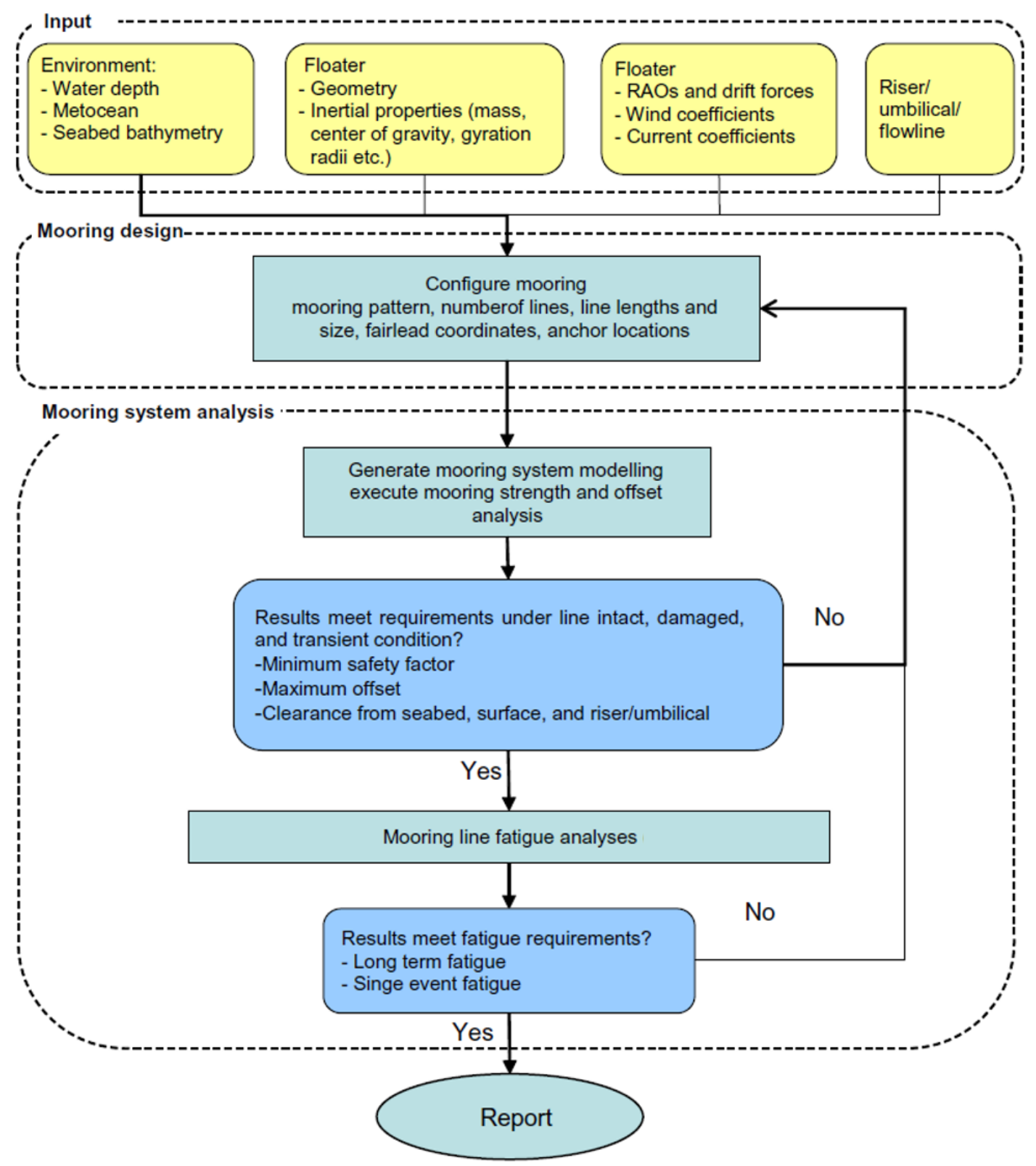

Source: Adapted from Ma et al. (2019)

- International Organization for Standardization (ISO, 2013) 19901-7 "Petroleum and Natural Gas Industries - Specific Requirements for Offshore Structures, Part 7: Stationkeeping Systems for Floating Offshore Structures and Mobile Offshore Units";

- Der Norske Veritas (DNV, 2010) DNV-OS-E301 "Position Mooring";

- American Bureau of Shipping (ABS, 2014) "Rules for Building and Classing Floating Production Installations";

- Bureau Veritas (BV, 2015) NR 493 DT R03 E "Classification of Mooring Systems 
for Permanent and Mobile Offshore Units".

The main information needed for the design of mooring systems are called design basis, normally given by the mooring system contractor. It outlines the most important factors to be considered for the mooring engineers during the project: environment, floater characteristics and other issues. It also gives the mooring system design main objectives such as: (i) the vessel offset limits and (ii) strength and fatigue life, under both normal and extreme operation conditions.

The mooring design starts with the definition of a preliminary mooring system configuration. In this context, it is common to refer to existing facilities. The first important variable that guides the project is water depth. Not only the choice of the mooring line profile and composition (see Table 1), but also the anchor radius is a function of it. Figure 8 shows the relation between anchor radius and water depth, based on the number of existing facilities. Obviously, other variables affect the selection of the anchor location, such as the seabed profile, location of subsea facilities, among others.

Figure 8 - Relation between anchor radius and water depth, based on the number of existing facilities.

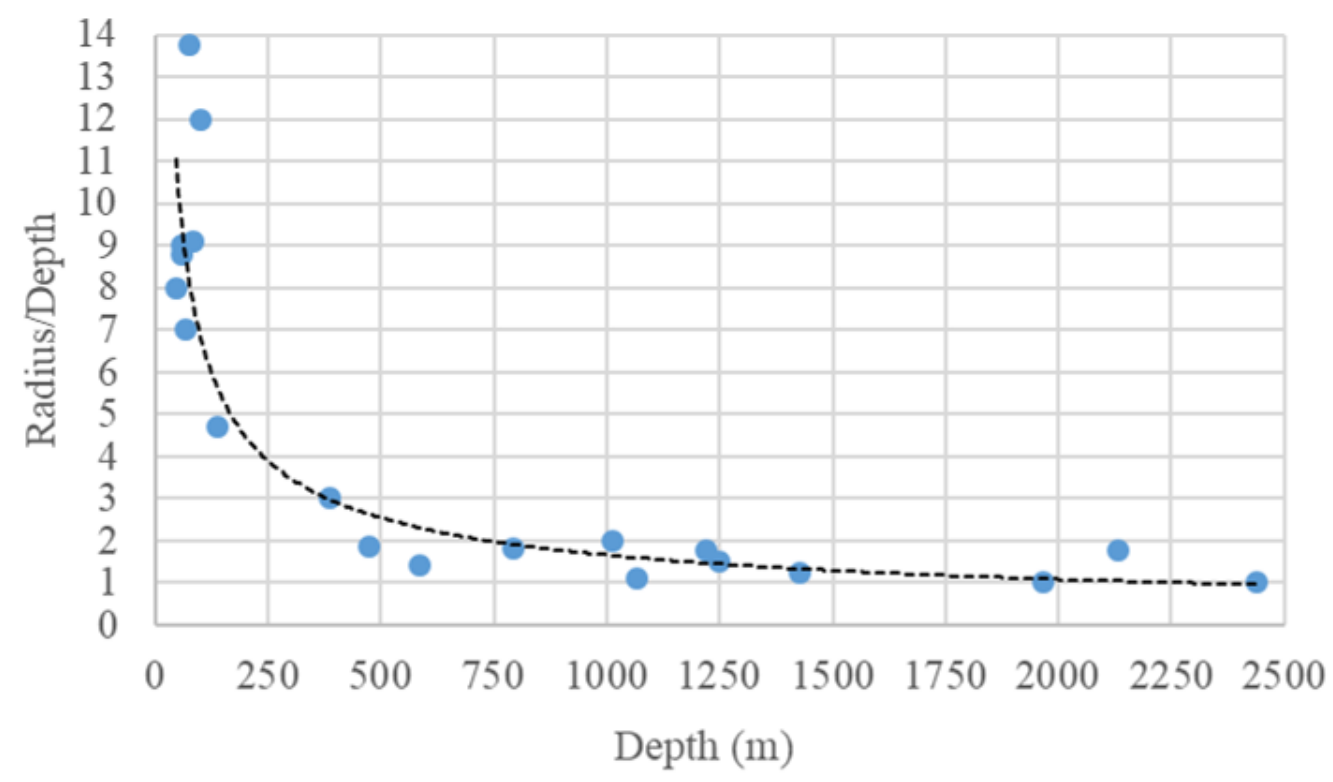

Source: Ma et al. (2019)

After choosing the proper mooring line profile and anchor radius for a given water depth, it is time to define the number and size of lines for the system. Some existing projects are normally used as reference for a initial configuration. Ma et al. (2019) emphasize that the number of lines should be as low as possible, provided the cost associated with this number. However, it is possible to increase the number of lines as the required line size 
becomes larger than the limit commercially available. The line pre-tensioning should also be taken into account while determining the number of lines. The pre-tensioning should be between $10 \%$ and $20 \%$ of minimum breaking load (MBL) and experimented mooring engineers normally keep pre-tensioning as low as possible (of course meeting the vessel offset requirement).

For SMS, other important variables to be determined include the grouping and the spread angles. The choice is function of the mooring pattern constraints, such as risers arrangement or the proximity of other installations. Grouped systems are preferred whith high number of lines, since they allow more open space for risers and better load distribution.

Ma et al. (2019) stress that at least four variables need to be tuned during the design process: vessel offset, line tension, fatigue damage and clash avoidance. These variables are evaluated during the mooring analysis stage. The authors propose some options for a proper choice of the mooring systems. The variables and the respective alternatives are presented below.

The main objective of the mooring system is the vessel stationkeeping. Risers and umbilicals are structures very sensitive to offsets. Generally, taut-leg lines and choosing line material of high tensile stiffness can help reducing the vessel offset. Some other actions can be taken, such as: increase line pretension, increase the number of lines, use lightweight materials to minimize the catenary effect, use clump weight or heavy chain at the touch down zone for hybrid mooring lines, arrange line spread in the direction of extreme environment.

A good mooring design should not result in high line tension. The line tension can cause different kinds of integrity issues. Large line diameter and a proper mooring line static configuration can help to make tension safety factor meet industry and classes standards, i.e. the mooring line tension is kept within the design limit. Some alternatives for minimizing the tension in the line include the choice of a proper mooring profile for the water depth, the increase in the number mooring lines, consider the direction of extreme environment for optimal line spread choice, and the choice of lighter and less-stiff line material such as, for example, polyester.

Another important design parameter is the fatigue life. It should exceed the field life, considering a proper fatigue safety factor since this has been one of the most important mooring line failure modes. Among the most employed materials (as aforementioned, chain, wire and rope), chain is the most susceptible to fatigue failures. In order to improve the fatigue life, the designer can (Ma et al., 2019): reduce dynamic tension (by improving the hull design), increase the line size, properly design the fairleads or choose other materials than chain. 
Finally, the design should predict the avoidance of clash. The mooring system has to accommodate different subsea infrastructures, without any interference. The line can not collide with the hull either. Some actions to avoid clash include: change spread angles, reduce anchor radius, change mooring line profile or include buoys.

As abovementioned, the design is intrinsically related to the analysis of a mooring system. Indeed, as the design is an iterative process, the proposed mooring system should always be verified during the analysis phase. In this scenario, Ma et al. (2019) list different possibilities that can be used for a proper mooring analysis: quasi-static or dynamic analysis, frequency domain or time domain analysis, uncoupled or coupled analysis among others. These possibilities and the differences between then are presented in the following paragraphs.

The authors indicate that the main difference between the quasi-static and the dynamic analysis is the treatment of the wave frequency responses of the floater. Kwan and Bruen (1991) state that the dynamic analysis takes into account the effects from the line added mass and hydrodynamic damping. Furthermore, the quasi-static analysis ignores these effects and the tension distribution is function of the fairlead position only. Ma et al. (2019) say that, despite being less precise in terms of the hydrodynamic effects, the quasi-static analysis is much simpler than the dynamic analysis. The authors affirm that the latter is appropriate for computing mooring line responses due to both the mean offset and the low frequency motions, while the former is needed for vessel responses to wave frequencies. Figure 9 illustrates the difference between the quasi-static and the dynamic analyses.

On the one hand, the frequency domain analysis is commonly employed due to its efficiency (in the computational point-of-view) when compared to time domais analysis. It assumes that the response can be written as static and frequency dependent, and that superposition approach is valid. Thus, the vessel motions are divided into four different components: mean, wave frequency, low frequency and high frequency, the latter is normally neglected. Ma et al. (2019) point that the limitations of the frequency domain analysis are: (i) the equations of motion are linearized, (ii) the extreme value distribution is not directly achieved from the analysis and (iii) the combination of the wave and low frequency responses is empirical. On the other hand, the time domain analysis is more time-consuming, as it solves the floater and mooring system responses through numerical integration. According to the same authors, this analysis is able to account different nonlinearities, such as: (i) geometric nonlinearity, associated with a instantaneous deformed configuration significantly distinct from the reference, (ii) contact between line and seabed and (iii) fluid-structure interaction.

The mooring analysis can also be uncoupled or coupled. The latter is traditionally the most common provided the former is more time-consuming. An uncoupled analysis 
Figure 9 - Quasi-static and dynamic mooring analyses.

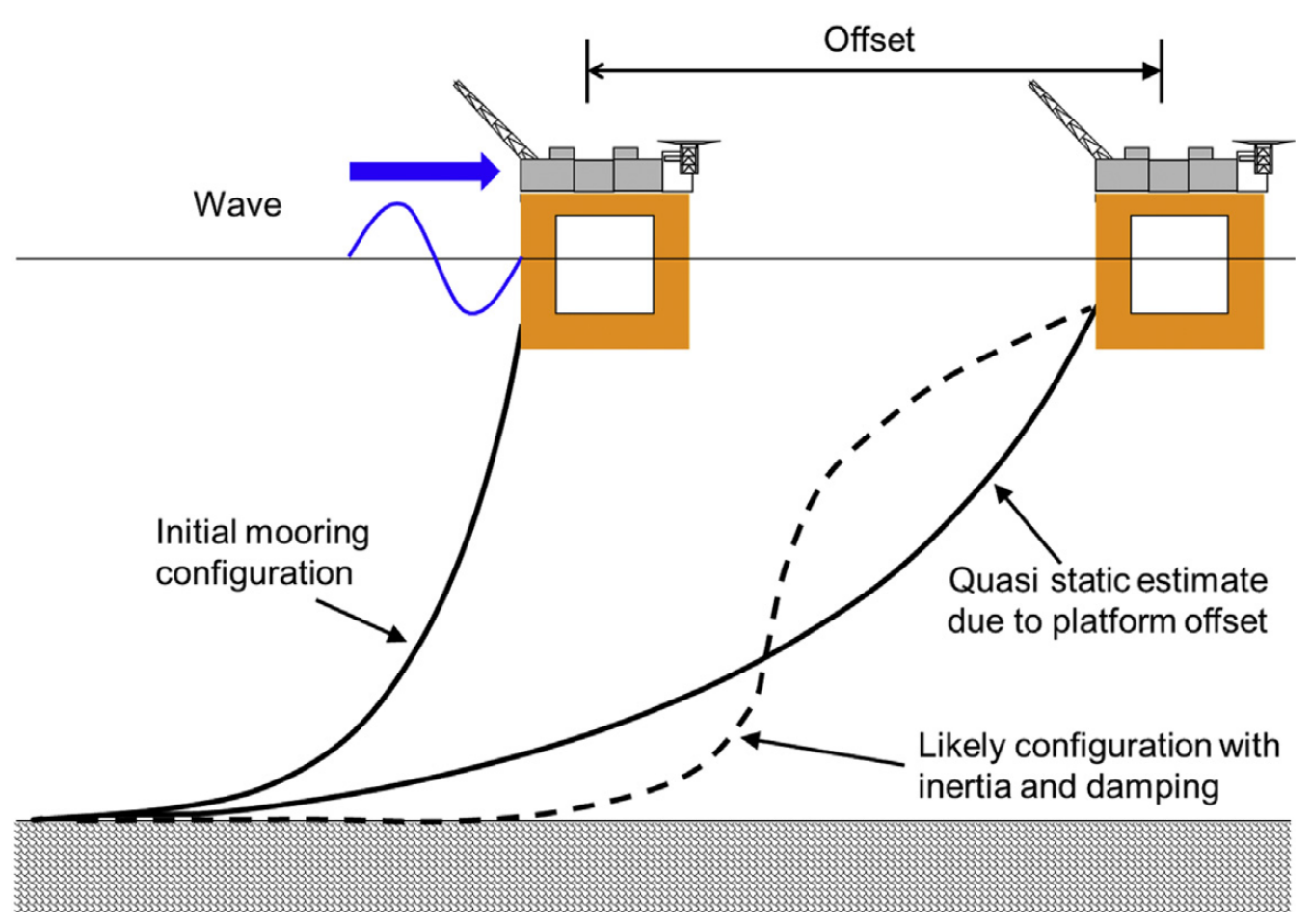

Source: Ma et al. (2019)

computes the floater motion analysis and the mooring analysis separately. The motion analysis considers simpler mooring line models - through its stiffness matrix, for example and the output is used as input for the mooring analysis. Webster (1995) says that the mooring system dissipating forces can be linearized and translated as an induced damping, also called mooring damping. Ma et al. (2019) claim that the uncoupled analysis yields three important simplifications: (i) the mooring system dynamics does not affect the vessel motion, (ii) the dynamic dissipating effects are linearized as a mooring-induced damping and (iii) sea current forces on the mooring lines are neglected. For a coupled analysis, both the vessel and mooring responses are simultaneously solved. Thus, all the dynamic effects can be calculated. This approach is more necessary in deep and ultradeep waters, as the coupling between vessel motions and mooring responses is more important. In the industry, the uncoupled analysis is largely used and it is a valuable tool when well validated, mainly for early stages of the design process. In turn, the coupled approach is more accurate and, from the theoretical point-of-view, correct. Hence, it is normally used as design verification for governing load cases.

Notice that many are the factors to be taken into account during a mooring system design and analysis. Thus, the scientific community has been thoroughly engaged on the study of this area. The next section brings some of the most recent advances. 


\subsection{Mooring System Design Tools}

Even though widely studied, some effects of the mooring system on the response of the floating body are still under study. Additionally, due to the numerous variables involving the project, analytic methods are of great value for understanding the relation between the design parameters and the static and dynamic behavior of a moored system. In this context, researchers have been proposing classic and innovative nonlinear dynamic methods to investigate this interaction. The current section aims to present some of these recent research efforts.

Triantafyllou (1982) brought an analytical inventive tool for preliminary design of mooring systems based on an iterative procedure. The author proposed a flow-chart that can help find the optimal design solution. The simple process starts with an assumption of the cable properties. Under static forces such as sea current, the static solution is calculated. The dynamic solution is then investigated, considering low-frequency oscillations caused by the second-order wave forces. This whole process is repeated until the optimal solution is found. Figure 10 brings this flow-chart.

Figure 10 - Overall design procedure.

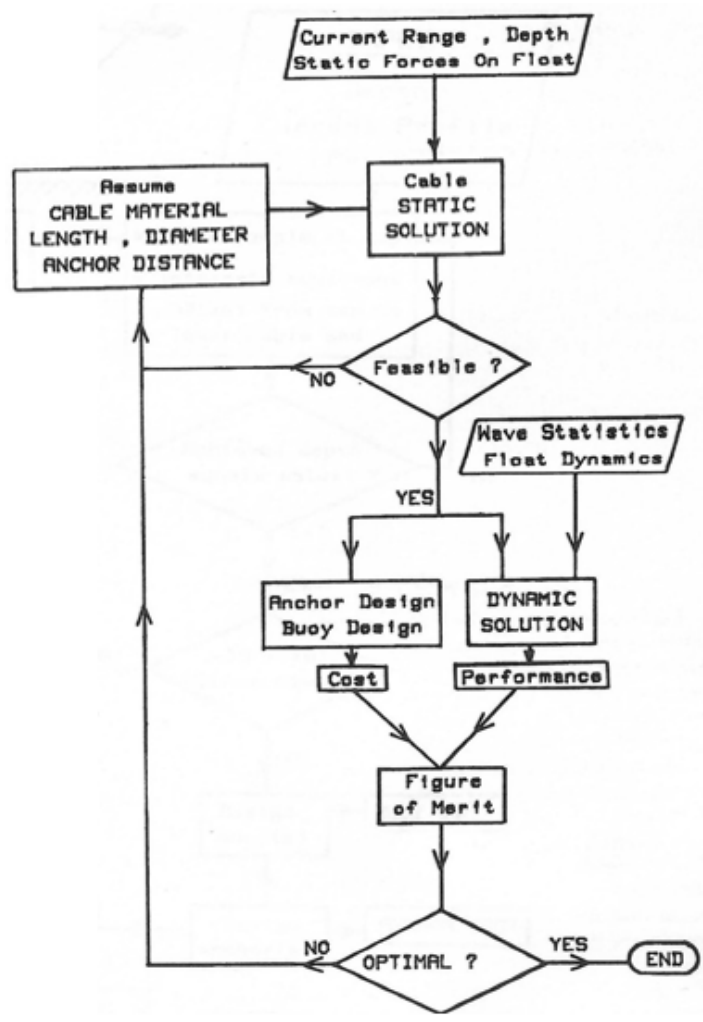

Source: Triantafyllou (1982)

Pesce (1986) also proposed a preliminary analytical design tool. The author developed a linear analysis of the moored vessel around an static equilibrium position. In fact, 
the author proposed a stiffness matrix for the vessel planar degrees-of-freedom (DoF) in order to calculate the response of the system to mean drift excitations.

Bernitsas and Garza-Rios (1996), Bernitsas and Kim (1998), Garza-Rios et al. (2000) and Garza-Rios and Bernitsas (2001) proposed investigating the mooring design parameters effects on the dynamic response of the moored system using a catastrophe set methodology. This innovative powerful tool is of great value for a preliminary design, once it helps the basic understanding of the relation between design parameters and the stability of the system.

The catastrophe set methodology is based on the stability analysis of the equilibrium points. The dynamics on the horizontal plane problem is initially a three-dimensional configuration space, with the generalized coordinates being the two horizontal translation motions and the rotation around the vertical axis. Then, the state space representation is a six-dimension space for the autonomous problem or seven-dimension space for the nonautonomous one. The stability of the equilibrium position is investigated when the system is autonomous (i.e. not time dependent) by means of the Lyapunov's indirect method. For the nonautonomous systems (such as when the external forces are time dependent), the stability is qualitatively investigated via numerical integration of the nonlinear equations of motion. The systematic variation of some mooring system parameters allows investigating the stability of the equilibrium points and bifurcations. The results of this investigation are plotted in the form of catastrophe sets.

Bernitsas and Garza-Rios (1996) developed a parametric study to investigate the influence of the SMS line position on the response of the moored vessel. The effects of the pre-tensioning, the fairlead position with respect to the symmetry axis and angle of each mooring line were investigated, as well as its immersed weight per unit length. It was demonstrated that, depending on the configuration of the mooring lines, different responses appear, namely, stable and unstable equilibrium positions, limit cycles and even chaotic dynamics.

Bernitsas and Kim (1998) investigated the effect of the mooring system configuration on the response of the moored vessel under slow-drift excitation forces. Again, the catastrophe set methodology was used to guide the designer to chose the geometric parameters of the mooring system.

Garza-Rios et al. (2000) presented the effects of hybrid mooring lines. The authors discussed that for deep water, the weight of chain mooring lines induces a vertical force on the moored body. The proposed alternative is to consider hybrid synthetic rope-chain line. Each mooring line is composed of a long synthetic rope connected to chain segments on both extremities. The authors formulated the horizontal and vertical fairlead-anchor distance as functions of the horizontal component of the tension at the fairlead and the distance between the end of the bottom chain to the touchdown point (TDP). In order 
to illustrate the effect of the elastic-stiffness of the rope on the response, the parametric study developed considers also three different synthetic ropes. The authors compared different materials, namely nylon, polypropylene and polyester. Three mooring system arrangements were under investigation: a turret single point mooring with four lines, a four-line DICAS and a ten-line DICAS. The water depth was also parametrized in order to evaluate its effect on the stability of the equilibrium of those systems. The catastrophe sets were evaluated for different values of the rope angle and the pre-tensioning of the line. The charts show that variations in mooring line angles and pre-tensioning could yield loss of static stability for DICAS and dynamic loss of stability for turret single-point mooring systems. In other words, the vessel's horizontal plane dynamic response can be improved by increasing the pre-tensioning and reducing the line inclination. However, the authors remarked that an increasing of mooring tension can also affect the vertical motions. A good mooring system project also has to consider the horizontal dynamics with the vertical motions. In addition, the water depth seemed to have important effects on the dynamic stability of single point mooring systems and static stability of DICAS.

It is important to remark that, considering synthetic rope moorings, Flory, Banfield and Petruska (2004) explained that some important properties of a mooring line may change with the elongation caused by the vessel motion. In this context, they proposed a design analysis considering the maximum dynamic offset during storm conditions.

Garza-Rios and Bernitsas (2001) gave continuity to the previous works with catastrophe sets, but investigating the effect of size and position of intermediate buoys on the mooring system. Again a parametric study was developed, varying both the buoy parameters and the water depth. A 4-SMS-2 was taken as a case study. The equations of motion included each buoy motions, increasing the dimension of the state-space. The authors demonstrated that variations in the buoy design parameter can also yield loss of static and dynamic stability (and even lead to chaotic responses).

In order to exemplify the catastrophe set method, some results of Bernitsas and Garza-Rios (1996) are presented. The charts are plotted for dimensionless position of the fairleads with respect to the vessel length and full line length. Figure 11 presents the nonlinear time domain response of a three-line SMS tanker without propeller whereas Figure 12 brings the catastrophe set for this problem. The only external force is the autonomous sea current force, acting on the direction of the length of the vessel. Figures 12(a) and 12(b) refer to points G6 and G7, respectively. The catastrophe set indicates how the mooring parameters influence the response of the system. Between G6 and G7, the only difference is the increase on the total length of the mooring lines, which decreases the pre-tensioning of the lines. It is notable that the stable focus in Figure 11(a) is no longer stable in Figure 11(b). This result is well recovered through the catastrophe set from Figure 12. From the catastrophe sets, the authors concluded that the dynamic behavior of 
the moored system depends strongly on the design parameters, specially the mooring line pre-tensioning.

Figure 11 - Nonlinear time domain response.

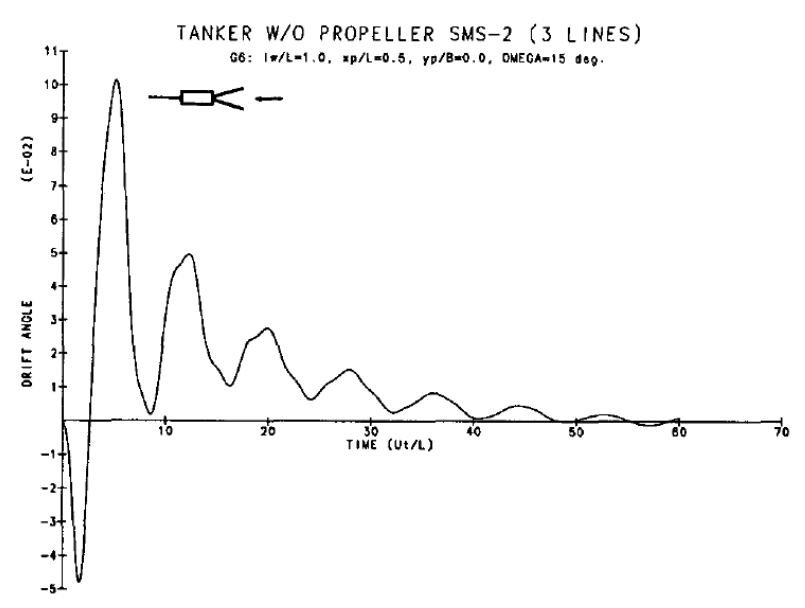

(a) Point G6.

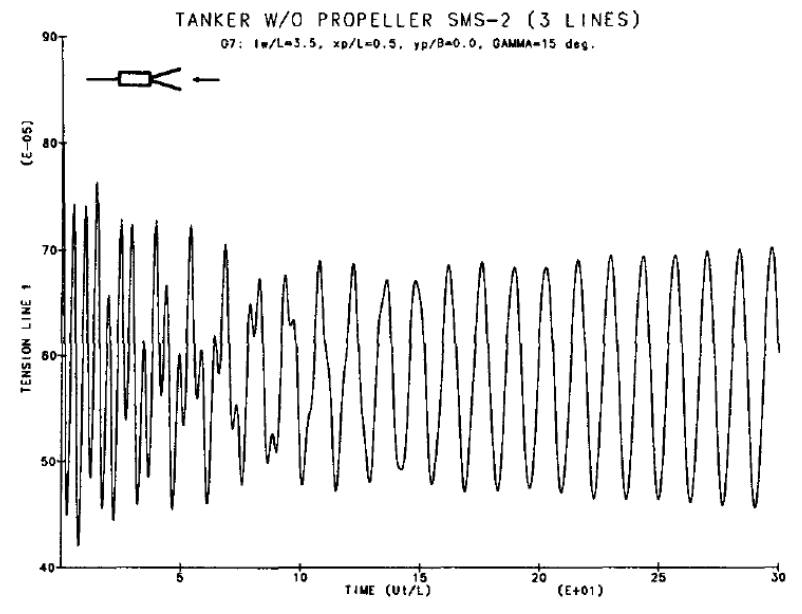

(b) Point G7.

Source: Bernitsas and Garza-Rios (1996)

Figure 12 - Catastrophe set.

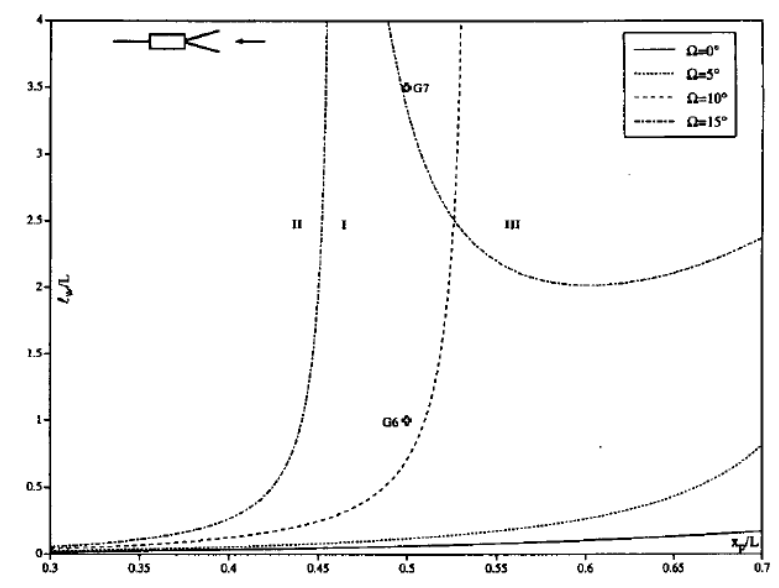

Source: Bernitsas and Garza-Rios (1996)

Brommundt et al. (2012) proposed a tool for optimized mooring system design for FOWTs based on a frequency domain analysis. This numerical analysis was applied to determine the linear response of the six degrees-of-freedom motions. In this scenario, the static equilibrium position of the platform was found considering the mean forces due to wind and sea current and the linearized stiffness matrix around the trivial equilibrium position. Then, the authors calculated the responses for dynamic loads from wind sea, ocean swell and turbulent wind. The optimization tool takes advantage of this initial 
linearized results to indicate a good preliminary mooring system design. The tool is based on the Nelder-Mead simplex algorithm to minimize the cost (related to the cables length) with the following design constraints:

- The mooring system has to support the ultimate load conditions;

- The load at the anchors should be strictly horizontal, i.e. a portion of the mooring lines have always to lay on the seabed;

- The motions of the platform have to stay within specified limits.

The process follows the same initial steps, considering primary design line parameters such as radius to anchors, length and angle of mooring lines. These parameters are determined by the optimization procedure. The direction of each environmental load is taken into account. The final design might lead to an asymmetric mooring system. Then, the proper definition of load cases and direction is crucial for the correct final design.

Montasir, Yenduri and Kurian (2015) investigated the effect of the mooring system configurations on the dynamic responses of truss spar in surge, heave and pitch. Two line arrangements were compared, considering the number of mooring lines groups and the number of lines in each group: three groups with three mooring lines and four groups with four lines. Different line angle scenarios were also considered. The responses were evaluated through numerical time domain integration. The mooring lines were modeled on a quasi-static scheme and the horizontal and vertical fairlead-anchor distances were defined as functions of the horizontal tension component and the line angle related to horizontal at the fairlead. The responses were evaluated in the form of Response Amplitude Operators (RAO). After numerical studies, the authors drew conclusion on the effect of the number and the symmetry-asymmetry of the mooring system on its static restoring capability and the dynamic response of the platform.

Campanile, Piscopo and Scamardella (2018) proposed a tool for the mooring design and selection for FOWTs in intermediate and deep waters. The process is based on ultimate (ULS), accidental (ALS) and fatigue (FLS) limit state conditions. The six-DoF static and dynamic offsets were determined in the frequency domain, considering first and second order motions. It was considered that the maximum displacement had to be within an admissible limit. The maximum displacement was the sum of the mean static and the dynamic offset. The later was evaluated as a combination of significant and maximum offsets caused by wave-frequency or low-frequency oscillations or both ${ }^{1}$. The significant responses were calculated using classic frequency domain equations. Figure 13 presents the flow-chart for the mooring system design. Finally, the authors proposed also a preliminary

1 These oscillations are caused by first and second order wave forces. For further details, see Journée and Massie (2001) 
cost analysis considering both offshore and onshore maintenance inspections. Couple recommendations are then made: redundant mooring systems (i.e. systems with more than just one line in each direction) should be considered regarding potential failures; the platform admissible offset has to be tuned to minimise mooring costs. The authors also made comments on FOWT farms.

Figure 13 - Flow-chart of mooring system design.

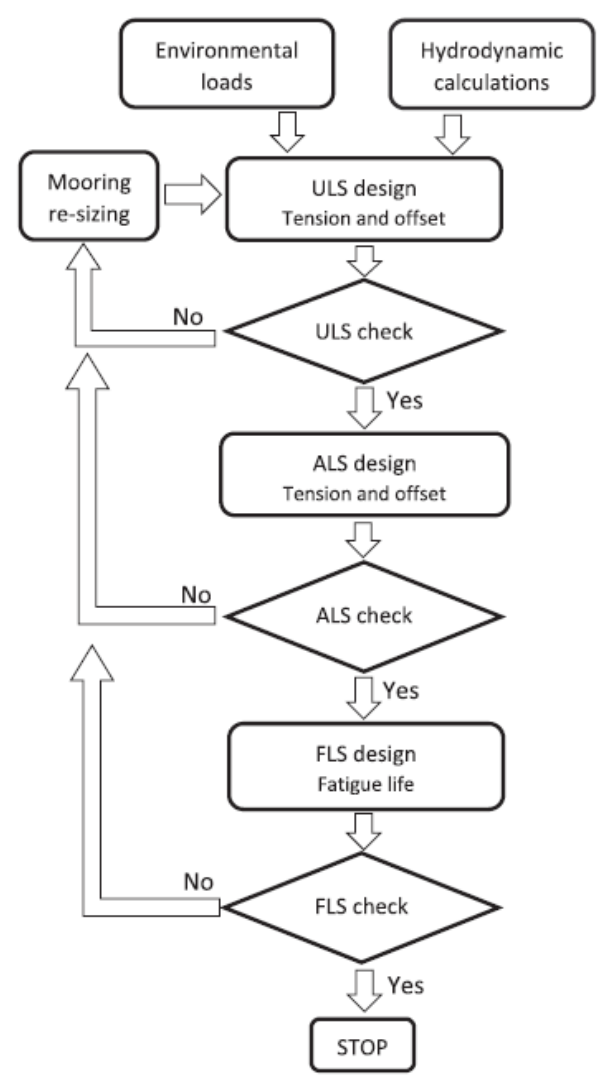

Source: Campanile, Piscopo and Scamardella (2018)

\subsection{Mooring Restoring Forces}

The investigation of the mooring restoring forces is a classical and important research topic. In fact, even though the classic inextensible catenary line solution is well known, researchers have been proposing improvements in order to consider the line extensibility, the contact with the seabed and other mooring line configurations. Additionally, a number of contributions have proposed different (but not necessary incompatible) ways to compute the mooring loads. The goal of this section is to discuss some of the mooring line and system restoring forces available in scientific literature. This way, some of the formulations are herein presented. 
In the early work of Yamamoto, Yoshida and Ijima (1980), the authors studied the two-dimensional problem of a moored floating body. The mooring lines were considered as linear springs, with an arbitrary defined spring stiffness $K_{i}$. Therefore, mooring forces are just linear spring forces.

Oppenheim and Wilson (1982) presented a parametric study for the choice of polynomial approximation for the restoring mooring systems forces on the horizontal plane. First, the authors defined the relation of the horizontal component of the tension at the fairlead and the fairlead-anchor horizontal distance for a multi-segmented mooring line. Regarding force and displacement compatibility, they considered each segment as catenary laying lines. Then, the effect of each mooring line was considered in order to define generalized forces. The resultant restoring force was considered by projecting each horizontal tension at generalized directions. Finally, the authors approximated each of the restoring forces to third-order polynomials by using the least-square fit and the MonteCarlo method. The conclusion was that, in some cases, results with satisfactory accuracy were achieved. Hence, it seems that for early design stages those approximations could lead to more practical applications than using the full nonlinear mooring system forces.

Pesce (1986) proposed a formulation for generalized restoring forces for a symmetric moored vessel in an equilibrium configuration. This model is generic for any mooring line static configuration. The generalized forces are projections of the horizontal component of the mooring tension at the fairlead. It is important to point out that the formulation presented in Pesce (1986) deals with loads on the horizontal plane.

Bernitsas and Garza-Rios (1996) and Bernitsas and Kim (1998) considered in their studies different mooring line forces, from classical catenary formulations to taut-leg lines. The model was addressed for the horizontal-plane constrained problem. The authors consider semi-empirical equations for taut-lines forces based on line average breaking strength, rope length and working length. The investigated SMS is sketched in Figure 14. The restoring mooring system forces are, once again, geometrical projections of the forces on the fairlead.

In another work, Garza-Rios et al. (2000) presented a hybrid mooring line model for a SMS, again for a problem on the horizontal plane. The model had three segments: a bottom catenary laying chain, a synthetic rope taut line and a upper taut chain. Figure 15 brings a sketch of the mooring line configuration. In this context, the authors computed each mooring line force through the compatibility of each segment's forces and displacements. The mooring system horizontal generalized forces were computed similarly to the previous works of Bernitsas and Garza-Rios (1996) and Bernitsas and Kim (1998).

Garza-Rios and Bernitsas (2001) formulated the equations for a system with an intermediate buoy. The authors computed the compatibility of forces and displacements, considering both line segments as catenary laying lines. Figure 16 illustrates the geometry 
Figure 14 - Sketch of a four line SMS.

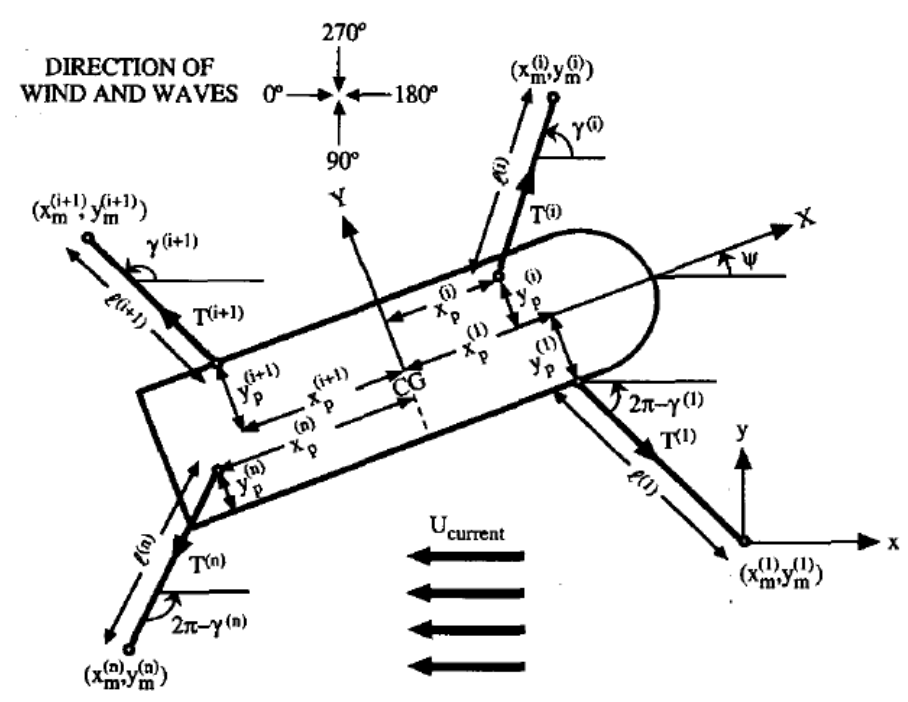

Source: Bernitsas and Garza-Rios (1996)

Figure 15 - Geometry of hybrid line.

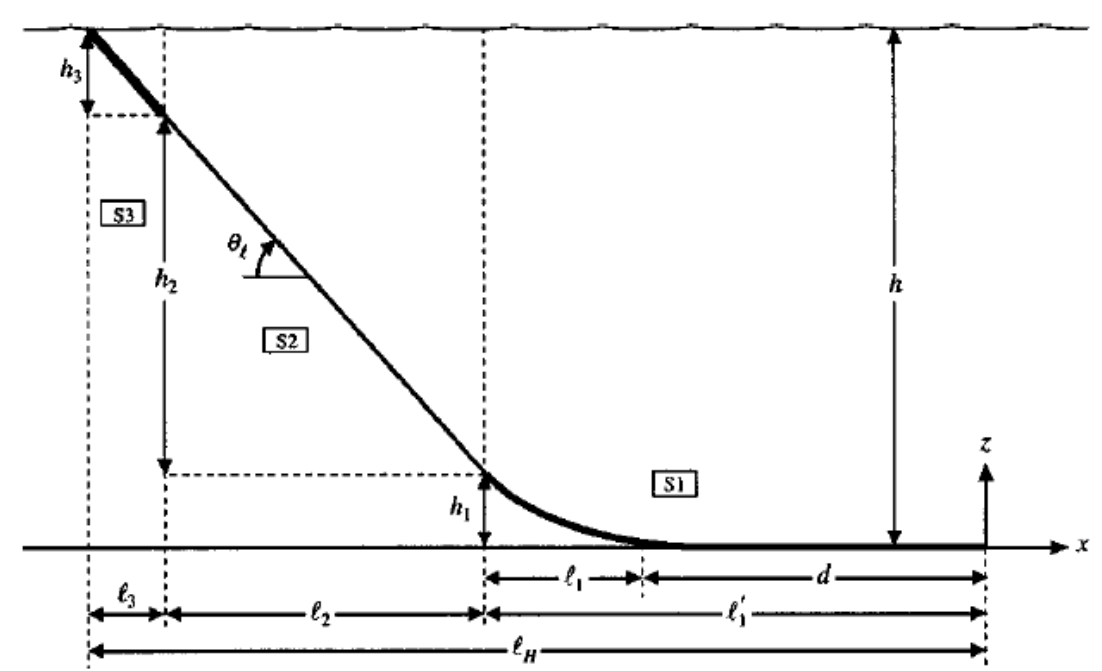

Source: Garza-Rios et al. (2000)

of the buoy-supported lines. The SMS generalized forces on the horizontal plane were calculated. It is important to remark that, in any of the aforementioned works, the effect of the catenary laying line extensibility was not considered.

Agarwal and Jain (2003) studied a three-segment mooring line with a clump weight, considering all possibilities for positioning the clump weight (i.e. completely laying on the seabed, partially laying on the seabed or completely suspended). The authors investigated the effects of the elasticity of the line to compute force-displacement relation. Figure 17 
Figure 16 - Geometry of buoy-supported line.

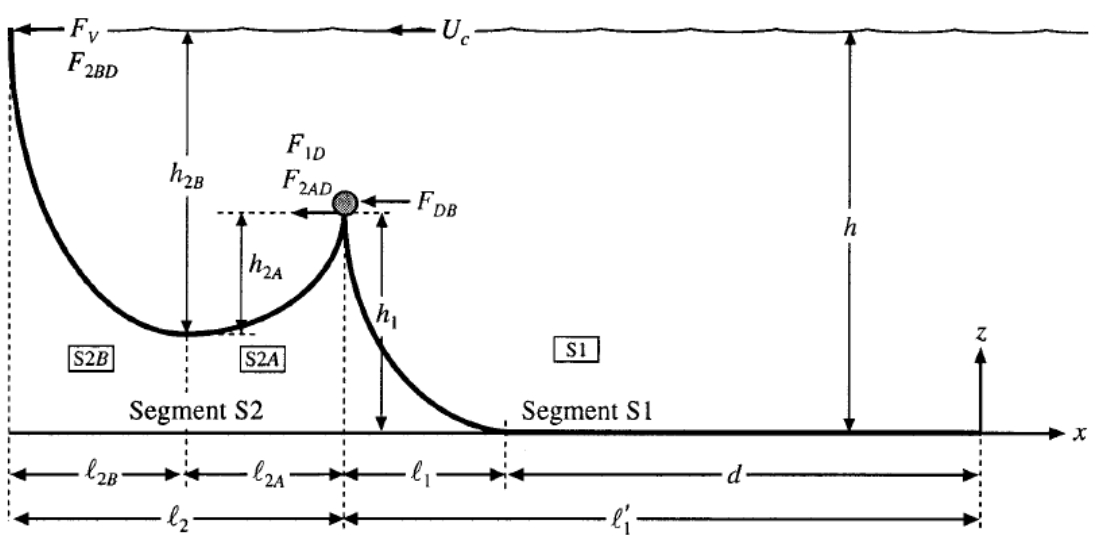

Source: Garza-Rios and Bernitsas (2001)

depicts the sketch of the line model. In order to consider the effects of the whole system on the restoring forces, the authors took advantage of the studied platform's symmetry to model the horizontal forces only once (i.e. generalized forces in surge and sway directions were modeled only once). Vertical force is presented, also. Controversially, the authors did not presented any formulation for generalized moments. The SMS generalized forces at the horizontal plane were calculated projecting the forces at generalized directions.

Figure 17 - Three segment mooring line with a clump weight.

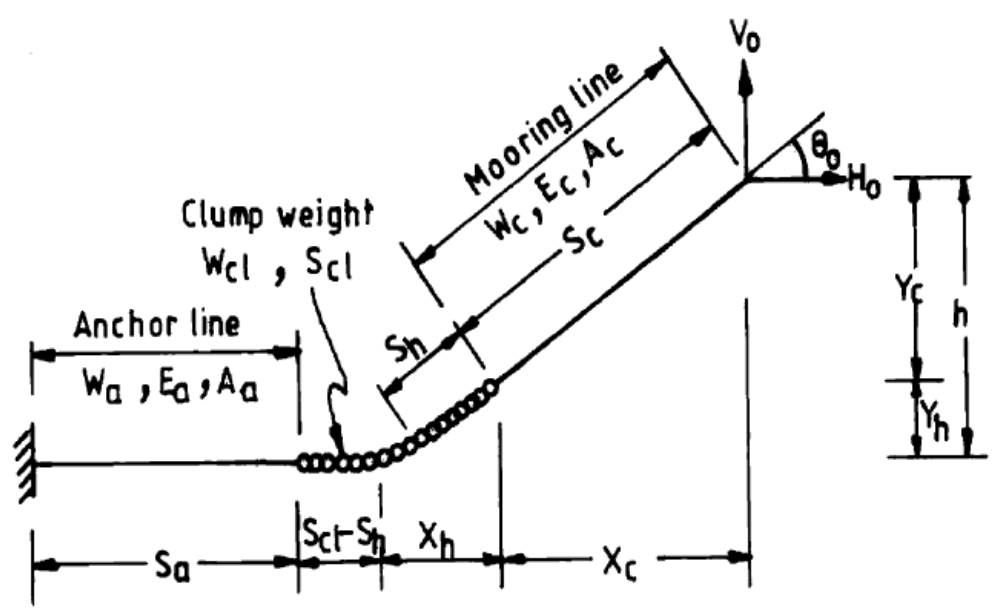

Source: Agarwal and Jain (2003)

Umar and Datta (2003) have proposed a polynomial approximation for generalized restoring forces. The line model was a classic inextensible catenary one. The authors studied an one-DoF oscillator, considering only the translational response of a moored buoy due to a external force in that direction, e.g. surge $(x)$. The restoring force $r(x)$ was 
calculated following the same proposal of Oppenheim and Wilson (1982), but it was a 5th order polynomial approximation.

Loukogeorgaki and Angelides (2005) considered the effects of mooring lines on a six-DoF system. Considering a classic catenary mooring line, the authors computed the generalized restoring forces by projecting the tension at the fairlead on each generalized coordinate direction. As shown in Chapter 3, the present master dissertation also brings a formulation for the mooring system stiffness matrix. However, the latter is derived for any generic position, using classical approaches from Analytical Mechanics.

Jonkman (2007) used an "extensible"-catenary ${ }^{2}$ mooring line. In this context, the author re-formulated the classic catenary equations in order to compute both the extensibility of the line and the friction between line and seabed. Restoring forces due to the complete mooring system was calculated by transforming the tension in each line from local mooring coordinate system to a global one and, then, computing all mooring lines' tension.

Masciola, Jonkman and Robertson (2013) presented a multi-segment, quasi-static cable model. This model takes into account a line that not necessarily lays on a vertical plane. Indeed, the authors have considered lines with more than just one anchor. Figure 18 illustrates a generic geometry mooring line. This model is based on the extensible catenary equations and allows to calculate force-displacement relations.

Al-Solihat and Nahon (2015) discussed three different formulations for mooring lines: a suspended catenary line (without any portion in contact with the seabed), a catenary line with a segment resting on the seabed and a taut line. For the three cases, the six-DoF restoring forces were computed considering the geometric relations between the mooring lines and the vessel elementary displacements.

Finally, Pesce, Amaral and Franzini (2018) proposed the evaluation of generalized restoring forces of the mooring system for the horizontal-plane problem following classical methods of Analytical Mechanics. The formulation took into account the horizontal force-distance relation for each mooring line and local generalized directions.

In closing it is possible to make many different models of the mooring line horizontal force-displacement relation, depending mainly on the line configuration. In general, mooring line forces are modeled as functions of both horizontal and vertical anchor-fairlead distances. Additionally, on the reviewed literature, generalized mooring system restoring forces are computed by simply projecting the tension at the fairlead, in a geometric relation. In Chapter 3 it is brought an expansion of Pesce, Amaral and Franzini (2018) methodology for the six-DoF problem . The formulation allows to easily linearize the generalized forces around any generic position, resulting in the stiffness matrix presented also in Chapter

$\overline{2}$ The term "catenary" is commonly used for inextensible cables, i.e. the cable extensibility is neglected. 
Figure 18 - Generic geometry of a multi-segment mooring line.

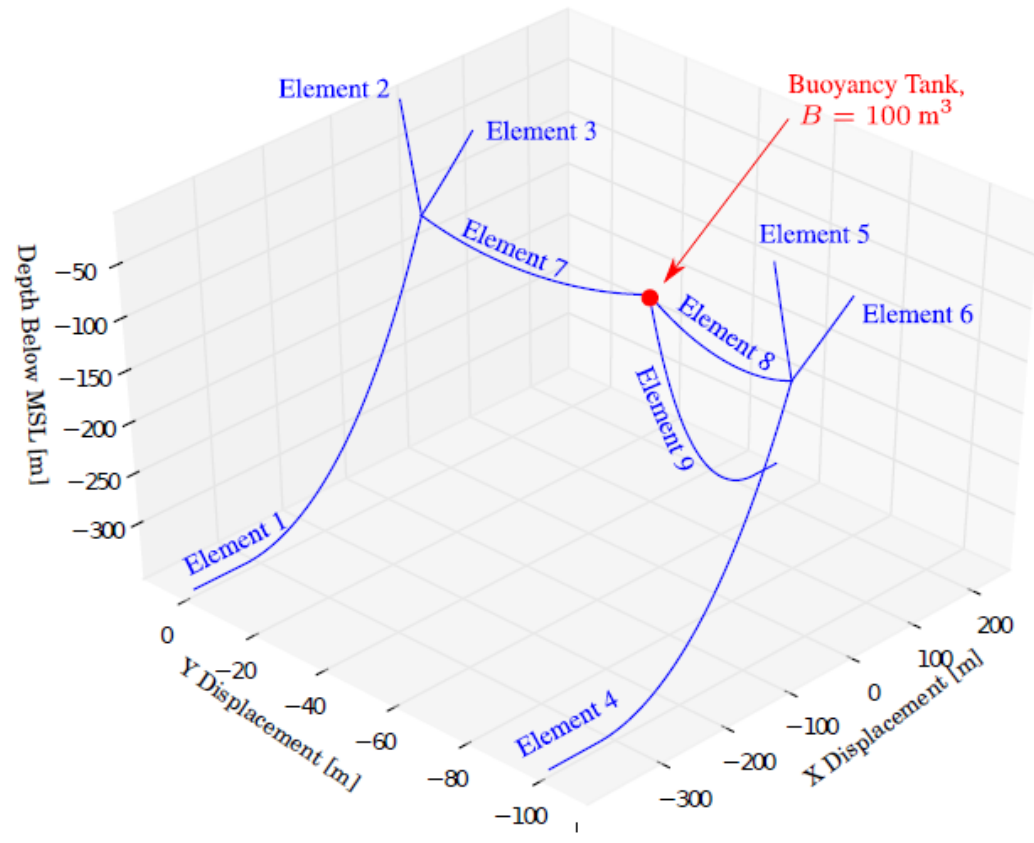

Source: Masciola, Jonkman and Robertson (2013)

3, the main contribution of this work. Higher-order polynomial expansions can also be developed. Although interesting, these nonlinear analysis is left for further work.

\subsection{Mooring System Stiffness Matrix}

The stiffness matrix due to the mooring system is a relevant information for any successful moored floating unit design. Indeed, the stiffness coefficients are of great value not only at initial stages but also for some case tests during the verification phase of the design. For instance, knowing the mooring stiffness matrix is mandatory whenever slow drift motions are concerned, either being the classical, on the horizontal plane (surge, sway, yaw), or those that might occur in heaving, pitching and rolling motions. As it is well known, slow drift motions are resonant responses of the system, at their low natural frequencies, to the action of second-order wave forces in low frequencies, the action of wind forces or even current induced forces, for which the mooring stiffness plays an important role. The mooring system stiffness matrix may also influence calculating the motion frequency response of the vessel to first-order wave forces, commonly named as RAOs. In this context, the scientific community has lately been working on the evaluation of the mooring system stiffness matrix. The present section brings a few relevant works on this topic.

First of all, it seems interesting to refer to an international standard. BV (2015) 
brings the requirements for the classification of mooring systems of floating units among others. According to the standard, the $6 \times 6$ stiffness matrix associates elementary displacements applied to the floating unit with the elementary reaction loads resulted from them, around a given position. It also stresses that the stiffness matrix can be obtained by a regression of the mooring forces time series versus the unit motions time series at low-frequency and wave frequency motions. Considering this, many authors have proposed different ways to evaluate the mooring system stiffness matrix.

From the reviewed literature, it is remarkable that authors have been proposing different partitions of the complete $6 \times 6$ stiffness matrix. Indeed, depending on their needs, some authors prefer to study a two-dimensional problem either on horizontal or vertical plane. Some others prefer to study only the stiffness matrix related to translational displacements. These approaches are justifiable by the particular application of each study. It is common to consider horizontal plane motions when the focus of the study is effects of waves second-order forces on the vessel motion. In turn, couplings surge-pitch (or even sway-roll) are only accessed via a two-dimensional vertical plane modeling. The use of the translational partition can be justified for a iterative process in order to estimate mooring system forces.

Yamamoto, Yoshida and Ijima (1980) have proposed a two-dimensional vertical plane stiffness matrix. The mooring lines were considered as linear springs with arbitrary spring constant and initial length, tension and angle with respect to the horizontal. The generalized coordinates were the horizontal in-plane displacement, the vertical displacement and the angular displacement with respect to the horizontal. Stiffness coefficients were obtained for a given hydrostatic equilibrium, where the coordinates of the center of gravity were known a priori. Then, for each mooring line, the stiffness coefficients were obtained by elementary static procedures for the vessel's motions. Figure 19 presents the sketch of the problem investigated by Yamamoto, Yoshida and Ijima (1980).

Based on the author's generalized restoring forces formulation, Pesce (1986) proposed a mooring system stiffness matrix for the planar problem. In this context, the author differentiated the forces with respect to the generalized coordinates, i.e. the horizontal plane translations and rotation with respect to a vertical axis, with considerations on the small displacements and rotations. Figure 20 brings up small displacement and rotations sketchs. The author considered the effect of mooring lines pre-tensioning.

Faltinsen (1993) presented a mooring system stiffness matrix for a horizontal plane motion. The author also formulated it from the differentiation of forces with respect to generalized coordinates. The matrix is computed for a trivial equilibrium position. However, the author did not take into account the effects of the mooring line pre-tensioning.

Sannasiraj, Sundar and Sundaravadivelu (1998) formulated the mooring stiffness matrix for two-dimensional vertical plane motions, for a two-line mooring system. Figure 
Figure 19 - Sketch of the moored floating body.

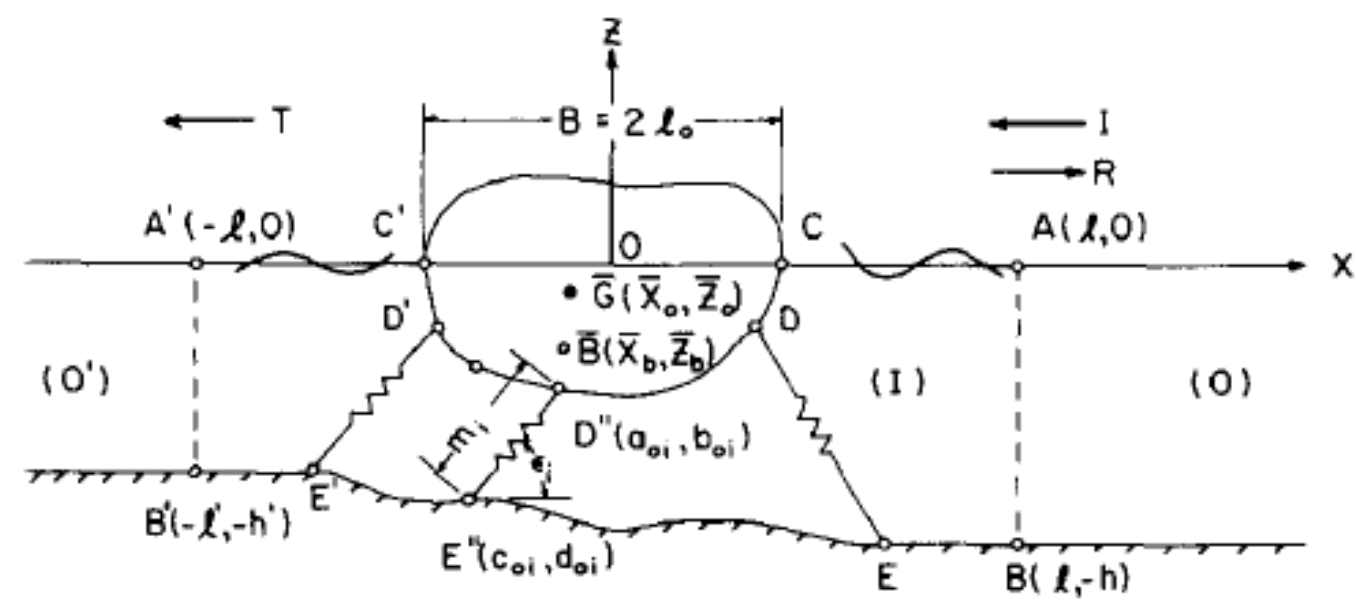

Source: Yamamoto, Yoshida and Ijima (1980)

Figure 20 - Small horizontal displacements and rotations.

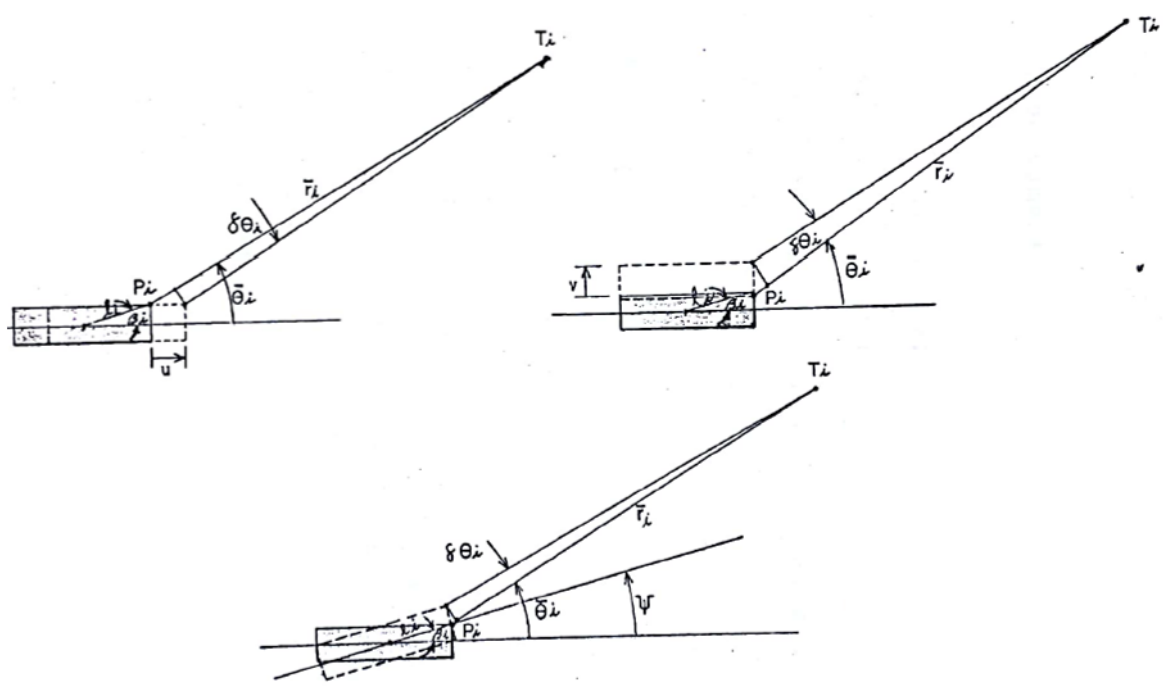

Source: Adapted from Pesce (1986)

21 sketches the problem. Taking advantage of each mooring line tension, the stiffness matrix was calculated from the derivations of them with respects to the coordinates of the problem.

Loukogeorgaki and Angelides (2005) brought an analytical formulation for the $6 \times 6$ mooring system stiffness matrix. Based on perturbation methods, the authors define the coefficients by differentiating forces with respect to generalized coordinates.

Kim et al. (2013) applied prescribed static offset analysis on a FE model. The 
Figure 21 - Vertical plane view of a SMS.

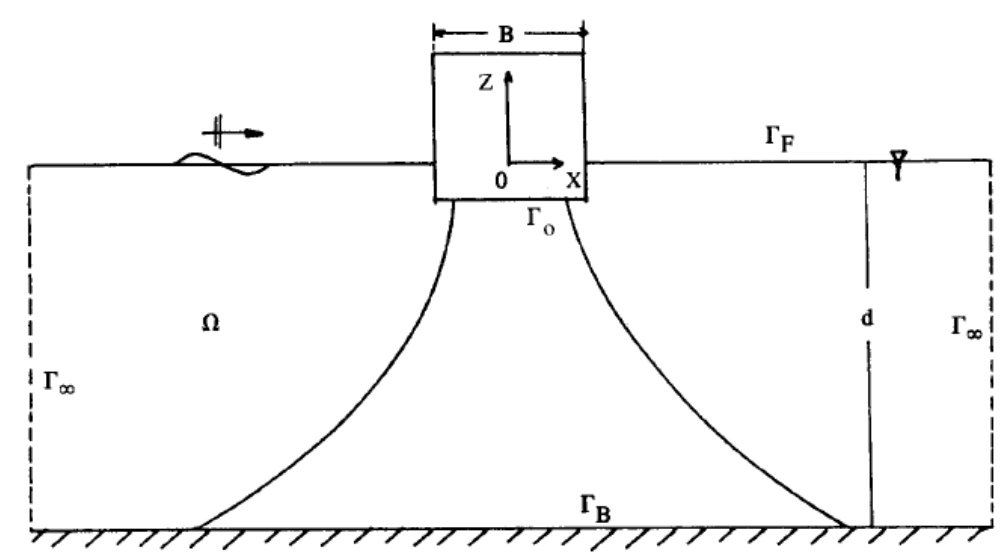

Source: Sannasiraj, Sundar and Sundaravadivelu (1998)

restoring forces due to the mooring system are calculated for a prescribed offset at the vessel. The stiffness coefficients were numerically calculated by the ratio between forces and displacements around the equilibrium position.

In Robertson et al. (2014a), the mooring system stiffness was calculated with a linearized analysis around an equilibrium position of the platform, i.e. the first derivative of each mooring generalized force with respect to generalized directions. This analysis involved a small perturbation in the platform DoFs, followed by measuring the resulting mooring loads, via a numerical computation of forces-displacement curves. The stiffness coefficients were calculated then using central-differences of these curves.

Similarly to Loukogeorgaki and Angelides (2005), Al-Solihat and Nahon (2015) formulated a $6 \times 6$ stiffness matrix from perturbation methods, first for each mooring line and then summing up the effects of all lines. The authors separated the stiffness matrix according to the mooring line configuration. In this context, it is presented final formulations for catenary and taut-leg mooring lines and tension leg systems. Figure 22 brings up one example of mooring system the author used in order to apply their methodology.

Montasir, Yenduri and Kurian (2015) addressed the problem of two-dimensional vertical plane motions. In this context, the authors present a $3 \times 3$ stiffness matrix. It was considered a linear spring coefficient for the horizontal direction and the matrix is defined from the geometry of the problem.

Gutiérrez-Romero et al. (2016) brought up a formulation of the stiffness matrix, but considering translational motions only, i.e. both the two horizontal and the vertical motions. The mooring system was modeled via FE method and force-displacement relations for the system as a whole were computed. The stiffness coefficients were calculated from 
Figure 22 - Six-DoF moored system.

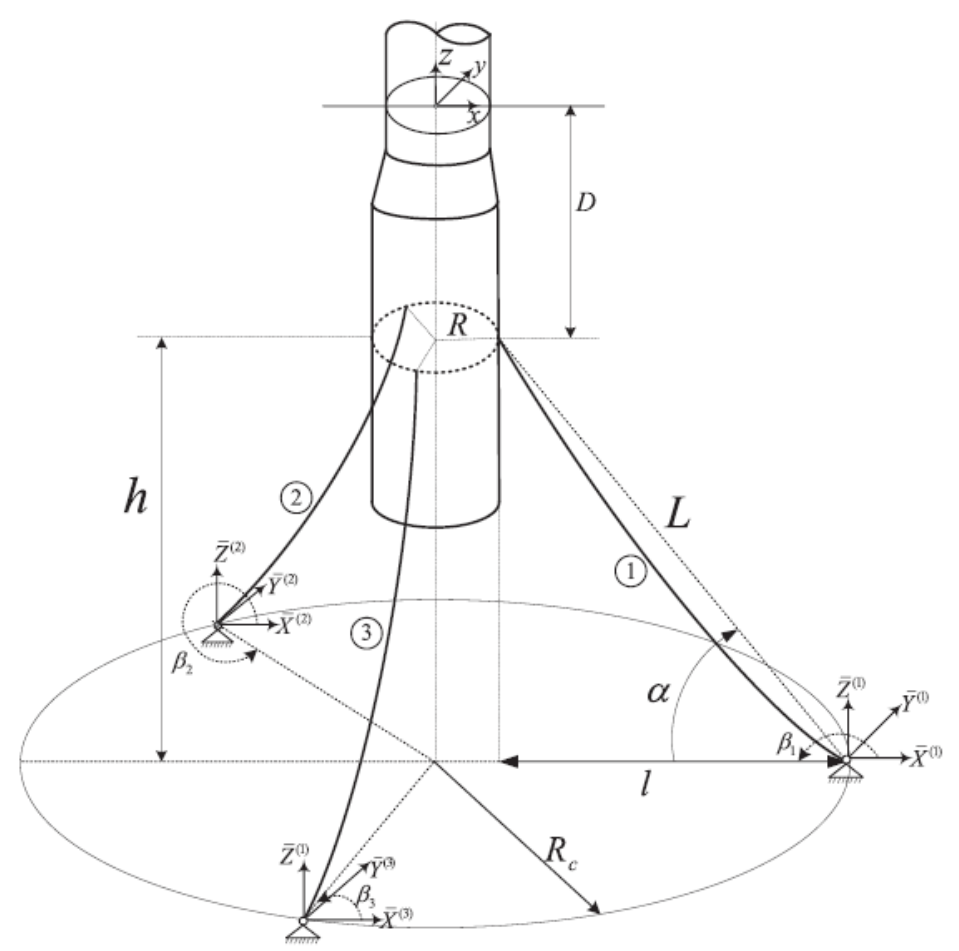

Source: Al-Solihat and Nahon (2015)

the ratio between forces and excursions.

Finally, Pesce, Amaral and Franzini (2018) proposed a $3 \times 3$ stiffness matrix for a horizontal plane problem. Using classical approaches from analytic mechanics, the authors derived the stiffness coefficients in a formal and proper way. For generic mooring line configurations, it was presented a closed formulation for a generic vessel position. A sketch of the investigated problem in Pesce, Amaral and Franzini (2018) is presented in Figure 23.

To better situate the present work, Table 2 summarizes the stiffness matrices found in the literature, presenting which DoF are assessed and the methodology for obtaining them. The present master dissertation brings then, in Section 3.3, an expedite and closed formulation for the six-DoF mooring system stiffness matrix. It is an expansion of the previous work of Pesce, Amaral and Franzini (2018). It differs from Loukogeorgaki and Angelides (2005) and Al-Solihat and Nahon (2015) formulations for the use of Analytical Mechanics, instead of dealing with the perturbation approach. Thus, the matrix is defined in a more formal way. Additionally, generic closed formulation allows the evaluation of the mooring system stiffness at any generic position (not only the trivial equilibrium one) and for any mooring line configuration. Finally, the mooring system stiffness is presented partitioned, proposing an easier way to evaluate all coefficients from the three translational 
Figure 23 - Sketch of a generic moored buoy as a generic position.

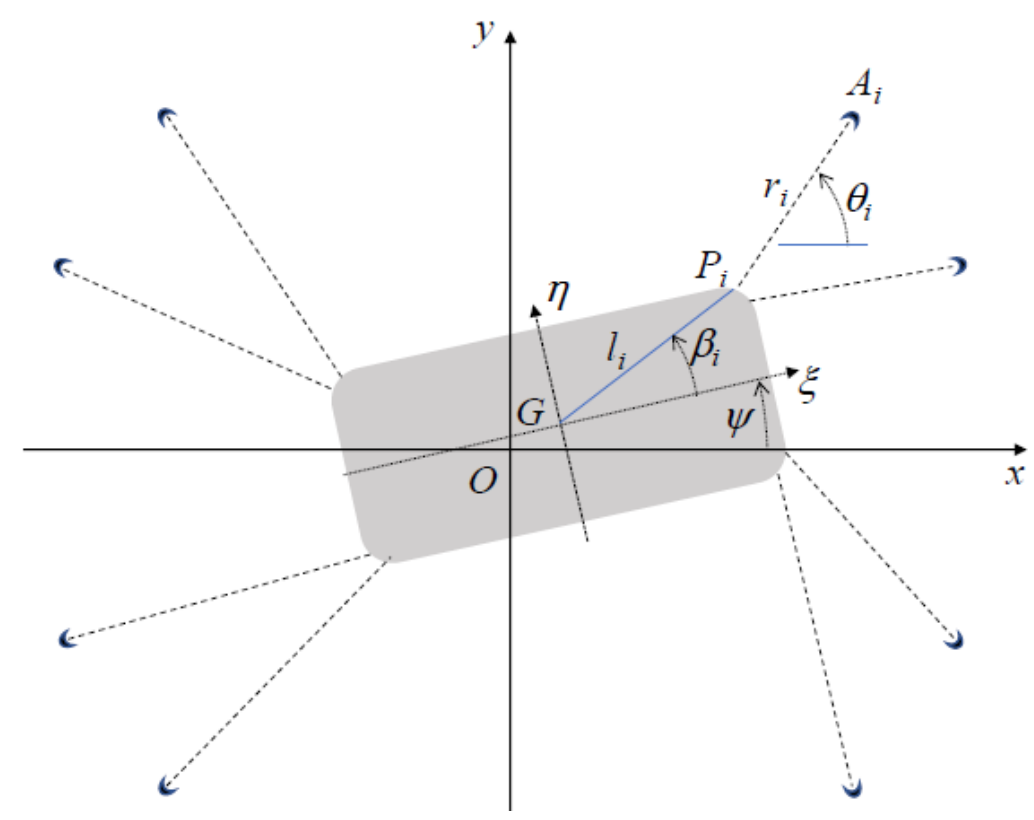

Source: Pesce, Amaral and Franzini (2018)

displacement DoFs (which helps to understand the effects of the vessel's geometry and the position of fairleads on the system's stiffness).

This literature review is closed remarking that the proposed methodology by the present master dissertation are addressed to actual problems on ocean engineering scenario. Some important aspects worthy of investigation can be highlighted: (i) lack of a generic analytic formulation to assess the stiffness of the mooring system, (ii) lack of a tool to investigate effects of the mean vessel position on the stiffness coefficients and natural periods and (iii) lack of understanding the importance of the mooring line axial stiffness on the stiffness. This text aims to fill these gaps. As discussed earlier, an expedite closed formulation for the mooring system stiffness matrix is presented and can be useful not only for the design process but also for the analysis of mooring systems. The methodology herein proposed can help fundamental understanding of the effects of a mooring system parameters on the static (and also dynamic) responses of the moored system. 
Table 2 - Stiffness matrix models presented at the reviewed literature.

\begin{tabular}{lcl}
\hline \hline Authors & DoF & Methodology \\
\hline $\begin{array}{l}\text { Yamamoto, Yoshida and } \\
\text { Ijima (1980) }\end{array}$ & $\begin{array}{c}\text { Vertical Plane } \\
(3 \times 3)\end{array}$ & $\begin{array}{l}\text { Analytical: Perturbation around the } \\
\text { equilibrium position }\end{array}$ \\
\hline Pesce (1986) & $\begin{array}{c}\text { Horizontal Plane } \\
(3 \times 3)\end{array}$ & $\begin{array}{l}\text { Analytical: Perturbation around the } \\
\text { equilibrium position }\end{array}$ \\
\hline Faltinsen (1993) & $\begin{array}{c}\text { Horizontal Plane } \\
(3 \times 3)\end{array}$ & $\begin{array}{l}\text { Analytical: Derivation the forces } \\
\text { with respect to the coordinates }\end{array}$ \\
\hline $\begin{array}{l}\text { Sannasiraj, Sundar and } \\
\text { Sundaravadivelu (1998) }\end{array}$ & $\begin{array}{c}\text { Vertical Plane } \\
(3 \times 3)\end{array}$ & $\begin{array}{l}\text { Analytical: Derivation the forces } \\
\text { with respect to the coordinates }\end{array}$ \\
\hline $\begin{array}{l}\text { Loukogeorgaki and } \\
\text { Angelides (2005) }\end{array}$ & All six $(6 \times 6)$ & $\begin{array}{l}\text { Analytical: Perturbation around the } \\
\text { equilibrium position }\end{array}$ \\
\hline Kim et al. (2013) & All six $(6 \times 6)$ & $\begin{array}{l}\text { Numerical: Finite Elements }- \\
\text { Prescribed static offset analysis }\end{array}$ \\
\hline $\begin{array}{l}\text { Robertson et al. (2014a) } \\
\text { Al-Solihat and Nahon }\end{array}$ & All six $(6 \times 6)$ & $\begin{array}{l}\text { Analytical: Prescribed static offset } \\
\text { analysis }\end{array}$ \\
\hline $\begin{array}{l}\text { Mo15) } \\
\text { Kontasir, Yenduri and }(6 \times 6)\end{array}$ & $\begin{array}{l}\text { Analytical: Perturbation around the } \\
\text { equilibrium position }\end{array}$ \\
\hline $\begin{array}{l}\text { Gutiérrez-Romero et al. } \\
(2016)\end{array}$ & $\begin{array}{c}\text { Translational } \\
(3 \times 3)\end{array}$ & $\begin{array}{l}\text { Analytical: Assumption of the surge } \\
\text { stiffness coefficient }\end{array}$ \\
\hline $\begin{array}{l}\text { Pesce, Amaral and } \\
\text { Franzini (2018) }\end{array}$ & Horizontal Plane \\
$(3 \times 3)$ & Analytical: Analytical Mechanics \\
\hline \hline
\end{tabular}

Source: The Author 



\section{Mathematical Models}

The current chapter presents the mathematical models developed in this work. Firstly, Section 3.1 shows the geometry of the body and the geometric relations for each mooring line. Section 3.2 presents the development of a single mooring line restoring force, and the decomposition of this force into the line's two main directions: horizontal and vertical. In turn, Section 3.3 brings a linearization of the problem around a generic position ${ }^{1}$, in order to define the full mooring system stiffness matrix. The term "position of the floating body" is herein understood in a generic sense, as the formulation treats a six-DoF problem. The term "trivial equilibrium position" is reserved to that for the externally unloaded system, i.e. under no current, wind or wave forces, nor any other external one, as a towing force applied by a tug boat, for instance. If restricted to motions on the horizontal plane, the term "generic position" may be interpreted as "offset" and "heading", regarding the trivial equilibrium one. Finally, Section 3.4 conducts a formulation for a multi-segment mooring line tension as function of horizontal and vertical distances between anchor and fairlead. Concluding, it is important to observe that the present chapter summarizes the main contributions of this work.

\subsection{Geometric Relations of Floating Unit and Mooring Lines}

The current section aims to define geometric relations between a moored body and its mooring lines. It is important to note that, for all the following development, it is not defined any specific type of line configuration. Indeed, the mooring line model will only be defined in Section 3.4, when the multi-segment mooring line is modeled, considered the context of the case studies of Chapter 4.

Initially, it is important to define the generalized coordinates for the problem. Let us consider a floating body on a generic position, moored by $N$ mooring lines as sketched in Figure 24. Let us $O x y z$ be an inertial reference frame, grounded to Earth. Its origin $O$ is defined at the center of mass of the body at equilibrium position of the autonomous (or unloaded) system. This frame is defined by the orthonormal basis $E_{x}=\left(\hat{e}_{x}, \hat{e}_{y}, \hat{e}_{z}\right)$. In addition, let us $G \xi \eta \zeta$ be an orthonormal moving frame fixed to the body. The origin of this frame is for convenience taken as the center of mass of the body, without losing generalization. The basis that defines this frame is $E_{\xi}=\left(\hat{e}_{\xi}, \hat{e}_{\eta}, \hat{e}_{\zeta}\right)$.

In order to define the relation between the two reference frames, one can define the

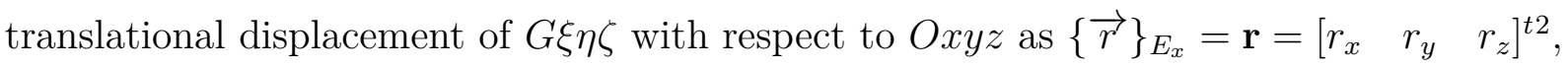

1 The mooring system stiffness matrix is formulated for any given position.

2 For this master dissertation, the notation $\{\rightarrow\}_{E_{x}}$ denotes a physical vector while the letter in bold 
Figure 24 - Sketch of a generic floating body, moored by N mooring lines at: the equilibrium position of the autonomous system (dashed) and a generic displaced position (full lines).

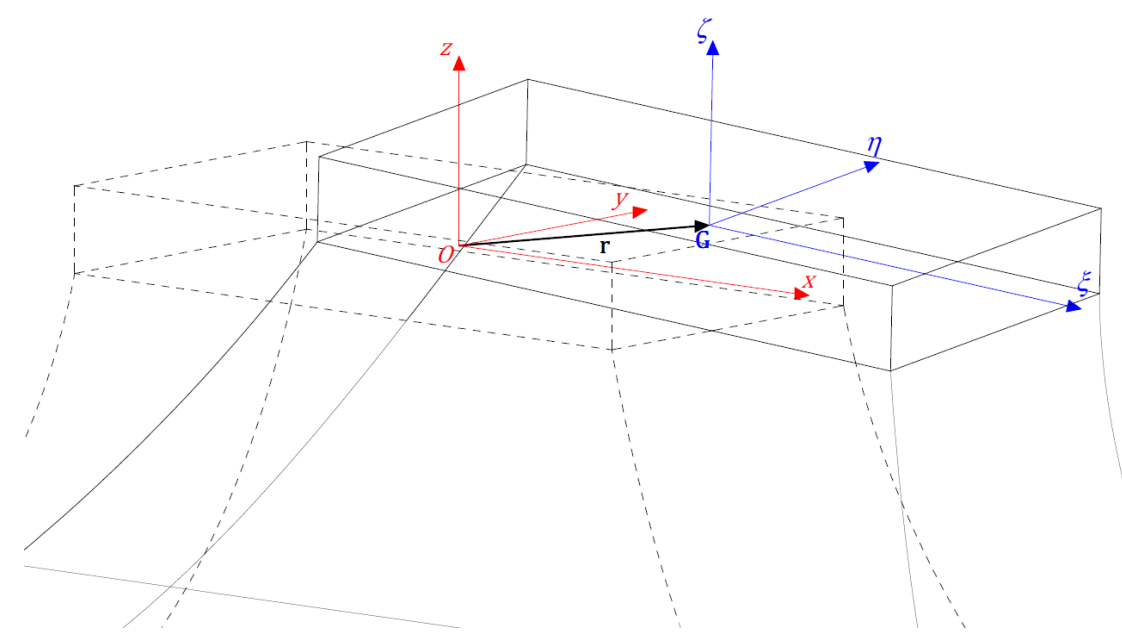

Source: The Author

as presented in the Figure 25. Notice that, as the components of $\mathbf{r}$ are written with respect to the fixed reference frame, one should not mistake these displacements with classical Ocean Engineering surge, sway and heave motions, all defined on the body reference frame. The rotation with respect to the reference frames is defined by the Euler's

Figure 25 - Translational displacement between $G \xi \eta \zeta$ and $O x y z$.

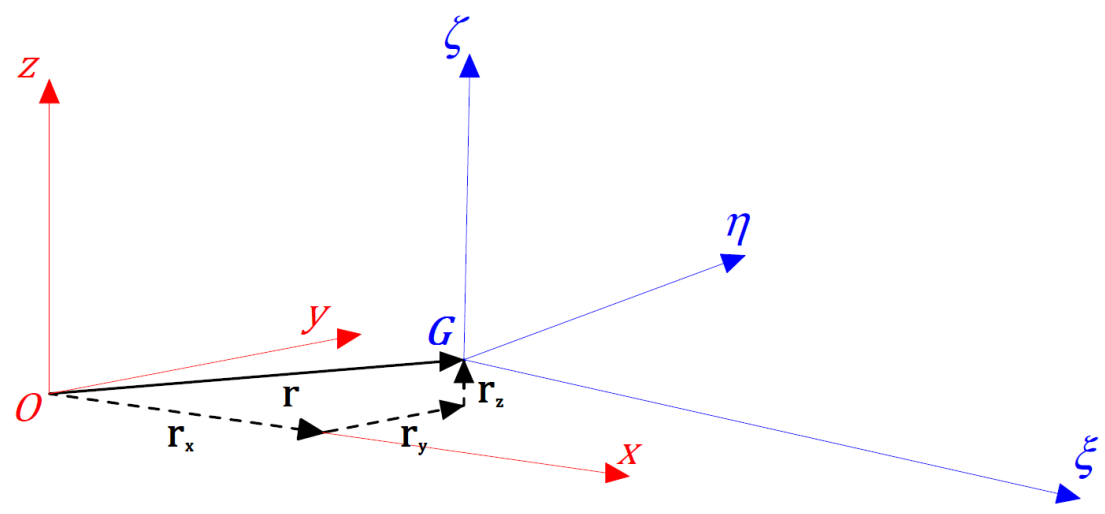

Source: The Author

angles $\boldsymbol{\theta}=\left[\begin{array}{lll}\phi & \theta & \psi\end{array}\right]^{t}$, as shown in Figure 26. In other words, the rotation angles of the floating body are defined following the Ocean Engineering order: $\psi$ is defined as the rotation angle around the current $\zeta$ axis, then $\theta$ is defined around the current $\eta$ axis, and finally $\phi$ is the rotation angle around the current $\xi$ axis. Then, it is possible to define

represents a first order tensor. Notice that the translational displacement can be defined in both notations. 
$\mathbf{q}=\left[\begin{array}{llllll}r_{x} & r_{y} & r_{z} & \phi & \theta & \psi\end{array}\right]^{t}$ as the generalized coordinate vector defining the floating unit motions.

Figure 26 - Definition of the Euler Angles.

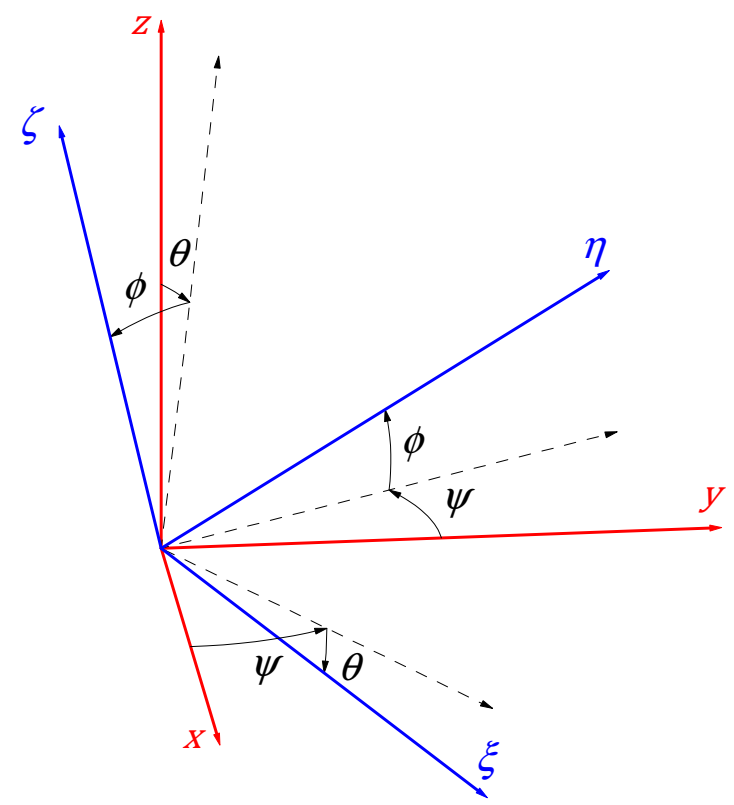

Source: The Author

Figure 27 - Generic floating body, moored by N mooring lines at a generic position.

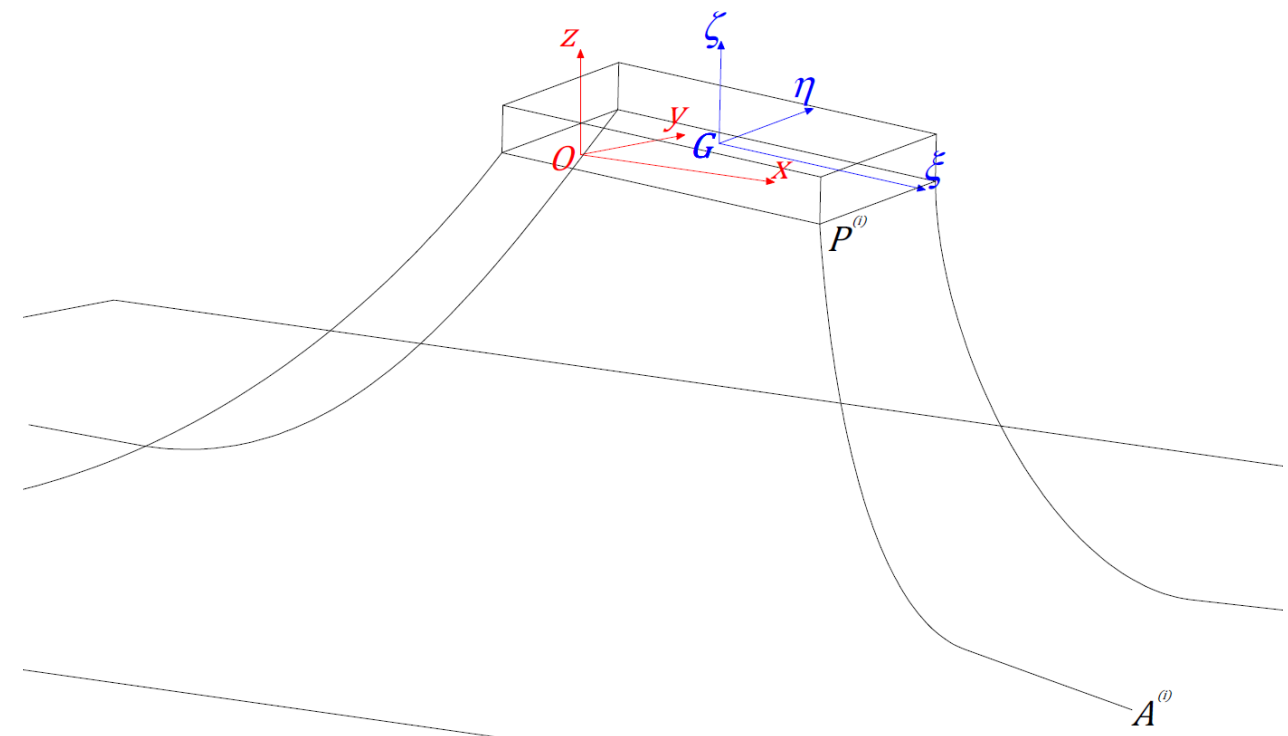

Source: The Author

Now, focus is on defining the position of each mooring line fairlead $P^{(i) 3}$ and anchor $\left.\overline{\text { Throughout the text, the superscript }}^{(}{ }^{i}\right)$ refers to the $i$-th mooring line. 
$A^{(i)}$ with respect to the fixed frame. Figure 27 brings the N-moored body at the displaced position.

One can write $P^{(i)}$ with respect to the moving frame as:

$$
\left\{\vec{P}^{(i)}\right\}_{E_{\xi}}=\left(P^{(i)}-G\right)=\left[\begin{array}{lll}
p_{\xi}^{(i)} & p_{\eta}^{(i)} & p_{\zeta}^{(i)}
\end{array}\right]^{t}
$$

Then, considering the Euler Angles defined in Figure 26, one can write the rotation matrix $[\mathbb{R}]_{E_{x} \mid E_{\xi}}$ as follows:

$$
[\mathbb{R}]_{E_{x} \mid E_{\xi}}=\left(\begin{array}{ccc}
\cos \theta \cos \psi & \sin \phi \sin \theta \cos \psi-\cos \phi \sin \psi & \cos \phi \sin \theta \cos \psi+\sin \phi \sin \psi \\
\cos \theta \sin \psi & \sin \phi \sin \theta \sin \psi+\cos \phi \cos \psi & \cos \phi \sin \theta \sin \psi-\sin \phi \cos \psi \\
-\sin \theta & \sin \phi \cos \theta & \cos \phi \cos \theta
\end{array}\right)
$$

By composing the translational and rotational movements, it is possible to write $\left\{\vec{P}^{(i)}\right\}_{E_{x}}$, as follows:

$$
\left\{\vec{P}^{(i)}\right\}_{E_{x}}=\left(P^{(i)}-O\right)=\left[\begin{array}{lll}
p_{x}^{(i)} & p_{y}^{(i)} & p_{z}^{(i)}
\end{array}\right]^{t}=\{\vec{r}\}_{E_{x}}+[\mathbb{R}]_{E_{x} \mid E_{\xi}}\left\{\vec{P}^{(i)}\right\}_{E_{\xi}}
$$

In turns, from Figure 27, one can define the position of each mooring line anchor at the grounded reference frame:

$$
\left\{\vec{A}^{(i)}\right\}_{E_{x}}=\left(A^{(i)}-O\right)=\left[\begin{array}{lll}
a_{x}^{(i)} & a_{y}^{(i)} & a_{z}^{(i)}
\end{array}\right]^{t}
$$

In this context, the horizontal and vertical distances between anchor and fairlead $\left(h^{(i)}\right.$ and $v^{(i)}$, respectively) are determined as:

$$
\begin{aligned}
& h^{(i)}=\sqrt{\left(a_{x}^{(i)}-p_{x}^{(i)}\right)^{2}+\left(a_{y}^{(i)}-p_{y}^{(i)}\right)^{2}} \\
& v^{(i)}=p_{z}^{(i)}-a_{z}^{(i)}
\end{aligned}
$$

These two distances are associated with two directional vectors that defines the fixed reference frame, $\left\{\hat{e}_{h}^{(i)}\right\}_{E_{x}}$ and $\left\{\hat{e}_{v}^{(i)}\right\}_{E_{x}}$. In order to define them, it is important to first define the projection of both fairlead and anchor into the horizontal and vertical planes. Firstly, let us define the horizontal plane $\pi_{h}$ as the one characterized by the unit vectors $\left(\hat{e}_{x}, \hat{e}_{y}\right)$, containing the fairlead $P^{(i)}$.

The projection of $\left\{\vec{A}^{(i)}\right\}_{E_{x}}$ onto $\pi_{h}$ is:

$$
\left\{\vec{A}^{(i)}\right\}_{E_{x}}^{\pi_{h}}=\operatorname{proj}_{\pi_{h}}\left\{\vec{A}^{(i)}\right\}_{E_{x}}=a_{x}^{(i)}\left\{\hat{e}_{x}\right\}_{E_{x}}+a_{y}^{(i)}\left\{\hat{e}_{y}\right\}_{E_{x}}
$$

Thus, it is possible to define the horizontal directional unit vector, for each mooring line as following:

$$
\left\{\hat{e}_{h}^{(i)}\right\}_{E_{x}}=\frac{\left(\left\{\vec{A}^{(i)}\right\}_{E_{x}}^{\pi_{h}}-\left\{\vec{P}^{(i)}\right\}_{E_{x}}\right)}{\left|\left\{\vec{A}^{(i)}\right\}_{E_{x}}^{\pi_{h}}-\left\{\vec{P}^{(i)}\right\}_{E_{x}}\right|}=\cos \alpha^{(i)} \hat{e}_{x}+\sin \alpha^{(i)} \hat{e}_{y}
$$


where:

$$
\begin{aligned}
\cos \alpha^{(i)} & =\frac{a_{x}^{(i)}-p_{x}^{(i)}}{h^{(i)}} \\
\sin \alpha^{(i)} & =\frac{a_{y}^{(i)}-p_{y}^{(i)}}{h^{(i)}}
\end{aligned}
$$

In turn, the vertical plane $\pi_{v}$ is perpendicular to $\pi_{h}$ plane which normal is $\left\{\hat{e}_{h}^{(i)}\right\}_{E_{x}}$, also containing the fairlead $P^{(i)}$. The projection of $\left\{\vec{A}^{(i)}\right\}_{E_{x}}$ onto $\pi_{v}$ is as follows:

$$
\left\{\vec{A}^{(i)}\right\}_{E_{x}}^{\pi_{v}}=\operatorname{proj}_{\pi_{v}}\left\{\vec{A}^{(i)}\right\}_{E_{x}}=a_{z}^{(i)}\left\{\hat{e}_{z}\right\}_{E_{x}}
$$

Then, the vertical directional unit vector for each mooring line becomes:

$$
\left\{\hat{e}_{v}^{(i)}\right\}_{E_{x}}=\frac{\left(\left\{\vec{A}^{(i)}\right\}_{E_{x}}^{\pi_{v}}-\left\{\vec{P}^{(i)}\right\}_{E_{x}}\right)}{\left|\left\{\vec{A}^{(i)}\right\}_{E_{x}}^{\pi_{v}}-\left\{\vec{P}^{(i)}\right\}_{E_{x}}\right|}=\frac{a_{z}^{(i)}-p_{z}^{(i)}}{v^{(i)}} \hat{e}_{z}=-\hat{e}_{z}
$$

Figure 28 illustrates these two planes and the directional unit vectors.

Figure 28 - Mooring line planes and directional unit vectors.

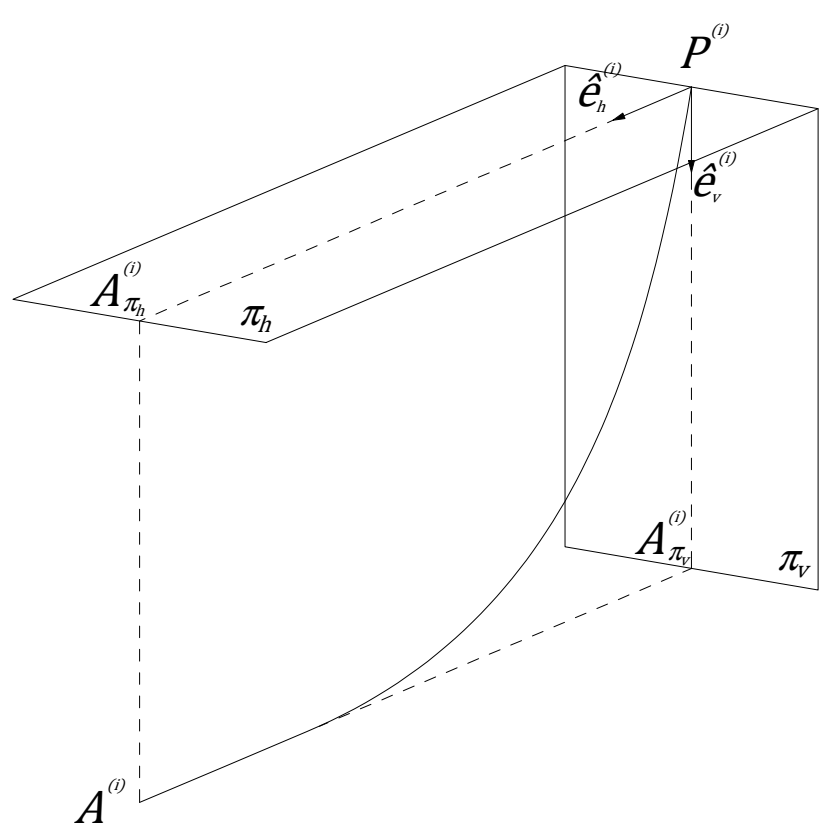

Source: The Author

Finally, it is important to notice that $h^{(i)}, v^{(i)},\left\{\hat{e}_{v}^{(i)}\right\}_{E_{x}},\left\{\hat{e}_{v}^{(i)}\right\}_{E_{x}}$ and $\alpha^{(i)}$ are functions of the generalized coordinates, since they depend on the position of the fairlead with respect to the fixed frame, $\left\{\vec{P}^{(i)}\right\}_{E_{x}}$.

From the next section onward, as a matter of practicality, vectors' basis are omitted whenever there is no doubt which reference frame is used. For example, the two directional 
vectors are represented now on as $\hat{e}_{h}^{(i)}$ and $\hat{e}_{v}^{(i)}$, since they are always expressed on the fixed reference frame.

Next section brings forward the relation between the aforementioned geometric quantities and the forces each mooring line imposes on the floating unit.

\subsection{Mooring System Restoring Forces}

Figure 29 presents the force that a mooring line imposes on the vessel a the i-th fairlead. It also shows the decomposition of this force into the vertical and horizontal directions. Notice that the horizontal and vertical components are referred to the directional unit vectors defined in Equations 3.8 and 3.12.

Figure 29 - i-th mooring line tension at the fairlead.

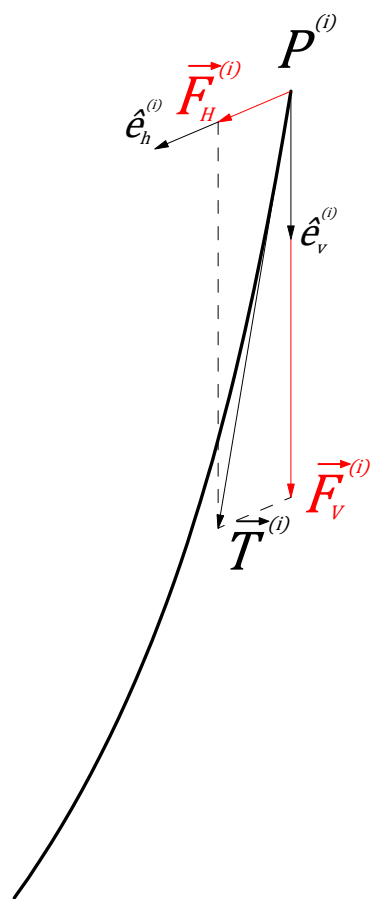

Source: The Author

A strong but justified hypothesis is taken into account: one may neglect any possible effect of friction with the seabed. Additionally, the effect of sea current on the mooring line is not considered. Therefore, the mooring lines are supposed to be subjected only to gravity and to elastic effects due to extensibility of the line, besides reaction forces applied to their extremities, fairleads and anchors, here considered as holonomic constraints. In summary, the system is considered conservative, mooring lines remaining in vertical planes. It is also worth mentioning that the present model does not consider the dynamics of the mooring lines and may be considered quasi-static in this sense. These hypotheses guarantee that 
the direction of the tension at the fairlead remains at the plane of the line, as presented in the following developments. Thus, the tension can be written as function of $h^{(i)}$ and $v^{(i)}$. This relation can be called the characteristic tension function of the mooring line. Equation 3.13 recovers this discussion.

$$
\vec{T}^{(i)}\left(h^{(i)}, v^{(i)}\right)=F_{H}^{(i)}\left(h^{(i)}, v^{(i)}\right) \hat{e}_{h}^{(i)}+F_{V}^{(i)}\left(r^{(i)}, h^{(i)}\right) \hat{e}_{v}^{(i)}
$$

It is noteworthy that the development made until this point is general, i.e. it is not restrict to a single type of mooring line configuration. However, the functions $F_{H}^{(i)}$ and $F_{V}^{(i)}$ should be known a priori. The way to obtain these curves may vary. On the one hand, for a simple catenary laying curve, analytical formulations are well-known. On the other hand, for more sophisticated mooring line arrangements, it might be necessary to evaluate them numerically (e.g. by finite elements method). An example of analytical formulations for multi-segment mooring line is presented in Section 3.4. Once again, notice that since $F_{H}^{(i)}$ and $F_{V}^{(i)}$ are functions of $h^{(i)}$ and $v^{(i)}$, they are consequently functions of $\mathbf{q}$.

Considering the effect of every mooring line, the restoring generalized force vector, $\mathrm{Q}$ can be defined with respect to each generalized coordinate $q_{j}, j=1, \ldots, 6$.

$$
\mathbf{Q}=\left[\begin{array}{llllll}
Q_{r_{x}} & Q_{r_{y}} & Q_{r_{z}} & Q_{\phi} & Q_{\theta} & Q_{\psi}
\end{array}\right]^{t}
$$

where, from Analytical Mechanics:

$$
Q_{j}=\sum_{i=1}^{N} Q_{j}^{(i)}=\sum_{i=1}^{N} \overrightarrow{T^{(i)}} \cdot \frac{\partial \vec{P}^{(i)}}{\partial q_{j}}=\sum_{i=1}^{N}\left(F_{H}^{(i)} \hat{e}_{h}^{(i)}+F_{V}^{(i)} \hat{e}_{v}^{(i)}\right) \cdot \frac{\partial \vec{P}^{(i)}}{\partial q_{j}}
$$

where $Q_{j}^{(i)}$ is the generalized restoring force associated with the $i$-th line.

In order to evaluate the generalized restoring forces, it is needed to develop local generalized directions $\partial \vec{P}^{(i)} / \partial q_{j}$ and their projections onto the horizontal and vertical planes. These developments are presented in Appendix C.

Hence, it is possible to write the generalized restoring forces from each mooring line as follows:

$$
\begin{aligned}
& Q_{r_{x}}^{(i)}=F_{H}^{(i)} \cos \alpha^{(i)} \\
& Q_{r_{y}}^{(i)}=F_{H}^{(i)} \sin \alpha^{(i)} \\
& Q_{r_{z}}^{(i)}=-F_{V}^{(i)} \\
& Q_{\phi}^{(i)}=F_{H}^{(i)}\left(\cos \alpha^{(i)} \frac{\partial p_{x}^{(i)}}{\partial \phi}+\sin \alpha^{(i)} \frac{\partial p_{y}^{(i)}}{\partial \phi}\right)-F_{V}^{(i)} \frac{\partial p_{z}^{(i)}}{\partial \phi} \\
& Q_{\theta}^{(i)}=F_{H}^{(i)}\left(\cos \alpha^{(i)} \frac{\partial p_{x}^{(i)}}{\partial \theta}+\sin \alpha^{(i)} \frac{\partial p_{y}^{(i)}}{\partial \theta}\right)-F_{V}^{(i)} \frac{\partial p_{z}^{(i)}}{\partial \theta} \\
& Q_{\psi}^{(i)}=F_{H}^{(i)}\left(\cos \alpha^{(i)} \frac{\partial p_{x}^{(i)}}{\partial \psi}+\sin \alpha^{(i)} \frac{\partial p_{y}^{(i)}}{\partial \psi}\right)
\end{aligned}
$$


Notice that with an easy algebraic manipulation it is possible to write $Q_{\phi}^{(i)}, Q_{\theta}^{(i)}$ and $Q_{\psi}^{(i)}$ as combinations of $Q_{r_{x}}^{(i)}, Q_{r_{y}}^{(i)}$ and $Q_{r_{z}}^{(i)}$. The restoring moments become:

$$
\begin{aligned}
Q_{\phi}^{(i)} & =Q_{r_{x}}^{(i)} \frac{\partial p_{x}}{\partial \phi}+Q_{r_{y}}^{(i)} \frac{\partial p_{y}}{\partial \phi}+Q_{r_{z}}^{(i)} \frac{\partial p_{z}}{\partial \phi} \\
Q_{\theta}^{(i)} & =Q_{r_{x}}^{(i)} \frac{\partial p_{x}}{\partial \theta}+Q_{r_{y}}^{(i)} \frac{\partial p_{y}}{\partial \theta}+Q_{r_{z}}^{(i)} \frac{\partial p_{z}}{\partial \theta} \\
Q_{\psi}^{(i)} & =Q_{r_{x}}^{(i)} \frac{\partial p_{x}}{\partial \psi}+Q_{r_{y}}^{(i)} \frac{\partial p_{y}}{\partial \psi}
\end{aligned}
$$

The definition of the generalized restoring forces made in Equations 3.16 to 3.18 and 3.22 to 3.24 are crucial for the following steps of this master thesis. Next section brings forward a linearization of these forces around a supposed known equilibrium position in order to define the mooring system stiffness matrix.

\subsection{Mooring System Stiffness Matrix}

The hypothesis of neglecting friction effects, adopted for describing the mooring line restoring forces as functions of the position only, allows us to see them as conservative forces. In this case, the generalized forces can be seen as potential forces, i.e. forces arisen from a potential functional. From Analytical Mechanics, one can write:

$$
Q_{j}=-\frac{\partial V}{\partial q_{j}}
$$

where $V=V(\mathbf{q}, \Pi)$ is the potential energy and $\Pi=\left\{\left(A^{(i)}, P^{(i)}, F_{H}^{(i)}, F_{V}^{(i)}\right) \mid i=1, \ldots, N\right\}$ is the set of geometric parameters of the mooring system and vessel.

Then, locally, around any pre-stated equilibrium configuration, the mooring system stiffness matrix $\mathbb{K}(\mathbf{q})$ may be defined as the Hessian of $V(\mathbf{q}, \Pi)$ :

$$
\mathbb{K}(\mathbf{q})=\left[\frac{\partial^{2} V}{\partial q_{j} \partial q_{k}}\right]=-\left[\frac{\partial Q_{j}}{\partial q_{k}}\right]
$$

Generically, from Equation 3.15, follows that:

$$
\begin{aligned}
& K_{j k}=-\frac{\partial Q_{j}}{\partial q_{k}}=-\frac{\partial}{\partial q_{k}}\left(\sum_{i=1}^{N} \vec{T}^{(i)} \cdot \frac{\partial \vec{P}^{(i)}}{\partial q_{j}}\right)= \\
&-\sum_{i=1}^{N} \frac{\partial}{\partial q_{k}}\left[\left(F_{H}^{(i)} \hat{e}_{h}^{(i)}+F_{V}^{(i)} \hat{e}_{v}^{(i)}\right) \cdot \frac{\partial \vec{P}^{(i)}}{\partial q_{j}}\right]
\end{aligned}
$$


Recalling that $F_{H}^{(i)}=F_{H}^{(i)}\left(h^{(i)}, v^{(i)}\right)$ and $F_{H}^{(i)}=F_{H}^{(i)}\left(h^{(i)}, v^{(i)}\right)$, Equation 3.27 becomes:

$$
\begin{aligned}
& K_{j k}=\sum_{i=1}^{N} K_{j k}^{(i)}=-\sum_{i=1}^{N}\left(\frac{\partial F_{H}^{(i)}}{\partial h^{(i)}} \frac{\partial h^{(i)}}{\partial q_{k}}+\frac{\partial F_{H}^{(i)}}{\partial v^{(i)}} \frac{\partial v^{(i)}}{\partial q_{k}}\right) \hat{e}_{h}^{(i)} \cdot \frac{\partial \vec{P}^{(i)}}{\partial q_{j}}+ \\
&-\sum_{i=1}^{N}\left(\frac{\partial F_{V}^{(i)}}{\partial h^{(i)}} \frac{\partial h^{(i)}}{\partial q_{k}}+\frac{\partial F_{V}^{(i)}}{\partial v^{(i)}} \frac{\partial v^{(i)}}{\partial q_{k}}\right) \hat{e}_{v}^{(i)} \cdot \frac{\partial \vec{P}^{(i)}}{\partial q_{j}}+ \\
&-\sum_{i=1}^{N}\left[F_{H}^{(i)} \frac{\partial}{\partial q_{k}}\left(\hat{e}_{h}^{(i)} \cdot \frac{\partial \vec{P}^{(i)}}{\partial q_{j}}\right)+F_{V}^{(i)} \frac{\partial}{\partial q_{k}}\left(\hat{e}_{v}^{(i)} \cdot \frac{\partial \vec{P}^{(i)}}{\partial q_{j}}\right)\right]
\end{aligned}
$$

with $K_{j k}^{(i)}$ being the stiffness coefficient associated with the i-th mooring line.

In order to simplify the notation, it is possible to identify the terms $\partial F_{H}^{(i)} / \partial h^{(i)}=$ $k_{H H}$ and $\partial F_{V}^{(i)} / \partial v^{(i)}=k_{V V}$ as the local horizontal and vertical stiffness, respectively. It can be defined two coupled local stiffness as well: $\partial F_{H}^{(i)} / \partial v^{(i)}=k_{H V}$ and $\partial F_{V}^{(i)} / \partial h^{(i)}=k_{V H}$. It can be shown that the two coupled terms are equal. Appendix A shows this demonstration for a multi-segment mooring line.

Then, for each mooring line:

$$
\begin{aligned}
K_{j k}^{(i)}=-\left(k_{H H}^{(i)} \frac{\partial h^{(i)}}{\partial q_{k}}+k_{H V}^{(i)} \frac{\partial v^{(i)}}{\partial q_{k}}\right) \hat{e}_{h}^{(i)} \cdot \frac{\partial \vec{P}^{(i)}}{\partial q_{j}}-F_{H}^{(i)} \frac{\partial}{\partial q_{k}}\left(\hat{e}_{h}^{(i)} \cdot \frac{\partial \vec{P}^{(i)}}{\partial q_{j}}\right) \\
-\left(k_{V H}^{(i)} \frac{\partial h^{(i)}}{\partial q_{k}}+k_{V V}^{(i)} \frac{\partial v^{(i)}}{\partial q_{k}}\right) \hat{e}_{v}^{(i)} \cdot \frac{\partial \vec{P}^{(i)}}{\partial q_{j}}-F_{V}^{(i)} \frac{\partial}{\partial q_{k}}\left(\hat{e}_{v}^{(i)} \cdot \frac{\partial \vec{P}^{(i)}}{\partial q_{j}}\right)
\end{aligned}
$$

For the sake of the text's conciseness, the intermediate developments such as the derivative of $\hat{e}_{h}^{(i)}$ and $\hat{e}_{v}^{(i)}$ are left for Appendix D. Finally, it is possible to write the stiffness coefficients due to the mooring system. Before this, it is still necessary to define another stiffness term $\bar{k}_{H H}\left(h^{(i)}\right)=F_{H}^{(i)} / h^{(i)}$, as the "string stiffness" associated with the pre-tensioning of each mooring line as function of $\mathbf{q}$. This "string stiffnes" was introduced by Pesce, Amaral and Franzini (2018). The authors state that its effect is crucial, mainly for some symmetric mooring arrangements. They have presented an example using the OC4 semi-submersible to illustrate its importance. In the present work, the same case study is taken into account in Section 4 , where the effect of $\bar{k}_{H H}\left(r^{(i)}\right)$ is once again highlighted.

Thereafter, it is possible to rewrite the stiffness coefficients given by Equation 3.29, as follows: 
- First line coefficients - coefficients associated with a displacement $r_{x}$ :

$$
\begin{aligned}
K_{11}^{(i)} & =k_{H H}^{(i)} \cos ^{2} \alpha^{(i)}+\bar{k}_{H H}^{(i)} \sin ^{2} \alpha^{(i)} \\
K_{12}^{(i)} & =\left(k_{H H}^{(i)}-\bar{k}_{H H}^{(i)}\right) \cos \alpha^{(i)} \sin \alpha^{(i)} \\
K_{13}^{(i)} & =-k_{H V}^{(i)} \cos \alpha^{(i)} \\
K_{14}^{(i)} & =\frac{\partial p_{x}^{(i)}}{\partial \phi} K_{11}^{(i)}+\frac{\partial p_{y}^{(i)}}{\partial \phi} K_{12}^{(i)}+\frac{\partial p_{z}^{(i)}}{\partial \phi} K_{13}^{(i)} \\
K_{15}^{(i)} & =\frac{\partial p_{x}^{(i)}}{\partial \theta} K_{11}^{(i)}+\frac{\partial p_{y}^{(i)}}{\partial \theta} K_{12}^{(i)}+\frac{\partial p_{z}^{(i)}}{\partial \theta} K_{13}^{(i)} \\
K_{16}^{(i)} & =\frac{\partial p_{x}^{(i)}}{\partial \psi} K_{11}^{(i)}+\frac{\partial p_{y}^{(i)}}{\partial \psi} K_{12}^{(i)}
\end{aligned}
$$

- Second line coefficients - coefficients associated with a displacement $r_{y}$ :

$$
\begin{aligned}
& K_{21}^{(i)}=\sum_{i=1}^{N}\left(k_{H H}^{(i)}-\bar{k}_{H H}^{(i)}\right) \sin \alpha^{(i)} \cos \alpha^{(i)} \\
& K_{22}^{(i)}=k_{H H}^{(i)} \sin ^{2} \alpha^{(i)}+\bar{k}_{H H}^{(i)} \cos ^{2} \alpha^{(i)}
\end{aligned}
$$

$$
\begin{aligned}
& K_{23}^{(i)}=-k_{H V}^{(i)} \sin \alpha^{(i)} \\
& K_{24}^{(i)}=\frac{\partial p_{x}^{(i)}}{\partial \phi} K_{21}^{(i)}+\frac{\partial p_{y}^{(i)}}{\partial \phi} K_{22}^{(i)}+\frac{\partial p_{z}^{(i)}}{\partial \phi} K_{23}^{(i)} \\
& K_{25}^{(i)}=\frac{\partial p_{x}^{(i)}}{\partial \theta} K_{21}^{(i)}+\frac{\partial p_{y}^{(i)}}{\partial \theta} K_{22}^{(i)}+\frac{\partial p_{z}^{(i)}}{\partial \theta} K_{23}^{(i)} \\
& K_{26}^{(i)}=\frac{\partial p_{x}^{(i)}}{\partial \psi} K_{21}^{(i)}+\frac{\partial p_{y}^{(i)}}{\partial \psi} K_{22}^{(i)}
\end{aligned}
$$

- Third line coefficients - coefficients associated with a displacement $r_{z}$ :

$$
\begin{aligned}
K_{31}^{(i)} & =-k_{V H}^{(i)} \cos \alpha^{(i)} \\
K_{32}^{(i)} & =-k_{V H}^{(i)} \sin \alpha^{(i)} \\
K_{33}^{(i)} & =k_{V V}^{(i)} \\
K_{34}^{(i)} & =\frac{\partial p_{x}^{(i)}}{\partial \phi} K_{31}^{(i)}+\frac{\partial p_{y}^{(i)}}{\partial \phi} K_{32}^{(i)}+\frac{\partial p_{z}^{(i)}}{\partial \phi} K_{33}^{(i)} \\
K_{35}^{(i)} & =\frac{\partial p_{x}^{(i)}}{\partial \theta} K_{31}^{(i)}+\frac{\partial p_{y}^{(i)}}{\partial \theta} K_{32}^{(i)}+\frac{\partial p_{z}^{(i)}}{\partial \theta} K_{33}^{(i)} \\
K_{36}^{(i)} & =\frac{\partial p_{x}^{(i)}}{\partial \psi} K_{31}^{(i)}+\frac{\partial p_{y}^{(i)}}{\partial \psi} K_{32}^{(i)}
\end{aligned}
$$


- Fourth line coefficients - coefficients associated with a displacement $\phi$ :

$$
\begin{aligned}
K_{41}^{(i)}= & \frac{\partial p_{x}^{(i)}}{\partial \phi} K_{11}^{(i)}+\frac{\partial p_{y}^{(i)}}{\partial \phi} K_{12}^{(i)}+\frac{\partial p_{z}^{(i)}}{\partial \phi} K_{13}^{(i)} \\
K_{42}^{(i)}= & \frac{\partial p_{x}^{(i)}}{\partial \phi} K_{21}^{(i)}+\frac{\partial p_{y}^{(i)}}{\partial \phi} K_{22}^{(i)}+\frac{\partial p_{z}^{(i)}}{\partial \phi} K_{23}^{(i)} \\
K_{43}^{(i)}= & \frac{\partial p_{x}^{(i)}}{\partial \phi} K_{31}^{(i)}+\frac{\partial p_{y}^{(i)}}{\partial \phi} K_{32}^{(i)}+\frac{\partial p_{z}^{(i)}}{\partial \phi} K_{33}^{(i)} \\
K_{44}^{(i)}= & \frac{\partial p_{x}^{(i)}}{\partial \phi} K_{14}^{(i)}+\frac{\partial p_{y}^{(i)}}{\partial \phi} K_{24}^{(i)}+\frac{\partial p_{z}^{(i)}}{\partial \phi} K_{34}^{(i)} \\
\quad-\frac{\partial^{2} p_{x}^{(i)}}{\partial \phi^{2}} F_{H}^{(i)} \cos \alpha^{(i)}-\frac{\partial^{2} p_{y}^{(i)}}{\partial \phi^{2}} F_{H}^{(i)} \sin \alpha^{(i)}+\frac{\partial^{2} p_{z}^{(i)}}{\partial \phi^{2}} F_{V}^{(i)} & \quad-\frac{\partial^{2} p_{x}^{(i)}}{\partial \phi \partial \theta} F_{H}^{(i)} \cos \alpha^{(i)}-\frac{\partial^{2} p_{y}^{(i)}}{\partial \phi \partial \theta} F_{H}^{(i)} \sin \alpha^{(i)}+\frac{\partial^{2} p_{z}^{(i)}}{\partial \phi \partial \theta} F_{V}^{(i)} \\
K_{45}^{(i)}= & \frac{\partial p_{x}^{(i)}}{\partial \phi} K_{15}^{(i)}+\frac{\partial p_{y}^{(i)}}{\partial \phi} K_{25}^{(i)}+\frac{\partial p_{z}^{(i)}}{\partial \phi} K_{35}^{(i)} \\
K_{46}^{(i)}= & \frac{\partial p_{x}^{(i)}}{\partial \phi} K_{16}^{(i)}+\frac{\partial p_{y}^{(i)}}{\partial \phi} K_{26}^{(i)}+\frac{\partial p_{z}^{(i)}}{\partial \phi} K_{36}^{(i)} \\
& -\frac{\partial^{2} p_{x}^{(i)}}{\partial \phi \partial \psi} F_{H}^{(i)} \cos \alpha^{(i)}-\frac{\partial^{2} p_{y}^{(i)}}{\partial \phi \partial \psi} F_{H}^{(i)} \sin \alpha^{(i)}+\frac{\partial^{2} p_{z}^{(i)}}{\partial \phi \partial \psi} F_{V}^{(i)}
\end{aligned}
$$

- Fifth line coefficients - coefficients associated with a displacement $\theta$ :

$$
\begin{aligned}
K_{51}^{(i)}= & \frac{\partial p_{x}^{(i)}}{\partial \theta} K_{11}^{(i)}+\frac{\partial p_{y}^{(i)}}{\partial \theta} K_{12}^{(i)}+\frac{\partial p_{z}^{(i)}}{\partial \theta} K_{13}^{(i)} \\
K_{52}^{(i)}= & \frac{\partial p_{x}^{(i)}}{\partial \theta} K_{21}^{(i)}+\frac{\partial p_{y}^{(i)}}{\partial \theta} K_{22}^{(i)}+\frac{\partial p_{z}^{(i)}}{\partial \theta} K_{23}^{(i)} \\
K_{53}^{(i)}= & \frac{\partial p_{x}^{(i)}}{\partial \theta} K_{31}^{(i)}+\frac{\partial p_{y}^{(i)}}{\partial \theta} K_{32}^{(i)}+\frac{\partial p_{z}^{(i)}}{\partial \theta} K_{33}^{(i)} \\
K_{54}^{(i)}= & \frac{\partial p_{x}^{(i)}}{\partial \theta} K_{14}^{(i)}+\frac{\partial p_{y}^{(i)}}{\partial \theta} K_{24}^{(i)}+\frac{\partial p_{z}^{(i)}}{\partial \theta} K_{34}^{(i)} \\
K_{55}^{(i)}= & \frac{\partial p_{x}^{(i)}}{\partial \theta} K_{15}^{(i)}+\frac{\partial p_{y}^{(i)}}{\partial \theta} K_{25}^{(i)}+\frac{\partial p_{z}^{(i)}}{\partial \theta} K_{35}^{(i)} F_{H}^{(i)} \cos \alpha^{(i)}-\frac{\partial^{2} p_{y}^{(i)}}{\partial \theta \partial \phi} F_{H}^{(i)} \sin \alpha^{(i)}+\frac{\partial^{2} p_{z}^{(i)}}{\partial \theta \partial \phi} F_{V}^{(i)} \\
\quad-\frac{\partial^{2} p_{x}^{(i)}}{\partial \theta^{2}} F_{H}^{(i)} \cos \alpha^{(i)}-\frac{\partial^{2} p_{y}^{(i)}}{\partial \theta^{2}} F_{H}^{(i)} \sin \alpha^{(i)}+\frac{\partial^{2} p_{z}^{(i)}}{\partial \theta^{2}} F_{V}^{(i)} & \frac{\partial p_{x}^{(i)}}{\partial \theta} F_{H}^{(i)} \cos \alpha^{(i)}-\frac{\partial^{2} p_{y}^{(i)}}{\partial \theta \partial \psi} F_{H}^{(i)} \sin \alpha^{(i)}+\frac{\partial^{2} p_{z}^{(i)}}{\partial \theta \partial \psi} F_{V}^{(i)} \\
K_{56}^{(i)}= & \frac{\partial p_{y}^{(i)}}{\partial \theta} K_{26}^{(i)}+\frac{\partial p_{z}^{(i)}}{\partial \theta} K_{36}^{(i)}
\end{aligned}
$$


- Sixth line coefficients - coefficients associated with a displacement $\psi$ :

$$
\begin{aligned}
& K_{61}^{(i)}=\frac{\partial p_{x}^{(i)}}{\partial \psi} K_{11}^{(i)}+\frac{\partial p_{y}^{(i)}}{\partial \psi} K_{12}^{(i)} \\
& K_{62}^{(i)}=\frac{\partial p_{x}^{(i)}}{\partial \psi} K_{21}^{(i)}+\frac{\partial p_{y}^{(i)}}{\partial \psi} K_{22}^{(i)} \\
& K_{63}^{(i)}=\frac{\partial p_{x}^{(i)}}{\partial \psi} K_{31}^{(i)}+\frac{\partial p_{y}^{(i)}}{\partial \psi} K_{32}^{(i)} \\
& K_{64}^{(i)}=\frac{\partial p_{x}^{(i)}}{\partial \psi} K_{14}^{(i)}+\frac{\partial p_{y}^{(i)}}{\partial \psi} K_{24}^{(i)}-\frac{\partial^{2} p_{x}^{(i)}}{\partial \psi \partial \phi} F_{H}^{(i)} \cos \alpha^{(i)}-\frac{\partial^{2} p_{y}^{(i)}}{\partial \psi \partial \phi} F_{H}^{(i)} \sin \alpha^{(i)} \\
& K_{65}^{(i)}=\frac{\partial p_{x}^{(i)}}{\partial \psi} K_{15}^{(i)}+\frac{\partial p_{y}^{(i)}}{\partial \psi} K_{25}^{(i)}-\frac{\partial^{2} p_{x}^{(i)}}{\partial \psi \partial \theta} F_{H}^{(i)} \cos \alpha^{(i)}-\frac{\partial^{2} p_{y}^{(i)}}{\partial \psi \partial \theta} F_{H}^{(i)} \sin \alpha^{(i)} \\
& K_{66}^{(i)}=\frac{\partial p_{x}^{(i)}}{\partial \psi} K_{14}^{(i)}+\frac{\partial p_{y}^{(i)}}{\partial \psi} K_{24}^{(i)}-\frac{\partial^{2} p_{x}^{(i)}}{\partial \psi^{2}} F_{H}^{(i)} \cos \alpha^{(i)}-\frac{\partial^{2} p_{y}^{(i)}}{\partial \psi^{2}} F_{H}^{(i)} \sin \alpha^{(i)}
\end{aligned}
$$

Notice that some coefficients are expressed as functions of others. The algebraic manipulations that yielded this formulation are presented in Appendix E.

Summing up the contributions of all mooring lines, the stiffness matrix of the complete mooring system is:

$$
\mathbb{K}=\sum_{i=1}^{N} \mathbb{K}^{(i)}
$$

Looking the stiffness coefficients previously presented, it is interesting to notice some important aspects. Firstly, as it was expected, the stiffness matrix is symmetric, since forces are considered purely conservative. Indeed, as it can be seen from Eqs. 3.30 to 3.65 , coefficients $K_{j k}=K_{k j}$ as $\partial F_{H}^{(i)} / \partial v_{f}^{(i)}=\partial F_{V}^{(i)} / \partial h_{f}^{(i)}$. In addition, it is crucial to understand that the given stiffness matrix is not defined with respect to the moving frame $E_{\xi}$, fixed to the body. If the reader intents to work on this reference frame, one should rotate the result. Equation 3.67 presents this operation.

$$
\mathbb{K}_{E_{\xi}}=\mathbb{R}^{* t} \mathbb{K}_{E_{x}} \mathbb{R}^{*}
$$

where:

$$
\mathbb{R}^{*}=\left(\begin{array}{c|c}
\mathbb{R}_{E_{x} \mid E_{\xi}} & 0 \\
\hline 0 & \mathbb{I}
\end{array}\right)
$$

with $\mathbb{I}$ being the $3 \times 3$ identity matrix.

Considering that some coefficients as combinations of others, it is possible to rewrite the stiffness matrix in a more elegant way. Let us firstly define the partitions of the matrix, defining the partition representing only the translational degrees of freedom as $\mathbb{K}_{T T}^{(i)}$, the partition representing the coupling between translational and rotational motions as $\mathbb{K}_{T R}^{(i)}$ 
and the one associated with the rotational DoFs only as $\mathbb{K}_{R R}^{(i)}$. It is obvious that once the stiffness matrix is symmetric, $\mathbb{K}_{R T}^{(i)}=\mathbb{K}_{T R}^{(i)}{ }^{t}$, as follows:

$$
\mathbb{K}^{(i)}=\left(\begin{array}{c|l}
\mathbb{K}_{T T}^{(i)} & \mathbb{K}_{T R}^{(i)} \\
\hline \mathbb{K}_{R T}^{(i)} & \mathbb{K}_{R R}^{(i)}
\end{array}\right)
$$

Considering coefficients from partition $\mathbb{K}_{T R}^{(i)}$, it is possible - as showed at the respective equations - to rewrite them as:

$$
\mathbb{K}_{T R}^{(i)}=\mathbb{K}_{T T}^{(i)} \cdot\{\nabla\}\left(\mathbb{R}_{E_{x} \mid E_{\xi}} \cdot\left\{\overrightarrow{P^{(i)}}\right\}_{E_{\xi}}\right)
$$

with:

$$
\boldsymbol{\nabla}[\quad](\phi, \theta, \psi)=\left(\frac{\partial[]}{\partial \phi}(\phi, \theta, \psi), \frac{\partial[]}{\partial \theta}(\phi, \theta, \psi), \frac{\partial[\quad]}{\partial \psi}(\phi, \theta, \psi)\right)
$$

Now, let us focus on the partition $\left[\mathbb{K}_{R R}\right]$. From the aforementioned coefficients, one can write:

$$
\mathbb{K}_{R R}^{(i)}=\mathbb{K}_{R R}^{(i)}{ }^{*}-\overline{\mathbb{K}}_{R R}^{(i)}
$$

with:

$$
\begin{aligned}
\mathbb{K}_{R R}^{(i)}{ }^{*}=\nabla^{t}\left(\mathbb{R}_{E_{x} \mid E_{\xi}} \cdot\left\{\overrightarrow{P^{(i)}}\right\}_{E_{\xi}}\right) \cdot \mathbb{K}_{T R}^{(i)}= \\
\nabla^{t}\left(\mathbb{R}_{E_{x} \mid E_{\xi}} \cdot\left\{\overrightarrow{P^{(i)}}\right\}_{E_{\xi}}\right) \cdot\left[\mathbb{K}_{T T}^{(i)} \cdot \nabla\left(\mathbb{R}_{E_{x} \mid E_{\xi}} \cdot\left\{\overrightarrow{P^{(i)}}\right\}_{E_{\xi}}\right)\right]
\end{aligned}
$$

and

$$
\overline{\mathbb{K}}_{R R}^{(i)}=\frac{\partial^{2} p_{x}^{(i)}}{\partial \boldsymbol{\theta}^{2}} F_{H}^{(i)} \cos \alpha^{(i)}+\frac{\partial^{2} p_{y}^{(i)}}{\partial \boldsymbol{\theta}^{2}} F_{H}^{(i)} \sin \alpha^{(i)}-\frac{\partial^{2} p_{z}^{(i)}}{\partial \boldsymbol{\theta}^{2}} F_{V}^{(i)}
$$

remembering that $\boldsymbol{\theta}=\left[\begin{array}{lll}\phi & \theta & \psi\end{array}\right]^{t}$.

Thus, one may conclude that the stiffness coefficients associated with the translationrotation and rotation only motions are functions of the stiffness coefficients associated with the translational DoFs.

As a particular but important case, let us consider a perfect polar symmetric mooring system. A polar symmetry, in the unloaded condition, is herein defined by taking a vertical axis passing through the pole $G$ with all fairleads equidistant to it. All mooring lines are identical and their planes intercept the symmetry axis. In other words, it is a regular polygonal arrangement. This is the case of the OC4 platform, studied in Chapter 4; see Fig. 36. In this scenario, it is possible to show that the only non-null mooring stiffness coefficients for a $N$-line symmetric arrangement becomes:

$$
K_{11}=\frac{N}{2}\left(k_{H H}+\bar{k}_{H H}\right)
$$




$$
\begin{aligned}
& K_{15}=K_{51}=\frac{N}{2}\left(k_{V H} R_{f}+k_{H H} p_{\zeta}+\bar{k}_{H H} p_{\zeta}\right) \\
& K_{22}=\frac{N}{2}\left(k_{H H}+\bar{k}_{H H}\right) \\
& K_{24}=K_{42}=-\frac{N}{2}\left(k_{V H} R_{f}+k_{H H} p_{\zeta}+\bar{k}_{H H} p_{\zeta}\right) \\
& K_{33}=N k_{V V} \\
& K_{44}=\frac{N}{2}\left(p_{\zeta}^{2} k_{H H}+p_{\zeta}^{2} \bar{k}_{H H}+2 p_{\zeta} R_{f} k_{H V}+R_{f}^{2} k_{V V}+R_{f} F_{H}-2 p_{\zeta} F_{V}\right) \\
& K_{55}=\frac{N}{2}\left(p_{\zeta}^{2} k_{H H}+p_{\zeta}^{2} \bar{k}_{H H}+2 p_{\zeta} R_{f} k_{H V}+R_{f}^{2} k_{V V}+R_{f} F_{H}-2 p_{\zeta} F_{V}\right) \\
& K_{66}=N \bar{k}_{H H} R_{f}^{2}\left(1+\frac{h}{R_{f}}\right)
\end{aligned}
$$

where $R_{f}$ radius from the platform center to fairleads.

Notice that the "string stiffness" is indeed significant for this scenario. Pesce, Amaral and Franzini (2018) point out that when the pre-tensioning of the mooring lines is high, as for example in a taut-leg mooring system, it becomes even more relevant.

Finally, one last remark must be made. The presented methodology can be compared with the stiffness matrix proposed by Pesce, Amaral and Franzini (2018). Adopting the same hypothesis that the motion is restricted to the horizontal plane, the stiffness matrix herein proposed is equal to the other. Hence, the present work is an extension of the previous findings of Pesce, Amaral and Franzini (2018). This comparison is presented in Appendix F.

\subsection{Multi-segment mooring line model}

This current section proposes a model for the tension-displacement relation for a generic multi-segment (MS) mooring line. Thus, the so-called characteristic tension curves (from Equation 3.13) are introduced for this model. Once more, it is important to remark that the stiffness matrix formulation presented in Section 2.4 is general, i.e. it does not depend on the model presented at this section. Indeed, the mooring line can have any possible profile, only being necessary to know its characteristic tension function. However, looking forward for immediate applications at the case studies, it is important to have a close formulation.

Let us define a MS mooring line as a combination of multiple extensible catenary mooring segments connected. Figure 30 brings a sketch of the mooring line profile. Let us define a local coordinate system $A_{h v}$, with $h$ and $v$ being the horizontal and vertical axes, oriented as in Figure 30. The horizontal and vertical distances between anchor $A$ and fairlead $P$ are $h_{f}$ and $v_{f}$, respectively. In their turns, the projections of each segment onto both axes are $h_{j}$ and $v_{j}$. Each segment is composed by a linear elastic material with 
unstretched suspended length $l_{s_{j}}{ }^{4}$, axial stiffness $E A_{j}$ and equivalent immersed weight per unit length $\gamma_{j}$. For the present work, it is considered the case in which a portion of the first segment might rest at the seabed, i.e. there is a touchdown point (TDP). Then, the total unstretched length of the first segment is:

$$
l_{1}=l_{r_{1}}+l_{s_{1}}
$$

where $l_{r_{1}}$ is the unstretched length of the portion of the first segment that rests at the seabed.

Figure 30 - Multi-segment mooring line profile.

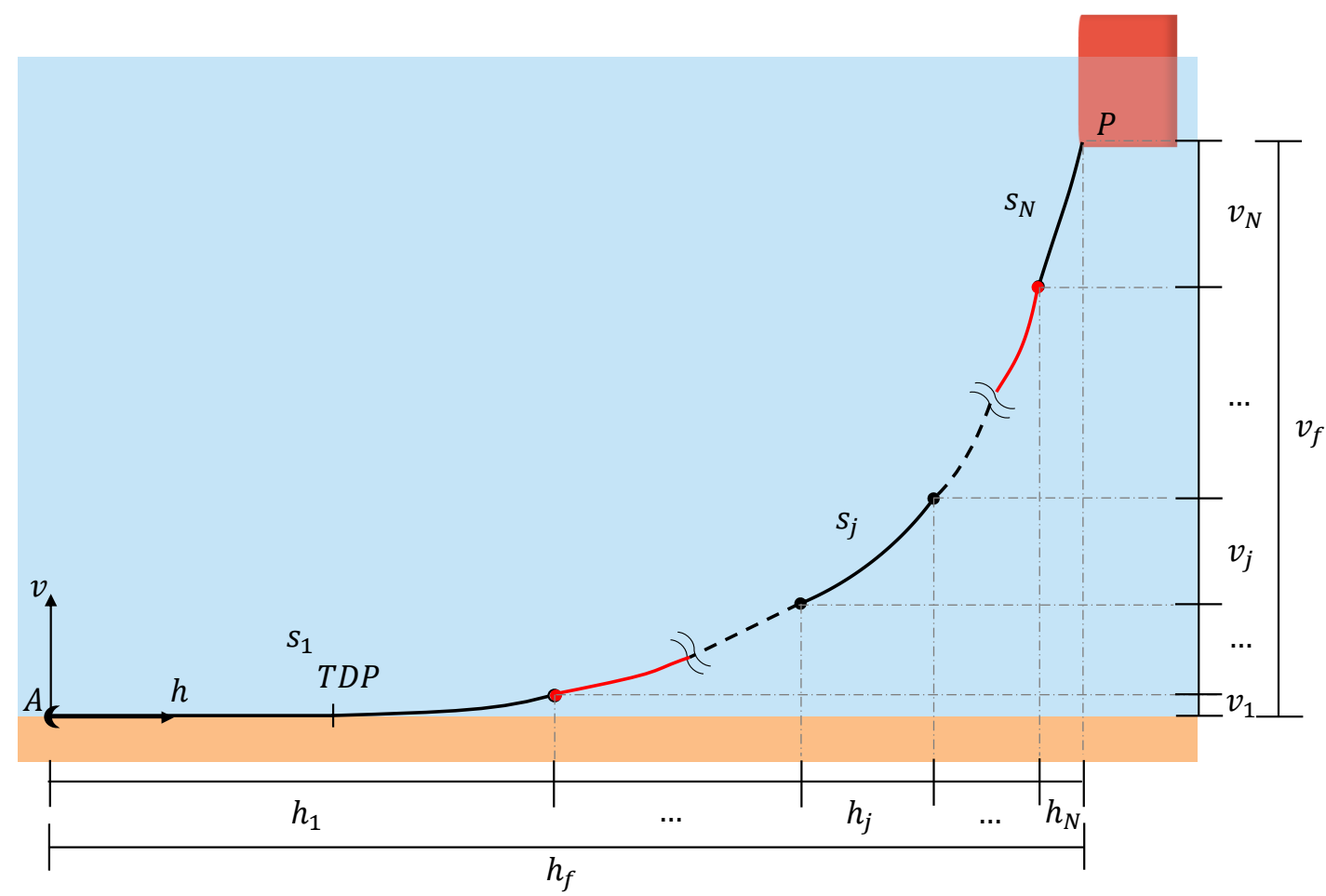

Source: The Author

Let us now consider an infinitesimal element of the segment $s_{j}$, as presented at Figure 31. Considering the linear elasticity of the material, it is possible to relate the stretched and the unstretched lengths of the element ( $d \tilde{s}$ and $d s$, respectively):

$$
\frac{d \tilde{s}_{j}}{d s_{j}}=1+\frac{T}{E A_{j}}
$$

From the horizontal equilibrium of the forces and after some algebraic manipulations, it is derived:

$$
\frac{d T}{T}=\tan \theta d \theta
$$

4 In this context, unstretched length can be seen as the nominal unloaded length. 
Figure 31 - Forces acting on an infinitesimal mooring element.

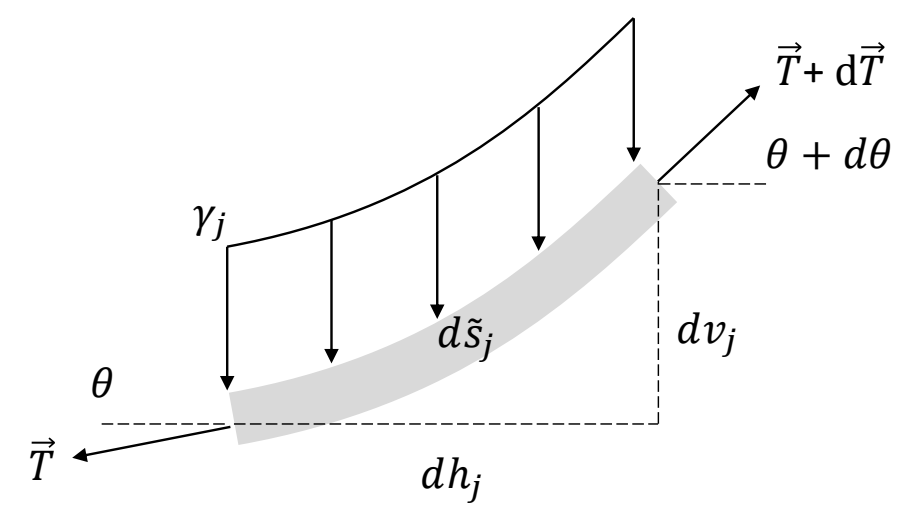

Source: The Author

Then, one may find the horizontal component of the tension is constant:

$F_{H}=T \cos \theta$

On the other hand, the vertical equilibrium of the forces leads to:

$\gamma_{j} d s_{j}=d T \sin \theta+T \cos \theta d \theta$

Considering Equation 3.85 and with a simple algebraic work, Equation 3.87 becomes:

$\gamma_{j} d s_{j}=\frac{F_{H}}{\cos ^{2} \theta} d \theta$

From Figure 31, the geometric relations are written:

$$
\begin{aligned}
& \frac{d h_{j}}{d \tilde{s}_{j}}=\cos \theta \\
& \frac{d v_{j}}{d \tilde{s}_{j}}=\sin \theta
\end{aligned}
$$

A classic result from extensible catenary laying cables can be obtained by combining Equation 3.84 and Equations 3.89 and 3.90, respectively:

$$
\begin{aligned}
& \frac{d h_{j}}{d s_{j}}=\cos \theta+\frac{F_{H}}{E A_{j}} \\
& \frac{d v_{j}}{d s_{j}}=\sin \theta+\frac{T \sin \theta}{E A_{j}}
\end{aligned}
$$

where: $F_{H}$ is the horizontal projection of the tension at the fairlead. Also, from classical catenary formulations, $F_{H}$ can be shown to be constant along the cable (Faltinsen, 1993). 
Now, the distances $h_{j}$ and $v_{j}$ are calculated by integrating, respectively, Equations 3.91 and 3.92 considering Equation 3.88:

$$
\begin{aligned}
& h_{j}=\frac{F_{H}}{\gamma_{j}}\left[\ln \left(\frac{F_{V_{m}}+\sqrt{F_{H}^{2}+F_{V_{m}}^{2}}}{F_{H}}\right)-\ln \left(\frac{F_{V_{m-1}}+\sqrt{F_{H}^{2}+F_{V_{m-1}}^{2}}}{F_{H}}\right)\right]+\frac{F_{H}}{E A_{j}} l_{s_{j}} \\
& v_{j}=\frac{1}{\gamma_{j}}\left(\sqrt{F_{H}^{2}+F_{V_{m}}^{2}}-\sqrt{F_{H}^{2}+F_{V_{m-1}}^{2}}\right)+\frac{F_{V_{m}}}{E A_{j}} l_{s_{j}}-\frac{1}{2} \frac{l_{s_{j}}^{2} \gamma_{j}}{E A_{j}}
\end{aligned}
$$

where: $F_{V_{m-1}}$ and $F_{V_{m}}$ are the vertical component of the tension at the lower and upper extremities of the segment, respectively, as presented at Figure 32.

Figure 32 - Free-body diagram for $s_{1}$ and $s_{j}$.

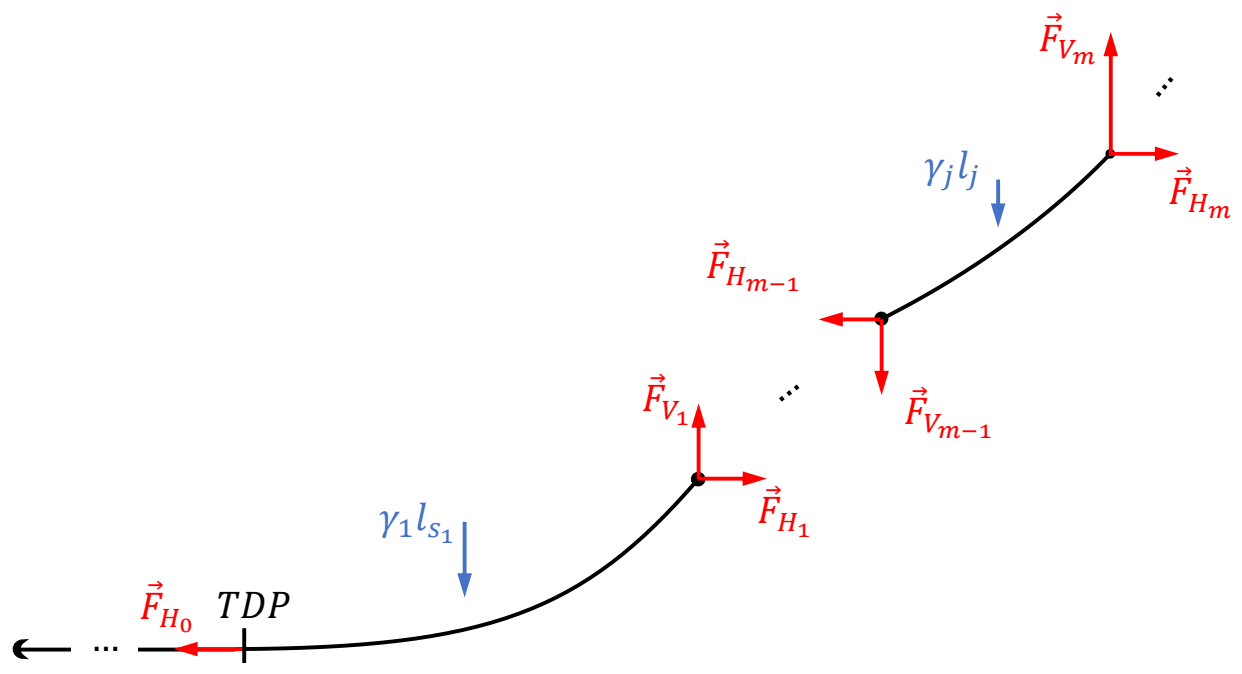

Source: The Author

Figure 32 illustrates the free-body diagram of segments $s_{1}$ and $s_{j}$. Notice that the equilibrium at the horizontal direction brings $F_{H_{m}}=F_{H_{m-1}}=\ldots=F_{H}$, i.e. the horizontal component of the tension is constant along the segment, as demonstrated in Equation 3.86. Here, as aforementioned, two hypotheses are taken into consideration: the friction between the line and the seabed is neglected, and there is no sea current loading. The portion resting is then submitted to a constant axial tension $F_{H}$. Nevertheless, from the vertical equilibrium, it is trivial to notice that ${ }^{5}$ :

$$
F_{V_{m}}=F_{V_{m-1}}+\gamma_{j} l_{s_{j}}
$$

By the action-reaction principle and taking into consideration the above force relations, it is clear to see that the vertical force acting at the vessel fairlead can be written

$\overline{5}$ Notice that for the case where there is an TDP, i.e. a portion of the first segment lays at the seabed, $F_{V_{0}}=0$. 
as function of the vertical component of the forces at the upper ends of each segment. At the same time, as the horizontal component is constant along the line, it follows that:

$$
\begin{aligned}
& F_{H}=F_{H_{N}} \\
& F_{V}=F_{V_{N}}=F_{V_{N-1}}+l_{s_{N}} \gamma_{N}
\end{aligned}
$$

Then, the vertical force $F_{V_{m}}$ can be expressed as:

$$
F_{V_{m}}=F_{V}-\sum_{k=j+1}^{N} l_{s_{k}} \gamma_{k}
$$

From Equations 3.95 and 3.98, it is possible to obtain the unstretched suspended length of the first segment:

$$
l_{s 1}=\frac{1}{\gamma_{1}}\left(F_{V}-\sum_{k=2}^{N} l_{s_{k}} \gamma_{k}\right)
$$

Now, it is possible to rewrite Equations 3.93 and 3.94, as functions of the forces at the fairlead:

$$
\begin{aligned}
& h_{j}=\frac{F_{H}}{\gamma_{j}} {\left[\ln \left(\frac{F_{V}-\sum_{k=j+1}^{N} l_{s_{k}} \gamma_{k}+\sqrt{F_{H}^{2}+\left(F_{V}-\sum_{k=j+1}^{N} l_{s_{k}} \gamma_{k}\right)^{2}}}{F_{H}}\right)\right.} \\
&\left.-\ln \left(\frac{F_{V}-\sum_{k=j}^{N} l_{s_{k}} \gamma_{k}+\sqrt{F_{H}^{2}+\left(F_{V}-\sum_{k=j}^{N} l_{s_{k}} \gamma_{k}\right)^{2}}}{F_{H}}\right)\right]+\frac{F_{H}}{E A_{j}} l_{s_{j}} \\
& v_{j}=\frac{1}{\gamma_{j}}\left(\sqrt{F_{H}^{2}+\left(F_{V}-\sum_{k=j+1}^{N} l_{s_{k}} \gamma_{k}\right)^{2}}-\sqrt{F_{H}^{2}+\left(F_{V}-\sum_{k=j}^{N} l_{s_{k}} \gamma_{k}\right)^{2}}\right) \\
&+\frac{F_{V}-\sum_{k=j+1}^{N} l_{s_{k}} \gamma_{k}}{E A_{j}} l_{s_{j}}-\frac{1}{2} \frac{l_{s_{j}}^{2} \gamma_{j}}{E A_{j}}
\end{aligned}
$$

For the case where the line touches the seabed, the vertical projection of the first segment is given considering the relation from Equation 3.99:

$$
v_{1}=\frac{1}{\gamma_{1}} \sqrt{F_{H}^{2}+\left(F_{V}-\sum_{k=2}^{N} l_{s_{k}} \gamma_{k}\right)^{2}}+\frac{1}{2} \frac{\left(F_{V}-\sum_{k=2}^{N} l_{s_{k}} \gamma_{k}\right)^{2}}{E A_{1} \gamma_{1}}
$$

Again, for the case with TDP, the remaining term to be defined is the horizontal projection of the portion resting at the seabed of the first segment. Firstly, the unstretched length can be obtained by combining Equations 3.83 and 3.98.

$$
l_{r 1}=l_{1}-\frac{1}{\gamma_{1}}\left(F_{V}-\sum_{k=2}^{N} l_{s_{k}} \gamma_{k}\right)
$$


Then, from the linear elasticity, the projection of the portion resting at the seabed ${ }^{6}$ is:

$$
h_{1}^{r}=\left[l_{1}-\frac{1}{\gamma_{1}}\left(F_{V}-\sum_{k=2}^{N} l_{s_{k}} \gamma_{k}\right)\right]\left(1+\frac{F_{H}}{E A_{1}}\right)
$$

Finally, the horizontal projection of the first segment as a whole is:

$$
\begin{aligned}
h_{1}=l_{1}-\frac{1}{\gamma_{1}}\left(F_{V}\right. & \left.-\sum_{k=2}^{N} l_{s_{k}} \gamma_{k}\right)+\frac{F_{H}}{E A_{1}} l_{1} \\
& \quad+\frac{F_{H}}{\gamma_{1}} \ln \left(\frac{F_{V}-\sum_{k=2}^{N} l_{s_{k}} \gamma_{k}+\sqrt{F_{H}^{2}+\left(F_{V}-\sum_{k=2}^{N} l_{s_{k}} \gamma_{k}\right)^{2}}}{F_{H}}\right)
\end{aligned}
$$

Concluding, one may write the expressions of the horizontal and vertical projections of the anchor-fairlead distance as functions of the horizontal and vertical components of the tension at the fairlead, as in Equations 3.106 and 3.107. These equations are the so-called characteristic tension function for each multi-segment mooring line.

$$
\begin{aligned}
& h_{f}\left(F_{H}, F_{V}\right)=\sum_{j=1}^{N} h_{j} \\
& v_{f}\left(F_{H}, F_{V}\right)=\sum_{j=1}^{N} v_{j}
\end{aligned}
$$

Some important considerations must be made. In case of modelling a one-segment extensible catenary mooring line, i.e. considering $N=1$, one may reduce the model above to only the first segment. The resulting formulation is exactly the classic one founded, for example, in Jonkman (2007). In turn, the classical (non-extensible) catenary line model can be obtained by considering the $E A_{1} \rightarrow \infty$. The terms related to the linear elasticity will be reduced to zero, and final distances become functions of the tension only. It is easy to notice that this scenario makes the line "stiffer", and this effect is deeper discussed at Chapter 4 .

6 Notice that the horizontal projection of this portion is numerically equal to its stretched length. 



\section{Analyses of Spread Mooring Systems}

The present chapter aims to illustrate and discuss the application of the proposed methodologies in this master dissertation. In this context, the OC4-DeepCwind semisubmersible platform is taken as a case study. Two SMS arrangements are considered: (i) an one-segment all-chain catenary mooring system with three lines and (ii) a three-segment chain-wire-chain catenary mooring system with six lines. The first case study follows the original arrangement proposed in Robertson et al. (2014). For this case study, the influence of the mooring line pre-tensioning is investigated by considering different pre-tensioning scenarios. The effect of the line axial stiffness is also studied. On the second case study, the concept proposed in the second case study allows to understand the use of the multisegment mooring line model, illustrating the application of the formulation as a design tool.

Different analyses are herein derived for both cases. Firstly, the stiffness matrix for the trivial equilibrium position ${ }^{1}$ is calculated. Secondly, the natural periods and the equivalent modes of oscillation for the horizontal plane motions $\left(\left[\begin{array}{lll}r_{x} & r_{y} & \psi\end{array}\right]\right)$ are evaluated by simply solving the corresponding linear eigenvalue problem. Thirdly, the effect of the vessel offset and heading on the results is discussed by plotting colored maps, justifying the importance of a formulation for a generic position different from the trivial equilibrium one. Now, the OC4 project is briefly described, with focus on the relevant parts for this work. More details are available in Robertson et al. (2014a).

Figure 33 presents the OC4 floating unit and tower. The semisubmersible-type floating unit is composed of three large columns disposed on the form of an equilateral triangle and a central column in which the tower is attached. This configuration yields a centralized center of mass. The connection between the columns is made by means of a set of pontoons and cross members. The base of the columns are enlarged. Table 3 brings both the floating unit structural and hydrodynamic properties. Notice that only the planar motions properties are presented, since only they are investigated in the linear eigenvalue problem. The asymptotic limit of zero-frequency was adopted for a first estimation of the added masses and moments of inertia for those motions. This hypothesis is justified as the periods of oscillation for the planar modes are typically higher then $100 \mathrm{~s}$, and considering that for this range of periods the added masses tend to be constant, as it can be seen in Figure 34. The values have been determined through the worldwide-recognized panel method-based software WAMITR. These values were obtained from Robertson et al. (2014a).

1 Here, the trivial equilibrium position means the position when no external force acts at the system, i.e. the design configuration. 
Figure 33 - The OC4-DeepCwind floating wind system design.

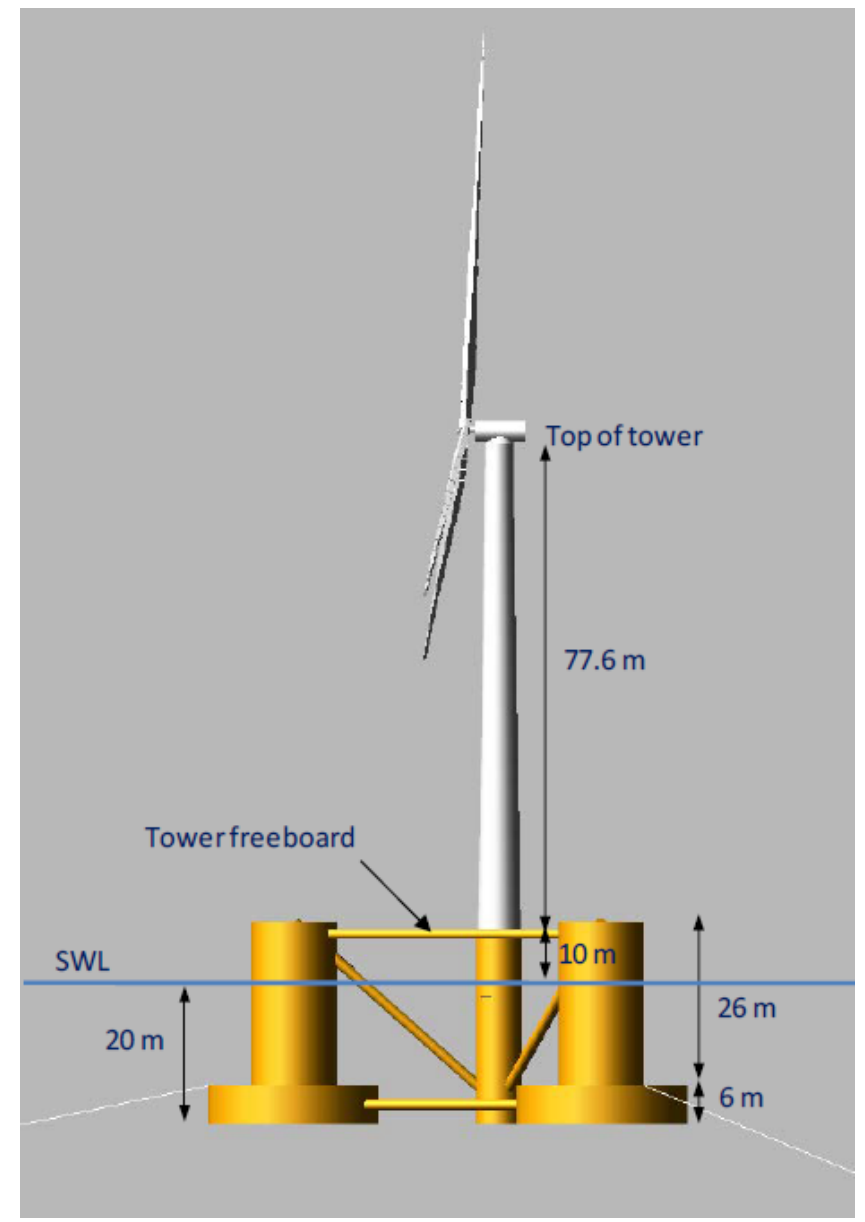

Source: Robertson et al. (2014a)

Before going to the case studies themselves, Figure 35 brings the step-by-step procedure for evaluating the stiffness matrix using the formulation herein proposed. In the figure, keypoints denote the position of the mooring lines anchors and fairleads, with respect to the global and local references frames, respectively. The second step can be

Table 3 - Platform structural and hydrodynamic properties.

\begin{tabular}{ll}
\hline \hline Type & Semisub. \\
Mass $(m)$ & $1.3473 \mathrm{E}+7 \mathrm{~kg}$ \\
Platform yaw inertia about CM $\left(I_{\psi \psi}\right)$ & $1.226 \mathrm{E}+10 \mathrm{kgm}^{2}$ \\
Surge-Surge added mass $\left(M_{a \xi \xi}\right)$ & $8.47 \mathrm{E}+6 \mathrm{~kg}$ \\
Sway-Sway added mass $\left(M_{a \eta \eta}\right)$ & $8.47 \mathrm{E}+6 \mathrm{~kg}$ \\
Yaw-Yaw added mass $\left(M_{a \psi \psi}\right)$ & $6.44 \mathrm{E}+9 \mathrm{kgm}^{2}$ \\
\hline \hline
\end{tabular}

Source: Adapted from (Robertson et al., 2014a) 
Figure 34 - The OC4-DeepCwind floating wind added masses and moment of inertia for the planar motions. Determined with WAMITR.

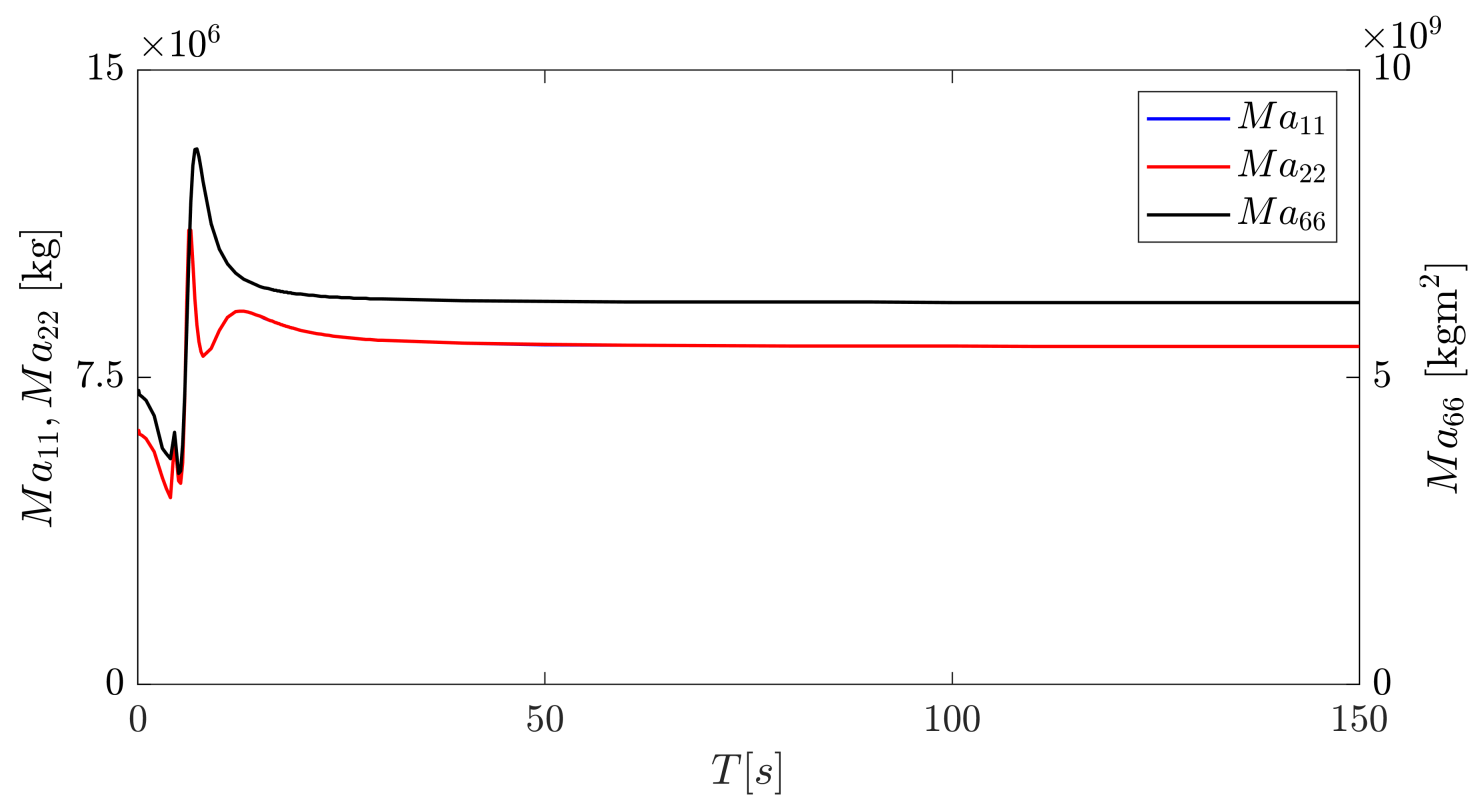

Source: The Author

1. Mooring system definition: keypoints, number of lines, line composition

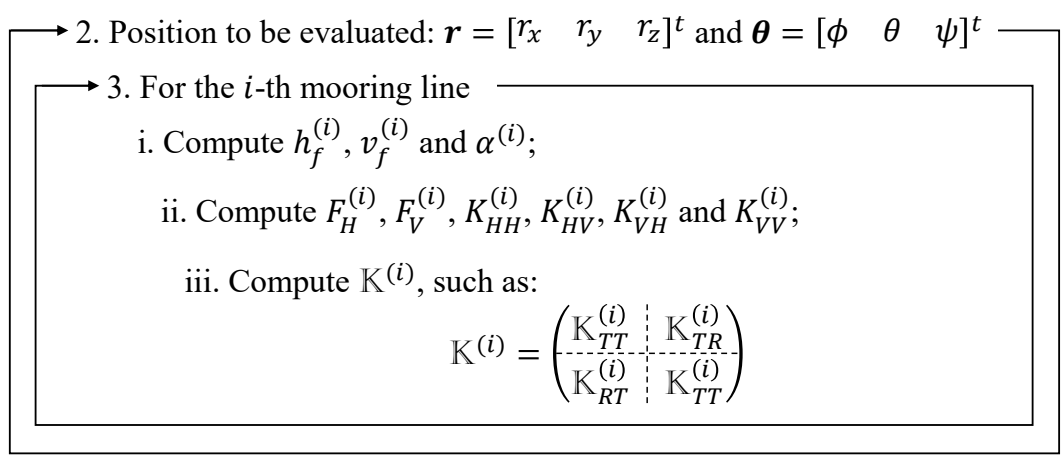

Figure 35 - Step-by-step procedure for the mooring system stiffness calculation.

used in a recursive way, allowing users to determine the colormaps presented in Sections 4.1 and 4.2, or just evaluating the stiffness matrix at an specific position (being the trivial equilibrium position or not). Finally, it is important to highlight once more that to compute the $i$-th mooring line contribution for the whole system stiffness matrix, its characteristic tension functions $\left(F_{H}^{(i)}\left(h_{f}^{(i)}, v_{f}^{(i)}\right)\right.$ and $\left.F_{V}^{(i)}\left(h_{f}^{(i)}, v_{f}^{(i)}\right)\right)$ have to be known a priori, seen as inputs of the problem. For some cases, such as catenary-laying cables, the analytical formulation is known. When not, the characteristic tension functions might be determined using numerical methods, e.g. FE modeling.

Once the vessel parameters are defined and the procedure is recapitulated, the proposed methodology is applied considering two different mooring line configurations. 
Section 4.1 uses the original all-chain catenary mooring line defined by Robertson et al. (2014a). In turn, in Section 4.2 an alternative three-segment hybrid mooring line is proposed, considering an hypothetical case of deep water installation. In both cases, a simple linear modal analysis is done, in order to investigate the effects of the mooring system parameters on the natural modes and periods of the system.

\subsection{All-chain catenary mooring line}

The original OC4-DeepCwind mooring arrangement proposed by Robertson et al. (2014a) is a equidistant $3 \times 1$ spread mooring system. Figure 36 brings its top view. In turn, the mooring line profile is an one-segment mooring chain, represented in Figure 37. Table 4 brings the mooring system's relevant data.

Figure 36 - Top view of the OC4-DeepCwind floating wind original mooring system.

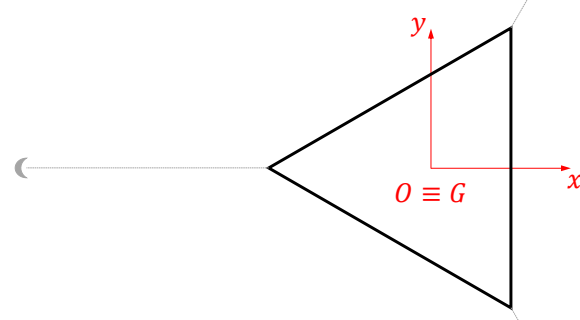

Source: Robertson et al. (2014a)

The trivial equilibrium position, i.e. the one for a system under no external excitation, is obviously $\mathbf{q}=\left[\begin{array}{llllll}0 & 0 & 0 & 0 & 0 & 0\end{array}\right]^{t}$. Notice that the system is perfectly polar symmetric. Hence, the stiffness coefficients can be written from Equations 3.75 to 3.82. It follows that the only non-null coefficients are:

$$
\begin{aligned}
K_{11} & =\frac{3}{2}\left(k_{H H}+\bar{k}_{H H}\right) \\
K_{15} & =K_{51}=\frac{3}{2}\left(k_{V H} R_{f}+k_{H H} p_{\zeta}+\bar{k}_{H H} p_{\zeta}\right) \\
K_{22} & =\frac{3}{2}\left(k_{H H}+\bar{k}_{H H}\right)
\end{aligned}
$$


Figure 37 - One-segment all-chain mooring line profile of the OC4-DeepCwind floating wind original mooring system.

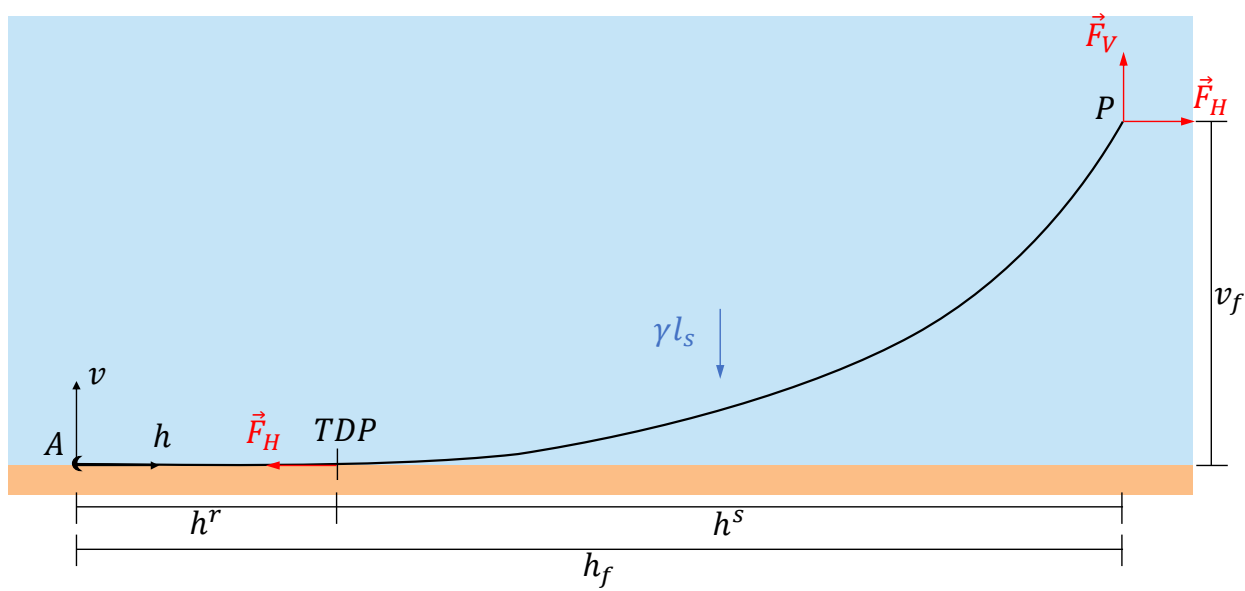

Source: The Author

Table 4 - The OC4-DeepCwind original mooring system parameters.

\begin{tabular}{lr}
\hline \hline Number of mooring lines & 3 \\
System type & Spread system \\
Line profile & One-segment \\
Line composition & Chain \\
Water depth & $200 \mathrm{~m}$ \\
Fairlead depth & $14 \mathrm{~m}$ \\
Radius from center to anchors & $837.6 \mathrm{~m}$ \\
Radius from center to fairleads & $40.9 \mathrm{~m}$ \\
Unstretched lenght & $835.35 \mathrm{~m}$ \\
Mass per unit length & $113.35 \mathrm{~kg} / \mathrm{m}$ \\
Equivalent diameter & $76.6 \mathrm{~mm}$ \\
Axial Stiffness & $753.6 \mathrm{MN}$ \\
\hline \hline
\end{tabular}

Source: Adapted from (Robertson et al., 2014a)

$$
\begin{aligned}
K_{24} & =K_{42}=-\frac{3}{2}\left(k_{V H} R_{f}+k_{H H} p_{\zeta}+\bar{k}_{H H} p_{\zeta}\right) \\
K_{33} & =3 k_{V V} \\
K_{44} & =\frac{3}{2}\left(p_{\zeta}^{2} k_{H H}+p_{\zeta}^{2} \bar{k}_{H H}+2 p_{\zeta} R_{f} k_{H V}+R_{f}^{2} k_{V V}+R_{f} F_{H}-2 p_{\zeta} F_{V}\right) \\
K_{55} & =\frac{3}{2}\left(p_{\zeta}^{2} k_{H H}+p_{\zeta}^{2} \bar{k}_{H H}+2 p_{\zeta} R_{f} k_{H V}+R_{f}^{2} k_{V V}+R_{f} F_{H}-2 p_{\zeta} F_{V}\right) \\
K_{66} & =3 \bar{k}_{H H} R_{f}^{2}\left(1+\frac{h}{R_{f}}\right)
\end{aligned}
$$

Aiming at numerically evaluating the stiffness matrix, it is necessary to solve the catenary problem. As aforementioned, for a one-segment mooring line the characteristic 
tension functions from Equations 3.106 and 3.107 have $N=1$, thereafter, becomes known the extensible catenary equations:

$$
\begin{aligned}
& h_{f}=l-\frac{1}{\gamma} F_{V}+\frac{F_{H}}{E A} l+\frac{F_{H}}{\gamma} \ln \left(\frac{F_{V}+\sqrt{F_{H}^{2}+F_{V}^{2}}}{F_{H}}\right) \\
& v_{f}=\frac{1}{\gamma} \sqrt{F_{H}^{2}+F_{V}^{2}}+\frac{1}{2} \frac{F_{V}^{2}}{E A \gamma}
\end{aligned}
$$

Notice that Equations 4.9 and 4.10 are written in the inverted form, i.e. the distances as function of the forces at the fairlead. For the development herein proposed, it is necessary to numerically evaluate forces as well as the tangent (in the plane of the line) and "string" (perpendicular to the line) stiffness. This can be done by using the Newton-Raphson method for instance (see Appendix A for details). Table 5 brings the values for the trivial equilibrium position.

Table 5 - The OC4-DeepCwind original mooring line forces and stiffness.

\begin{tabular}{ll}
\hline \hline Pre-tensioning & $1.16 \mathrm{E}+3 \mathrm{kN}$ \\
Horizontal force $\left(F_{H}\right)$ & $9.63 \mathrm{E}+2 \mathrm{kN}$ \\
Vertical force $\left(F_{V}\right)$ & $6.49 \mathrm{E}+2 \mathrm{kN}$ \\
Horizontal-Horizontal local stiffness $\left(k_{H H}\right)$ & $5.29 \mathrm{E}+1 \mathrm{kN} / \mathrm{m}$ \\
Vertical-Vertical local stiffness $\left(k_{V V}\right)$ & $6.84 \mathrm{kN} / \mathrm{m}$ \\
Horizontal-Vertical local stiffness $\left(k_{H V}\right)$ & $1.62 \mathrm{E}+1 \mathrm{kN} / \mathrm{m}$ \\
Horizontal-Horizontal "string stiffness" $\left(\bar{k}_{H H}\right)$ & $1.21 \mathrm{kN} / \mathrm{m}$ \\
\hline \hline
\end{tabular}

Source: The Author

Finally, the analytical mooring system stiffness $\mathbb{K}$ for the trivial equilibrium position becomes (values in $\mathrm{kN}, \mathrm{m}$ and $\mathrm{rad}$ ):

$$
\mathbb{K}=\left(\begin{array}{cccccc}
7.09 \mathrm{E}+1 & 0 & 0 & 0 & -1.07 \mathrm{E}+2 & 0 \\
0 & 7.09 \mathrm{E}+1 & 0 & 1.07 \mathrm{E}+2 & 0 & 0 \\
0 & 0 & 1.91 \mathrm{E}+1 & 0 & 0 & 0 \\
0 & 1.07 \mathrm{E}+2 & 0 & 8.73 \mathrm{E}+4 & 0 & 0 \\
-1.07 \mathrm{E}+2 & 0 & 0 & 0 & 8.73 \mathrm{E}+4 & 0 \\
0 & 0 & 0 & 0 & 0 & 1.17 \mathrm{E}+5
\end{array}\right)
$$

In the literature, Robertson et al. (2014a) calculated the stiffness matrix using the applied prescribed static offset analysis. Stiffness coefficients were calculated via centraldifferences of the force-displacement curves. These curves were obtained by imposing small perturbations around the trivial equilibrium position of the platform in its DoFs resulting in restoring forces. Then, Equation 4.12 brings the resulting stiffness matrix $\mathbb{K}_{\mathrm{NREL}}$ (values 
in $\mathrm{kN}, \mathrm{m}$ and $\mathrm{rad})$. It is easy to note that the the results obtained analytically are in excellent agreement with those found in (Robertson et al., 2014a).

$$
\mathbb{K}_{\text {NREL }}=\left(\begin{array}{cccccc}
7.08 \mathrm{E}+1 & 0 & 0 & 0 & -1.08 \mathrm{E}+2 & 0 \\
0 & 7.08 \mathrm{E}+1 & 0 & 1.08 \mathrm{E}+2 & 0 & 0 \\
0 & 0 & 1.91 \mathrm{E}+1 & 0 & 0 & 0 \\
0 & 1.07 \mathrm{E}+2 & 0 & 8.73 \mathrm{E}+4 & 0 & 0 \\
-1.07 \mathrm{E}+2 & 0 & 0 & 0 & 8.73 \mathrm{E}+4 & 0 \\
0 & 0 & 0 & 0 & 0 & 1.17 \mathrm{E}+5
\end{array}\right)
$$

The analytical formulation also allows investigating the effect of considering the axial stiffness in the stiffness matrix. To illustrate this, the classic (non-extensible) mooring model is taken into consideration, i.e. the axial stiffness $E A$ is taken at the limit $E A \rightarrow \infty$. In other words, it neglects the effects of the catenary line axial extension. Recovering the catenary equations, one will have:

$$
\begin{aligned}
& h_{f}=L-\frac{F_{V}}{\gamma}+\frac{F_{H}}{\gamma} \ln \left(\frac{F_{V}+\sqrt{F_{V}^{2}+F_{H}^{2}}}{F_{H}}\right) \\
& v_{f}=\frac{1}{\gamma}\left(\sqrt{F_{V}^{2}+F_{H}^{2}}-F_{V}\right)
\end{aligned}
$$

The stiffness matrix $\mathbb{K}_{\infty}$ for this case becomes (values in $\mathrm{kN}$, $\mathrm{m}$ and $\mathrm{rad}$ ):

$$
\mathbb{K}_{\infty}=\left(\begin{array}{cccccc}
8.12 \mathrm{E}+1 & 0 & 0 & 0 & -1.46 \mathrm{E}+2 & 0 \\
0 & 8.12 \mathrm{E}+1 & 0 & 1.46 \mathrm{E}+2 & 0 & 0 \\
0 & 0 & 2.05 \mathrm{E}+1 & 0 & 0 & 0 \\
0 & 1.46 \mathrm{E}+2 & 0 & 9.16 \mathrm{E}+4 & 0 & 0 \\
-1.46 \mathrm{E}+2 & 0 & 0 & 0 & 9.16 \mathrm{E}+4 & 0 \\
0 & 0 & 0 & 0 & 0 & 1.24 \mathrm{E}+5
\end{array}\right)
$$

As one could expect, increasing the axial stiffness of the mooring line increases also the absolute value of the stiffness coefficients of the mooring system. Indeed, the mooring line stiffness can be seen as an association of two springs in series ${ }^{2}$ : one related to the geometric stiffness (the terms from the right-hand-side of Equations 4.9 and 4.10 not related to $E A$ ) and the other to the elastic one. By taking $E A \rightarrow \infty$, the elastic stiffness tends also to infinity, what makes the line - and the whole system - stiffer. To illustrates this, Figure 38 shows the characteristic tension function for the extensible catenary model. Notice that for the trivial equilibrium position, where $\left(h_{f}, v_{f}\right)=(796.732,186) \mathrm{m}$, the mooring tension functions are highly nonlinear.

Following, Figure 39 shows the comparison between the characteristic curves for both scenarios: the extensible and the non-extensible catenary models. Here, it is

2 Notice that the total displacement of the equivalent spring can be seem as the sum of the displacement of the two equivalent springs. 
Figure 38 - The OC4-DeepCwind chain mooring line characteristic curves. Unities: N and $\mathrm{m}$.
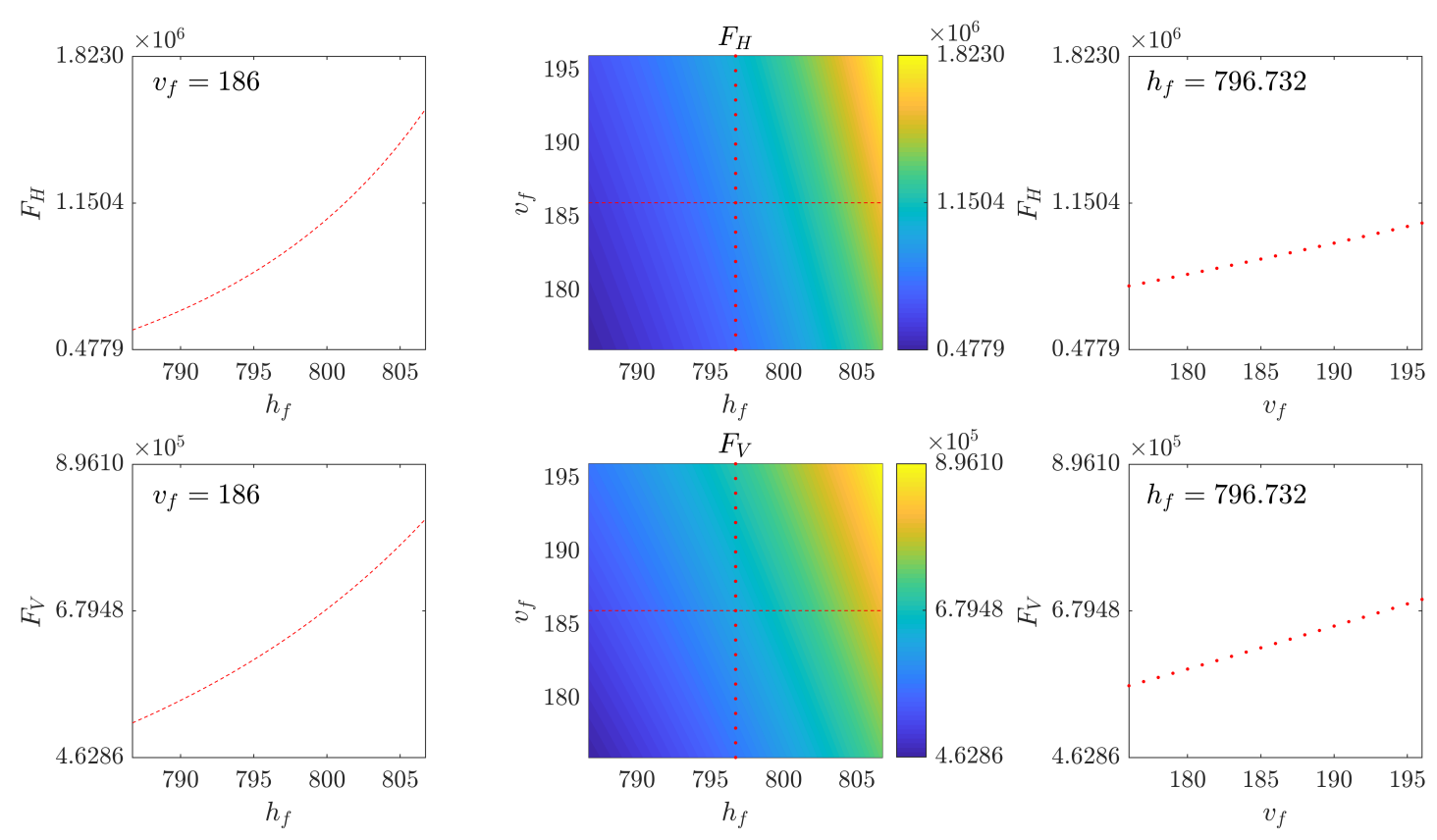

Source: The Author

remarkable the effects of the linear axial stiffness on the whole system stiffness coefficients. Notice that the derivative of the curves are higher for the curve associated with the classic non-extensible catenary. Concluding, the effect of the material extensibility is as relevant as is the geometric (catenary) stiffness. Thus, the non-consideration of the axial stiffness, following the classic (non-extensible) catenary formulation, yields an incorrect evaluation of the stiffness coefficients of the system. It is important to point out that this verification would be difficult to execute using numerical methods such as FE models. A new case study, using different properties should be modeled. Additionally, the use of models with higher stiffness (tending to infinity) might present convergence issues.

The methodology herein proposed allows also to define the vessel natural periods of oscillation, for planar motions. Table 6 brings the values for the unloaded equilibrium position and the comparison with values achieved by Robertson et al. (2014b). The natural periods are calculated by solving the classic linear eigenvalue problem. This can be done for motions on horizontal plane only, by taking the asymptotic limit at zero frequency for the values of their respective added mass coefficients. In this scenario, the corresponding $3 \times 3$ stiffness matrix recovers the one obtained in Pesce, Amaral and Franzini (2018). Notice that in the presented table, the natural periods are numerate following a crescent order and do not recovers the generalized coordinate directions. 
Figure 39 - Comparison between the characteristic curves for the extensible and the non-extensible catenary mooring models. Unities: $\mathrm{N}$ and $\mathrm{m}$.
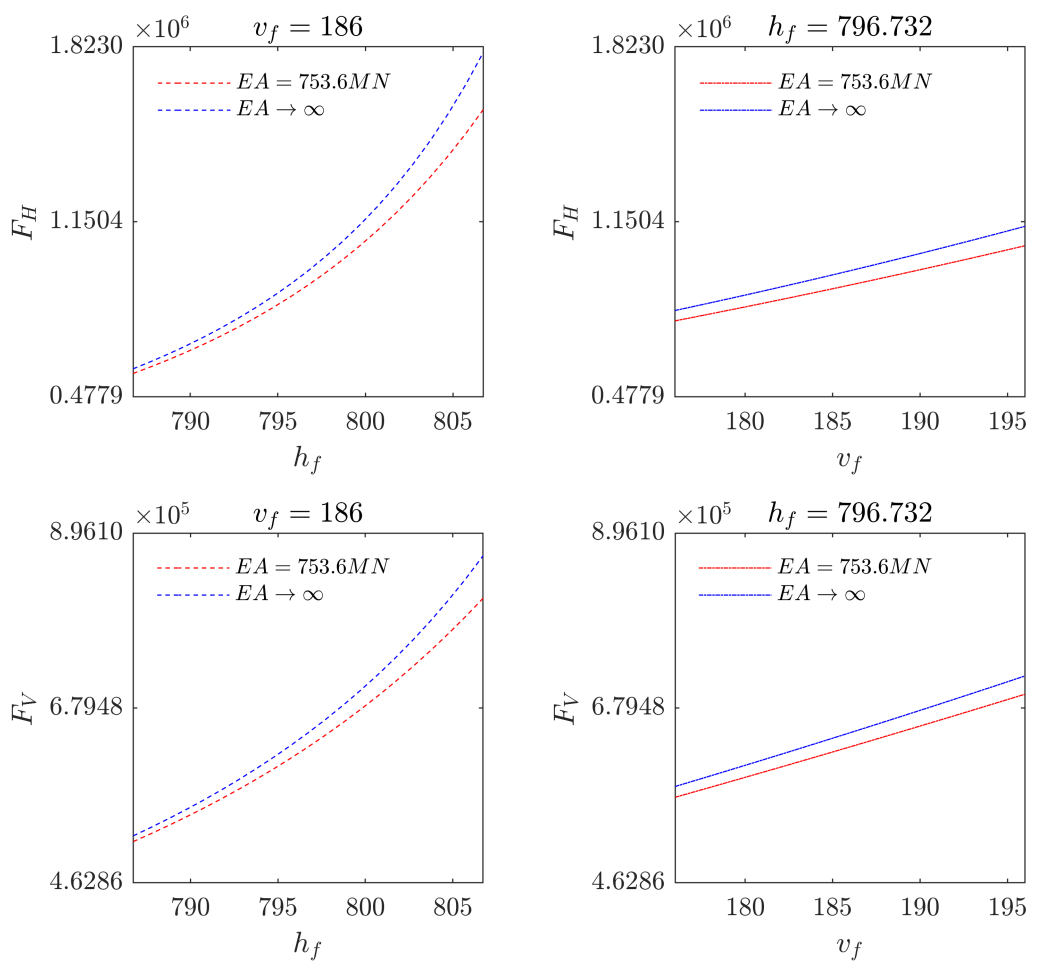

Source: The Author

Table 6 - Natural periods of oscillation for the trivial position. Unities: s.

\begin{tabular}{cccc}
\hline \hline & Extensible catenary & Robertson et al. (2014b) & Non-extensible catenary \\
\hline$T_{1}$ & 76.02 & 76.03 & 73.82 \\
$T_{2}$ & 105.48 & 105.53 & 98.57 \\
$T_{3}$ & 105.48 & 105.53 & 98.57 \\
\hline \hline
\end{tabular}

Source: The Author

As expected, the results considering the correct extensible catenary model show a good agreement with the literature. However, the classic catenary model results in lower periods, since it is stiffer than the extensible catenary model. This demonstrates again the importance of a proper line model for the analysis. In addition, note that as the periods are, indeed, high enough to consider the added masses to be constant and equal to the asymptotic limit of zero-frequency.

The modes of oscillation for the planar motions analytically obtained are sketched in Figure 40. Only the extensible catenary result is presented, since the mooring line stiffness does not affect the schematic representation of the mode shapes. Notice that 
they are "pure"-modes, i.e. there is no coupling between motions. This was expected, as the stiffness matrix considering only these motions is diagonal, for the trivial equilibrium position. In this context, it is possible to recover the ocean engineering classification, from left to right: yaw, sway and yaw.

Figure 40 - The OC4-DeepCwind modes of oscillation for the trivial position, corresponding to natural periods $T_{j}, j=1,2,3$.
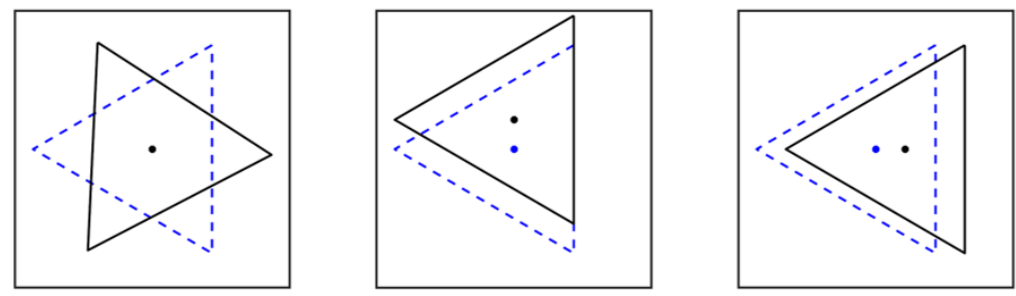

Source: The Author

Besides presenting excellent agreement with the literature results, the practical aspect of the analytical model is noteworthy. It can be used as a design tool in order to evaluate different effects of the mooring system parameters on its stiffness - the pretensioning of the mooring lines, the materials, the mooring system configuration, mooring line profile, among others. In other words, the mooring system defining parameters can be easily changed in order not only to qualify, but also quantify their effect on the whole mooring system stiffness. To illustrate this, besides the line profile changing (as presented in the next case study, in Section 4.2), let us study the effects of the mooring line pretensioning. Three scenarios are proposed: the design base case and the line being $10 \%$ less/more tensioned. Table 7 presents the important parameters for each case. In the table, $f^{*}$ is the pre-tensioning ratio with respect to the design base case. Notice that the pre-tensioning is assessed by varying the line total length.

Table 7 - Pre-tensioning cases: total length, mooring line forces and stiffness.

\begin{tabular}{llll}
\hline \hline Pre-tensioning condition & Low & Design & High \\
\hline$f^{*}$ & 0.9 & 1.0 & 1.1 \\
Length $(\mathrm{m})$ & 837.95 & 835.35 & 833.15 \\
Pre-tensioning $(\mathrm{kN})$ & $9.96 \mathrm{E}+2$ & $1.11 \mathrm{E}+3$ & $1.22 \mathrm{E}+3$ \\
Horizontal force $(\mathrm{kN})$ & $7.98 \mathrm{E}+2$ & $9.08 \mathrm{E}+2$ & $1.02 \mathrm{E}+3$ \\
Vertical force $(\mathrm{kN})$ & $5.96 \mathrm{E}+2$ & $6.31 \mathrm{E}+2$ & $6.65 \mathrm{E}+2$ \\
Horizontal-Horizontal local stiffness $(\mathrm{kN} / \mathrm{m})$ & $3.88 \mathrm{E}+1$ & $4.61 \mathrm{E}+1$ & $5.37 \mathrm{E}+1$ \\
Vertical-Vertical local stiffness $(\mathrm{kN} / \mathrm{m})$ & 6.05 & 6.38 & 6.69 \\
Horizontal-Vertical local stiffness $(\mathrm{kN} / \mathrm{m})$ & $1.29 \mathrm{E}+1$ & $1.44 \mathrm{E}+1$ & $1.60 \mathrm{E}+1$ \\
Horizontal-Horizontal "string stiffness" $(\mathrm{kN} / \mathrm{m})$ & 1.00 & 1.14 & 1.28 \\
\hline \hline
\end{tabular}


The stiffness matrix for the lower pre-tensioning condition $\left(f^{*}=0.9\right)$ case becomes (values in $\mathrm{kN}, \mathrm{m}$ and $\mathrm{rad}$ ):

$$
\mathbb{K}_{f^{*}=0.9}=\left(\begin{array}{cccccc}
5.97 \mathrm{E}+1 & 0 & 0 & 0 & -4.66 \mathrm{E}+1 & 0 \\
0 & 5.97 \mathrm{E}+1 & 0 & 4.66 \mathrm{E}+1 & 0 & 0 \\
0 & 0 & 1.81 \mathrm{E}+1 & 0 & 0 & 0 \\
0 & 4.66 \mathrm{E}+1 & 0 & 7.87 \mathrm{E}+4 & 0 & 0 \\
-4.66 \mathrm{E}+1 & 0 & 0 & 0 & 7.87 \mathrm{E}+4 & 0 \\
0 & 0 & 0 & 0 & 0 & 1.03 \mathrm{E}+5
\end{array}\right)
$$

Furthermore, the higher pre-tensioning condition $\left(f^{*}=1.1\right)$ has the following stiffness matrix (values in $\mathrm{kN}, \mathrm{m}$ and $\mathrm{rad}$ ):

$$
\mathbb{K}_{f^{*}=1.1}=\left(\begin{array}{cccccc}
8.24 \mathrm{E}+1 & 0 & 0 & 0 & -1.76 \mathrm{E}+2 & 0 \\
0 & 8.24 \mathrm{E}+1 & 0 & 1.76 \mathrm{E}+2 & 0 & 0 \\
0 & 0 & 2.01 \mathrm{E}+1 & 0 & 0 & 0 \\
0 & 1.76 \mathrm{E}+2 & 0 & 9.58 \mathrm{E}+4 & 0 & 0 \\
-1.76 \mathrm{E}+2 & 0 & 0 & 0 & 9.58 \mathrm{E}+4 & 0 \\
0 & 0 & 0 & 0 & 0 & 1.31 \mathrm{E}+5
\end{array}\right)
$$

The natural periods for the planar modes can also be calculated. Table 8 brings these results.

Table 8 - Study of the pre-tensioning effect on the natural periods of oscillation for the trivial position. Unity: s.

\begin{tabular}{cccc}
\hline \hline$f^{*}$ & 0.9 & 1.0 & 1.1 \\
\hline$T_{1}$ & 81.10 & 76.02 & 71.81 \\
$T_{2}$ & 114.97 & 105.48 & 97.78 \\
$T_{3}$ & 114.97 & 105.48 & 97.78 \\
\hline \hline
\end{tabular}

Source: The Author

By comparing the results with the design pre-tensioning condition, it is clear the influence of the line pre-tensioning on the stiffness coefficients and on the natural periods of the planar modes. Indeed, it is significant that the pre-tensioning plays an important role on the whole mooring system stiffness. Even with a small variation on these parameters, the resulting stiffness matrix presents a substantial fluctuation. For example, notice that horizontal plane motions ${ }^{3}$ present a sensitive variation on the mooring system stiffness, mainly for the yaw motion. Among other things, the loss of yaw stiffness

3 For the trivial position, the motions on the horizontal plane matches with the naval engineering "surge", "sway" and "yaw" motions, since the rotation matrix for that point is the $6 \times 6$ identity matrix. 
can cause instability of the trivial equilibrium due to external forces. This demonstrates the importance of the pre-tensioning effect, as previewed on Chapter 3. Analogously, the natural periods vary largely. The results from Table 8 show that the methodology herein proposed can indeed be used as a design tool, once it facilitates the calculation of a first estimative of natural periods for different design conditions.

Other important benefit of the methodology is that it allows us not only to easily evaluate the values of the mooring system stiffness at the trivial position, but also to map the values of the stiffness coefficients for different positions of the platform. As an example of this application, it is possible to vary the offset of the platform $r_{x}$ and $r_{y}$ within the interval of $\pm 20 \%$ of the radius from platform center to fairleads $R_{f}$ - i.e. $r_{x}^{*}=r_{y}^{*}=\left[-20 \% R_{f} ; 20 \% R_{f}\right]$, being $\left(r_{x}^{*}, r_{y}^{*}\right)=\left(r_{x} / R_{f}, r_{y} / R_{f}\right)$ - keeping the other generalized coordinate equal to zero. In other words, one can evaluate the stiffness matrix for $\mathbf{q}=\left[\begin{array}{llllll}r_{x} & r_{y} & 0 & 0 & 0 & 0\end{array}\right]$. Figures 41 to 43 exhibit these maps, presenting the partitions $\mathbb{K}_{T T}, \mathbb{K}_{T R}$ and $\mathbb{K}_{R R}$, considering the design pre-tensioning case. Notice that due to the symmetry of the stiffness matrix, only the upper-triangular matrix is shown - then $\mathbb{K}_{T R}=\mathbb{K}_{R T}$. It is notable that this way of presenting the results is also innovative.

One should interpret these colored maps as follows: Suppose that a constant external excitation changes the position from the unloaded equilibrium. Obviously, the stiffness matrix due to the mooring system is no longer that calculated at the initial position. This justifies the use of the presented methodology, allowing the designer to evaluate the stiffness at any point in an easy way. Some stability studies may also be done from the maps, but this is left for a further work.

From these maps, it is possible to see that stiffness coefficients largely vary, even if the offset is within operational limits. It is peculiar that some degrees of freedom that are not coupled at the trivial position, become coupled at certain offsets. For example, notice the stiffness coefficient $K_{12}$ for an offset along the coordinate $r_{y}$ : a coupling between the motions in directions $r_{x}$ and $r_{y}$ can be observed. This demonstrates the high nonlinear characteristic of the mooring system and justifies the use of an analytical model for rapidly evaluating the stiffness at that position - and not considering only the stiffness matrix at the trivial equilibrium position.

Some symmetric and anti-symmetric patterns are also possible to be seen. For instance, notice the symmetry of the elements in the diagonal with respect to the displacement $r_{y}$, as expected following the symmetry of the mooring system at the trivial position. Considering the planar DoFs, i.e. $r_{x}, r_{y}$ and $\psi$, the symmetric-anti-symmetric pattern is even more pronounced. $K_{12}$ is anti-symmetric with respect to $r_{y}$ while $K_{26}$ is symmetric to both, $r_{x}$ and $r_{y}$. 
Figure 41 - Partition $\mathbb{K}_{T T}$ of the stiffness matrix as function of the offset. Unities: $\mathrm{kN} / \mathrm{m}$.
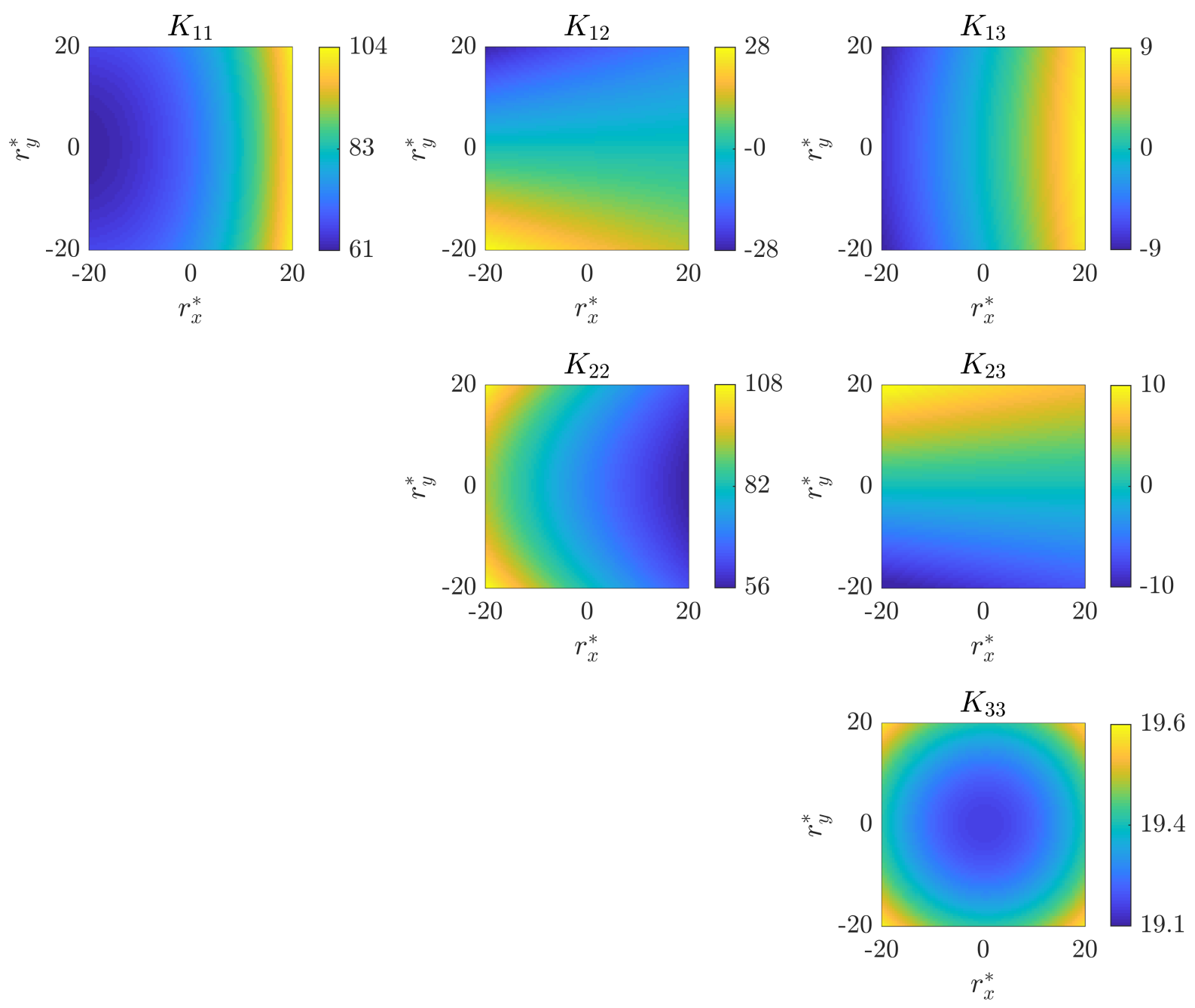

Source: The Author 
Figure 42 - Partitions $\mathbb{K}_{T R}$ and $\mathbb{K}_{R T}$ of the stiffness matrix as function of the offset. Unities: $\mathrm{kN} / \mathrm{rad}\left(\mathbb{K}_{T R}\right)$ or $\mathrm{kNm} / \mathrm{m}\left(\mathbb{K}_{R T}\right)$.
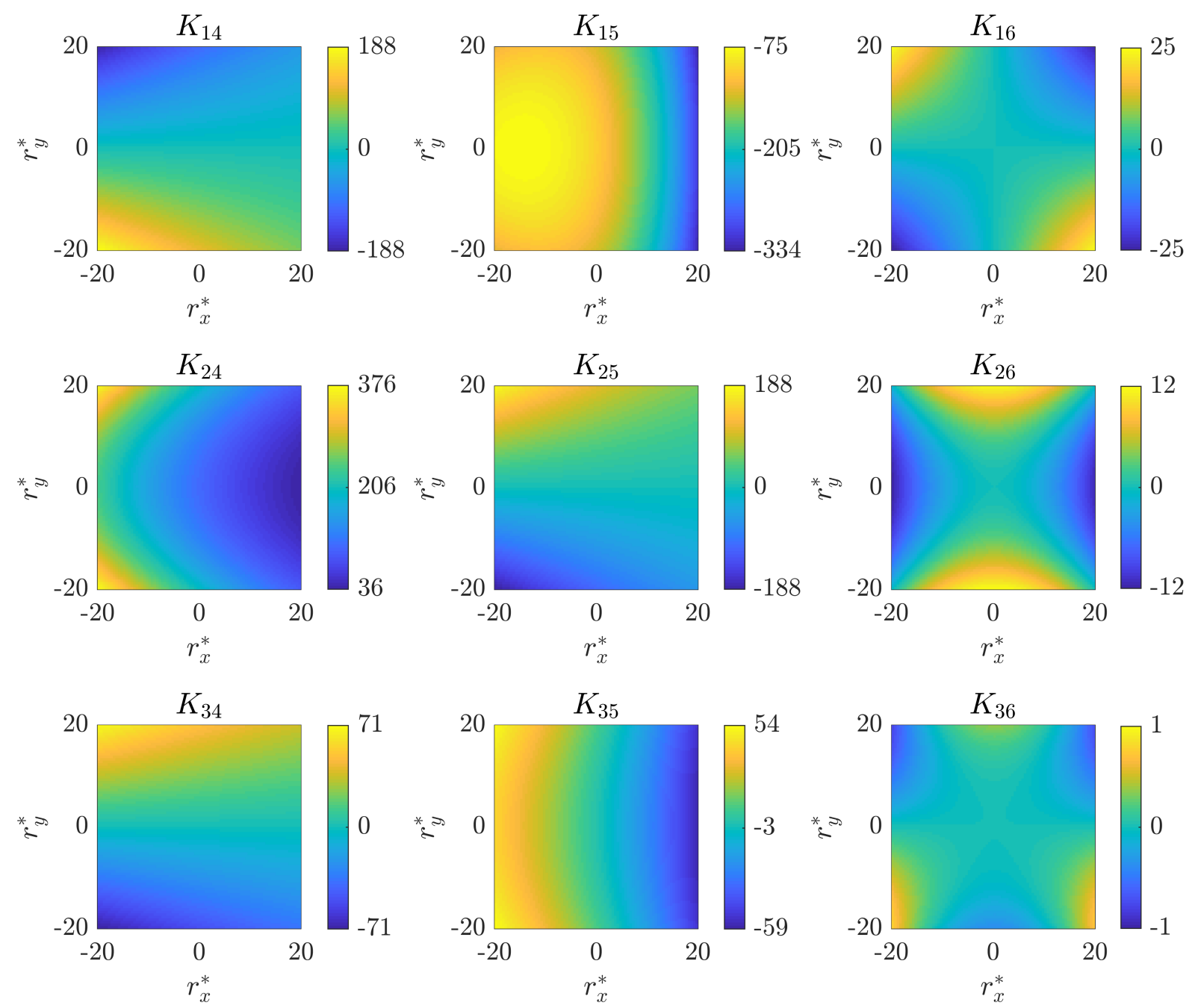

Source: The Author 
Figure 43 - Partition $\mathbb{K}_{R R}$ of the stiffness matrix as function of the offset. Unities: $\mathrm{kNm} / \mathrm{rad}$.
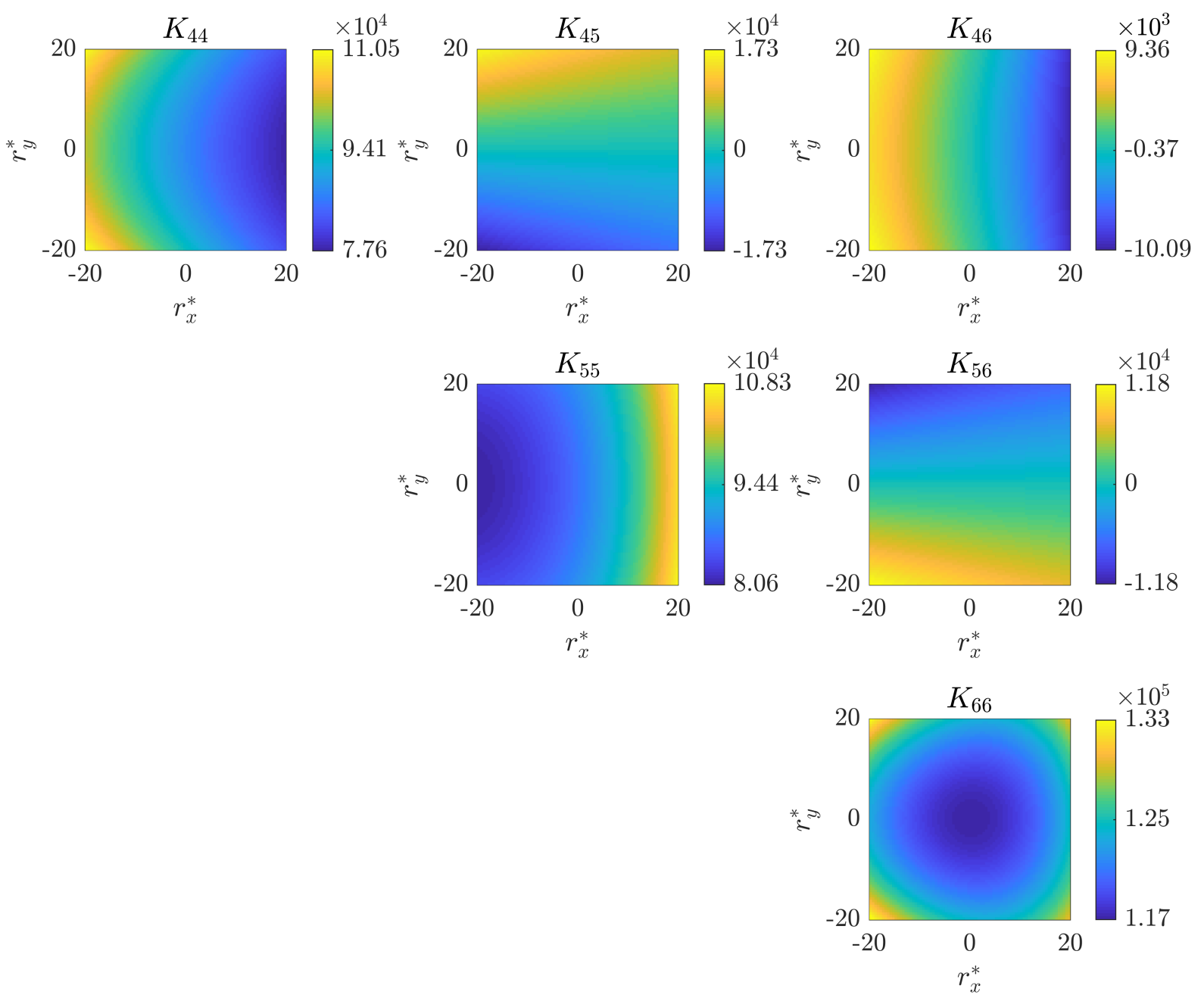

Source: The Author 
Again, the mean position of the vessel may influence the natural periods of oscillations. In this context, the linear eigenvalue problem is solved within the same intervals and the corresponding colored maps are presented in Figure 44, considering only the design pre-tensioning case. However, Figure 45 rearranges the natural periods considering the predominant direction of the motion: surge, sway and yaw ${ }^{4}$.

Figure 44 - The OC4-DeepCwind natural periods as function of the offset. Unity: s.

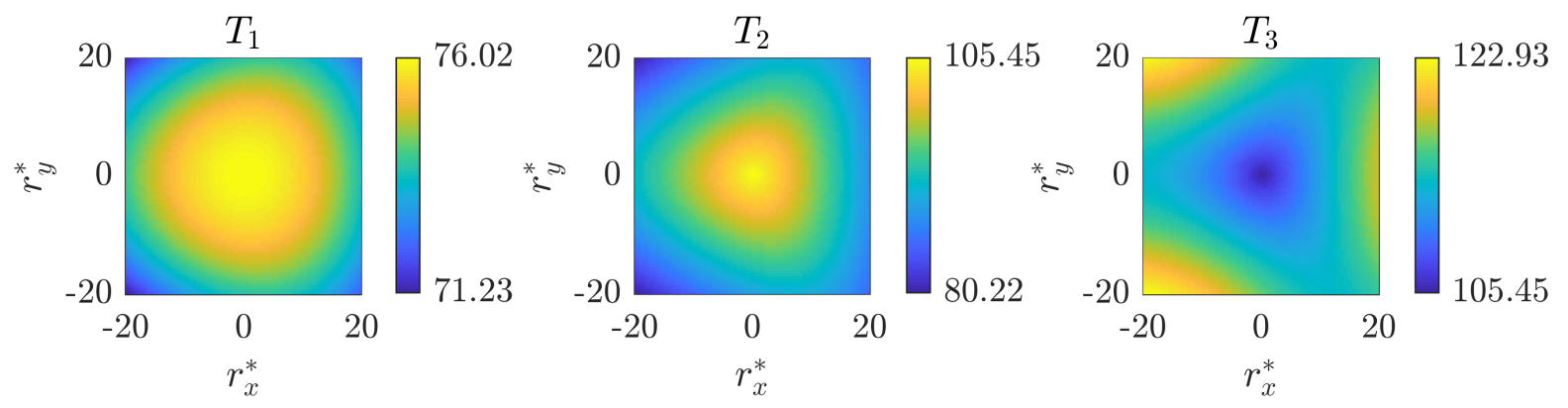

Source: The Author

Figure 45 - The OC4-DeepCwind natural periods as function of the offset: preponderant directions. Unity: s.

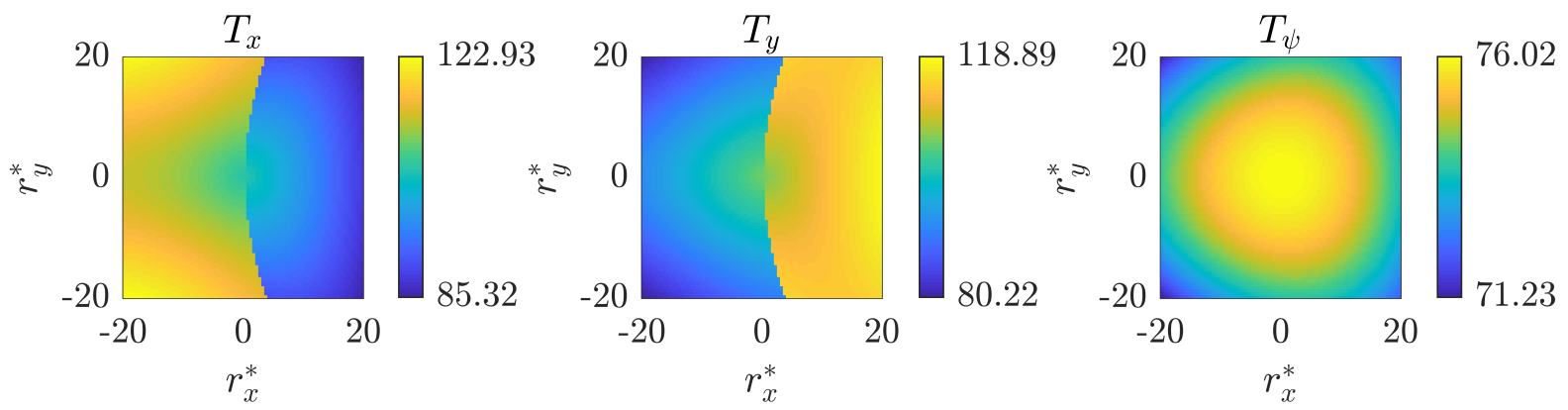

Source: The Author

It is noticeable that the periods largely vary due to vessel offset. This demonstrates once more the applicability of the presented tool. Once the designer usually aims to desynchronize the natural periods of motion with external forces, these maps indicates in an uncomplicated way the natural periods values for a shifted offset position. In turn, the maps from Figure 45 present an interesting pattern. Depending on the shifted position, the mode with higher period changes from being a surge-predominant to a sway-predominant. Regard that the yaw-predominant mode is exactly mode 1. Again, these maps can be of importance for the design of mooring systems.

$4 \quad$ Notice that at the drifted positions, motions along directions $r_{x}$ and $r_{y}$ can still be confound with the ocean engineering motions "surge" and "sway", as the rotation remains $\psi=0^{\circ}$. 
The shifted position can also lead to a coupling of the motions on the vibration modes. Figure 46 brings the natural periods and modes for the trivial and for four shifted positions: $\left(r_{x}^{*}, r_{y}^{*}\right)=( \pm 0.20, \pm 0.20)$. Notice that the aforementioned coupling is even more pronounced for horizontal motions. For example, modes 2 and 3 were initially pure sway and surge modes, but when presented for the shifted position there is a coupling between them. In other words, it is notable a composition of modes. In turn, mode 1, that for the trivial was an only yaw mode, became a surge-sway-yaw mode for all shifted positions presented. The symmetry of the mooring system arrangement with respect to $O x$ axis is reproduced on the periods, but it produces an antisymmetric pattern for the modes (e.g., for shifted positions $\left(r_{x}^{*}, r_{y}^{*}\right)=(0.20,0.20)$ and $\left(r_{x}^{*}, r_{y}^{*}\right)=(0.20,-0.20)$, notice that mode 2 and mode 3 are mirrored with respect to surge and sway motions, respectively.

The symmetric / antisymmetric patterns can not be seen with respect to $O y$ axis. Finally, it is notable that the mode with the higher period (mode 3) can be either a surge-predominant or a sway-predominant mode, depending on the shifted position with respect to $O y$ axis (e.g., mode 3 for shifted positions $\left(r_{x}^{*}, r_{y}^{*}\right)=(0.20,0.20)$ and $\left(r_{x}^{*}, r_{y}^{*}\right)=$ $(-0.20,0.20)$ are a sway-predominant and surge-predominant modes, respectively). This is the same conclusion obtained from Figure 45.

Figure 46 - The OC4-DeepCwind modes of oscillation for the trivial and shifted equilibrium positions, corresponding to natural periods $T_{j}, j=1,2,3$.
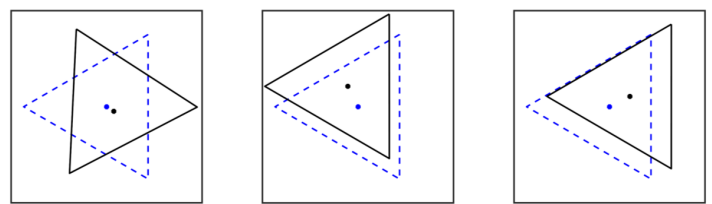

$\left(r_{x}^{*}, r_{y}^{*}\right)=(-0.20,0.20)$
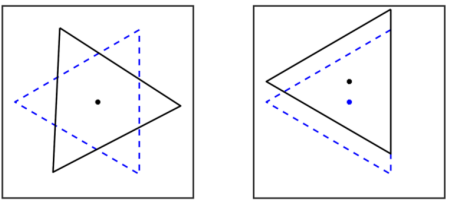

$\left(r_{x}^{*}, r_{y}^{*}\right)=(0,0)$
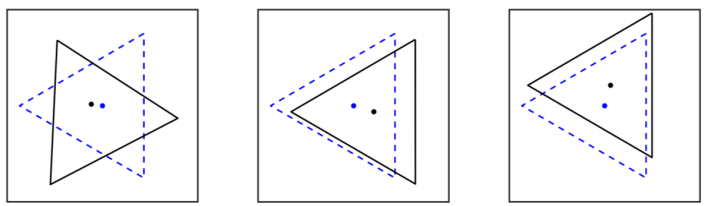

$\left(r_{x}^{*}, r_{y}^{*}\right)=(0.20,0.20)$
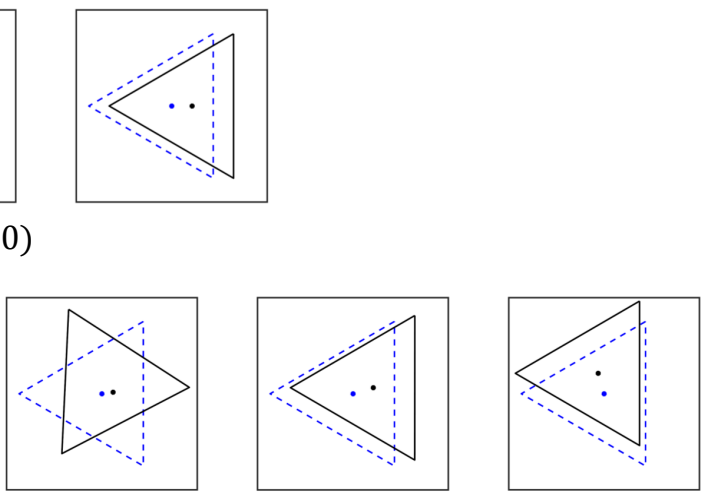

$\left(r_{x}^{*}, r_{y}^{*}\right)=(0.20,-0.20)$

$\left(r_{x}^{*}, r_{y}^{*}\right)=(-0.20,-0.20)$

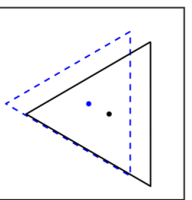

Source: The Author

Besides the vessel offset, its heading also presents an interesting influence on the stiffness coefficients. In fact, for the same case represented in Figures 41 to 43, let us 
suppose that the mean yaw position, no longer the trivial one, but at an initial heading angle $\bar{\psi}=5^{\circ}$, with all other generalized coordinates null. In other words, let us map the stiffness coefficients for $\mathbf{q}=\left[\begin{array}{llllll}r_{x} & r_{y} & 0 & 0 & 0 & \bar{\psi}\end{array}\right]$. Figures 47 to 49 bring these results, again presenting all the partitions.

Notice that the terms from the first partition, $\mathbb{K}_{T T}$, remain almost the same. In other words, the effect of an initial heading angle does not impact drastically the stiffness coefficients for the translation-only motions. This could be previewed just by considering the stiffness coefficients from Equations 3.30 to $3.32,3.36$ to 3.38 and 3.32 to 3.44 .

However, the coefficients from partition $\mathbb{K}_{T R}$ are the most influenced by the heading condition. It is interesting that some symmetric/antisymmetric patterns from Figure 42 are broken in Figure 48. For instance, coefficient $K_{15}$ has its point of major stiffness shifted from $r_{y}^{*}=0$ to somewhere near to $r_{y}^{*}=10 \%$. Indeed, the whole map seems to rotate. The heading angle degenerates the double antisymmetric pattern from coefficient $K_{16}$, which presents an antisymmetric pattern with respect to $r_{x}^{*}$ only, and symmetry with respect to $r_{y}^{*}$. Almost similarly, coefficient $K_{26}$ has its double symmetry degenerated to an antisymmetry with respect to $r_{y}^{*}$, only. In both cases $K_{16}$ and $K_{26}$, the stiffness greatly increased. Coefficient $K_{14}$ is no longer null at the $\left(r_{x}^{*}, r_{y}^{*}\right)=(0,0)$ and the whole map is reduced by $\sim 80 \mathrm{kN} / \mathrm{rad}$, keeping the same format. It is interesting to notice that coefficient $K_{36}$, initially null, presents a significant gain of stiffness. In other words, $\bar{\psi}$ plays an important role in the coupling $r_{z}-\psi$. The other coefficients $\left(K_{24}, K_{34}\right.$ and $\left.K_{35}\right)$ slightly vary.

Finally, coefficients from partition $\mathbb{K}_{R R}$ are also influenced by the initial heading angle. The effect is in order to increase the stiffness of the terms from the mean diagonal, mainly. This can be seen as a gain of stiffness in rotation motions of the vessel. 
Figure 47 - Partition $\mathbb{K}_{T T}$ of the stiffness matrix as function of the offset. Heading $=$ $\bar{\psi}=5^{\circ}$. Unities: $\mathrm{kN} / \mathrm{m}$.
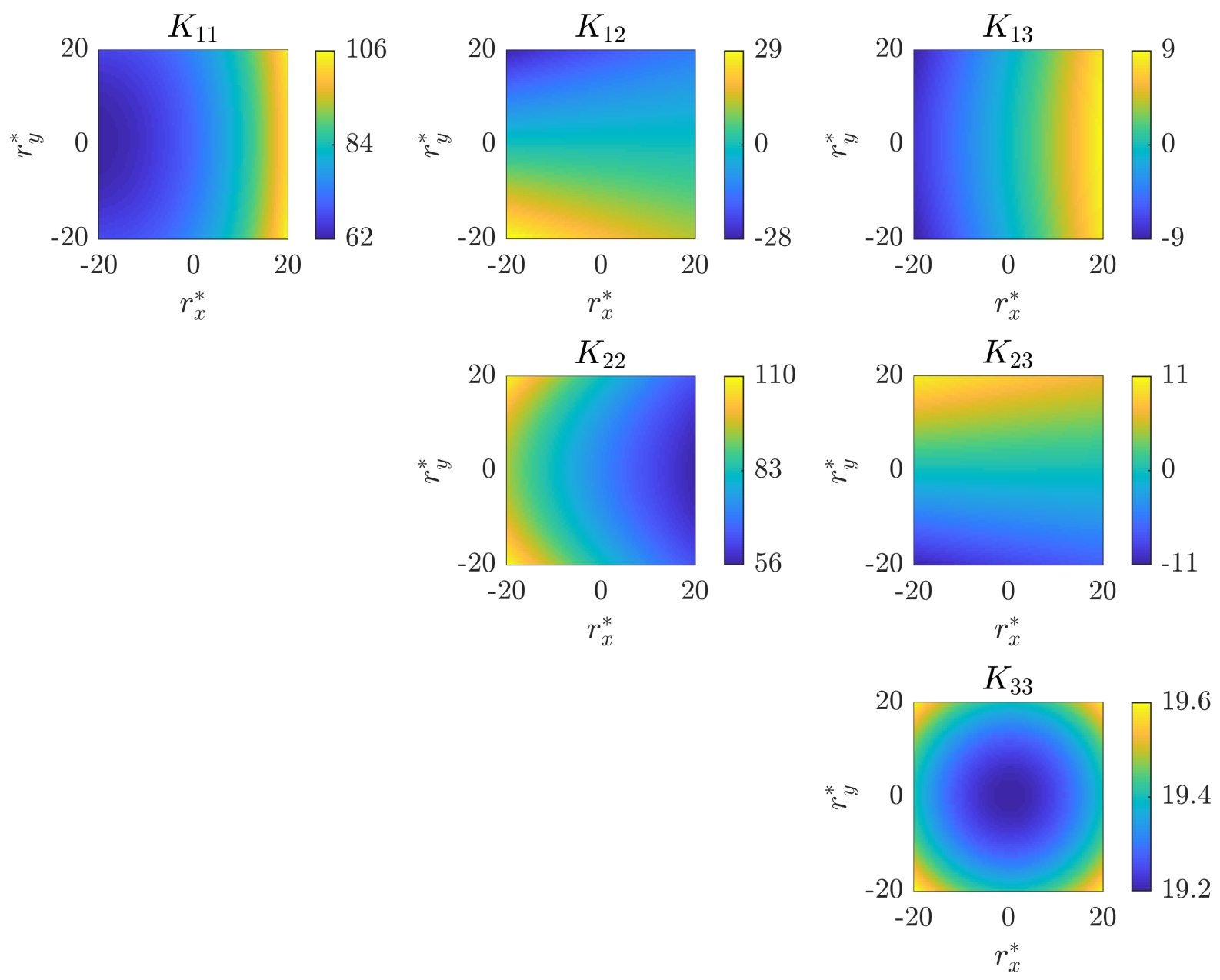

Source: The Author 
Figure 48 - Partitions $\mathbb{K}_{T R}$ and $\mathbb{K}_{R T}$ of the stiffness matrix as function of the offset. Heading $=\bar{\psi}=5^{\circ}$. Unities: $\mathrm{kN} / \mathrm{rad}\left(\mathbb{K}_{T R}\right)$ or $\mathrm{kNm} / \mathrm{m}\left(\mathbb{K}_{R T}\right)$.
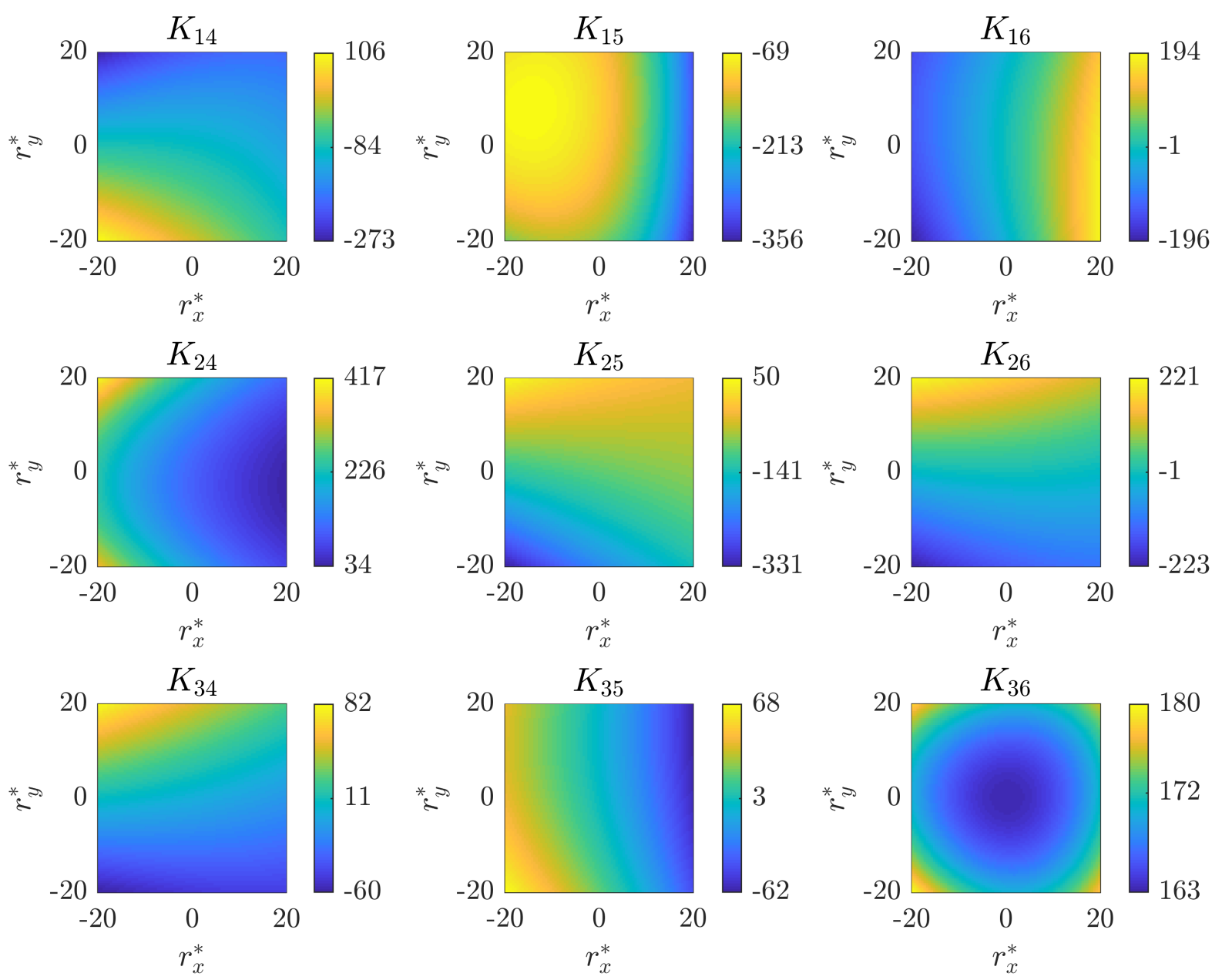

Source: The Author 
Figure 49 - Partition $\mathbb{K}_{R R}$ of the stiffness matrix as function of the offset. Heading = $\bar{\psi}=5^{\circ}$. Unities: $\mathrm{kNm} / \mathrm{rad}$.
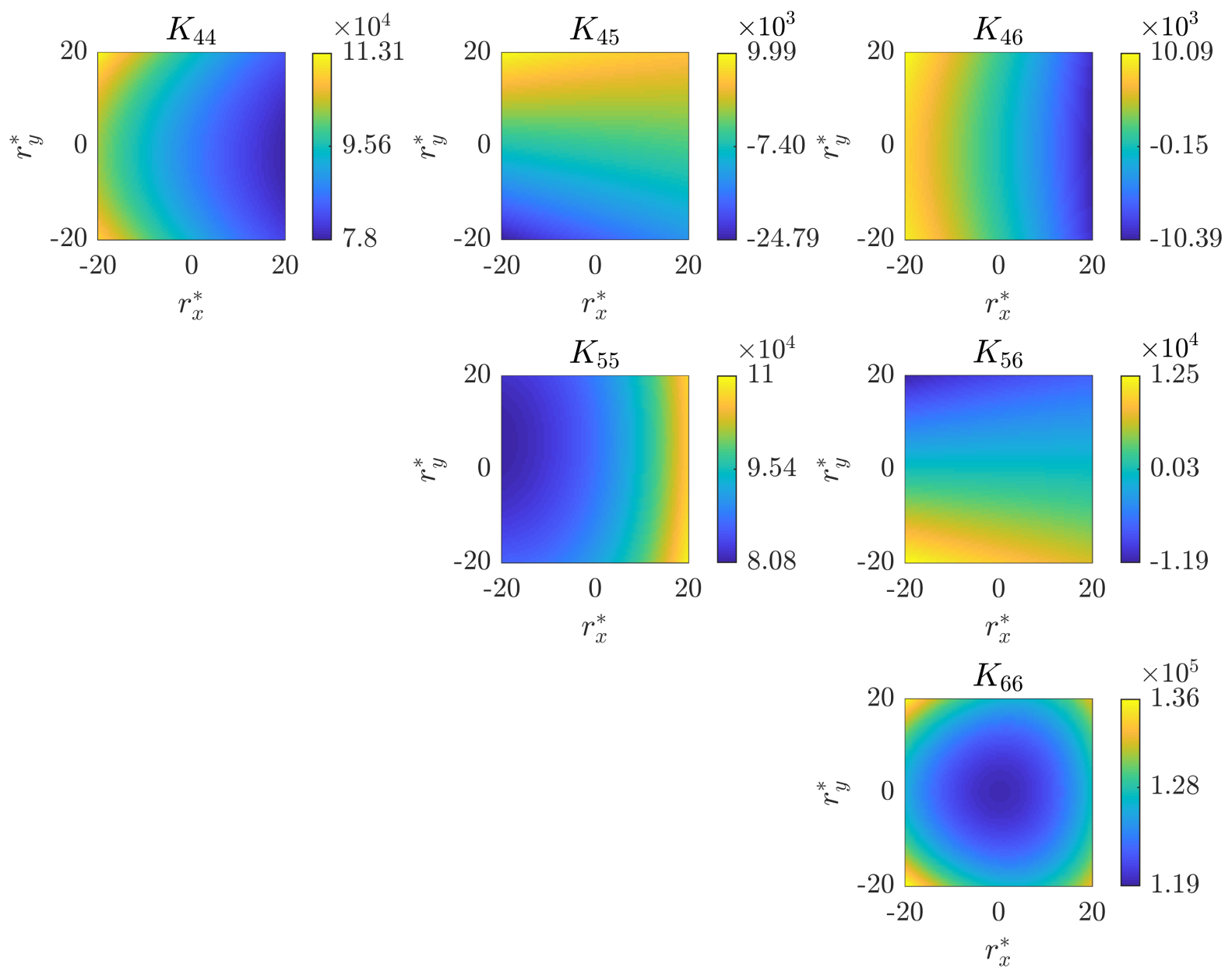

Source: The Author 
Now, the stiffness coefficients and natural periods can be evaluated as function of both the pre-tensioning and the vessel offset. Figures 50 to 58 present the partitions of the stiffness matrix for the three pre-tensioning cases. The color scales are the same in all cases for each stiffness coefficient. Notice that Figures 53 to 55 are the same as Figures 41 to 43 presented earlier, but with a different color scale in order to allow the reader to better compare the effects of the pre-tensioning on the stiffness coefficient. In turn, Figure 59 presents the periods as function of the mean offset position also for the three pre-tensioning cases.

Again, it is significant that the pre-tensioning plays an important role on the stiffness of the system. Notice that all the coefficients of the main diagonal $\left(K_{j j}, j=1, . .6\right)$ are strongly affected by the pre-tensioning variation. The pre-tensioning increase reflects in an increase of those stiffness coefficients. Additionally, the surge-pitch and sway-roll ${ }^{5}$ coefficients (i. e. $K_{15}$ and $K_{24}$, respectively) also vary with the pre-tensioning. However, while the $K_{24}$ increases, $K_{15}$ decreases. However, one may say that $K_{15}$ increases in absolute value. In other words, the non-null coefficients at the trivial position are the most sensitive to pre-tensioning variation and they experiment an absolute increase of stiffness. Once more, these colored maps demonstrate the importance of a proper generic analytical tool to evaluate stiffness coefficients.

In turn, as the increase of pre-tension results in an increase of stiffness of the system, it is natural to expect that the natural periods reduce, as presented in Figure 59. A practical result can be extracted from this: changing the total length of the line during a mooring operation can be an easy alternative in order to desynchronize the system natural periods from those of the external forces. Also, the colored maps from Figure 59 can be employed during the early stages of the project to investigate the range of the natural periods for different design configurations. Thus, the presented methodology can be an useful tool not only to evaluate the stiffness and natural periods for different offset conditions but also during design and operation of moored systems.

5 Notice that for the present study, the rotation angles remain the same, and $r_{x}, r_{y}$ and $r_{z}$ motions can be confounded with the ocean engineering classical surge, sway and heave motions, respectively 
Figure 50 - Partition $\mathbb{K}_{T T}$ of the stiffness matrix as function of the offset. Low pretensioning case. Pre-tensioning ratio: $f^{*}=0.9$. Unities: $\mathrm{kN} / \mathrm{m}$.
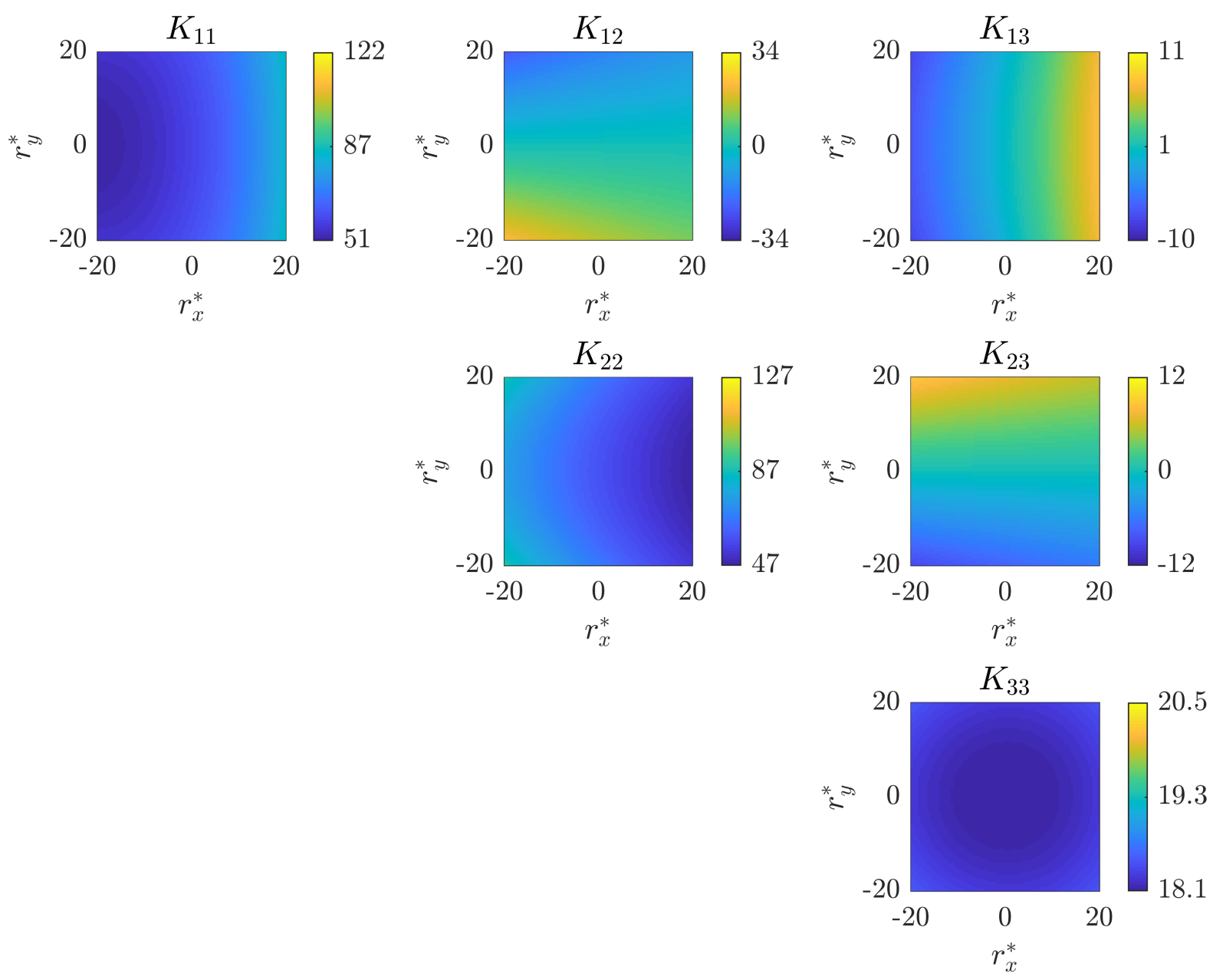

Source: The Author 
Figure 51 - Partitions $\mathbb{K}_{T R}$ and $\mathbb{K}_{R T}$ of the stiffness matrix as function of the offset. Low pre-tensioning case. Pre-tensioning ratio: $f^{*}=0.9$. Unities: $\mathrm{kN} / \mathrm{rad}\left(\mathbb{K}_{T R}\right)$ or $\mathrm{kNm} / \mathrm{m}\left(\mathbb{K}_{R T}\right)$.
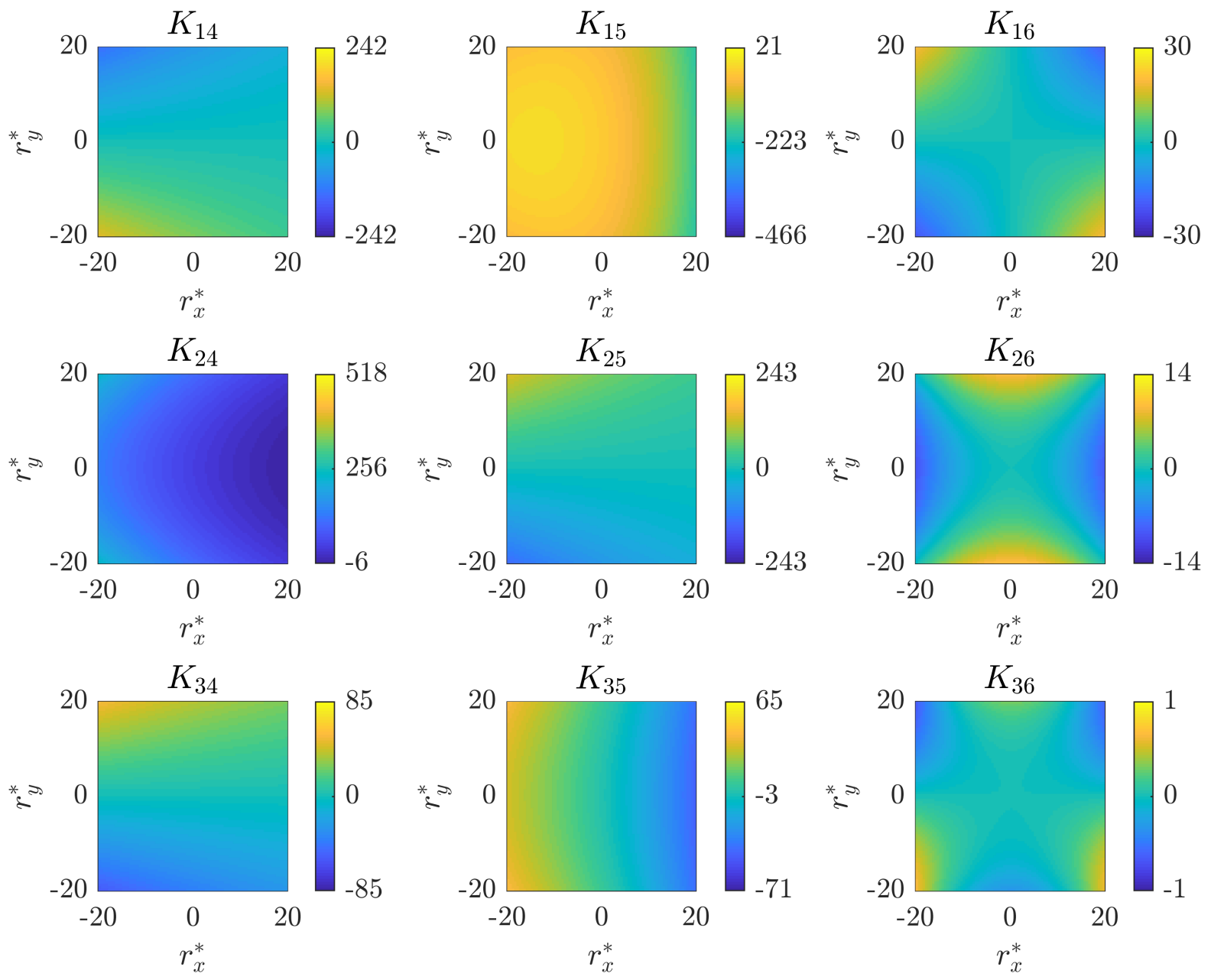

Source: The Author 
Figure 52 - Partition $\mathbb{K}_{R R}$ of the stiffness matrix as function of the offset. Low pretensioning case. Pre-tensioning ratio: $f^{*}=0.9$. Unities: $\mathrm{kNm} / \mathrm{rad}$.

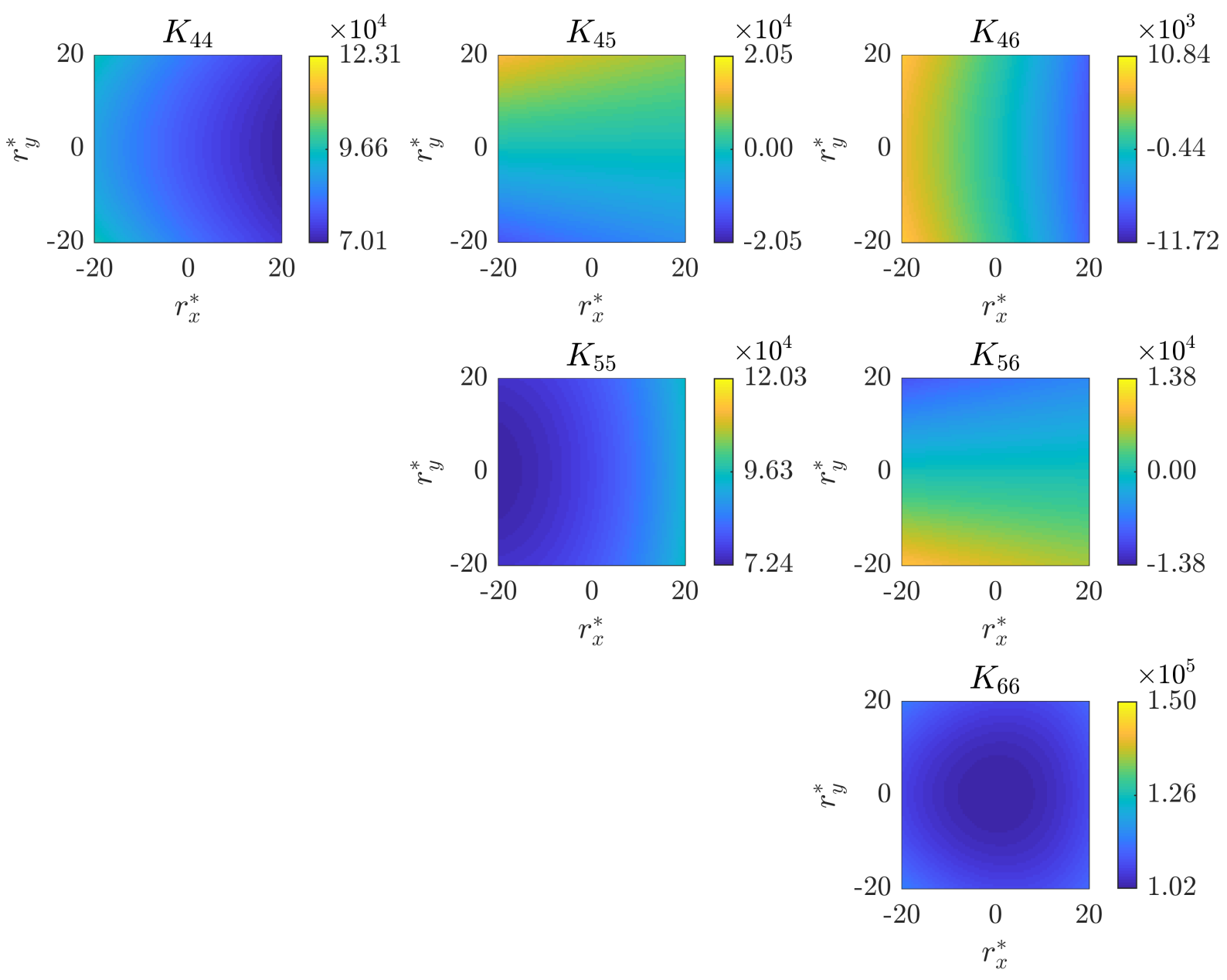

Source: The Author 
Figure 53 - Partition $\mathbb{K}_{T T}$ of the stiffness matrix as function of the offset. Design pretensioning case. Pre-tensioning ratio: $f^{*}=1.0$. Unities: $\mathrm{kN} / \mathrm{m}$.
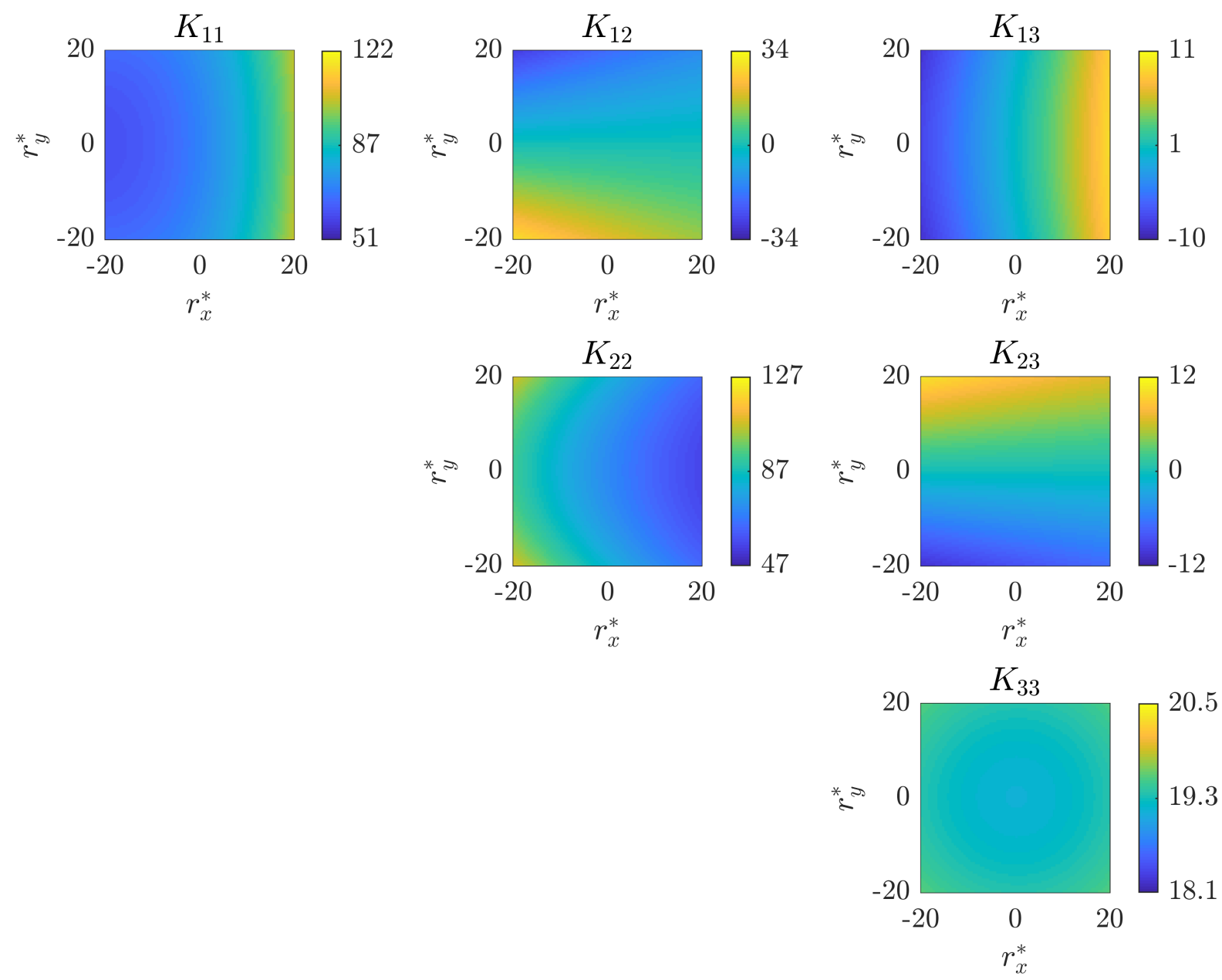

Source: The Author 
Figure 54 - Partitions $\mathbb{K}_{T R}$ and $\mathbb{K}_{R T}$ of the stiffness matrix as function of the offset. Design pre-tensioning case. Pre-tensioning ratio: $f^{*}=1$.0. Unities: $\mathrm{kN} / \mathrm{rad}\left(\mathbb{K}_{T R}\right)$ or $\mathrm{kNm} / \mathrm{m}\left(\mathbb{K}_{R T}\right)$.
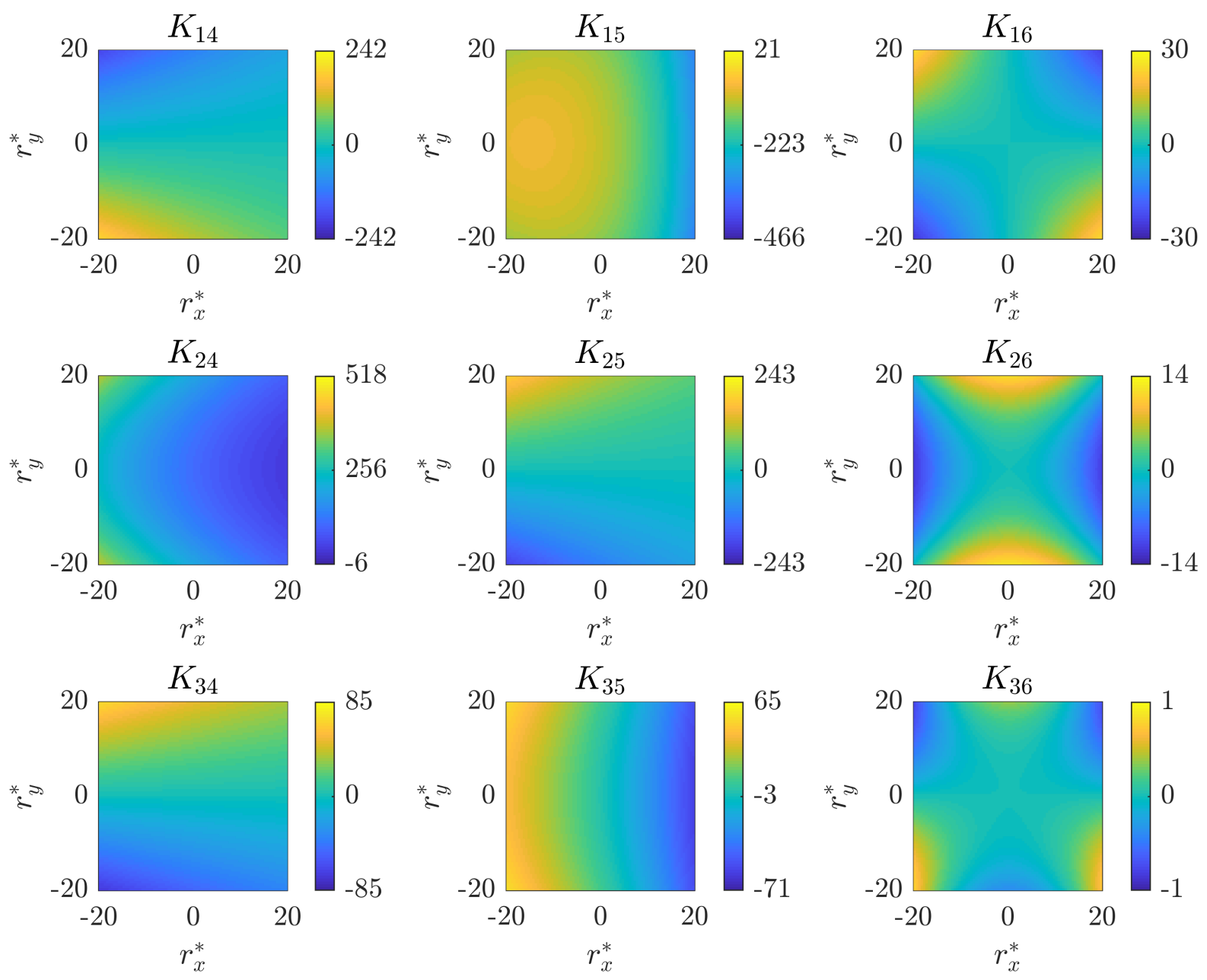

Source: The Author 
Figure 55 - Partition $\mathbb{K}_{R R}$ of the stiffness matrix as function of the offset. Design pretensioning case. Pre-tensioning ratio: $f^{*}=1.0$. Unities: $\mathrm{kNm} / \mathrm{rad}$.
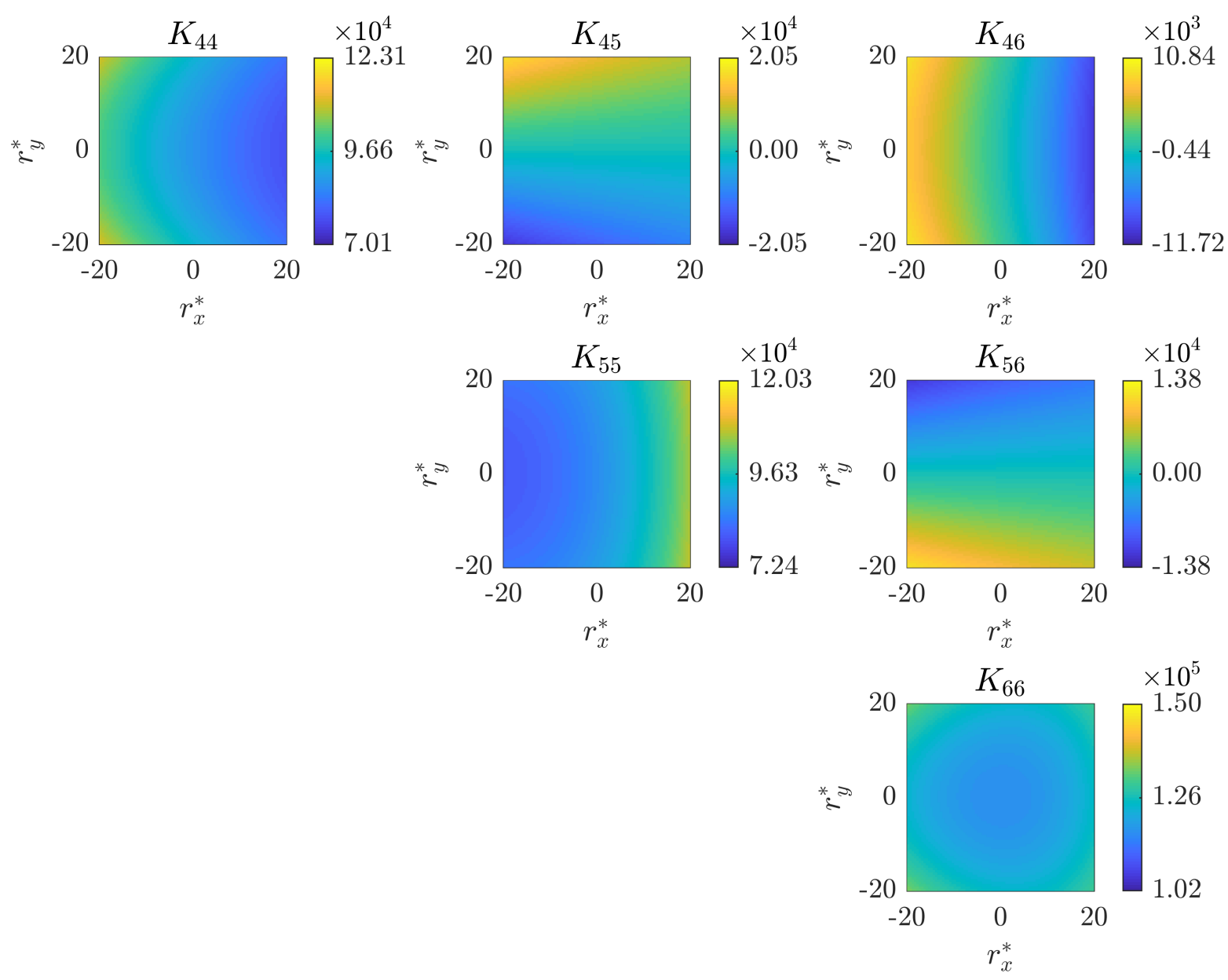

Source: The Author 
Figure 56 - Partition $\mathbb{K}_{T T}$ of the stiffness matrix as function of the offset. High pretensioning case. Pre-tensioning ratio: $f^{*}=1.1$. Unities: $\mathrm{kN} / \mathrm{m}$.
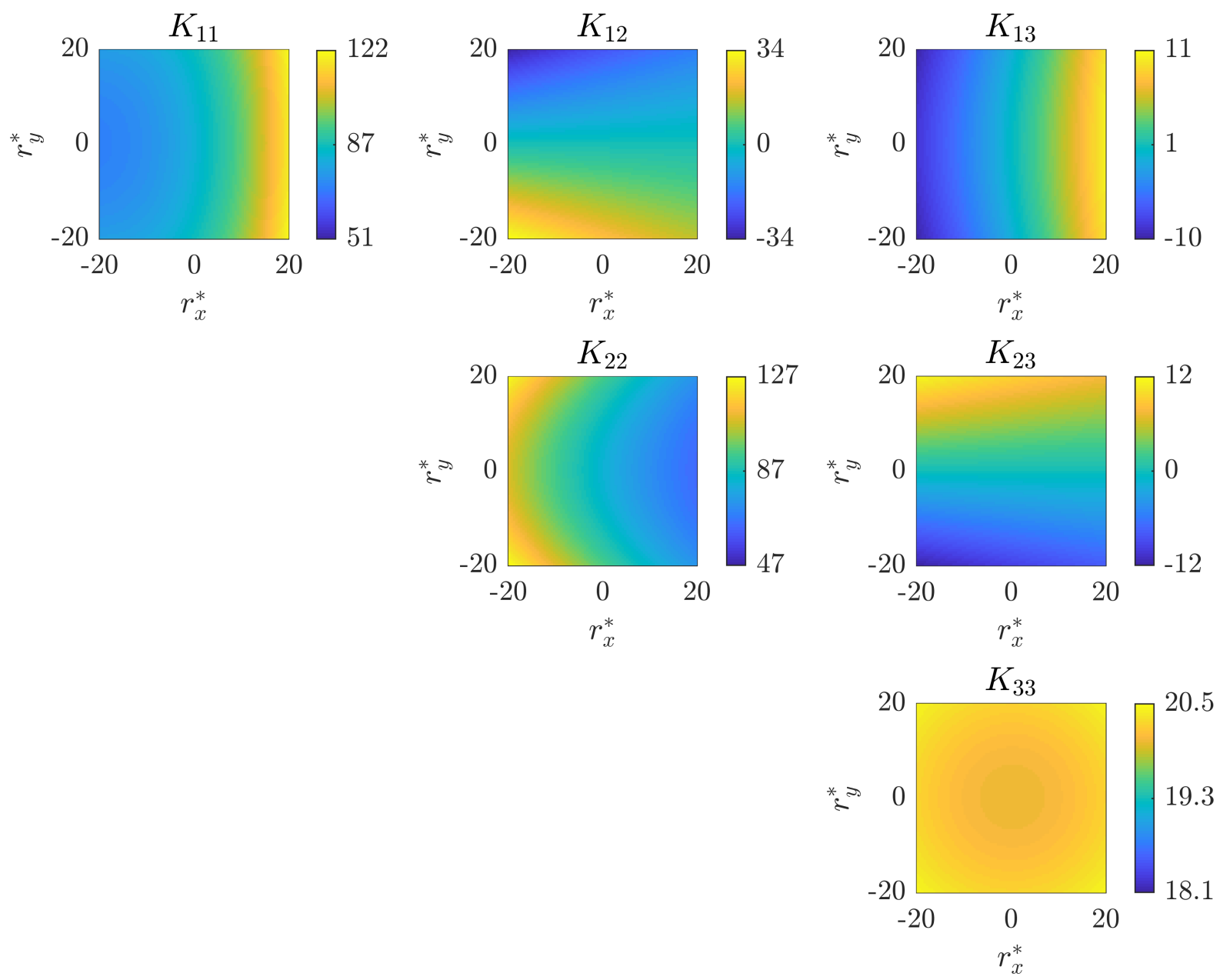

Source: The Author 
Figure 57 - Partitions $\mathbb{K}_{T R}$ and $\mathbb{K}_{R T}$ of the stiffness matrix as function of the offset. High pre-tensioning case. Pre-tensioning ratio: $f^{*}=1.1$. Unities: $\mathrm{kN} / \mathrm{rad}\left(\mathbb{K}_{T R}\right)$ or $\mathrm{kNm} / \mathrm{m}\left(\mathbb{K}_{R T}\right)$.
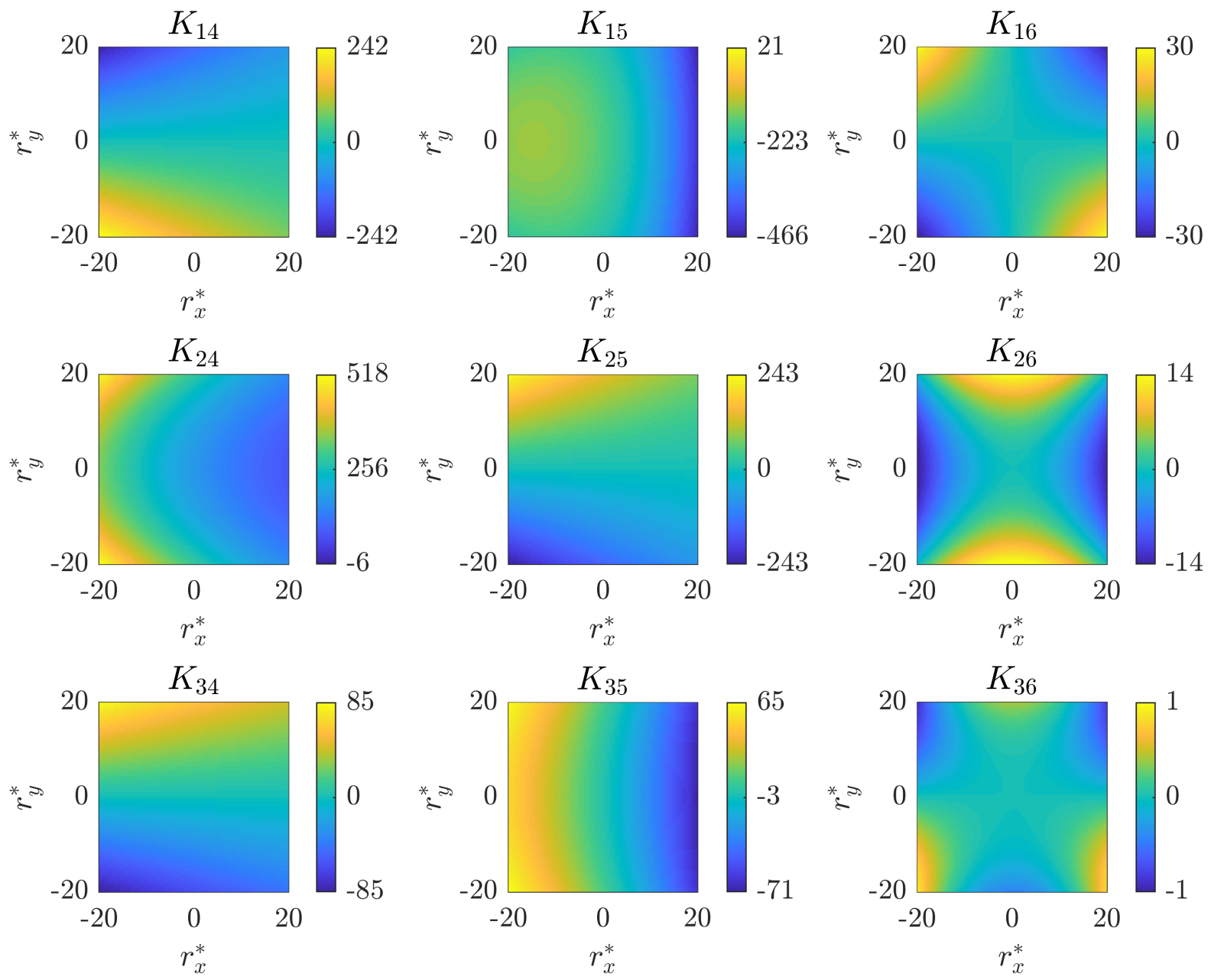

Source: The Author 
Figure 58 - Partition $\mathbb{K}_{R R}$ of the stiffness matrix as function of the offset. High pretensioning case. Pre-tensioning ratio: $f^{*}=1.1$. Unities: $\mathrm{kNm} / \mathrm{rad}$.
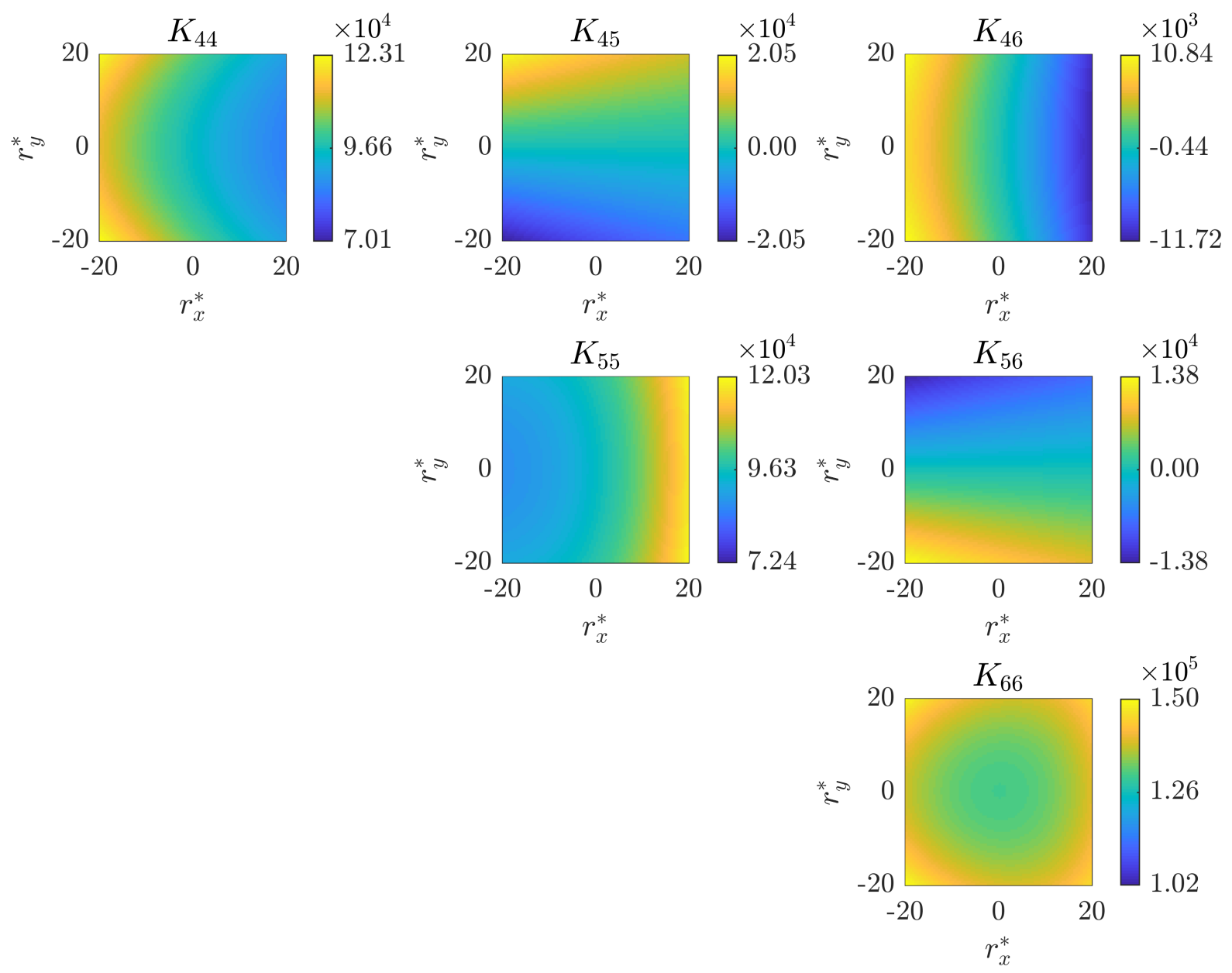

Source: The Author 
Figure 59 - The OC4-DeepCwind natural periods as function of the offset and the pretensioning. Unities: s.
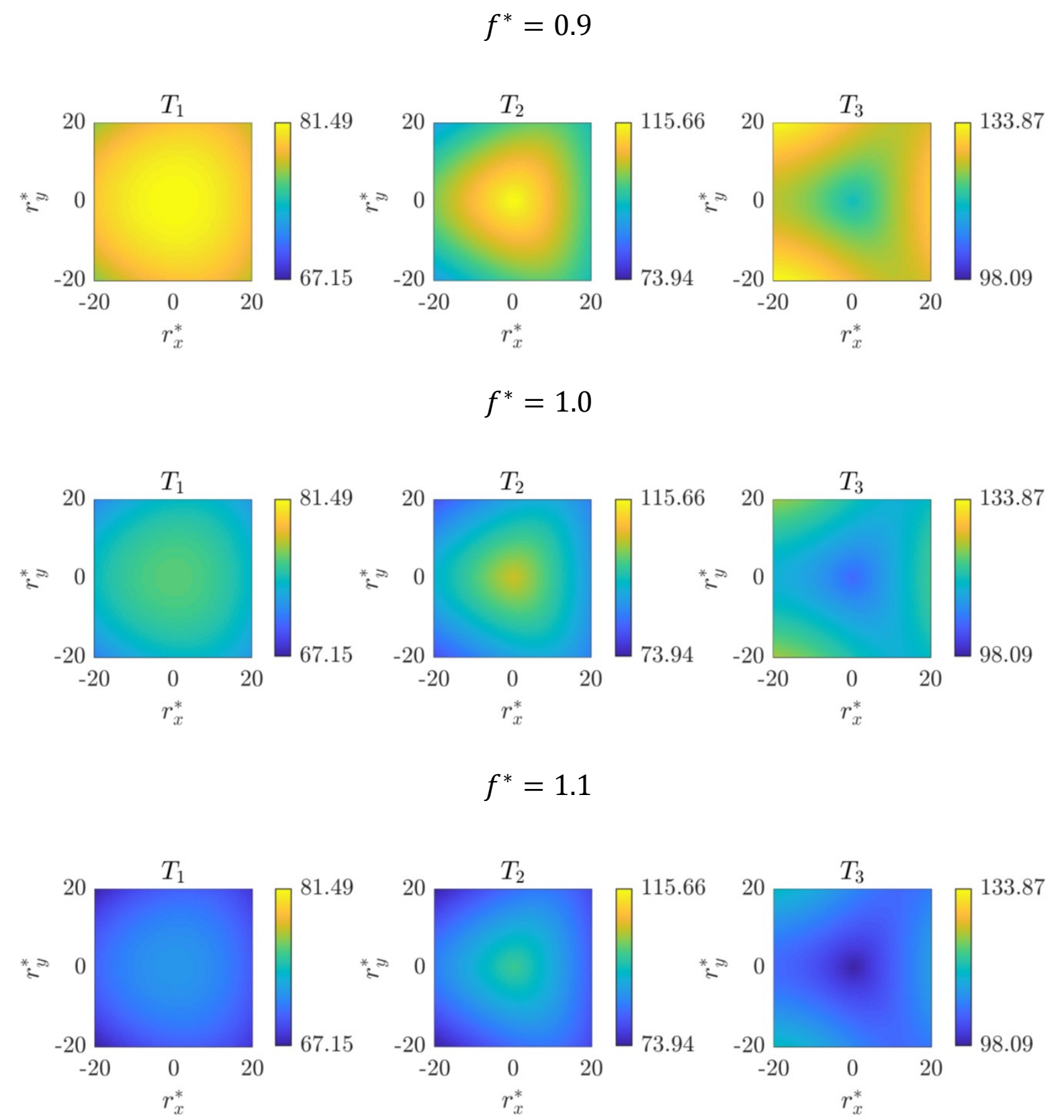

Source: The Author 


\subsection{Chain-wire-chain catenary mooring line}

An alternative mooring system is proposed for the OC4-DeepCwind platform. A $3 \times 2$ symmetric spread mooring system is evaluated. The the lines are spaced of $\beta=5^{\circ}$ inside the cluster. Figure 61(a) shows its top view. All six mooring lines are equal with a three-segment chain-wire-chain profile, as represented in Figure 61(b). Table 9 brings the details of the mooring system parameters. This system aims to give the same vertical restoring force as the original one, but with a smaller anchor radius. The parameters of the line were calculated using the estimation presented in Ma et al. (2019).

Figure 60 - The OC4-DeepCwind floating wind alternative mooring system: (a) Top view and (b) Chain-wire-chain mooring line profile.

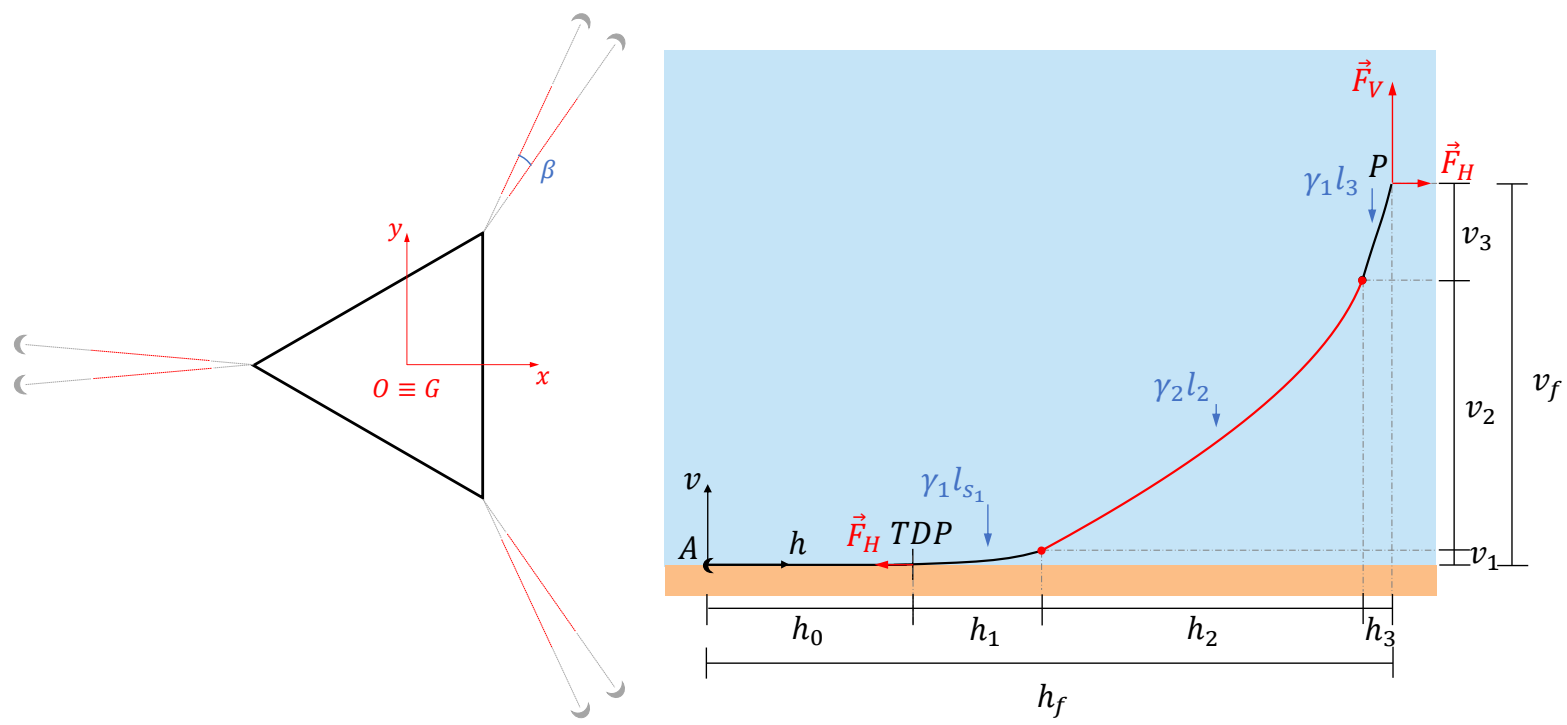

(a)

(b)

Source: The Author

The mooring line forces and stiffness should be evaluated as presented in Equations 3.106 and 3.107, taking $N=3$ and considering the case where a portion of the first segment rests at the seabed. Once more, the stiffness coefficients were numerically calculated using the Newton-Raphson algorithm, as presented in Appendix A. Table 10 brings these values for the trivial equilibrium point.

The analytical mooring system stiffness $\mathbb{K}_{\mathrm{ALT}}$ at trivial equilibrium position is calculated and presented in Equation 4.18 (values in $\mathrm{kN}, \mathrm{m}$ and $\mathrm{rad}$ ). Comparing it with the stiffness matrix $\mathbb{K}$ given by Equation 4.11, it is clear that the alternative mooring system is stiffer than the original one, since all diagonal terms increase. The off-diagonal terms present an inversion of signal, what could lead to changes on amplitudes and phases of coupled motions. The proposed arrangement was easily evaluated, demonstrating the 
Table 9 - The OC4-DeepCwind alternative mooring system parameters.

\begin{tabular}{lr}
\hline \hline Number of mooring lines & 6 \\
System type & $\begin{array}{r}\text { Spread system } \\
\text { Three-segment } \\
\text { Line profile }\end{array}$ \\
Line composition & $200 \mathrm{~m}$ \\
Water depth & $14 \mathrm{~m}$ \\
Fairlead depth & $596 \mathrm{~m}$ \\
Radius from center to anchors & $40.9 \mathrm{~m}$ \\
Radius from center to fairleads & $400 \mathrm{~m}$ \\
Segments 1 unstretched length & $227.35 \mathrm{~m}$ \\
Segment 2 unstretched length & $10 \mathrm{~m}$ \\
Segments 3 unstretched length & $111.85 \mathrm{~kg} / \mathrm{m}$ \\
Segments 1 and 3 mass per unit length & $7.01 \mathrm{~kg} / \mathrm{m}$ \\
Segment 2 mass per unit length & $76.6 \mathrm{~mm}$ \\
Segments 1 and 3 equivalent diameter & $40 \mathrm{~mm}$ \\
Segment 2 equivalent diameter & $592.6 \mathrm{MN}$ \\
Segments 1 and 3 Axial Stiffness & $141.9 \mathrm{MN}$ \\
Segment 2 Axial Stiffness
\end{tabular}

Source: The Author

Table 10 - The OC4-DeepCwind alternative mooring line forces and stiffness.

\begin{tabular}{lr}
\hline \hline Pre-tensioning & $6.25 \mathrm{E}+2 \mathrm{kN}$ \\
Horizontal force $\left(F_{H}\right)$ & $5.36 \mathrm{E}+2 \mathrm{kN}$ \\
Vertical force $\left(F_{V}\right)$ & $3.21 \mathrm{E}+2 \mathrm{kN}$ \\
Horizontal local stiffness $\left(k_{H H}\right)$ & $3.34 \mathrm{E}+1 \mathrm{kN} / \mathrm{m}$ \\
Vertical local stiffness $\left(k_{V V}\right)$ & $6.15 \mathrm{kN} / \mathrm{m}$ \\
Coupled local stiffness $\left(k_{H V}\right)$ & $1.26 \mathrm{E}+1 \mathrm{kN} / \mathrm{m}$ \\
Horizontal "string stiffness" $\left(\bar{k}_{H H}\right)$ & $0.90 \mathrm{kN} / \mathrm{m}$ \\
\hline \hline
\end{tabular}

Source: The Author

use of the methodology as a design tool allowing to compare different alternatives.

$$
\mathbb{K}=\left(\begin{array}{cccccc}
1.03 \mathrm{E}+2 & 0 & 0 & 0 & 1.10 \mathrm{E}+2 & 0 \\
0 & 1.03 \mathrm{E}+2 & 0 & -1.10 \mathrm{E}+2 & 0 & 0 \\
0 & 0 & 3.69 \mathrm{E}+1 & 0 & 0 & 0 \\
0 & -1.10 \mathrm{E}+2 & 0 & 1.00 \mathrm{E}+5 & 0 & 0 \\
1.10 \mathrm{E}+2 & 0 & 0 & 0 & 1.00 \mathrm{E}+5 & 0 \\
0 & 0 & 0 & 0 & 0 & 1.41 \mathrm{E}+5
\end{array}\right)
$$

Again, it is possible to calculate the natural periods of oscillation for motions which take place on the horizontal plane. Table 11 brings these periods together with a comparison to those from the original OC4-DeepCwind mooring concept. 
Table 11 - The OC4-DeepCwind natural periods of oscillation for the trivial position: comparison between original and alternative mooring systems. Unities: s.

\begin{tabular}{ccc}
\hline \hline & Original & Alternative \\
\hline$T_{1}$ & 76.02 & 69.39 \\
$T_{2}$ & 105.48 & 87.49 \\
$T_{3}$ & 105.48 & 87.49 \\
\hline \hline
\end{tabular}

Source: The Author

It is worthy of attention that the alternative mooring system herein proposed reduces natural periods. This could have been anticipated by considering that it is stiffer than the original one.

Finally, the stiffness coefficients and natural periods for the alternative mooring system can be mapped as functions of the mean offset position. Figures 61 to 61 show the partitions of the stiffness matrix for the same range as used before, $r_{x}^{*}=r_{y}^{*}=$ $\left[-20 \% R_{f} ; 20 \% R_{f}\right]$. In this sense, Figure 64 presents the natural periods as function of the position.

As the alternative mooring system is symmetric and similar to the original one (SMS with three equidistant fairleads), the colored maps' disposition are also similar to those presented in Figures 41 to 43. In other words, it is the same symmetric/antisymmetric patterns for stiffness coefficients in the new system. However, as the proposed mooring system is stiffer than the original one, the range of the coefficients in Figures 61 to 63 is larger than in Figures 41 to 43. Indeed, all coefficients except $K_{14}$ and $K_{25}$ present higher differences between extremum, for the offset limit proposed. An interesting result comes from coefficients $K_{15}$ and $K_{24}$. Once more, these coefficients present an inversion of signal, as the trivial position in Equation 4.18. It is notable that for some offset conditions both coefficients can present the same signal, although they have opposite signals at unloaded equilibrium position.

Similarly to what happened to the trivial equilibrium position, the natural periods for other offset mean positions for the alternative mooring system are lower than for the original one. However, differently to the colored maps for the natural periods from the OC4-DeepCwind system, presented in Figure 44, the ranges from the $T_{1}$ and $T_{2}$ are overlaid. Indeed, notice that in Figure 44 all three colored maps present different ranges, while in Figure 64 they are not. This can indicate that the preponderant motion in the modes are different for each position - or even the presence of truly coupled modes -, including for the first mode.

Once more, all these analyses could only be made with the help of an analytical closed formulation, a practical and easy tool. High-hierarchical nonlinear models are too 
time-consuming, which makes unfeasible to run such analyses. Thus, the analytical tool herein proposed shows to be of great value in different stages of any offshore project, from early design steps to operation. 
Figure 61 - Partition $\mathbb{K}_{T T}$ of the stiffness matrix as function of the offset. Alternative mooring system. Unities: $\mathrm{kN} / \mathrm{m}$.
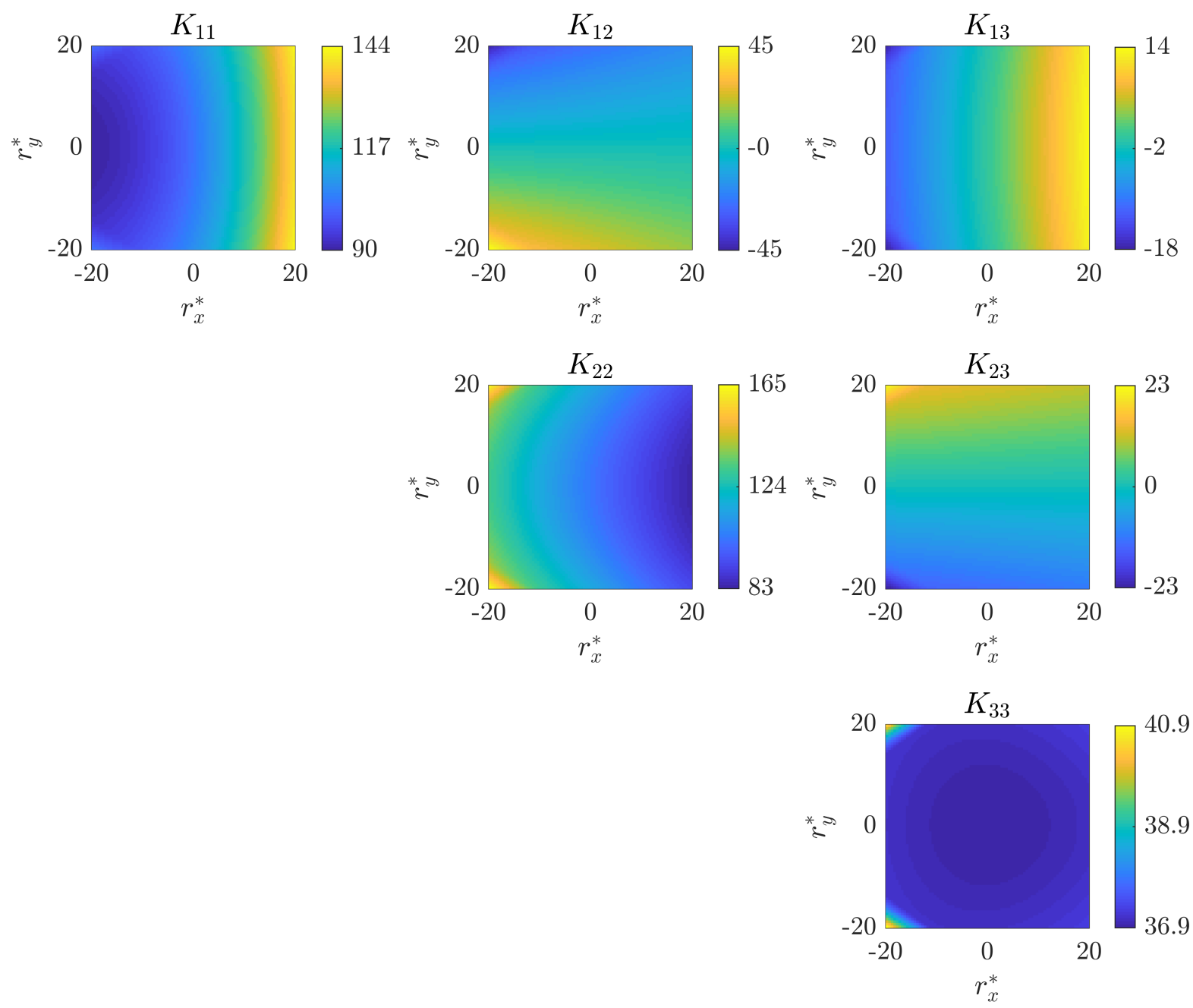

Source: The Author 
Figure 62 - Partitions $\mathbb{K}_{T R}$ and $\mathbb{K}_{R T}$ of the stiffness matrix as function of the offset. Alternative mooring system. Unities: $\mathrm{kN} / \mathrm{rad}\left(\mathbb{K}_{T R}\right)$ or $\mathrm{kNm} / \mathrm{m}\left(\mathbb{K}_{R T}\right)$.
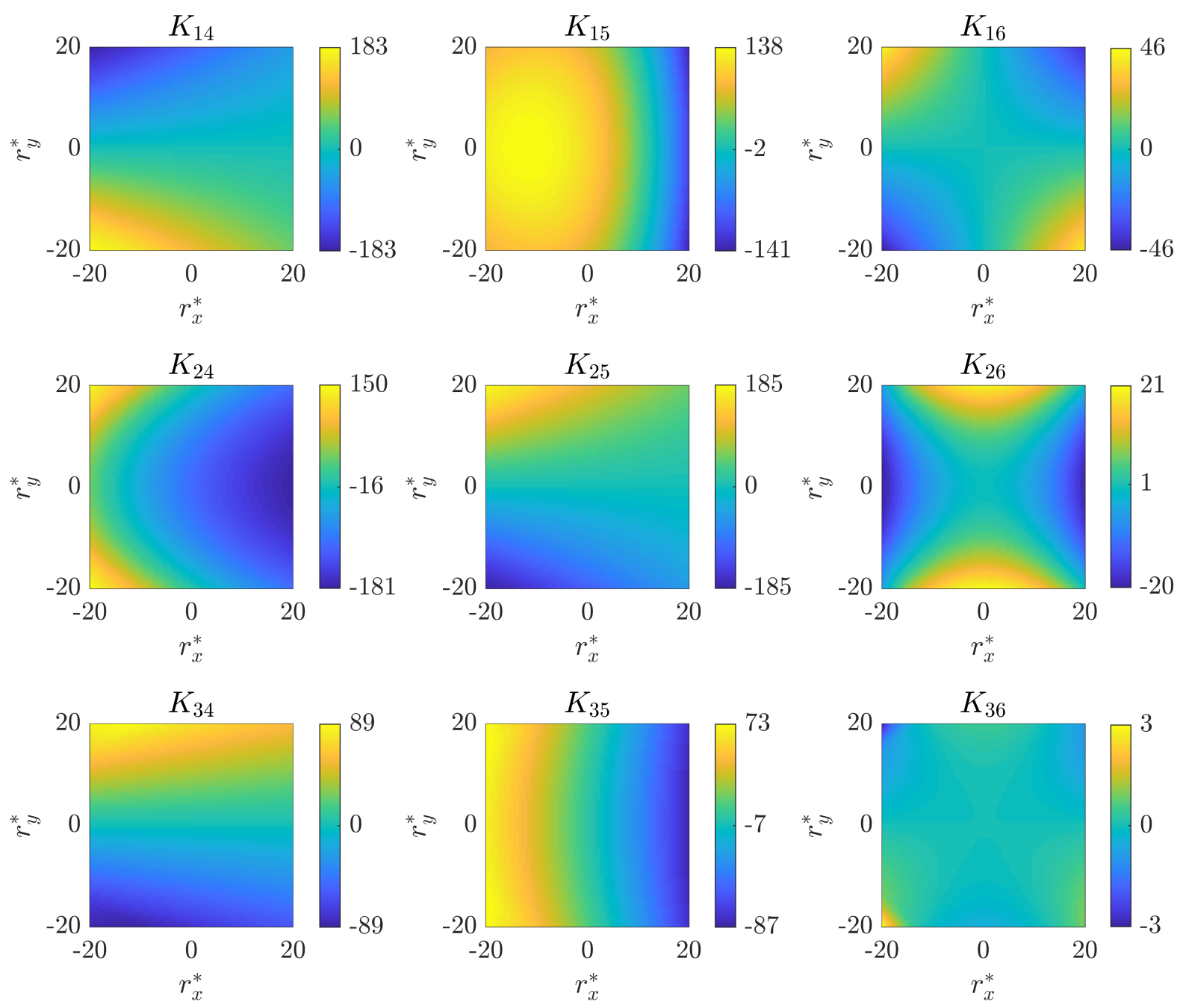

Source: The Author 
Figure 63 - Partition $\mathbb{K}_{R R}$ of the stiffness matrix as function of the offset. Alternative mooring system. Unities: $\mathrm{kNm} / \mathrm{rad}$.
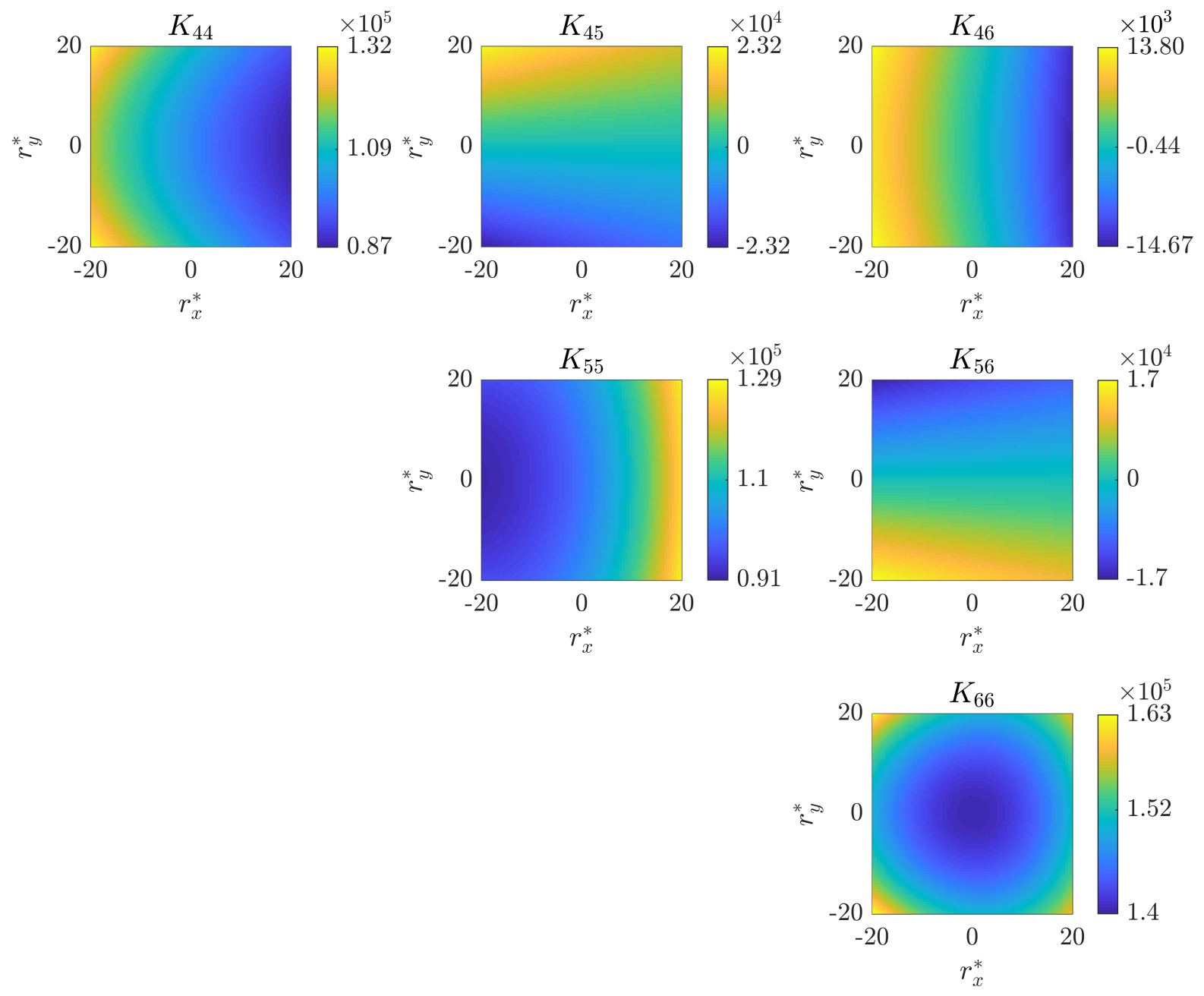

Source: The Author 
Figure 64 - The OC4-DeepCwind natural periods as function of the offset. Alternative mooring system. Unities: s.
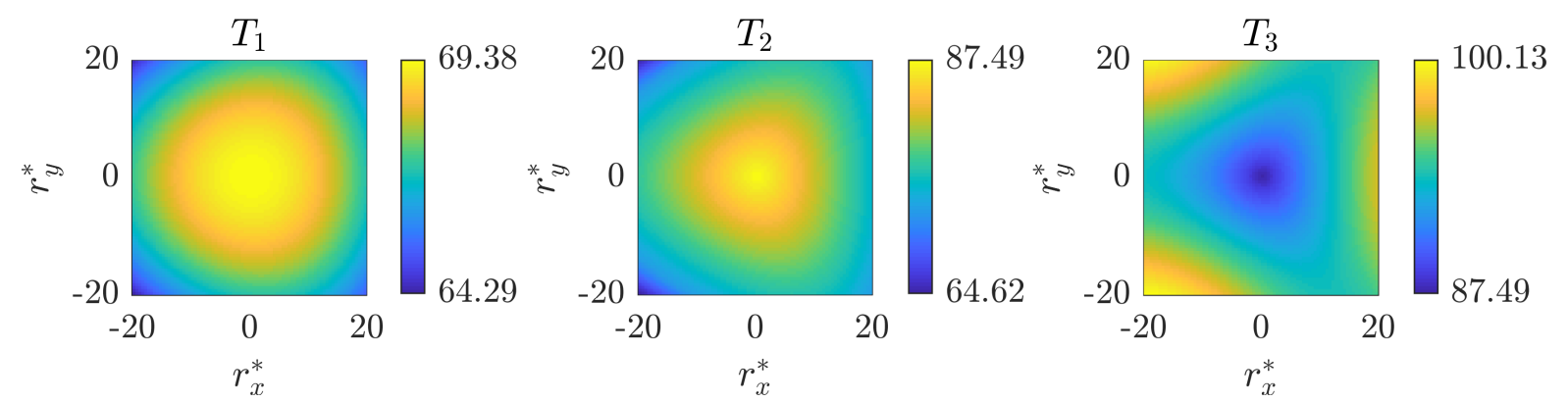

Source: The Author 


\section{Conclusions}

The design of mooring systems is essential for many offshore energy structure projects. A good design regarding its cost effectiveness is even more important for renewable energies. The designer always has to balance the cost - including initial, operational and maintenance ones - and the static and dynamics operational criteria. Additionally, the mooring system design involves multiple variables and their effect on the response is not trivial to be predicted. In this context, analytic expedite tools are of great value provided they can help the designer predict the system response quicker. Consequently, it results in being less expensive, from a computational point-of-view, than the usual high-hierarchical nonlinear time-domain integration scheme. Analytical expedite tools allow to easily change mooring system parameters and analyze their effects on the system stiffness. In this context, the current master dissertation proposed an analytical closed-formulation to assess the mooring system stiffness, regarding the evaluation of effects of the design parameters on a mooring system project.

Initially, the mooring generalized forces were defined. These forces correspond to the restoring ones acting on the vessel and associated with the six generalized coordinates: three translational along $x, y$ and $z$ axes $\left(r_{x}, r_{y}\right.$ and $\left.r_{z}\right)$ and three rotational ones $(\phi, \theta$ and $\psi$ ). Then, a local linearization of these forces was proposed and the stiffness matrix due to the mooring system was defined. The obtained stiffness matrix is completely analytic. The importance of this proposal is to have an initial approximation for the stiffness of the system without complex high-hierarchical numerical modeling, as recommended in many international codes. The methodology was submitted to some case studies, concerning the OC4-DeepCwind semissubmersible platform. Two mooring system arrangements were analyzed: (i) the original OC4 $3 \times 1$ all-chain mooring system and (ii) an alternative $3 \times 1$ all-chain mooring system. The generic and analytical character of the formulation allowed not only to obtain the stiffness coefficients at the trivial equilibrium position, but also to map them as function of the vessel mean offset and heading. The results for the original mooring system at the trivial equilibrium position were compared with the literature and demosntrated excellent accordance. Subsequently natural periods were also assessed, by simply solving the corresponding linear eigenvalue problem for the planar motions (i. e. $r_{x}$, $r_{y}$ and $\psi$ ). Thus, the methodology have been demonstrated as an important and easy tool to evaluate the resonance excitation range. The effects of the pre-tensioning of mooring lines on the system stiffness was evaluated, demonstrating its power for iterative designs.

We conclude that the methodologies herein proposed may be of great value for the design of mooring system projects. The analytic and expedite formulations allow the designer to easily vary the parameters of the mooring system in order to evaluate 
their impact on the response of the whole system. This can help more advanced and sophisticated numerical analyses, contributing for the viability of the project. Additionally, it can also be used as an operation in loco tool, as some operations need as adjustment in the mooring system stiffness in order to desynchronize the vessel natural periods from external forces.

Further work might include reviewing the adopted hypothesis in order to consider the seabed-line interaction and the sea current effect. Keeping the quasi-static modeling, these effects could be considered both in line plane and out-of-plane directions. The eventual 3D final line shape yields fairlead forces defined not only in $h$ and $v$, but also with a component perpendicular to them. Additionally, the analytical model could be used in order to quantify line effects such as the loss of axial stiffness due to creep or even the rupture of one or more lines. Stability analysis, using for example catastrophe sets, and the improvement of the mathematical model considering the line dynamics and hydrodynamics are also possible. 


\section{Bibliography}

AGARWAL, A. K.; JAIN, A. K. Dynamic behavior of offshore spar platforms under regular sea waves. Ocean Engineering, Elsevier BV, v. 30, n. 4, p. 487-516, mar 2003. Cited 2 times at pages 48 and 49 .

AL-SOLIHAT, M. K.; NAHON, M. Stiffness of slack and taut moorings. Ships and Offshore Structures, Informa UK Limited, v. 11, n. 8, p. 890-904, oct 2015. Cited 4 times at pages 50, 54, 55, and 57 .

AMERICAN BUREAU OF STANDARDS (ABS). Rules for building and classing Floating Production Instalations. July, 2014. Cited at page 36.

AMERICAN PETROLEUM INSTITUTE (API). Design and Analysis of Stationkeeping Systems for Floating Structures. October, 2005. Cited at page 35.

BERNITSAS, M. M.; GARZA-RIOS, L. O. Effect of mooring line arrangement on the dynamics of spread mooring systems. Journal of Offshore Mechanics and Arctic Engineering, ASME International, v. 118, n. 1, p. 7, 1996. Cited 6 times at pages 35, 42, $43,44,47$, and 48 .

BERNITSAS, M. M.; KIM, B.-K. Effect of slow-drift loads on nonlinear dynamics of spread mooring systems. Journal of Offshore Mechanics and Arctic Engineering, ASME International, v. 120, n. 4, p. 201, 1998. Cited 2 times at pages 42 and 47.

BROMMUNDT, M. et al. Mooring system optimization for floating wind turbines using frequency domain analysis. Energy Procedia, Elsevier BV, v. 24, p. 289-296, 2012. Cited at page 44 .

BUREAU VERITAS (BV) Classification of mooring systems for permanet and mobile offshore units. December, 2015. Cited 2 times at pages 36 and 51.

CAMPANILE, A.; PISCOPO, V.; SCAMARDELLA, A. Mooring design and selection for floating offshore wind turbines on intermediate and deep water depths. Ocean Engineering, Elsevier BV, v. 148, p. 349-360, jan 2018. Cited 2 times at pages 45 and 46.

DHANAK, M. R.; XIROS, N. I. Springer Handbook of Ocean Engineering. [S.1.]: Springer International Publishing, 2016. Cited 2 times at pages 31 and 32.

DER NORSKE VERITAS (DNV). DNV-OS-E301 Position Mooring. August, 2010. Cited at page 36 . 
DYNEEMA. 2018. Available in: <https://www.dsm.com/products/dyneema/ en_GB/applications/ropes-lines-and-slings/ropes-lines-and-slings-cases/ case-study--mooring-lines-made-with-dyneema-r--still-going-stron.html> .. Date of access 07/02/2020. Cited at page 29 .

FALTINSEN, O. Sea Loads on Ships and Offshore Structures. Cambridge University Press, 1993. (Cambridge Ocean Technology Series). ISBN 9780521458702. Available in: $<$ https://books.google.com.br/books?id=qZq4Rs2DZXoC $>$. Date of access 07/02/2020. Cited 3 times at pages 52, 57, and 74 .

FLORY, J. F.; BANFIELD, S. P.; PETRUSKA, D. J. Defining, measuring, and calculating the properties of fiber rope deepwater mooring lines. In: Offshore Technology Conference. [S.1.]: Offshore Technology Conference, 2004. Cited at page 43.

GARZA-RIOS, L. O.; BERNITSAS, M. M. Effect of size and position of supporting buoys on the dynamics of spread mooring systems. Journal of Offshore Mechanics and Arctic Engineering, ASME International, v. 123, n. 2, p. 49, 2001. Cited 4 times at pages $42,43,47$, and 49 .

GARZA-RIOS, L. O. et al. Dynamics of spread mooring systems with hybrid mooring lines. Journal of Offshore Mechanics and Arctic Engineering, ASME International, v. 122, n. 4, p. 274, 2000. Cited 3 times at pages 42, 47, and 48.

GINCON-SOF. 2018. Available in: < http://www.gicon-sof.de/en/technical-solution.html>. Date of access 07/02/2020. Cited at page 32 .

GUTIÉRREZ-ROMERO, J. E. et al. Non-linear dynamic analysis of the response of moored floating structures. Marine Structures, Elsevier BV, v. 49, p. 116-137, sep 2016. Cited 2 times at pages 54 and 57.

INTERNATIONAL ORGANIZATION FOR STANDARDIZATION (ISO). Petroleum and Natural Gas Industries - Specific Requirements for Offshore Structures, Part \%: Stationkeeping Systems for Floating Offshore Structures and Mobile Offshore Units. 2013. Cited at page 36 .

JONKMAN, J. M. Dynamics modeling and loads analysis of an offshore floating wind turbine. [S.l.], 2007. Cited 2 times at pages 50 and 77.

JOURNÉE, J. M. J.; MASSIE, W. W. Offsore Hydromechanics. [S.l.]: Delft University of Technology, 2001. Cited at page 45.

KASTER, F. et al. DICAS - a new mooring concept for FPSO's. In: Offshore Technology Conference. [S.1.]: Offshore Technology Conference, 1997. Cited at page 30. 
KIM, B. W. et al. Comparison of linear spring and nonlinear FEM methods in dynamic coupled analysis of floating structure and mooring system. Journal of Fluids and Structures, Elsevier BV, v. 42, p. 205-227, oct 2013. Cited 2 times at pages 53 and 57.

KWAN, C.; BRUEN, F. Mooring line dynamics: Comparison of time domain, frequency domain, and quasi-static analyses. In: Offshore Technology Conference. [S.l.]: Offshore Technology Conference, 1991. Cited at page 39.

LOUKOGEORGAKI, E.; ANGELIDES, D. C. Stiffness of mooring lines and performance of floating breakwater in three dimensions. Applied Ocean Research, Elsevier BV, v. 27, n. 4-5, p. 187-208, aug 2005. Cited 5 times at pages 50, 53, 54, 55, and 57.

MA, K.T.; LUO, Y.; KWAN, C.T. T.; W. YONGYAN. Mooring System Engineering for Offshore Structures. System Elsevier Science \& Technology, 2019. ISBN 0128185511. Cited 7 times at pages 35, 36, 37, 38, 39, 40, and 111 .

MACGREGOR. 2017. Available in: < https://www.macgregor.com/Products-solutions/ products/offshore-mooring-systems/pusnes-offshore-mooring-systems/ $>$. Date of access 07/02/2020. Cited at page 29.

MASCIOLA, M.; JONKMAN, J.; ROBERTSON, A. Implementation of a multisegmented, quasi-static cable model. In: INTERNATIONAL SOCIETY OF OFFSHORE AND POLAR ENGINEERS. The Twenty-third International Offshore and Polar Engineering Conference. [S.1.], 2013. Cited 2 times at pages 50 and 51.

MONTASIR, O. A.; YENDURI, A.; KURIAN, V. J. Effect of mooring line configurations on the dynamic responses of truss spar platforms. Ocean Engineering, Elsevier BV, v. 96, p. 161-172, mar 2015. Cited 3 times at pages 45, 54, and 57.

OPPENHEIM, B. W.; WILSON, P. A. Polynomial approximations to mooring forces in equations of low-frequency vessel motions. Journal of Ship Research, 1982. Cited 2 times at pages 47 and 50 .

PESCE, C. P. Technical Publication 7ry - Preliminary Analysis of Moored System Dynamics (in portuguese). [S.1.], 1986. Cited 5 times at pages 41, 47, 52, 53, and 57.

PESCE, C. P.; Amaral, G. A.; Franzini, G. R. Mooring system stiffness: A general analytical formulation with an application to floating offshore wind turbines. In: ASME 2018 1st International Offshore Wind Technical Conference. [S.l.]: ASME, 2018. Cited 10 times at pages 50, 55, 56, 57, 67, 72, 86, 151, 152, and 153 .

ROBERTSON, A. et al. Definition of the Semisubmersible Floating System for Phase II of OC4. [S.1.], 2014. Cited 9 times at pages 32, 54, 57, 79, 80, 82, 83, 84, and 85. 
ROBERTSON, A. et al. Offshore code comparison collaboration continuation within IEA wind task 30: Phase II results regarding a floating semisubmersible wind system. In: Volume 9B: Ocean Renewable Energy. [S.1.]: ASME, 2014. Cited 2 times at pages 86 and 87.

SaemaH. 2019. Available in: $<$ http://www.saemah.com $/$ ?folder $=$ product\&page $=$ mooring $>$. Date of access 07/02/2020. Cited at page 29.

SANNASIRAJ, S. A.; SUNDAR, V.; SUNDARAVADIVELU, R. Mooring forces and motion responses of pontoon-type floating breakwaters. Ocean Engineering, Elsevier BV, v. 25, n. 1, p. 27-48, jan 1998. Cited 3 times at pages 52, 54, and 57.

TRIANTAFYLLOU, M. S. Preliminary desgin of mooring systems. Journal of Ship Research, v. 26, n. 1, p. 25 - 35, mar. 1982. Cited at page 41.

UMAR, A.; DATTA, T. K. Nonlinear response of a moored buoy. Ocean Engineering, v. 30, n. 13 , p. $1625-1646$, set. 2003. Cited at page 49.

WANG, K.; ER, G.-K.; IU, V. P. Nonlinear random vibrations of moored floating structures under seismic and sea wave excitations. Marine Structures, Elsevier BV, v. 65, p. 75-93, may 2019. Cited 2 times at pages 28 and 30.

WEBSTER, W. C. Mooring-induced damping. Ocean Engineering, Elsevier BV, v. 22, n. 6, p. 571-591, aug 1995. Cited at page 40.

WIND POWER OFFSHORE. 2013. Available in: < https://www.windpoweroffshore.com/ article/1210054/foundations-types-depth-limits---alternative-solutions $>$. Date of access 07/02/2020. Cited at page 33 .

YAMAMOTO, T.; YOSHIDA, A.; IJIMA, T. Dynamics of elastically moored floating objects. Applied Ocean Research, Elsevier BV, v. 2, n. 2, p. 85-92, apr 1980. Cited 4 times at pages $47,52,53$, and 57 . 
Appendix 



\section{APPENDIX A - Numerical evaluation of the mooring lines forces and stiffness}

The present Appendix brings a numerical iterative procedure based on the NewtonRaphson algorithm for determining horizontal and vertical forces, as well as the associated stiffness for a multi-segment mooring line in a given configuration, i.e., for a given fairlead position $\left(h_{f}, v_{f}\right)$.

First, let us rewrite the characteristic tension functions as a system of nonlinear equations:

$$
\left\{\begin{array}{l}
e_{1}\left(F_{H}, F_{V}\right) \\
e_{2}\left(F_{H}, F_{V}\right)
\end{array}\right\}=\sum_{j=1}^{N}\left\{\begin{array}{c}
h_{j}\left(F_{H}, F_{V}\right) \\
v_{j}\left(F_{H}, F_{V}\right)
\end{array}\right\}-\left\{\begin{array}{c}
h_{f} \\
v_{f}
\end{array}\right\}
$$

This procedure aims to find the roots $\left(F_{H}^{*}, F_{V}^{*}\right)$ of the nonlinear equation system. In this scenario, the Jacobian matrix of the system is:

$$
\mathbb{J}=\sum_{j=1}^{N} \mathbb{J}_{j}=\sum_{j=1}^{N}\left[\begin{array}{cc}
\frac{\partial h_{j}}{\partial F_{H}} & \frac{\partial h_{j}}{\partial F_{V}} \\
\frac{\partial v_{j}}{\partial F_{H}} & \frac{\partial v_{j}}{\partial F_{V}}
\end{array}\right]
$$

with $^{1}$ :

$$
\begin{aligned}
\frac{\partial h_{1}}{\partial F_{H}} & =\frac{l_{1}}{E A_{1}}-\frac{F_{V 1}}{\gamma_{1}} \frac{1}{\sqrt{F_{H}^{2}+F_{V 1}^{2}}}+\frac{1}{\gamma_{1}} \ln \left(\frac{F_{V 1}+\sqrt{F_{H}^{2}+F_{V 1}^{2}}}{F_{H}}\right) \\
\frac{\partial h_{1}}{\partial F_{V}} & =-\frac{1}{\gamma_{1}} \frac{F_{H}}{\sqrt{F_{H}^{2}+F_{V 1}^{2}}}=\frac{\partial v_{1}}{\partial F_{H}} \\
\frac{\partial v_{1}}{\partial F_{V}} & =\frac{F_{V 1}}{E A_{1} \gamma_{1}}+\frac{F_{V 1}}{\gamma_{1} \sqrt{F_{H}^{2}+F_{V 1}^{2}}}
\end{aligned}
$$

and

$$
\begin{aligned}
\frac{\partial h_{j}}{\partial F_{H}}=\frac{l_{j}}{E A_{j}}-\frac{F_{V j}}{\gamma_{j}} \frac{1}{\sqrt{F_{H}^{2}+F_{V^{2}}^{2}}+\frac{F_{V j-1}}{\gamma_{j}} \frac{1}{\sqrt{F_{H}^{2}+F_{V_{j-1}^{2}}^{2}}}+\frac{1}{\gamma_{j}}} \\
{\left[\ln \left(\frac{F_{V j}+\sqrt{F_{H}^{2}+F_{V_{j}^{2}}^{2}}}{F_{H}}\right)-\ln \left(\frac{F_{V j-1}+\sqrt{F_{H}^{2}+F_{V j-1}^{2}}}{F_{H}}\right)\right] } \\
\frac{\partial h_{j}}{\partial F_{V}}=\frac{F_{H}}{\gamma_{1}}\left(\frac{1}{\sqrt{F_{H}^{2}+F_{V j}^{2}}}-\frac{1}{\sqrt{F_{H}^{2}+F_{V j-1}^{2}}}\right)=\frac{\partial v_{j}}{\partial F_{H}}
\end{aligned}
$$

1 For the case where there is no portion of the first segment laying at the seabed, one should consider Equations A.6 to A.8, for $j=1$. 
$\frac{\partial v_{j}}{\partial F_{V}}=\frac{l_{j}}{E A_{j}}+\frac{1}{\gamma_{1}}\left(\frac{F_{V j}}{\sqrt{F_{H}^{2}+F_{V j}^{2}}}-\frac{F_{V j-1}}{\sqrt{F_{H}^{2}+F_{V j-1}^{2}}}\right)$

Then, it is possible to approximate A.1 around the roots $\left(F_{H}^{*}, F_{V}^{*}\right)$, such as:

$\left\{\begin{array}{l}e_{1}\left(F_{H}, F_{V}\right) \\ e_{2}\left(F_{H}, F_{V}\right)\end{array}\right\} \approx \sum_{j=1}^{N} \mathbb{J}_{j}\left\{\begin{array}{c}F_{H}^{*} \\ F_{V}^{*}\end{array}\right\}$

Or, in matrix form:

$\{E\} \approx \sum_{j=1}^{N} \mathbb{J}_{j}\left\{F^{*}\right\}$

Then, an iterative procedure is proposed based on the Newton-Raphson's method:

$\left\{F^{(k+1)}\right\}=\left\{F^{(k)}\right\}-\mathbb{J}^{(k)^{-1}}\left\{E^{(k)}\right\}$

When the answer is achieved, i.e. when $\left|\left\{F^{(k)}\right\}-\left\{F^{(k+1)}\right\}\right|<\epsilon$, the forces at the fairlead are obtained. The local tangential stiffness coefficients (i.e. $k_{H H}, k_{V V}, k_{H V}$ and $\left.k_{V H}\right)$ are obtained as follow:

$\left[\begin{array}{ll}k_{H H} & k_{H V} \\ k_{V H} & k_{V V}\end{array}\right]=\left[\begin{array}{cc}\frac{\partial F_{H}}{\partial h} & \frac{\partial F_{H}}{\partial v} \\ \frac{\partial F_{V}}{\partial h} & \frac{\partial F_{V}}{\partial v}\end{array}\right]=\mathbb{J}^{(k)^{-1}}$

Notice that as the Jacobian is symmetric, the stiffness matrix also is. 


\section{APPENDIX B - Multi-segment mooring line static configuration}

The present Appendix aims to present a closed formulation to determine the static configuration of a multi-segment mooring line. First of all, it is necessary to determine the mooring line forces, following the methodology proposed in Appendix A. With these forces at hand, it is mandatory to investigate the existence of a portion of the first segment laying at the seabed, i.e. if the vertical component of the mooring force at seabed is null. The following presents a way to confirm or not its existence.

Let us suppose that the whole first segment is suspended. In this case, the vertical component of the mooring line force at the anchor $F_{V_{0}}$ (since there is no TDP) is not null and can be calculated, from Equation 3.98 as:

$$
F_{V_{0}}=F_{V}-\sum_{k=1}^{N} l_{k} \gamma_{k}
$$

Hence, the minimum length that makes the whole first segment be suspended is:

$$
l_{1 \text { min }}=\frac{1}{\gamma_{1}}\left(F_{V}-\sum_{k=2}^{N} l_{k} \gamma_{k}\right)=\frac{F_{V 1}}{\gamma_{1}}
$$

Then, for the case where there is a TDP (i.e. $l_{1}>l_{1 \text { min }}$ ), the suspended length is the minimum length $l_{1 \min }$ itself.

Now, focus is placed on the definition of the static configuration of the multisegment mooring line. The line configuration can be written as function of the curvilinear coordinate $s$, presented in Figure 65. It is possible to define three different positions: (i) $s<l_{r_{1}}$, (ii) $l_{r_{1}}<s<l_{1}$ and (iii) $\sum_{j=1}^{k-1}<s<\sum_{j=1}^{k}$. It follows:

(i) $s<l_{r_{1}}$ ( $s$ belongs to the first segment laying at the seabed portion):

$$
\begin{aligned}
& h(s)=s\left(1+\frac{F_{H}}{E A_{1}}\right) \\
& v(s)=0
\end{aligned}
$$

(ii) $l_{r_{1}}<s<l_{1}$ ( $s$ belongs to the suspended portion of the first segment):

$$
\begin{aligned}
& \left.h(s)=l_{(} r_{1}\right)+\frac{F_{H}}{\gamma_{1}} \ln \left(\frac{F_{V}(s)+\sqrt{F_{H}^{2}+F_{V}^{2}(s)}}{F_{H}}\right)+\frac{F_{H}}{E A_{1}} s \\
& v(s)=\frac{1}{\gamma_{1}}\left(\sqrt{F_{H}^{2}+F_{V}^{2}(s)}-F_{H}\right)+\frac{F_{V}^{2}(s)}{2 \gamma_{1} E A_{1}}
\end{aligned}
$$


Figure 65 - Multi-segment mooring line profile.

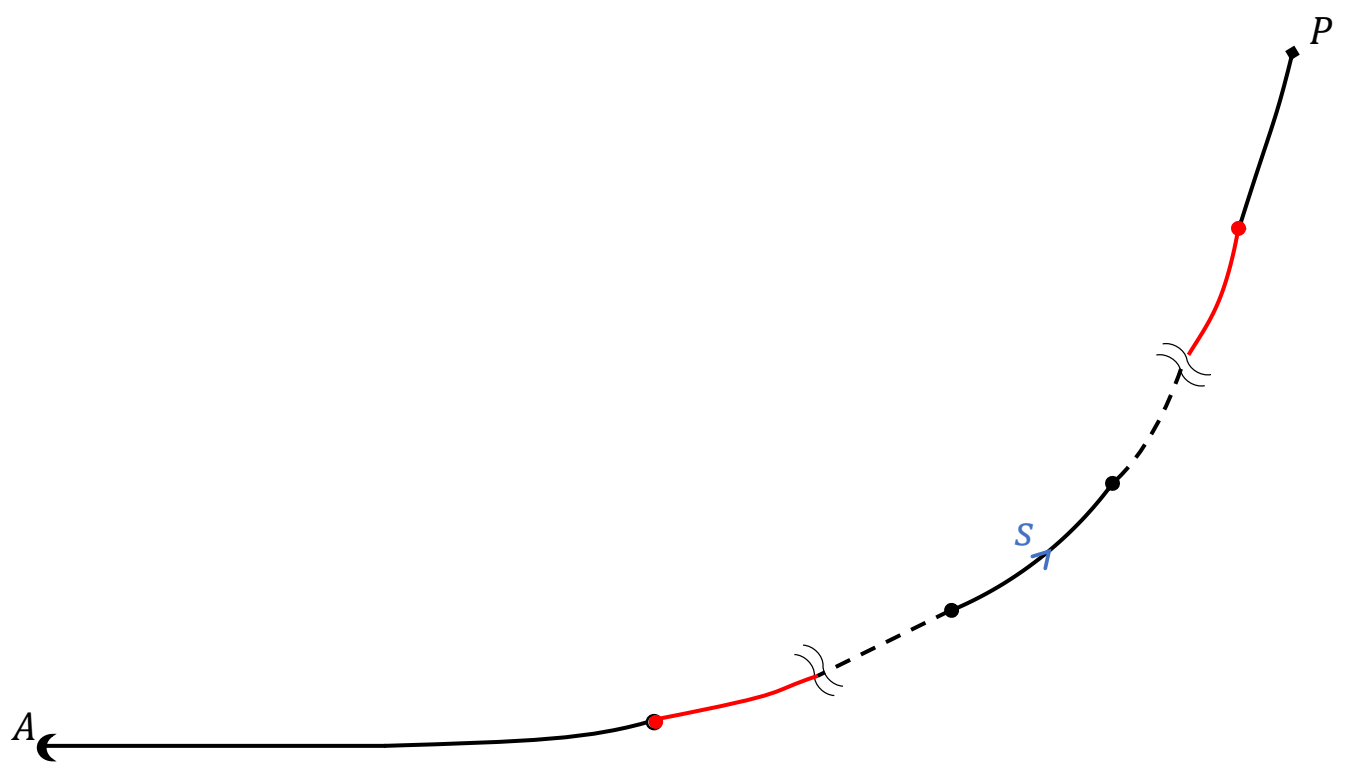

Source: The Author

where the vertical component of the tension at the curvilinear coordinate $s$ is:

$$
F_{V}(s)=\gamma_{1}\left[s-l_{r_{1}}\right]
$$

(iii) $\sum_{j=1}^{k-1}<s<\sum_{j=1}^{k}(s$ belongs to segment $j)$ :

$$
\begin{aligned}
& h(s)=\sum_{j=1}^{k-1} h_{j}+\frac{F_{H}}{\gamma_{1}}\left[\ln \left(\frac{F_{V}^{2}(s)+\sqrt{F_{H}^{2}+F_{V}^{2}(s)}}{F_{H}}\right)-\ln \left(\frac{F_{V_{k-1}}+\sqrt{F_{H}^{2}+F_{V_{k-1}}^{2}}}{F_{H}}\right)\right] \\
& +\frac{F_{H}}{E A_{k}}\left(s-\sum_{j=1}^{k-1} l_{j}\right) \\
& v(s)=\sum_{j=1}^{k-1} v_{j}+\frac{1}{\gamma_{k}}\left(\sqrt{F_{H}^{2}+F_{V}^{2}(s)}-\sqrt{F_{H}^{2}+F_{V_{k-1}}^{2}}\right)+\frac{F_{V}(s)}{E A_{k}}\left(s-\sum_{j=1}^{k-1} l_{j}\right) \\
& -\frac{\gamma_{k}}{E A_{k}}\left(s-\sum_{j=1}^{k-1} l_{j}\right)^{2}
\end{aligned}
$$

where:

$$
F_{V}(s)=F_{V_{j-1}}+\gamma_{j}\left[s-\sum_{j=1}^{j-1} l_{j}\right]
$$

Finally, the angle between the vector tangent to the section and the horizontal $(\theta)$ at coordinate $s$ is given by the relation:

$$
\theta(s)=\arctan \left(\frac{F_{V}(s)}{F_{H}}\right)
$$




\section{APPENDIX C - The local mooring line generalized directions and their projections onto horizontal and vertical planes}

This appendix presents the necessary developments of the local generalized directions $\partial \vec{P}^{(i)} / \partial q_{j}$ and the inner products $\hat{e}_{h}^{(i)} \cdot \partial \vec{P}^{(i)} / \partial q_{j}$ and $\hat{e}_{v}^{(i)} \cdot \partial \vec{P}^{(i)} / \partial q_{j}$ to define the generalized restoring forces from Section 3.2.

- The local generalized directions, $\partial\left\{P^{(i)}\right\}_{E_{x}} / \partial q_{j}$ :

$\frac{\partial \vec{P}^{(i)}}{\partial r_{x}}=\hat{e}_{x}$

$\frac{\partial \vec{P}^{(i)}}{\partial r_{y}}=\hat{e}_{y}$

$\frac{\partial \vec{P}^{(i)}}{\partial r_{z}}=\hat{e}_{z}$

$\frac{\partial \vec{P}^{(i)}}{\partial \phi}=\frac{\partial[\mathbb{R}]_{E_{x} \mid E_{\xi}}}{\partial \phi}\left\{\vec{P}^{(i)}\right\}_{E_{\xi}}$

$\frac{\partial \vec{P}^{(i)}}{\partial \theta}=\frac{\partial[\mathbb{R}]_{E_{x} \mid E_{\xi}}}{\partial \theta}\left\{\vec{P}^{(i)}\right\}_{E_{\xi}}$

$\frac{\partial \vec{P}^{(i)}}{\partial \psi}=\frac{\partial[\mathbb{R}]_{E_{x} \mid E_{\xi}}}{\partial \psi}\left\{\vec{P}^{(i)}\right\}_{E_{\xi}}$

- The inner products $\hat{e}_{h}^{(i)} \cdot \partial \vec{P}^{(i)} / \partial q_{j}$ :

$\hat{e}_{h}^{(i)} \cdot \frac{\partial \vec{P}^{(i)}}{\partial r_{x}}=\cos \alpha^{(i)}$

$\hat{e}_{h}^{(i)} \cdot \frac{\partial \vec{P}^{(i)}}{\partial r_{y}}=\sin \alpha^{(i)}$ 
$\hat{e}_{h}^{(i)} \cdot \frac{\partial \vec{P}^{(i)}}{\partial r_{z}}=0$

$\hat{e}_{h}^{(i)} \cdot \frac{\partial \vec{P}^{(i)}}{\partial \phi}=\cos \alpha^{(i)} \frac{\partial p_{x}^{(i)}}{\partial \phi}+\sin \alpha^{(i)} \frac{\partial p_{y}^{(i)}}{\partial \phi}$

$\hat{e}_{h}^{(i)} \cdot \frac{\partial \vec{P}^{(i)}}{\partial \theta}=\cos \alpha^{(i)} \frac{\partial p_{x}^{(i)}}{\partial \theta}+\sin \alpha^{(i)} \frac{\partial p_{y}^{(i)}}{\partial \theta}$

$\hat{e}_{h}^{(i)} \cdot \frac{\partial \vec{P}^{(i)}}{\partial \psi}=\cos \alpha^{(i)} \frac{\partial p_{x}^{(i)}}{\partial \psi}+\sin \alpha^{(i)} \frac{\partial p_{y}^{(i)}}{\partial \psi}$

- The inner products $\hat{e}_{v}^{(i)} \cdot \partial \vec{P}^{(i)} / \partial q_{j}$ becomes:

$\hat{e}_{v}^{(i)} \cdot \frac{\partial \vec{P}^{(i)}}{\partial r_{x}}=0$

$\hat{e}_{v}^{(i)} \cdot \frac{\partial \vec{P}^{(i)}}{\partial r_{y}}=0$

$\hat{e}_{v}^{(i)} \cdot \frac{\partial \vec{P}^{(i)}}{\partial r_{z}}=-1$

$\hat{e}_{v}^{(i)} \cdot \frac{\partial \vec{P}^{(i)}}{\partial \phi}=-\frac{\partial p_{z}^{(i)}}{\partial \phi}$

$\hat{e}_{v}^{(i)} \cdot \frac{\partial \vec{P}^{(i)}}{\partial \theta}=-\frac{\partial p_{z}^{(i)}}{\partial \theta}$

$\hat{e}_{v}^{(i)} \cdot \frac{\partial \vec{P}^{(i)}}{\partial \psi}=0$ 


\section{APPENDIX D - Intermediate developments for the mooring system stiffness coefficients}

This appendix brings the intermediate developments needed to define the mooring system stiffness matrix: partial derivatives of the distances $h^{(i)}$ and $v^{(i)}$ as well as the inner products $\hat{e}_{h}^{(i)} \cdot \partial \vec{P}^{(i)} / \partial q_{j}$ and $\hat{e}_{v}^{(i)} \cdot \partial \vec{P}^{(i)} / \partial q_{j}$ with respect to $\mathbf{q}$.

- The partial derivatives of $h^{(i)}$ with respect to $q_{j}$ :

$\frac{\partial h^{(i)}}{\partial r_{x}}=-\cos \alpha^{(i)}$

$\frac{\partial h^{(i)}}{\partial r_{y}}=-\sin \alpha^{(i)}$

$\frac{\partial h^{(i)}}{\partial r_{z}}=0$

$\frac{\partial h^{(i)}}{\partial \phi}=-\cos \alpha^{(i)} \frac{\partial p_{x}^{(i)}}{\partial \phi}-\sin \alpha^{(i)} \frac{\partial p_{y}^{(i)}}{\partial \phi}$

$\frac{\partial h^{(i)}}{\partial \theta}=-\cos \alpha^{(i)} \frac{\partial p_{x}^{(i)}}{\partial \theta}-\sin \alpha^{(i)} \frac{\partial p_{y}^{(i)}}{\partial \theta}$

$\frac{\partial h^{(i)}}{\partial \psi}=-\cos \alpha^{(i)} \frac{\partial p_{x}^{(i)}}{\partial \psi}-\sin \alpha^{(i)} \frac{\partial p_{y}^{(i)}}{\partial \psi}$

- The partial derivatives of $v^{(i)}$ with respect to $q_{j}$ :

$\frac{\partial v^{(i)}}{\partial r_{x}}=0$

$\frac{\partial v^{(i)}}{\partial r_{y}}=0$

$\frac{\partial v^{(i)}}{\partial r_{z}}=1$ 
$\frac{\partial v^{(i)}}{\partial \phi}=\frac{\partial p_{z}^{(i)}}{\partial \phi}$

$\frac{\partial v^{(i)}}{\partial \theta}=\frac{\partial p_{z}^{(i)}}{\partial \theta}$

$\frac{\partial v^{(i)}}{\partial \psi}=\frac{\partial p_{z}^{(i)}}{\partial \psi}=0$

- Partial derivatives of the inner products $\hat{e}_{h}^{(i)} \cdot \frac{\partial \vec{P}^{(i)}}{\partial q_{j}}$ :

○ Partial derivatives of $\hat{e}_{h}^{(i)} \cdot \frac{\partial \vec{P}^{(i)}}{\partial r_{x}}$ :

$\frac{\partial}{\partial r_{x}}\left(\hat{e}_{h}^{(i)} \cdot \frac{\partial \vec{P}^{(i)}}{\partial r_{x}}\right)=\frac{1}{h^{(i)}} \sin ^{2} \alpha^{(i)}$

$\frac{\partial}{\partial r_{y}}\left(\hat{e}_{h}^{(i)} \cdot \frac{\partial \vec{P}^{(i)}}{\partial r_{x}}\right)=\frac{1}{h^{(i)}}\left(\cos \alpha^{(i)} \sin \alpha^{(i)}\right)$

$\frac{\partial}{\partial r_{z}}\left(\hat{e}_{h}^{(i)} \cdot \frac{\partial \vec{P}^{(i)}}{\partial r_{x}}\right)=0$

$\frac{\partial}{\partial \phi}\left(\hat{e}_{h}^{(i)} \cdot \frac{\partial \vec{P}^{(i)}}{\partial r_{x}}\right)=\frac{1}{h^{(i)}}\left(-\frac{\partial p_{x}^{(i)}}{\partial \phi} \sin ^{2} \alpha^{(i)}+\frac{\partial p_{y}^{(i)}}{\partial \phi} \sin \alpha^{(i)} \cos \alpha^{(i)}\right)$

$\frac{\partial}{\partial \theta}\left(\hat{e}_{h}^{(i)} \cdot \frac{\partial \vec{P}^{(i)}}{\partial r_{x}}\right)=\frac{1}{h^{(i)}}\left(-\frac{\partial p_{x}^{(i)}}{\partial \theta} \sin ^{2} \alpha^{(i)}+\frac{\partial p_{y}^{(i)}}{\partial \theta} \sin \alpha^{(i)} \cos \alpha^{(i)}\right)$

$\frac{\partial}{\partial \psi}\left(\hat{e}_{h}^{(i)} \cdot \frac{\partial \vec{P}^{(i)}}{\partial r_{x}}\right)=\frac{1}{h^{(i)}}\left(-\frac{\partial p_{x}^{(i)}}{\partial \psi} \sin ^{2} \alpha^{(i)}+\frac{\partial p_{y}^{(i)}}{\partial \psi} \sin \alpha^{(i)} \cos \alpha^{(i)}\right)$

○ Partial derivatives of $\hat{e}_{h}^{(i)} \cdot \frac{\partial \vec{P}^{(i)}}{\partial r_{y}}$ :

$$
\begin{aligned}
& \frac{\partial}{\partial r_{x}}\left(\hat{e}_{h}^{(i)} \cdot \frac{\partial \vec{P}^{(i)}}{\partial r_{y}}\right)=\frac{1}{h^{(i)}} \sin \alpha^{(i)} \cos \alpha^{(i)} \\
& \frac{\partial}{\partial r_{y}}\left(\hat{e}_{h}^{(i)} \cdot \frac{\partial \vec{P}^{(i)}}{\partial r_{y}}\right)=-\frac{1}{h^{(i)}} \cos ^{2} \alpha^{(i)}
\end{aligned}
$$




$$
\begin{aligned}
& \frac{\partial}{\partial r_{z}}\left(\hat{e}_{h}^{(i)} \cdot \frac{\partial \vec{P}^{(i)}}{\partial r_{y}}\right)=0 \\
& \frac{\partial}{\partial \phi}\left(\hat{e}_{h}^{(i)} \cdot \frac{\partial \vec{P}^{(i)}}{\partial r_{y}}\right)=\frac{1}{h^{(i)}}\left(\frac{\partial p_{x}^{(i)}}{\partial \phi} \sin \alpha^{(i)} \cos \alpha^{(i)}-\frac{\partial p_{y}^{(i)}}{\partial \phi} \cos ^{2} \alpha^{(i)}\right) \\
& \frac{\partial}{\partial \theta}\left(\hat{e}_{h}^{(i)} \cdot \frac{\partial \vec{P}^{(i)}}{\partial r_{y}}\right)=\frac{1}{h^{(i)}}\left(\frac{\partial p_{x}^{(i)}}{\partial \theta} \sin \alpha^{(i)} \cos \alpha^{(i)}-\frac{\partial p_{y}^{(i)}}{\partial \theta} \cos ^{2} \alpha^{(i)}\right) \\
& \frac{\partial}{\partial \psi}\left(\hat{e}_{h}^{(i)} \cdot \frac{\partial \vec{P}^{(i)}}{\partial r_{y}}\right)=\frac{1}{h^{(i)}}\left(\frac{\partial p_{x}^{(i)}}{\partial \psi} \sin \alpha^{(i)} \cos \alpha^{(i)}-\frac{\partial p_{y}^{(i)}}{\partial \psi} \cos ^{2} \alpha^{(i)}\right)
\end{aligned}
$$

○ Partial derivatives of $\hat{e}_{h}^{(i)} \cdot \frac{\partial \vec{P}^{(i)}}{\partial r_{z}}$ :

$$
\begin{aligned}
& \frac{\partial}{\partial r_{x}}\left(\hat{e}_{h}^{(i)} \cdot \frac{\partial \vec{P}^{(i)}}{\partial r_{z}}\right)=0 \\
& \frac{\partial}{\partial r_{y}}\left(\hat{e}_{h}^{(i)} \cdot \frac{\partial \vec{P}^{(i)}}{\partial r_{z}}\right)=0 \\
& \frac{\partial}{\partial r_{z}}\left(\hat{e}_{h}^{(i)} \cdot \frac{\partial \vec{P}^{(i)}}{\partial r_{z}}\right)=0 \\
& \frac{\partial}{\partial r_{\phi}}\left(\hat{e}_{h}^{(i)} \cdot \frac{\partial \vec{P}^{(i)}}{\partial r_{z}}\right)=0 \\
& \frac{\partial}{\partial r_{\theta}}\left(\hat{e}_{h}^{(i)} \cdot \frac{\partial \vec{P}^{(i)}}{\partial r_{z}}\right)=0 \\
& \frac{\partial}{\partial r_{\psi}}\left(\hat{e}_{h}^{(i)} \cdot \frac{\partial \vec{P}^{(i)}}{\partial r_{z}}\right)=0
\end{aligned}
$$

○ Partial derivatives of $\hat{e}_{h}^{(i)} \cdot \frac{\partial \vec{P}^{(i)}}{\partial \phi}$ :

$$
\frac{\partial}{\partial r_{x}}\left(\hat{e}_{h}^{(i)} \cdot \frac{\partial \vec{P}^{(i)}}{\partial \phi}\right)=\frac{1}{h^{(i)}}\left(-\sin ^{2} \alpha^{(i)} \frac{\partial p_{x}^{(i)}}{\partial \phi}+\sin \alpha^{(i)} \cos \alpha^{(i)} \frac{\partial p_{y}^{(i)}}{\partial \phi}\right)
$$




$$
\begin{aligned}
& \frac{\partial}{\partial r_{y}}\left(\hat{e}_{h}^{(i)} \cdot \frac{\partial \vec{P}^{(i)}}{\partial \phi}\right)=\frac{1}{h^{(i)}}\left(\sin \alpha^{(i)} \cos \alpha^{(i)} \frac{\partial p_{x}^{(i)}}{\partial \phi}-\cos ^{2} \alpha^{(i)} \frac{\partial p_{y}^{(i)}}{\partial \phi}\right) \\
& \frac{\partial}{\partial r_{z}}\left(\hat{e}_{h}^{(i)} \cdot \frac{\partial \vec{P}^{(i)}}{\partial \phi}\right)=0 \\
& \frac{\partial}{\partial \phi}\left(\hat{e}_{h}^{(i)} \cdot \frac{\partial \vec{P}^{(i)}}{\partial \phi}\right)=\cos \alpha^{(i)} \frac{\partial^{2} p_{x}^{(i)}}{\partial \phi^{2}}+\sin \alpha^{(i)} \frac{\partial^{2} p_{y}^{(i)}}{\partial \phi^{2}}+ \\
& \frac{1}{h^{(i)}}\left[-\left(\frac{\partial p_{x}^{(i)}}{\partial \phi}\right)^{2} \sin ^{2} \alpha^{(i)}+2 \frac{\partial p_{x}^{(i)}}{\partial \phi} \frac{\partial p_{y}^{(i)}}{\partial \phi} \sin \alpha^{(i)} \cos \alpha^{(i)}-\left(\frac{\partial p_{y}^{(i)}}{\partial \phi}\right)^{2} \cos ^{2} \alpha^{(i)}\right]
\end{aligned}
$$

$$
\begin{gathered}
\frac{\partial}{\partial \theta}\left(\hat{e}_{h}^{(i)} \cdot \frac{\partial \vec{P}^{(i)}}{\partial \phi}\right)=\cos \alpha^{(i)} \frac{\partial^{2} p_{x}^{(i)}}{\partial \phi \partial \theta}+\sin \alpha^{(i)} \frac{\partial^{2} p_{y}^{(i)}}{\partial \phi \partial \theta}+ \\
\frac{1}{h^{(i)}}\left(-\frac{\partial p_{x}^{(i)}}{\partial \phi} \frac{\partial p_{x}^{(i)}}{\partial \theta} \sin ^{2} \alpha^{(i)}+\frac{\partial p_{x}^{(i)}}{\partial \phi} \frac{\partial p_{y}^{(i)}}{\partial \theta} \sin \alpha^{(i)} \cos \alpha^{(i)}\right)+ \\
\frac{1}{h^{(i)}}\left(\frac{\partial p_{x}^{(i)}}{\partial \theta} \frac{\partial p_{y}^{(i)}}{\partial \phi} \sin \alpha^{(i)} \cos \alpha^{(i)}-\frac{\partial p_{y}^{(i)}}{\partial \phi} \frac{\partial p_{y}^{(i)}}{\partial \theta} \cos ^{2} \alpha^{(i)}\right)
\end{gathered}
$$

$$
\begin{aligned}
& \frac{\partial}{\partial \psi}\left(\hat{e}_{h}^{(i)} \cdot \frac{\partial \vec{P}^{(i)}}{\partial \phi}\right)= \cos \alpha^{(i)} \frac{\partial^{2} p_{x}^{(i)}}{\partial \phi \partial \psi}+\sin \alpha^{(i)} \frac{\partial^{2} p_{y}^{(i)}}{\partial \phi \partial \psi}+ \\
& \frac{1}{h^{(i)}}\left(-\frac{\partial p_{x}^{(i)}}{\partial \phi} \frac{\partial p_{x}^{(i)}}{\partial \psi} \sin ^{2} \alpha^{(i)}+\frac{\partial p_{x}^{(i)}}{\partial \phi} \frac{\partial p_{y}^{(i)}}{\partial \psi} \sin \alpha^{(i)} \cos \alpha^{(i)}\right)+ \\
& \frac{1}{h^{(i)}}\left(+\frac{\partial p_{x}^{(i)}}{\partial \psi} \frac{\partial p_{y}^{(i)}}{\partial \phi} \sin \alpha^{(i)} \cos \alpha^{(i)}-\frac{\partial p_{y}^{(i)}}{\partial \phi} \frac{\partial p_{y}^{(i)}}{\partial \psi} \cos ^{2} \alpha^{(i)}\right)
\end{aligned}
$$

○ Partial derivatives of $\hat{e}_{h}^{(i)} \cdot \frac{\partial \vec{P}^{(i)}}{\partial \theta}$ :

$$
\begin{aligned}
& \frac{\partial}{\partial r_{x}}\left(\hat{e}_{h}^{(i)} \cdot \frac{\partial \vec{P}^{(i)}}{\partial \theta}\right)=\frac{1}{h^{(i)}}\left(-\sin ^{2} \alpha^{(i)} \frac{\partial p_{x}^{(i)}}{\partial \theta}+\frac{1}{h^{(i)}} \sin \alpha^{(i)} \cos \alpha^{(i)} \frac{\partial p_{y}^{(i)}}{\partial \theta}\right) \\
& \frac{\partial}{\partial r_{y}}\left(\hat{e}_{h}^{(i)} \cdot \frac{\partial \vec{P}^{(i)}}{\partial \theta}\right)=\frac{1}{h^{(i)}}\left(\sin \alpha^{(i)} \cos \alpha^{(i)} \frac{\partial p_{x}^{(i)}}{\partial \theta}-\cos ^{2} \alpha^{(i)} \frac{\partial p_{y}^{(i)}}{\partial \theta}\right) \\
& \frac{\partial}{\partial r_{z}}\left(\hat{e}_{h}^{(i)} \cdot \frac{\partial \vec{P}^{(i)}}{\partial \theta}\right)=0
\end{aligned}
$$




$$
\begin{gathered}
\frac{\partial}{\partial \phi}\left(\hat{e}_{h}^{(i)} \cdot \frac{\partial \vec{P}^{(i)}}{\partial \theta}\right)=\cos \alpha^{(i)} \frac{\partial^{2} p_{x}^{(i)}}{\partial \theta \partial \phi}+\sin \alpha^{(i)} \frac{\partial^{2} p_{y}^{(i)}}{\partial \theta \partial \phi}+ \\
\frac{1}{h^{(i)}}\left(-\frac{\partial p_{x}^{(i)}}{\partial \theta} \frac{\partial p_{x}^{(i)}}{\partial \phi} \sin ^{2} \alpha^{(i)}+\frac{\partial p_{x}^{(i)}}{\partial \theta} \frac{\partial p_{y}^{(i)}}{\partial \phi} \sin \alpha^{(i)} \cos \alpha^{(i)}\right)+ \\
\frac{1}{h^{(i)}}\left(\frac{\partial p_{x}^{(i)}}{\partial \phi} \frac{\partial p_{y}^{(i)}}{\partial \theta} \sin \alpha^{(i)} \cos \alpha^{(i)}-\frac{\partial p_{y}^{(i)}}{\partial \theta} \frac{\partial p_{y}^{(i)}}{\partial \phi} \cos ^{2} \alpha^{(i)}\right)
\end{gathered}
$$

$$
\begin{aligned}
& \frac{\partial}{\partial \theta}\left(\hat{e}_{h}^{(i)} \cdot \frac{\partial \vec{P}^{(i)}}{\partial \theta}\right)=\cos \alpha^{(i)} \frac{\partial^{2} p_{x}^{(i)}}{\partial \theta^{2}}+\sin \alpha^{(i)} \frac{\partial^{2} p_{y}^{(i)}}{\partial \theta^{2}}+ \\
& \frac{1}{h^{(i)}}\left[-\left(\frac{\partial p_{x}^{(i)}}{\partial \theta}\right)^{2} \sin ^{2} \alpha^{(i)}+2 \frac{\partial p_{x}^{(i)}}{\partial \theta} \frac{\partial p_{y}^{(i)}}{\partial \theta} \sin \alpha^{(i)} \cos \alpha^{(i)}-\left(\frac{\partial p_{y}^{(i)}}{\partial \theta}\right)^{2} \cos ^{2} \alpha^{(i)}\right]
\end{aligned}
$$

$$
\begin{aligned}
& \frac{\partial}{\partial \psi}\left(\hat{e}_{h}^{(i)} \cdot \frac{\partial \vec{P}^{(i)}}{\partial \theta}\right)= \cos \alpha^{(i)} \frac{\partial^{2} p_{x}^{(i)}}{\partial \theta \partial \psi}+\sin \alpha^{(i)} \frac{\partial^{2} p_{y}^{(i)}}{\partial \theta \partial \psi}+ \\
& \frac{1}{h^{(i)}}\left(-\frac{\partial p_{x}^{(i)}}{\partial \theta} \frac{\partial p_{x}^{(i)}}{\partial \psi} \sin ^{2} \alpha^{(i)}+\frac{\partial p_{x}^{(i)}}{\partial \theta} \frac{\partial p_{y}^{(i)}}{\partial \psi} \sin \alpha^{(i)} \cos \alpha^{(i)}\right)+ \\
& \frac{1}{h^{(i)}}\left(\frac{\partial p_{x}^{(i)}}{\partial \psi} \frac{\partial p_{y}^{(i)}}{\partial \theta} \sin \alpha^{(i)} \cos \alpha^{(i)}-\frac{\partial p_{y}^{(i)}}{\partial \theta} \frac{\partial p_{y}^{(i)}}{\partial \psi} \cos ^{2} \alpha^{(i)}\right)
\end{aligned}
$$

○ Partial derivatives of $\hat{e}_{h}^{(i)} \cdot \frac{\partial \vec{P}^{(i)}}{\partial \psi}$ :

$$
\begin{aligned}
\frac{\partial}{\partial r_{x}}\left(\hat{e}_{h}^{(i)} \cdot \frac{\partial \vec{P}^{(i)}}{\partial \psi}\right) & =\frac{1}{h^{(i)}}\left(-\sin ^{2} \alpha^{(i)} \frac{\partial p_{x}^{(i)}}{\partial \psi}+\sin \alpha^{(i)} \cos \alpha^{(i)} \frac{\partial p_{y}^{(i)}}{\partial \psi}\right) \\
\frac{\partial}{\partial r_{y}}\left(\hat{e}_{h}^{(i)} \cdot \frac{\partial \vec{P}^{(i)}}{\partial \psi}\right) & =\frac{1}{h^{(i)}}\left(\sin \alpha^{(i)} \cos \alpha^{(i)} \frac{\partial p_{x}^{(i)}}{\partial \psi}+\cos ^{2} \alpha^{(i)} \frac{\partial p_{y}^{(i)}}{\partial \psi}\right) \\
\frac{\partial}{\partial r_{z}}\left(\hat{e}_{h}^{(i)} \cdot \frac{\partial \vec{P}^{(i)}}{\partial \psi}\right) & =0 \\
\frac{\partial}{\partial \phi}\left(\hat{e}_{h}^{(i)} \cdot \frac{\partial \vec{P}^{(i)}}{\partial \psi}\right) & =\cos \alpha^{(i)} \frac{\partial^{2} p_{x}^{(i)}}{\partial \psi \partial \phi}+\sin \alpha^{(i)} \frac{\partial^{2} p_{y}^{(i)}}{\partial \psi \partial \phi}+ \\
\frac{1}{h^{(i)}}\left(-\frac{\partial p_{x}^{(i)}}{\partial \psi} \frac{\partial p_{x}^{(i)}}{\partial \phi} \sin ^{2} \alpha^{(i)}+\frac{\partial p_{x}^{(i)}}{\partial \psi} \frac{\partial p_{y}^{(i)}}{\partial \phi} \sin \alpha^{(i)} \cos \alpha^{(i)}\right)+ & \frac{1}{h^{(i)}}\left(\frac{\partial p_{x}^{(i)}}{\partial \phi} \frac{\partial p_{y}^{(i)}}{\partial \psi} \sin \alpha^{(i)} \cos \alpha^{(i)}-\frac{\partial p_{y}^{(i)}}{\partial \psi} \frac{\partial p_{y}^{(i)}}{\partial \phi} \cos ^{2} \alpha^{(i)}\right)
\end{aligned}
$$




$$
\begin{gathered}
\frac{\partial}{\partial \theta}\left(\hat{e}_{h}^{(i)} \cdot \frac{\partial \vec{P}^{(i)}}{\partial \psi}\right)=\cos \alpha^{(i)} \frac{\partial^{2} p_{x}^{(i)}}{\partial \psi \partial \theta}+\sin \alpha^{(i)} \frac{\partial^{2} p_{y}^{(i)}}{\partial \psi \partial \theta}+ \\
\frac{1}{h^{(i)}}\left(-\frac{\partial p_{x}^{(i)}}{\partial \psi} \frac{\partial p_{x}^{(i)}}{\partial \theta} \sin ^{2} \alpha^{(i)}+\frac{\partial p_{x}^{(i)}}{\partial \psi} \frac{\partial p_{y}^{(i)}}{\partial \theta} \sin \alpha^{(i)} \cos \alpha^{(i)}\right)+ \\
\frac{1}{h^{(i)}}\left(\frac{\partial p_{x}^{(i)}}{\partial \theta} \frac{\partial p_{y}^{(i)}}{\partial \psi} \sin \alpha^{(i)} \cos \alpha^{(i)}-\frac{\partial p_{y}^{(i)}}{\partial \psi} \frac{\partial p_{y}^{(i)}}{\partial \theta} \cos ^{2} \alpha^{(i)}\right)
\end{gathered}
$$

$$
\begin{aligned}
\frac{\partial}{\partial \psi}\left(\hat{e}_{h}^{(i)} \cdot \frac{\partial \vec{P}^{(i)}}{\partial \psi}\right)=\cos \alpha^{(i)} \frac{\partial^{2} p_{x}^{(i)}}{\partial \psi^{2}}+\sin \alpha^{(i)} \frac{\partial^{2} p_{y}^{(i)}}{\partial \psi^{2}}+ \\
\frac{1}{h^{(i)}}\left[-\left(\frac{\partial p_{x}^{(i)}}{\partial \psi}\right)^{2} \sin ^{2} \alpha^{(i)}+2 \frac{\partial p_{x}^{(i)}}{\partial \psi} \frac{\partial p_{y}^{(i)}}{\partial \psi} \sin \alpha^{(i)} \cos \alpha^{(i)}-\left(\frac{\partial p_{y}^{(i)}}{\partial \psi}\right)^{2} \cos ^{2} \alpha^{(i)}\right]
\end{aligned}
$$

- Partial derivatives of the inner products $\hat{e}_{v}^{(i)} \cdot \frac{\partial \vec{P}^{(i)}}{\partial q_{j}}$ :

○ Partial derivatives of $\hat{e}_{v}^{(i)} \cdot \frac{\partial \vec{P}^{(i)}}{\partial r_{x}}$ :

$$
\begin{gathered}
\frac{\partial}{\partial r_{x}}\left(\hat{e}_{v}^{(i)} \cdot \frac{\partial \vec{P}^{(i)}}{\partial r_{x}}\right)=0 \\
\frac{\partial}{\partial r_{y}}\left(\hat{e}_{v}^{(i)} \cdot \frac{\partial \vec{P}^{(i)}}{\partial r_{x}}\right)=0
\end{gathered}
$$

$$
\frac{\partial}{\partial r_{z}}\left(\hat{e}_{v}^{(i)} \cdot \frac{\partial \vec{P}^{(i)}}{\partial r_{x}}\right)=0
$$$$
\frac{\partial}{\partial \phi}\left(\hat{e}_{v}^{(i)} \cdot \frac{\partial \vec{P}^{(i)}}{\partial r_{x}}\right)=0
$$

$\frac{\partial}{\partial \theta}\left(\hat{e}_{v}^{(i)} \cdot \frac{\partial \vec{P}^{(i)}}{\partial r_{x}}\right)=0$

$$
\frac{\partial}{\partial \psi}\left(\hat{e}_{v}^{(i)} \cdot \frac{\partial \vec{P}^{(i)}}{\partial r_{x}}\right)=0
$$

○ Partial derivatives of $\hat{e}_{v}^{(i)} \cdot \frac{\partial \vec{P}^{(i)}}{\partial r_{y}}$ :

$$
\frac{\partial}{\partial r_{x}}\left(\hat{e}_{v}^{(i)} \cdot \frac{\partial \vec{P}^{(i)}}{\partial r_{y}}\right)=0
$$




$$
\begin{aligned}
& \frac{\partial}{\partial r_{y}}\left(\hat{e}_{v}^{(i)} \cdot \frac{\partial \vec{P}^{(i)}}{\partial r_{y}}\right)=0 \\
& \frac{\partial}{\partial r_{z}}\left(\hat{e}_{v}^{(i)} \cdot \frac{\partial \vec{P}^{(i)}}{\partial r_{y}}\right)=0 \\
& \frac{\partial}{\partial \phi}\left(\hat{e}_{v}^{(i)} \cdot \frac{\partial \vec{P}^{(i)}}{\partial r_{y}}\right)=0 \\
& \frac{\partial}{\partial \theta}\left(\hat{e}_{v}^{(i)} \cdot \frac{\partial \vec{P}^{(i)}}{\partial r_{y}}\right)=0 \\
& \frac{\partial}{\partial \psi}\left(\hat{e}_{v}^{(i)} \cdot \frac{\partial \vec{P}^{(i)}}{\partial r_{y}}\right)=0
\end{aligned}
$$

○ Partial derivatives of $\hat{e}_{v}^{(i)} \cdot \frac{\partial \vec{P}^{(i)}}{\partial r_{z}}$ :

$$
\begin{aligned}
& \frac{\partial}{\partial r_{x}}\left(\hat{e}_{v}^{(i)} \cdot \frac{\partial \vec{P}^{(i)}}{\partial r_{z}}\right)=0 \\
& \frac{\partial}{\partial r_{y}}\left(\hat{e}_{v}^{(i)} \cdot \frac{\partial \vec{P}^{(i)}}{\partial r_{z}}\right)=0
\end{aligned}
$$

$$
\frac{\partial}{\partial r_{z}}\left(\hat{e}_{v}^{(i)} \cdot \frac{\partial \vec{P}^{(i)}}{\partial r_{z}}\right)=0
$$

$$
\frac{\partial}{\partial r_{\phi}}\left(\hat{e}_{v}^{(i)} \cdot \frac{\partial \vec{P}^{(i)}}{\partial r_{z}}\right)=0
$$

$$
\frac{\partial}{\partial r_{\theta}}\left(\hat{e}_{v}^{(i)} \cdot \frac{\partial \vec{P}^{(i)}}{\partial r_{z}}\right)=0
$$

$$
\frac{\partial}{\partial r_{\psi}}\left(\hat{e}_{v}^{(i)} \cdot \frac{\partial \vec{P}^{(i)}}{\partial r_{z}}\right)=0
$$


○ Partial derivatives of $\hat{e}_{v}^{(i)} \cdot \frac{\partial \vec{P}^{(i)}}{\partial \phi}$ :

$\frac{\partial}{\partial r_{x}}\left(\hat{e}_{v}^{(i)} \cdot \frac{\partial \vec{P}^{(i)}}{\partial \phi}\right)=\frac{\partial}{\partial r_{x}}\left(\frac{\partial p_{z}^{(i)}}{\partial \phi}\right)=0$

$\frac{\partial}{\partial r_{y}}\left(\hat{e}_{v}^{(i)} \cdot \frac{\partial \vec{P}^{(i)}}{\partial \phi}\right)=\frac{\partial}{\partial r_{y}}\left(\frac{\partial p_{z}^{(i)}}{\partial \phi}\right)=0$

$\frac{\partial}{\partial r_{z}}\left(\hat{e}_{v}^{(i)} \cdot \frac{\partial \vec{P}^{(i)}}{\partial \phi}\right)=0$

$\frac{\partial}{\partial \phi}\left(\hat{e}_{v}^{(i)} \cdot \frac{\partial \vec{P}^{(i)}}{\partial \phi}\right)=-\frac{\partial^{2} p_{z}^{(i)}}{\partial \phi^{2}}$

$\frac{\partial}{\partial \theta}\left(\hat{e}_{v}^{(i)} \cdot \frac{\partial \vec{P}^{(i)}}{\partial \phi}\right)=-\frac{\partial^{2} p_{z}^{(i)}}{\partial \phi \partial \theta}$

$\frac{\partial}{\partial \psi}\left(\hat{e}_{v}^{(i)} \cdot \frac{\partial \vec{P}^{(i)}}{\partial \phi}\right)=0$

○ Partial derivatives of $\hat{e}_{v}^{(i)} \cdot \frac{\partial \vec{P}^{(i)}}{\partial \theta}$

$\frac{\partial}{\partial r_{x}}\left(\hat{e}_{v}^{(i)} \cdot \frac{\partial \vec{P}^{(i)}}{\partial \theta}\right)=0$

$\frac{\partial}{\partial r_{y}}\left(\hat{e}_{v}^{(i)} \cdot \frac{\partial \vec{P}^{(i)}}{\partial \theta}\right)=0$

$\frac{\partial}{\partial r_{z}}\left(\hat{e}_{v}^{(i)} \cdot \frac{\partial \vec{P}^{(i)}}{\partial \theta}\right)=0$

$\frac{\partial}{\partial \phi}\left(\hat{e}_{v}^{(i)} \cdot \frac{\partial \vec{P}^{(i)}}{\partial \theta}\right)=-\frac{\partial^{2} p_{z}^{(i)}}{\partial \theta \partial \phi}$

$\frac{\partial}{\partial \theta}\left(\hat{e}_{v}^{(i)} \cdot \frac{\partial \vec{P}^{(i)}}{\partial \theta}\right)=-\frac{\partial^{2} p_{z}^{(i)}}{\partial \theta^{2}}$ 
$\frac{\partial}{\partial \psi}\left(\hat{e}_{v}^{(i)} \cdot \frac{\partial \vec{P}^{(i)}}{\partial \theta}\right)=0$

○ Partial derivatives of $\hat{e}_{v}^{(i)} \cdot \frac{\partial \vec{P}(i)}{\partial \psi}$

$$
\begin{aligned}
& \frac{\partial}{\partial r_{x}}\left(\hat{e}_{v}^{(i)} \cdot \frac{\partial \vec{P}^{(i)}}{\partial \psi}\right)=0 \\
& \frac{\partial}{\partial r_{y}}\left(\hat{e}_{v}^{(i)} \cdot \frac{\partial \vec{P}^{(i)}}{\partial \psi}\right)=0 \\
& \frac{\partial}{\partial r_{z}}\left(\hat{e}_{v}^{(i)} \cdot \frac{\partial \vec{P}(i)}{\partial \psi}\right)=0 \\
& \frac{\partial}{\partial \phi}\left(\hat{e}_{v}^{(i)} \cdot \frac{\partial \vec{P}^{(i)}}{\partial \psi}\right)=0 \\
& \frac{\partial}{\partial \theta}\left(\hat{e}_{v}^{(i)} \cdot \frac{\partial \vec{P}^{(i)}}{\partial \psi}\right)=0 \\
& \frac{\partial}{\partial \psi}\left(\hat{e}_{v}^{(i)} \cdot \frac{\partial \vec{P}^{(i)}}{\partial \psi}\right)=0
\end{aligned}
$$





\section{APPENDIX E - Algebraic manipulation of the stiffness coefficients}

- First line coefficients - coefficients associated with a displacement $r_{x}$ :

$$
\begin{aligned}
& K_{14}^{(i)}= \frac{\partial p_{x}^{(i)}}{\partial \phi}\left(k_{H H}^{(i)} \cos ^{2} \alpha^{(i)}+\bar{k}_{H H}^{(i)} \sin ^{2} \alpha^{(i)}\right)+ \\
& \frac{\partial p_{y}^{(i)}}{\partial \phi}\left(k_{H H}^{(i)}-\bar{k}_{H H}^{(i)}\right) \sin \alpha^{(i)} \cos \alpha^{(i)}-\frac{\partial p_{z}^{(i)}}{\partial \phi} k_{H V}^{(i)} \cos \alpha^{(i)} \\
&= \frac{\partial p_{x}^{(i)}}{\partial \phi} K_{11}^{(i)}+\frac{\partial p_{y}^{(i)}}{\partial \phi} K_{12}^{(i)}+\frac{\partial p_{z}^{(i)}}{\partial \phi} K_{13}^{(i)} \\
& K_{15}^{(i)}= \frac{\partial p_{x}^{(i)}}{\partial \theta}\left(k_{H H}^{(i)} \cos ^{2} \alpha^{(i)}+\bar{k}_{H H}^{(i)} \sin ^{2} \alpha^{(i)}\right)+ \\
& \frac{\partial p_{y}^{(i)}}{\partial \theta}\left(k_{H H}^{(i)}-\bar{k}_{H H}^{(i)}\right) \sin \alpha^{(i)} \cos \alpha^{(i)}-\frac{\partial p_{z}^{(i)}}{\partial \theta} k_{H V} \cos \alpha^{(i)} \frac{\partial p_{x}^{(i)}}{\partial \theta} K_{11}^{(i)}+\frac{\partial p_{y}^{(i)}}{\partial \theta} K_{12}^{(i)}+\frac{\partial p_{z}^{(i)}}{\partial \theta} K_{13}^{(i)} \\
& K_{16}^{(i)}= \frac{\partial p_{x}^{(i)}}{\partial \psi}\left(k_{H H}^{(i)} \cos ^{2} \alpha^{(i)}+\bar{k}_{H H}^{(i)} \sin ^{2} \alpha^{(i)}\right)+ \\
& \frac{\partial p_{y}^{(i)}}{\partial \psi}\left(k_{H H}^{(i)}-\bar{k}_{H H}^{(i)}\right) \sin \alpha^{(i)} \cos \alpha^{(i)}=\frac{\partial p_{x}^{(i)}}{\partial \psi} K_{11}^{(i)}+\frac{\partial p_{y}^{(i)}}{\partial \psi} K_{12}^{(i)}
\end{aligned}
$$

- Second line coefficients - coefficients associated with a displacement $r_{y}$ :

$$
\begin{aligned}
& K_{24}^{(i)}= \frac{\partial p_{x}^{(i)}}{\partial \phi}\left(k_{H H}^{(i)}-\bar{k}_{H H}^{(i)}\right) \sin \alpha^{(i)} \cos \alpha^{(i)}+ \\
& \frac{\partial p_{y}^{(i)}}{\partial \phi}\left(k_{H H}^{(i)} \cos ^{2} \alpha^{(i)}+\bar{k}_{H H}^{(i)} \sin ^{2} \alpha^{(i)}\right)-\frac{\partial p_{z}^{(i)}}{\partial \phi} k_{H V} \sin \alpha^{(i)} \\
&=\frac{\partial p_{x}^{(i)}}{\partial \phi} K_{21}^{(i)}+\frac{\partial p_{y}^{(i)}}{\partial \phi} K_{22}^{(i)}+\frac{\partial p_{z}^{(i)}}{\partial \phi} K_{23}^{(i)} \\
& K_{25}^{(i)}=\frac{\partial p_{x}^{(i)}}{\partial \theta}\left(k_{H H}^{(i)}-\bar{k}_{H H}^{(i)}\right) \sin \alpha^{(i)} \cos \alpha^{(i)}+ \\
& \frac{\partial p_{y}^{(i)}}{\partial \theta}\left(k_{H H}^{(i)} \cos ^{2} \alpha^{(i)}+\bar{k}_{H H}^{(i)} \sin ^{2} \alpha^{(i)}\right)-\frac{\partial p_{z}^{(i)}}{\partial \theta} k_{H V} \sin \alpha^{(i)} \\
&=\frac{\partial p_{x}^{(i)}}{\partial \theta} K_{21}^{(i)}+\frac{\partial p_{y}^{(i)}}{\partial \theta} K_{22}^{(i)}+\frac{\partial p_{z}^{(i)}}{\partial \theta} K_{23}^{(i)}
\end{aligned}
$$




$$
\begin{aligned}
K_{26}^{(i)}=\frac{\partial p_{x}^{(i)}}{\partial \psi}\left(k_{H H}^{(i)}-\bar{k}_{H H}^{(i)}\right) \sin \alpha^{(i)} \cos \alpha^{(i)}+ \\
\frac{\partial p_{y}^{(i)}}{\partial \psi}\left(k_{H H}^{(i)} \cos ^{2} \alpha^{(i)}+\bar{k}_{H H}^{(i)} \sin ^{2} \alpha^{(i)}\right)=\frac{\partial p_{x}^{(i)}}{\partial \psi} K_{21}^{(i)}+\frac{\partial p_{y}^{(i)}}{\partial \psi} K_{22}^{(i)}
\end{aligned}
$$

- Third line coefficients - coefficients associated with a displacement $r_{z}$ :

$$
\begin{gathered}
K_{34}^{(i)}=-\frac{\partial p_{x}^{(i)}}{\partial \phi} k_{V H}^{(i)} \cos \alpha^{(i)}-\frac{\partial p_{y}^{(i)}}{\partial \phi} k_{V H}^{(i)} \sin \alpha^{(i)}+\frac{\partial p_{z}^{(i)}}{\partial \phi} k_{V V}^{(i)} \\
=\frac{\partial p_{x}^{(i)}}{\partial \phi} K_{31}^{(i)}+\frac{\partial p_{y}^{(i)}}{\partial \phi} K_{32}^{(i)}+\frac{\partial p_{z}^{(i)}}{\partial \phi} K_{33}^{(i)} \\
=\frac{\partial p_{x}^{(i)}}{\partial \theta} K_{31}^{(i)}+\frac{\partial p_{y}^{(i)}}{\partial \theta} K_{32}^{(i)}+\frac{\partial p_{z}^{(i)}}{\partial \theta} K_{33}^{(i)} \\
K_{35}^{(i)}=-\frac{\partial p_{x}^{(i)}}{\partial \theta} k_{V H}^{(i)} \cos \alpha^{(i)}-\frac{\partial p_{y}^{(i)}}{\partial \theta} k_{V H}^{(i)} \sin \alpha^{(i)}+\frac{\partial p_{z}^{(i)}}{\partial \theta} k_{V V}^{(i)} \\
K_{36}^{(i)}=-\frac{\partial p_{x}^{(i)}}{\partial \psi} k_{V H}^{(i)} \cos \alpha^{(i)}-\frac{\partial p_{y}^{(i)}}{\partial \psi} k_{V H}^{(i)} \sin \alpha^{(i)} \quad=\frac{\partial p_{x}^{(i)}}{\partial \psi} K_{31}^{(i)}+\frac{\partial p_{y}^{(i)}}{\partial \psi} K_{32}^{(i)}
\end{gathered}
$$

- Fourth line coefficients - coefficients associated with a displacement $\phi$ :

$$
\begin{aligned}
K_{41}^{(i)}= & \frac{\partial p_{x}^{(i)}}{\partial \phi}\left(k_{H H}^{(i)} \cos ^{2} \alpha^{(i)}+\bar{k}_{H H}^{(i)} \sin ^{2} \alpha^{(i)}\right)+ \\
& \frac{\partial p_{y}^{(i)}}{\partial \phi}\left(k_{H H}^{(i)}-\bar{k}_{H H}^{(i)}\right) \sin \alpha^{(i)} \cos \alpha^{(i)}-\frac{\partial p_{z}^{(i)}}{\partial \phi} k_{H V}^{(i)} \cos \alpha^{(i)} \\
= & \frac{\partial p_{x}^{(i)}}{\partial \phi} K_{11}^{(i)}+\frac{\partial p_{y}^{(i)}}{\partial \phi} K_{12}^{(i)}+\frac{\partial p_{z}^{(i)}}{\partial \phi} K_{13}^{(i)} \\
K_{42}^{(i)}= & \frac{\partial p_{x}^{(i)}}{\partial \phi}\left(k_{H H}^{(i)}-\bar{k}_{H H}^{(i)}\right) \sin \alpha^{(i)} \cos \alpha^{(i)}+ \\
& \frac{\partial p_{y}^{(i)}}{\partial \phi}\left(k_{H H}^{(i)} \cos ^{2} \alpha^{(i)}+\bar{k}_{H H}^{(i)} \sin ^{2} \alpha^{(i)}\right)-\frac{\partial p_{z}^{(i)}}{\partial \phi} k_{H V}^{(i)} \sin \alpha^{(i)} \\
= & \frac{\partial p_{x}^{(i)}}{\partial \phi} K_{21}^{(i)}+\frac{\partial p_{y}^{(i)}}{\partial \phi} K_{22}^{(i)}+\frac{\partial p_{z}^{(i)}}{\partial \phi} K_{23}^{(i)} \\
K_{43}^{(i)}=- & k_{H V}^{(i)}\left(\cos \alpha^{(i)} \frac{\partial p_{x}^{(i)}}{\partial \phi}+\sin \alpha^{(i)} \frac{\partial p_{y}^{(i)}}{\partial \phi}\right)+k_{V V}^{(i)} \frac{\partial p_{z}^{(i)}}{\partial \phi} \\
= & \frac{\partial p_{x}^{(i)}}{\partial \phi} K_{31}^{(i)}+\frac{\partial p_{y}^{(i)}}{\partial \phi} K_{32}^{(i)}+\frac{\partial p_{z}^{(i)}}{\partial \phi} K_{33}^{(i)}
\end{aligned}
$$




$$
\begin{aligned}
& K_{44}^{(i)}=\frac{\partial p_{x}^{(i)}}{\partial \phi} \frac{\partial p_{x}^{(i)}}{\partial \phi}\left(k_{H H}^{(i)} \cos ^{2} \alpha^{(i)}+\bar{k}_{H H}^{(i)} \sin ^{2} \alpha^{(i)}\right)+ \\
& \frac{\partial p_{x}^{(i)}}{\partial \phi} \frac{\partial p_{y}^{(i)}}{\partial \phi}\left(k_{H H}^{(i)}-\bar{k}_{H H}^{(i)}\right) \sin \alpha^{(i)} \cos \alpha^{(i)}-\frac{\partial p_{x}^{(i)}}{\partial \phi} \frac{\partial p_{z}^{(i)}}{\partial \phi} k_{H V}^{(i)} \cos \alpha^{(i)}+ \\
& \frac{\partial p_{y}^{(i)}}{\partial \phi} \frac{\partial p_{x}^{(i)}}{\partial \phi}\left(k_{H H}^{(i)}-\bar{k}_{H H}^{(i)}\right) \sin \alpha^{(i)} \cos \alpha^{(i)}+ \\
& \sum_{i=1}^{N}\left[\frac{\partial p_{y}^{(i)}}{\partial \phi} \frac{\partial p_{y}^{(i)}}{\partial \phi}\left(k_{H H}^{(i)} \sin ^{2} \alpha^{(i)}+\bar{k}_{H H}^{(i)} \cos ^{2} \alpha^{(i)}\right)-\frac{\partial p_{y}^{(i)}}{\partial \phi} \frac{\partial p_{z}^{(i)}}{\partial \phi} k_{H V}^{(i)} \sin \alpha^{(i)}\right]+ \\
& \sum_{i=1}^{N}\left[-\frac{\partial p_{z}^{(i)}}{\partial \phi} \frac{\partial p_{x}^{(i)}}{\partial \phi} k_{H V}^{(i)} \cos \alpha^{(i)}-\frac{\partial p_{z}^{(i)}}{\partial \phi} \frac{\partial p_{y}^{(i)}}{\partial \phi} k_{H V}^{(i)} \sin \alpha^{(i)}+\frac{\partial p_{z}^{(i)}}{\partial \phi} \frac{\partial p_{z}^{(i)}}{\partial \phi} k_{V V}^{(i)}\right]+ \\
& \quad-\frac{\partial^{2} p_{x}^{(i)}}{\partial \phi^{2}} F_{H}^{(i)} \cos \alpha^{(i)}-\frac{\partial^{2} p_{y}^{(i)}}{\partial \phi^{2}} F_{H}^{(i)} \sin \alpha^{(i)}-\frac{\partial^{2} p_{z}^{(i)}}{\partial \phi^{2}} F_{V}^{(i)} \\
& \frac{\partial p_{x}^{(i)}}{\partial \phi} K_{14}^{(i)}+\frac{\partial p_{y}^{(i)}}{\partial \phi} K_{24}^{(i)}+\frac{\partial p_{z}^{(i)}}{\partial \phi} K_{34}^{(i)}- \\
& \frac{\partial^{2} p_{x}^{(i)}}{\partial \phi^{2}} F_{H}^{(i)} \cos \alpha^{(i)}-\frac{\partial^{2} p_{y}^{(i)}}{\partial \phi^{2}} F_{H}^{(i)} \sin \alpha^{(i)}+\frac{\partial^{2} p_{z}^{(i)}}{\partial \phi^{2}} F_{V}^{(i)} \quad(\mathrm{E} .13)
\end{aligned}
$$

$$
\begin{aligned}
& K_{45}^{(i)}=\frac{\partial p_{x}^{(i)}}{\partial \phi} \frac{\partial p_{x}^{(i)}}{\partial \theta}\left(k_{H H}^{(i)} \cos ^{2} \alpha^{(i)}+\bar{k}_{H H}^{(i)} \sin ^{2} \alpha^{(i)}\right)+ \\
& \frac{\partial p_{x}^{(i)}}{\partial \phi} \frac{\partial p_{y}^{(i)}}{\partial \theta}\left(k_{H H}^{(i)}-\bar{k}_{H H}^{(i)}\right) \sin \alpha^{(i)} \cos \alpha^{(i)}-\frac{\partial p_{x}^{(i)}}{\partial \phi} \frac{\partial p_{z}^{(i)}}{\partial \theta} k_{H V}^{(i)} \cos \alpha^{(i)}+ \\
& \frac{\partial p_{y}^{(i)}}{\partial \phi} \frac{\partial p_{x}^{(i)}}{\partial \theta}\left(k_{H H}^{(i)}-\bar{k}_{H H}^{(i)}\right) \sin \alpha^{(i)} \cos \alpha^{(i)}+ \\
& \frac{\partial p_{y}^{(i)}}{\partial \phi} \frac{\partial p_{y}^{(i)}}{\partial \theta}\left(k_{H H}^{(i)} \sin ^{2} \alpha^{(i)}+\bar{k}_{H H}^{(i)} \cos ^{2} \alpha^{(i)}\right)-\frac{\partial p_{y}^{(i)}}{\partial \phi} \frac{\partial p_{z}^{(i)}}{\partial \theta} k_{H V}^{(i)} \sin \alpha^{(i)}+ \\
& -\frac{\partial p_{z}^{(i)}}{\partial \phi} \frac{\partial p_{x}^{(i)}}{\partial \theta} k_{H V}^{(i)} \cos \alpha^{(i)}-\frac{\partial p_{z}^{(i)}}{\partial \phi} \frac{\partial p_{y}^{(i)}}{\partial \theta} k_{H V}^{(i)} \sin \alpha^{(i)}+\frac{\partial p_{z}^{(i)}}{\partial \phi} \frac{\partial p_{z}^{(i)}}{\partial \theta} k_{V V}^{(i)}+ \\
& -\frac{\partial^{2} p_{x}^{(i)}}{\partial \phi \partial \theta} F_{H} \cos \alpha^{(i)}-\frac{\partial^{2} p_{y}^{(i)}}{\partial \phi \partial \theta} F_{H} \sin \alpha^{(i)}+\frac{\partial^{2} p_{z}^{(i)}}{\partial \phi \partial \theta} F_{V} \\
& =\frac{\partial p_{x}^{(i)}}{\partial \phi} K_{15}^{(i)}+\frac{\partial p_{y}^{(i)}}{\partial \phi} K_{25}^{(i)}+\frac{\partial p_{z}^{(i)}}{\partial \phi} K_{35}^{(i)}- \\
& \frac{\partial^{2} p_{x}^{(i)}}{\partial \phi \partial \theta} F_{H}^{(i)} \cos \alpha^{(i)}-\frac{\partial^{2} p_{y}^{(i)}}{\partial \phi \partial \theta} F_{H}^{(i)} \sin \alpha^{(i)}+\frac{\partial^{2} p_{z}^{(i)}}{\partial \phi \partial \theta} F_{V}^{(i)}
\end{aligned}
$$




$$
\begin{aligned}
& K_{46}^{(i)}=\frac{\partial p_{x}^{(i)}}{\partial \phi} \frac{\partial p_{x}^{(i)}}{\partial \psi}\left(k_{H H}^{(i)} \cos ^{2} \alpha^{(i)}+\bar{k}_{H H}^{(i)} \sin ^{2} \alpha^{(i)}\right)+ \\
& \frac{\partial p_{x}^{(i)}}{\partial \phi} \frac{\partial p_{y}^{(i)}}{\partial \psi}\left(k_{H H}^{(i)}-\bar{k}_{H H}^{(i)}\right) \sin \alpha^{(i)} \cos \alpha^{(i)}-\frac{\partial p_{x}^{(i)}}{\partial \phi} \frac{\partial p_{z}^{(i)}}{\partial \psi} k_{H V}^{(i)} \cos \alpha^{(i)}+ \\
& \frac{\partial p_{y}^{(i)}}{\partial \phi} \frac{\partial p_{x}^{(i)}}{\partial \psi}\left(k_{H H}^{(i)}-\bar{k}_{H H}^{(i)}\right) \sin \alpha^{(i)} \cos \alpha^{(i)}+ \\
& \frac{\partial p_{y}^{(i)}}{\partial \phi} \frac{\partial p_{y}^{(i)}}{\partial \psi}\left(k_{H H}^{(i)} \sin ^{2} \alpha^{(i)}+\bar{k}_{H H}^{(i)} \cos ^{2} \alpha^{(i)}\right)-\frac{\partial p_{y}^{(i)}}{\partial \phi} \frac{\partial p_{z}^{(i)}}{\partial \psi} k_{H V}^{(i)} \sin \alpha^{(i)}+ \\
& -\frac{\partial p_{z}^{(i)}}{\partial \phi} \frac{\partial p_{x}^{(i)}}{\partial \psi} k_{H V}^{(i)} \cos \alpha^{(i)}-\frac{\partial p_{z}^{(i)}}{\partial \phi} \frac{\partial p_{y}^{(i)}}{\partial \psi} k_{H V}^{(i)} \sin \alpha^{(i)}+\frac{\partial p_{z}^{(i)}}{\partial \phi} \frac{\partial p_{z}^{(i)}}{\partial \psi} k_{V V}^{(i)}+ \\
& -\frac{\partial^{2} p_{x}^{(i)}}{\partial \phi \partial \psi} F_{H} \cos \alpha^{(i)}-\frac{\partial^{2} p_{y}^{(i)}}{\partial \phi \partial \psi} F_{H} \sin \alpha^{(i)}+\frac{\partial^{2} p_{z}^{(i)}}{\partial \phi \partial \psi} F_{V} \\
& =\frac{\partial p_{x}^{(i)}}{\partial \phi} K_{16}^{(i)}+\frac{\partial p_{y}^{(i)}}{\partial \phi} K_{26}^{(i)}+\frac{\partial p_{z}^{(i)}}{\partial \phi} K_{36}^{(i)}- \\
& \frac{\partial^{2} p_{x}^{(i)}}{\partial \phi \partial \psi} F_{H}^{(i)} \cos \alpha^{(i)}-\frac{\partial^{2} p_{y}^{(i)}}{\partial \phi \partial \psi} F_{H}^{(i)} \sin \alpha^{(i)}+\frac{\partial^{2} p_{z}^{(i)}}{\partial \phi \partial \psi} F_{V}^{(i)}
\end{aligned}
$$

- Fifth line coefficients - coefficients associated with a displacement $\theta$ :

$$
\begin{aligned}
K_{51}^{(i)}=\frac{\partial p_{x}^{(i)}}{\partial \theta}\left(k_{H H}^{(i)} \cos ^{2} \alpha^{(i)}+\bar{k}_{H H}^{(i)} \sin ^{2} \alpha^{(i)}\right) & + \\
\frac{\partial p_{y}^{(i)}}{\partial \theta}\left(k_{H H}^{(i)}-\bar{k}_{H H}^{(i)}\right) \sin \alpha^{(i)} \cos \alpha^{(i)}-\frac{\partial p_{z}^{(i)}}{\partial \theta} k_{H V}^{(i)} \cos \alpha^{(i)} & \\
= & \frac{\partial p_{x}^{(i)}}{\partial \theta} K_{11}^{(i)}+\frac{\partial p_{y}^{(i)}}{\partial \theta} K_{12}^{(i)}+\frac{\partial p_{z}^{(i)}}{\partial \theta} K_{13}^{(i)}
\end{aligned}
$$

$$
\begin{aligned}
K_{52}^{(i)}= & \frac{\partial p_{x}^{(i)}}{\partial \theta}\left(k_{H H}^{(i)}-\bar{k}_{H H}^{(i)}\right) \sin \alpha^{(i)} \cos \alpha^{(i)}+ \\
\frac{\partial p_{y}^{(i)}}{\partial \theta}\left(k_{H H}^{(i)} \cos ^{2} \alpha^{(i)}+\bar{k}_{H H}^{(i)} \sin ^{2} \alpha^{(i)}\right)-\frac{\partial p_{z}^{(i)}}{\partial \theta} k_{H V}^{(i)} \sin \alpha^{(i)} & \\
& =\frac{\partial p_{x}^{(i)}}{\partial \theta} K_{21}^{(i)}+\frac{\partial p_{y}^{(i)}}{\partial \theta} K_{22}^{(i)}+\frac{\partial p_{z}^{(i)}}{\partial \theta} K_{23}^{(i)}
\end{aligned}
$$

$$
\begin{aligned}
K_{53}^{(i)}=-k_{H V}^{(i)}\left(\cos \alpha^{(i)} \frac{\partial p_{x}^{(i)}}{\partial \theta}+\sin \alpha^{(i)} \frac{\partial p_{y}^{(i)}}{\partial \theta}\right)+k_{V V}^{(i)} \frac{\partial p_{z}^{(i)}}{\partial \theta} & \\
& =\frac{\partial p_{x}^{(i)}}{\partial \theta} K_{31}^{(i)}+\frac{\partial p_{y}^{(i)}}{\partial \theta} K_{32}^{(i)}+\frac{\partial p_{z}^{(i)}}{\partial \theta} K_{33}^{(i)}
\end{aligned}
$$




$$
\begin{aligned}
& K_{54}^{(i)}=\frac{\partial p_{x}^{(i)}}{\partial \theta} \frac{\partial p_{x}^{(i)}}{\partial \phi}\left(k_{H H}^{(i)} \cos ^{2} \alpha^{(i)}+\bar{k}_{H H}^{(i)} \sin ^{2} \alpha^{(i)}\right)+ \\
& \frac{\partial p_{x}^{(i)}}{\partial \theta} \frac{\partial p_{y}^{(i)}}{\partial \phi}\left(k_{H H}^{(i)}-\bar{k}_{H H}^{(i)}\right) \sin \alpha^{(i)} \cos \alpha^{(i)}-\frac{\partial p_{x}^{(i)}}{\partial \theta} \frac{\partial p_{z}^{(i)}}{\partial \phi} k_{H V}^{(i)} \cos \alpha^{(i)}+ \\
& \frac{\partial p_{y}^{(i)}}{\partial \theta} \frac{\partial p_{x}^{(i)}}{\partial \phi}\left(k_{H H}^{(i)}-\bar{k}_{H H}^{(i)}\right) \sin \alpha^{(i)} \cos \alpha^{(i)}+ \\
& \frac{\partial p_{y}^{(i)}}{\partial \theta} \frac{\partial p_{y}^{(i)}}{\partial \phi}\left(k_{H H}^{(i)} \sin ^{2} \alpha^{(i)}+\bar{k}_{H H}^{(i)} \cos ^{2} \alpha^{(i)}\right)-\frac{\partial p_{y}^{(i)}}{\partial \theta} \frac{\partial p_{z}^{(i)}}{\partial \phi} k_{H V}^{(i)} \sin \alpha^{(i)}+ \\
& -\frac{\partial p_{z}^{(i)}}{\partial \theta} \frac{\partial p_{x}^{(i)}}{\partial \phi} k_{H V}^{(i)} \cos \alpha^{(i)}-\frac{\partial p_{z}^{(i)}}{\partial \theta} \frac{\partial p_{y}^{(i)}}{\partial \phi} k_{H V}^{(i)} \sin \alpha^{(i)}+\frac{\partial p_{z}^{(i)}}{\partial \theta} \frac{\partial p_{z}^{(i)}}{\partial \phi} k_{V V}^{(i)}- \\
& \quad-\frac{\partial^{2} p_{x}^{(i)}}{\partial \theta \partial \phi} F_{H} \cos \alpha^{(i)}-\frac{\partial^{2} p_{y}^{(i)}}{\partial \theta \partial \phi} F_{H} \sin \alpha^{(i)}+\frac{\partial^{2} p_{z}^{(i)}}{\partial \theta \partial \phi} F_{V} \\
& =\frac{\partial p_{x}^{(i)}}{\partial \theta} K_{14}^{(i)}+\frac{\partial p_{y}^{(i)}}{\partial \theta} K_{24}^{(i)}+\frac{\partial p_{z}^{(i)}}{\partial \theta} K_{34}^{(i)} \\
& -\frac{\partial^{2} p_{x}^{(i)}}{\partial \theta \partial \phi} F_{H}^{(i)} \cos \alpha^{(i)}-\frac{\partial^{2} p_{y}^{(i)}}{\partial \theta \partial \phi} F_{H}^{(i)} \sin \alpha^{(i)}+\frac{\partial^{2} p_{z}^{(i)}}{\partial \theta \partial \phi} F_{V}^{(i)}
\end{aligned}
$$

$$
\begin{aligned}
& K_{55}^{(i)}=\frac{\partial p_{x}^{(i)}}{\partial \theta} \frac{\partial p_{x}^{(i)}}{\partial \theta}\left(k_{H H}^{(i)} \cos ^{2} \alpha^{(i)}+\bar{k}_{H H}^{(i)} \sin ^{2} \alpha^{(i)}\right) \\
& \frac{\partial p_{x}^{(i)}}{\partial \theta} \frac{\partial p_{y}^{(i)}}{\partial \theta}\left(k_{H H}^{(i)}-\bar{k}_{H H}^{(i)}\right) \sin \alpha^{(i)} \cos \alpha^{(i)}-\frac{\partial p_{x}^{(i)}}{\partial \theta} \frac{\partial p_{z}^{(i)}}{\partial \theta} k_{H V}^{(i)} \cos \alpha^{(i)}+ \\
& \frac{\partial p_{y}^{(i)}}{\partial \theta} \frac{\partial p_{x}^{(i)}}{\partial \theta}\left(k_{H H}^{(i)}-\bar{k}_{H H}^{(i)}\right) \sin \alpha^{(i)} \cos \alpha^{(i)} \\
& \quad \frac{\partial p_{y}^{(i)}}{\partial \theta} \frac{\partial p_{y}^{(i)}}{\partial \theta}\left(k_{H H}^{(i)} \sin ^{2} \alpha^{(i)}+\bar{k}_{H H}^{(i)} \cos ^{2} \alpha^{(i)}\right)-\frac{\partial p_{y}^{(i)}}{\partial \theta} \frac{\partial p_{z}^{(i)}}{\partial \theta} k_{H V}^{(i)} \sin \alpha^{(i)}+ \\
& \quad-\frac{\partial p_{z}^{(i)}}{\partial \theta} \frac{\partial p_{x}^{(i)}}{\partial \theta} k_{H V}^{(i)} \cos \alpha^{(i)}-\frac{\partial p_{z}^{(i)}}{\partial \theta} \frac{\partial p_{y}^{(i)}}{\partial \theta} k_{H V}^{(i)} \sin \alpha^{(i)}+\frac{\partial p_{z}^{(i)}}{\partial \theta} \frac{\partial p_{z}^{(i)}}{\partial \theta} k_{V V}^{(i)}+ \\
& \quad-\frac{\partial^{2} p_{x}^{(i)}}{\partial \theta^{2}} F_{H} \cos \alpha^{(i)}-\frac{\partial^{2} p_{y}^{(i)}}{\partial \theta^{2}} F_{H} \sin \alpha^{(i)}+\frac{\partial^{2} p_{z}^{(i)}}{\partial \theta^{2}} F_{V} \\
& =\frac{\partial p_{x}^{(i)}}{\partial \theta} K_{15}^{(i)}+\frac{\partial p_{y}^{(i)}}{\partial \theta} K_{25}^{(i)}+\frac{\partial p_{z}^{(i)}}{\partial \theta} K_{35}^{(i)} \\
& -\frac{\partial^{2} p_{x}^{(i)}}{\partial \theta^{2}} F_{H}^{(i)} \cos \alpha^{(i)}-\frac{\partial^{2} p_{y}^{(i)}}{\partial \theta^{2}} F_{H}^{(i)} \sin \alpha^{(i)}+\frac{\partial^{2} p_{z}^{(i)}}{\partial \theta^{2}} F_{V}^{(i)}
\end{aligned}
$$




$$
\begin{aligned}
& K_{56}^{(i)}=\frac{\partial p_{x}^{(i)}}{\partial \theta} \frac{\partial p_{x}^{(i)}}{\partial \psi}\left(k_{H H}^{(i)} \cos ^{2} \alpha^{(i)}+\bar{k}_{H H}^{(i)} \sin ^{2} \alpha^{(i)}\right) \\
& \frac{\partial p_{x}^{(i)}}{\partial \theta} \frac{\partial p_{y}^{(i)}}{\partial \psi}\left(k_{H H}^{(i)}-\bar{k}_{H H}^{(i)}\right) \sin \alpha^{(i)} \cos \alpha^{(i)}-\frac{\partial p_{x}^{(i)}}{\partial \theta} \frac{\partial p_{z}^{(i)}}{\partial \psi} k_{H V}^{(i)} \cos \alpha^{(i)}+ \\
& \quad \frac{\partial p_{y}^{(i)}}{\partial \theta} \frac{\partial p_{x}^{(i)}}{\partial \psi}\left(k_{H H}^{(i)}-\bar{k}_{H H}^{(i)}\right) \sin \alpha^{(i)} \cos \alpha^{(i)}+ \\
& \frac{\partial p_{y}^{(i)}}{\partial \theta} \frac{\partial p_{y}^{(i)}}{\partial \psi}\left(k_{H H}^{(i)} \sin ^{2} \alpha^{(i)}+\bar{k}_{H H}^{(i)} \cos ^{2} \alpha^{(i)}\right)-\frac{\partial p_{y}^{(i)}}{\partial \theta} \frac{\partial p_{z}^{(i)}}{\partial \psi} k_{H V}^{(i)} \sin \alpha^{(i)}+ \\
& \quad-\frac{\partial p_{z}^{(i)}}{\partial \theta} \frac{\partial p_{x}^{(i)}}{\partial \psi} k_{H V}^{(i)} \cos \alpha^{(i)}-\frac{\partial p_{z}^{(i)}}{\partial \theta} \frac{\partial p_{y}^{(i)}}{\partial \psi} k_{H V}^{(i)} \sin \alpha^{(i)}+\frac{\partial p_{z}^{(i)}}{\partial \theta} \frac{\partial p_{z}^{(i)}}{\partial \psi} k_{V V}^{(i)}+ \\
& \quad-\frac{\partial^{2} p_{x}^{(i)}}{\partial \theta \partial \psi} F_{H} \cos \alpha^{(i)}-\frac{\partial^{2} p_{y}^{(i)}}{\partial \theta \partial \psi} F_{H} \sin \alpha^{(i)}+\frac{\partial^{2} p_{z}^{(i)}}{\partial \theta \partial \psi} F_{V} \\
& =\frac{\partial p_{x}^{(i)}}{\partial \theta} K_{16}^{(i)}+\frac{\partial p_{y}^{(i)}}{\partial \theta} K_{26}^{(i)}+\frac{\partial p_{z}^{(i)}}{\partial \theta} K_{36}^{(i)} \\
& -\frac{\partial^{2} p_{x}^{(i)}}{\partial \theta \partial \psi} F_{H}^{(i)} \cos \alpha^{(i)}-\frac{\partial^{2} p_{y}^{(i)}}{\partial \theta \partial \psi} F_{H}^{(i)} \sin \alpha^{(i)}+\frac{\partial^{2} p_{z}^{(i)}}{\partial \theta \partial \psi} F_{V}^{(i)}
\end{aligned}
$$

- Sixth line coefficients - coefficients associated with a displacement $\psi$ :

$$
\begin{aligned}
K_{61}^{(i)}=\frac{\partial p_{x}^{(i)}}{\partial \psi}\left(k_{H H}^{(i)} \cos ^{2} \alpha^{(i)}+\bar{k}_{H H}^{(i)} \sin ^{2} \alpha^{(i)}\right)+ & \\
\frac{\partial p_{y}^{(i)}}{\partial \psi}\left(k_{H H}^{(i)}-\bar{k}_{H H}^{(i)}\right) \sin \alpha^{(i)} \cos \alpha^{(i)} & =\frac{\partial p_{x}^{(i)}}{\partial \psi} K_{11^{(i)}}+\frac{\partial p_{y}^{(i)}}{\partial \psi} K_{12}^{(i)}
\end{aligned}
$$

$$
\begin{aligned}
K_{62}^{(i)}=\frac{\partial p_{x}^{(i)}}{\partial \psi}\left(k_{H H}^{(i)}-\bar{k}_{H H}^{(i)}\right) \sin \alpha^{(i)} \cos \alpha^{(i)} & \\
\frac{\partial p_{y}^{(i)}}{\partial \psi}\left(k_{H H}^{(i)} \cos ^{2} \alpha^{(i)}+\bar{k}_{H H}^{(i)} \sin ^{2} \alpha^{(i)}\right) & =\frac{\partial p_{x}^{(i)}}{\partial \psi} K_{21}^{(i)}+\frac{\partial p_{y}^{(i)}}{\partial \psi} K_{22}^{(i)}
\end{aligned}
$$

$$
K_{63}^{(i)}=-k_{H V}^{(i)}\left(\cos \alpha^{(i)} \frac{\partial p_{x}^{(i)}}{\partial \psi}+\sin \alpha^{(i)} \frac{\partial p_{y}^{(i)}}{\partial \psi}\right)=\frac{\partial p_{x}^{(i)}}{\partial \psi} K_{31}^{(i)}+\frac{\partial p_{y}^{(i)}}{\partial \psi} K_{32}^{(i)}
$$




$$
\begin{aligned}
& K_{64}^{(i)}=\frac{\partial p_{x}^{(i)}}{\partial \psi} \frac{\partial p_{x}^{(i)}}{\partial \phi}\left(k_{H H}^{(i)} \cos ^{2} \alpha^{(i)}+\bar{k}_{H H}^{(i)} \sin ^{2} \alpha^{(i)}\right)+ \\
& \frac{\partial p_{x}^{(i)}}{\partial \psi} \frac{\partial p_{y}^{(i)}}{\partial \phi}\left(k_{H H}^{(i)}-\bar{k}_{H H}^{(i)}\right) \sin \alpha^{(i)} \cos \alpha^{(i)}+\frac{\partial p_{x}^{(i)}}{\partial \psi} \frac{\partial p_{z}^{(i)}}{\partial \phi} k_{H V}^{(i)} \cos \alpha^{(i)}+ \\
& \frac{\partial p_{y}^{(i)}}{\partial \psi} \frac{\partial p_{x}^{(i)}}{\partial \phi}\left(k_{H H}^{(i)}-\bar{k}_{H H}^{(i)}\right) \sin \alpha^{(i)} \cos \alpha^{(i)} \\
& \frac{\partial p_{y}^{(i)}}{\partial \psi} \frac{\partial p_{y}^{(i)}}{\partial \phi}\left(k_{H H}^{(i)} \sin ^{2} \alpha^{(i)}+\bar{k}_{H H}^{(i)} \cos ^{2} \alpha^{(i)}\right)+\frac{\partial p_{y}^{(i)}}{\partial \psi} \frac{\partial p_{z}^{(i)}}{\partial \phi} k_{H V}^{(i)} \sin \alpha^{(i)}+ \\
& -\frac{\partial^{2} p_{x}^{(i)}}{\partial \psi \partial \phi} F_{H} \cos \alpha^{(i)}-\frac{\partial^{2} p_{y}^{(i)}}{\partial \psi \partial \phi} F_{H} \sin \alpha^{(i)} \\
& =\frac{\partial p_{x}^{(i)}}{\partial \psi} K_{14}^{(i)}+\frac{\partial p_{y}^{(i)}}{\partial \psi} K_{24}^{(i)}-\frac{\partial^{2} p_{x}^{(i)}}{\partial \psi \partial \phi} F_{H}^{(i)} \cos \alpha^{(i)}-\frac{\partial^{2} p_{y}^{(i)}}{\partial \psi \partial \phi} F_{H}^{(i)} \sin \alpha^{(i)} \\
& K_{65}^{(i)}=\frac{\partial p_{x}^{(i)}}{\partial \psi} \frac{\partial p_{x}^{(i)}}{\partial \theta}\left(k_{H H}^{(i)} \cos ^{2} \alpha^{(i)}+\bar{k}_{H H}^{(i)} \sin ^{2} \alpha^{(i)}\right) \\
& \frac{\partial p_{x}^{(i)}}{\partial \psi} \frac{\partial p_{y}^{(i)}}{\partial \theta}\left(k_{H H}^{(i)}-\bar{k}_{H H}^{(i)}\right) \sin \alpha^{(i)} \cos \alpha^{(i)}+\frac{\partial p_{x}^{(i)}}{\partial \psi} \frac{\partial p_{z}^{(i)}}{\partial \theta} k_{H V}^{(i)} \cos \alpha^{(i)}+ \\
& \frac{\partial p_{y}^{(i)}}{\partial \psi} \frac{\partial p_{x}^{(i)}}{\partial \theta}\left(k_{H H}^{(i)}-\bar{k}_{H H}^{(i)}\right) \sin \alpha^{(i)} \cos \alpha^{(i)}+ \\
& \frac{\partial p_{y}^{(i)}}{\partial \psi} \frac{\partial p_{y}^{(i)}}{\partial \theta}\left(k_{H H}^{(i)} \sin ^{2} \alpha^{(i)}+\bar{k}_{H H}^{(i)} \cos ^{2} \alpha^{(i)}\right)+\frac{\partial p_{y}^{(i)}}{\partial \psi} \frac{\partial p_{z}^{(i)}}{\partial \theta} k_{H V}^{(i)} \sin \alpha^{(i)}+ \\
& -\frac{\partial^{2} p_{x}^{(i)}}{\partial \psi \partial \theta} F_{H} \cos \alpha^{(i)}-\frac{\partial^{2} p_{y}^{(i)}}{\partial \psi \partial \theta} F_{H} \sin \alpha^{(i)} \\
& =\frac{\partial p_{x}^{(i)}}{\partial \psi} K_{15}^{(i)}+\frac{\partial p_{y}^{(i)}}{\partial \psi} K_{25}^{(i)}-\frac{\partial^{2} p_{x}^{(i)}}{\partial \psi \partial \theta} F_{H}^{(i)} \cos \alpha^{(i)}-\frac{\partial^{2} p_{y}^{(i)}}{\partial \psi \partial \theta} F_{H}^{(i)} \sin \alpha^{(i)} \\
& K_{66}^{(i)}=\frac{\partial p_{x}^{(i)}}{\partial \psi} \frac{\partial p_{x}^{(i)}}{\partial \psi}\left(k_{H H}^{(i)} \cos ^{2} \alpha^{(i)}+\bar{k}_{H H}^{(i)} \sin ^{2} \alpha^{(i)}\right)+ \\
& \frac{\partial p_{x}^{(i)}}{\partial \psi} \frac{\partial p_{y}^{(i)}}{\partial \psi}\left(k_{H H}^{(i)}-\bar{k}_{H H}^{(i)}\right) \sin \alpha^{(i)} \cos \alpha^{(i)}+ \\
& \frac{\partial p_{y}^{(i)}}{\partial \psi} \frac{\partial p_{x}^{(i)}}{\partial \psi}\left(k_{H H}^{(i)}-\bar{k}_{H H}^{(i)}\right) \sin \alpha^{(i)} \cos \alpha^{(i)}+ \\
& \frac{\partial p_{y}^{(i)}}{\partial \psi} \frac{\partial p_{y}^{(i)}}{\partial \psi}\left(k_{H H}^{(i)} \sin ^{2} \alpha^{(i)}+\bar{k}_{H H}^{(i)} \cos ^{2} \alpha^{(i)}\right)+ \\
& -\frac{\partial^{2} p_{x}^{(i)}}{\partial \psi^{2}} F_{H} \cos \alpha^{(i)}-\frac{\partial^{2} p_{y}^{(i)}}{\partial \psi^{2}} F_{H} \sin \alpha^{(i)} \\
& =\frac{\partial p_{x}^{(i)}}{\partial \psi} K_{14}^{(i)}+\frac{\partial p_{y}^{(i)}}{\partial \psi} K_{24}^{(i)}-\frac{\partial^{2} p_{x}^{(i)}}{\partial \psi^{2}} F_{H}^{(i)} \cos \alpha^{(i)}-\frac{\partial^{2} p_{y}^{(i)}}{\partial \psi^{2}} F_{H}^{(i)} \sin \alpha^{(i)}
\end{aligned}
$$





\section{APPENDIX F - Stiffness matrix for the planar problem}

Sometimes, specially during early stages of the mooring system design, the designer may be interested only on the problem related to motions on the horizontal plane Oxy. This could be justified by the fact that natural periods of motions on the horizontal plane $-r_{x}, r_{y}$ and $\psi$ - have values of a different order of magnitude compared to those obtained by others DoFs. The objective of the present Appendix is to simplify the obtained stiffness matrix in order to determine the horizontal-plane problem. In this scenario, results are compared with those published in Pesce, Amaral and Franzini (2018).

Consider then a moored floating body in a generic offset position and heading. Figure 66 recovers the sketch presented in Pesce, Amaral and Franzini (2018). The projection of the anchors and fairleads position onto the plane $\pi_{h}$ is detailed for a particular mooring line $i=1, \ldots, N$.

Figure 66 - Sketch of the projection of a moored floating body in a generic offset position and heading onto the horizontal plane $\pi$.

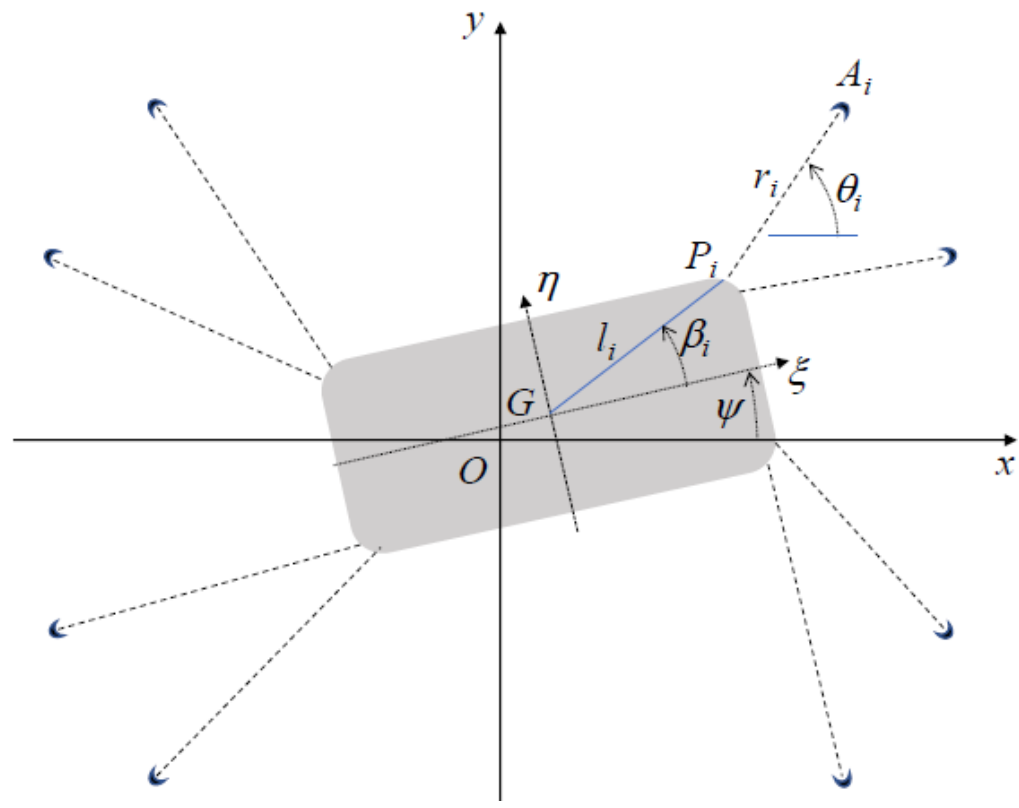

Source: Pesce, Amaral and Franzini (2018)

By defining $\beta^{(i)}$ as the angle formed by $\left(\left\{\vec{P}_{i}\right\}_{E_{\xi}}^{\pi}-G\right)$ with respect to the axis $G \xi$ and $l^{(i)}$ and the distance between $\left\{\vec{P}_{i}\right\}_{E_{\xi}}^{\pi_{h}}$ and $G$ (as in Pesce, Amaral and Franzini (2018)) and considering, without losing generalizations, that $p_{\zeta}^{(i)}=0$, the position $\left\{\vec{P}_{i}\right\}_{E_{\xi}}^{\pi}$ 
is written as:

$$
\left\{\vec{P}_{i}\right\}_{E_{\xi}}^{\pi_{h}}=\left(\begin{array}{lll}
p_{\xi}^{(i)} & p_{\eta}^{(i)} & p_{\zeta}^{(i)}
\end{array}\right)^{t}=\left(l^{(i)} \cos \alpha^{(i)} \quad l^{(i)} \sin \alpha^{(i)} \quad 0\right)^{t}
$$

Let us consider now that the vessel describes a motion only on plane $\pi_{h}$. In other words, one can consider that generalized translation $r_{z}$ and generalized rotations $\phi$ and $\theta$ are null. In this case, it is possible to rewrite the rotation matrix $[\mathbb{R}]_{E_{x} \mid E_{\xi}}$ as:

$$
[\mathbb{R}]_{E_{x} \mid E_{\xi}}^{\pi}=\left(\begin{array}{ccc}
\cos \psi & -\sin \psi & 0 \\
\sin \psi & \cos \psi & 0 \\
0 & 0 & 1
\end{array}\right)
$$

Consequently, the position of each fairlead with respect to the fixed frame $E_{x}$ becomes:

$$
\begin{aligned}
\left\{\vec{P}_{i}\right\}_{E_{x}} \pi_{h}=\left(\begin{array}{lll}
r_{x}+p_{\xi}^{(i)} \cos \psi-p_{\eta}^{(i)} \sin \psi & r_{y}+p_{\xi}^{(i)} \sin \psi+p_{\eta}^{(i)} \cos \psi & 0
\end{array}\right)^{t}= \\
\left(\begin{array}{lll}
r_{x}+l^{(i)} \cos \left(\psi+\beta^{(i)}\right) & r_{y}+l^{(i)} \sin \left(\psi+\beta^{(i)}\right) & 0
\end{array}\right)^{t}
\end{aligned}
$$

Thus, the horizontal anchor-fairlead distance is rewritten:

$$
r^{(i)}=\sqrt{\left[a_{x}^{(i)}-\left(r_{x}+l^{(i)} \cos \left(\psi+\beta^{(i)}\right)\right)\right]^{2}+\left[a_{y}^{(i)}-\left(r_{y}+l^{(i)} \sin \left(\psi+\beta^{(i)}\right)\right)\right]^{2}}
$$

Then, the directional angle $\alpha^{(i)}$ that defines the directional unity vector $\hat{e}_{h}^{(i)}$ reads:

$$
\begin{aligned}
\cos \alpha^{(i)} & =\frac{a_{x}^{(i)}-\left(r_{x}+l^{(i)} \cos \left(\psi+\beta^{(i)}\right)\right)}{r^{(i)}} \\
\sin \alpha^{(i)} & =\frac{a_{y}^{(i)}-\left(r_{y}+l^{(i)} \sin \left(\psi+\beta^{(i)}\right)\right)}{r^{(i)}}
\end{aligned}
$$

Now, it is easy to notice that the stiffness coefficients associated with the translational displacements proposed in Pesce, Amaral and Franzini (2018) define a particular case of the methodology herein proposed, when the planar problem is considered.

Before verifying how the translation-rotation and rotation-rotation coefficients of the stiffness matrix are turned, it is necessary to determine the derivatives of $\left\{\vec{P}_{i}\right\}_{E_{x}}^{\pi}$ with respect to $\psi$ and the respective second derivatives, also with respect to $\psi$.

$$
\begin{aligned}
& \frac{\partial p_{x}^{(i)}}{\partial \psi}=-l^{(i)} \sin \left(\psi+\beta^{(i)}\right) \\
& \frac{\partial p_{y}^{(i)}}{\partial \psi}=l^{(i)} \cos \left(\psi+\beta^{(i)}\right) \\
& \frac{\partial^{2} p_{x}^{(i)}}{\partial \psi^{2}}=-l^{(i)} \cos \left(\psi+\beta^{(i)}\right) \\
& \frac{\partial^{2} p_{y}^{(i)}}{\partial \psi^{2}}=-l^{(i)} \sin \left(\psi+\beta^{(i)}\right)
\end{aligned}
$$


Now, it is possible to rewrite the lacking planar stiffness coefficients, i.e., $K_{16}, K_{26}$, $K_{61}, K_{62}$ and $K_{66}$. Additionally, due to the aforementioned symmetry of the stiffness matrix, $K_{16}=K_{61}$ and $K_{26}=K_{62}$

$$
\begin{aligned}
& K_{16}=\sum_{i=1}^{N}\left(\frac{\partial p_{x}^{(i)}}{\partial \psi} K_{11}+\frac{\partial p_{y}^{(i)}}{\partial \psi} K_{11}\right)= \\
& -l^{(i)} \sin \left(\psi+\beta^{(i)}\right) K_{11}+l^{(i)} \cos \left(\psi+\beta^{(i)}\right) K_{12}= \\
& -l^{(i)} \sin \left(\psi+\beta^{(i)}\right)\left(k_{H H}^{(i)} \cos ^{2} \alpha^{(i)}+\bar{k}_{H H}^{(i)} \sin ^{2} \alpha^{(i)}\right)+ \\
& \quad l^{(i)} \cos \left(\psi+\beta^{(i)}\right)\left(k_{H H}^{(i)}-\bar{k}_{H H}^{(i)}\right) \cos \alpha^{(i)} \sin \alpha^{(i)}= \\
& \quad k_{H H} l^{(i)} \cos \alpha^{(i)} \sin \left(\alpha^{(i)}-\beta^{(i)}-\psi\right)-\bar{k}_{H H} l^{(i)} \sin \alpha^{(i)} \cos \left(\alpha^{(i)}-\beta^{(i)}-\psi\right) \\
& K_{26}=\sum_{i=1}^{N}\left(\frac{\partial p_{x}^{(i)}}{\partial \psi} K_{21}+\frac{\partial p_{y}^{(i)}}{\partial \psi} K_{22}\right)= \\
& -l^{(i)} \sin \left(\psi+\beta^{(i)}\right) K_{21}+l^{(i)} \cos \left(\psi+\beta^{(i)}\right) K_{22}= \\
& -l^{(i)} \sin \left(\psi+\beta^{(i)}\right)\left(k_{H H}^{(i)}-\bar{k}_{H H}^{(i)}\right) \cos \alpha^{(i)} \sin ^{(i)}+ \\
& l^{(i)} \cos \left(\psi+\beta^{(i)}\right)\left(k_{H H}^{(i)} \cos ^{2} \alpha^{(i)}+\bar{k}_{H H}^{(i)} \sin ^{2} \alpha^{(i)}\right)= \\
& k_{H H} l^{(i)} \sin \alpha^{(i)} \sin \left(\alpha^{(i)}-\beta^{(i)}-\psi\right)+\bar{k}_{H H} l^{(i)} \cos \alpha^{(i)} \cos \left(\alpha^{(i)}-\beta^{(i)}-\psi\right)
\end{aligned}
$$

and finally:

$$
\begin{aligned}
& K_{66}=\sum_{i=1}^{N}\left[\left(\frac{\partial p_{x}^{(i)}}{\partial \psi}\right)^{2} K_{11}+2 \frac{\partial p_{x}^{(i)}}{\partial \psi} \frac{\partial p_{y}^{(i)}}{\partial \psi} K_{12}+\left(\frac{\partial p_{x}^{(i)}}{\partial \psi}\right)^{2} K_{22}\right] \\
& -\frac{\partial^{2} p_{x}^{(i)}}{\partial \psi^{2}} F_{H} \cos \alpha^{(i)}-\frac{\partial^{2} p_{y}^{(i)}}{\partial \psi^{2}} F_{H} \sin \alpha^{(i)}= \\
& \left(-l^{(i)} \sin \left(\psi+\beta^{(i)}\right)\right)^{2} K_{11}+2\left(-l^{(i)} \sin \left(\psi+\beta^{(i)}\right) l^{(i)} \cos \left(\psi+\beta^{(i)}\right)\right) K_{12}+ \\
& \left(l^{(i)} \cos \left(\psi+\beta^{(i)}\right)\right)^{2} K_{22}+l^{(i)} \cos \left(\psi+\beta^{(i)}\right) F_{H} \cos \alpha^{(i)}+l^{(i)} \sin \left(\psi+\beta^{(i)}\right) F_{H} \sin \alpha^{(i)}= \\
& k_{H H} l^{(i)^{2}} \sin ^{2}\left(\alpha^{(i)}-\beta^{(i)}-\psi\right)+\bar{k}_{H H} l^{(i)^{2}}\left(\cos ^{2}\left(\alpha^{(i)}-\beta^{(i)}-\psi\right)+r^{(i)} / l^{(i)} \cos \left(\alpha^{(i)}-\beta^{(i)}-\psi\right)\right)
\end{aligned}
$$

As it is easy to see, the results from Equations F.11 to F.13 retrieve the coefficients presented by Pesce, Amaral and Franzini (2018). 